\title{
Investigation of immune responses contributing to the pathogenesis of load-induced heart failure and the rejection of stem cell grafts
}

\author{
Doctoral Thesis \\ In partial fulfillment of the requirements for the degree \\ “Doctor rerum naturalium (Dr. rer. nat.)" \\ in the Molecular Medicine Study Program \\ at the Georg-August University Göttingen
}

\author{
submitted by \\ Carina Hamann \\ born in
}

Hildesheim, Germany

Göttingen, June 2016 


\section{Members of the thesis committee}

Supervisor and first member of the thesis committee:

Prof. Dr. med. Ralf Dressel

Department of Cellular and Molecular Immunology

University Medical Center

Georg-August University Göttingen

Second member of the thesis committee:

Prof. Dr. rer. nat. Lutz Walter

Department of Primate Genetics

German Primate Center

Third member of the thesis committee:

Prof. Dr. rer. nat. Susanne Lutz

Department of Pharmacology

University Medical Center Göttingen

Date of Disputation: 


\section{AFFIDAVIT}

Here I declare that my doctoral thesis entitled

"Investigation of immune responses contributing to the pathogenesis of load-induced heart failure and the rejection of stem cell grafts"

has been written independently with no other sources and aids than quoted. 


\section{Table of Content}

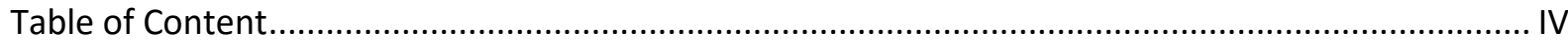

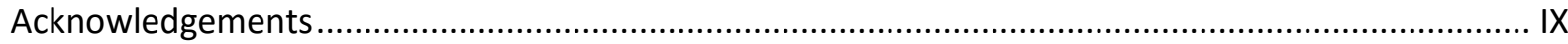

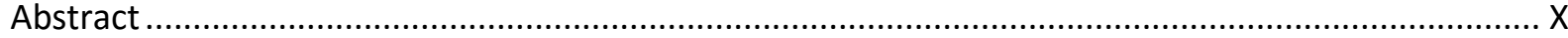

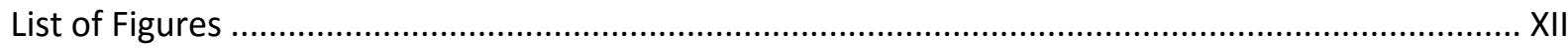

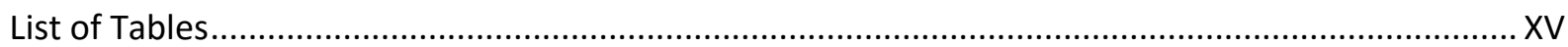

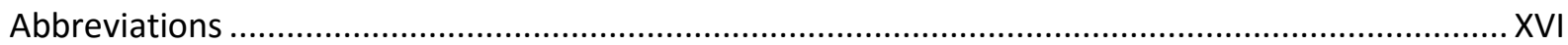

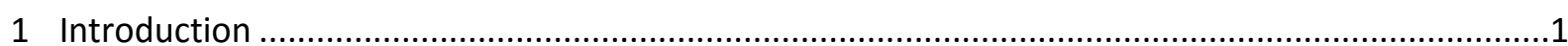

1.1 Heart failure - a disease of considerable clinical and socioeconomic relevance ..........................1

1.1.1 Therapy and molecular mechanisms of heart failure in humans .........................................2

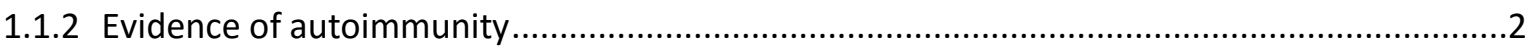

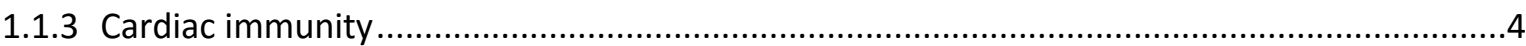

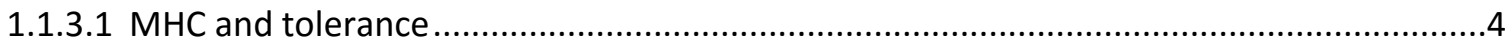

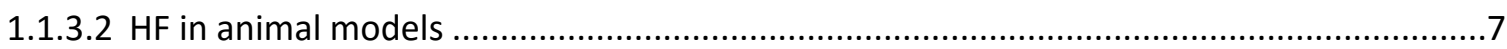

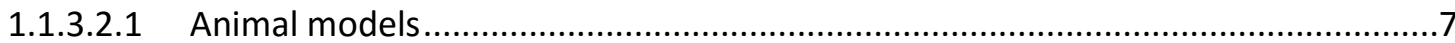

1.1.3.2.2 Murine overload models to induce HF............................................................

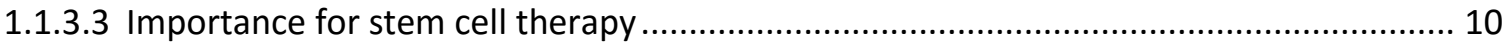

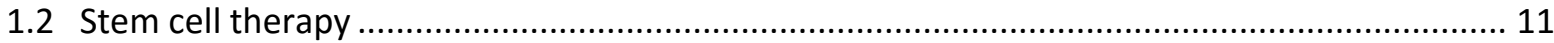

1.2.1 Stem cell types and their role for transplantation therapy .............................................. 11

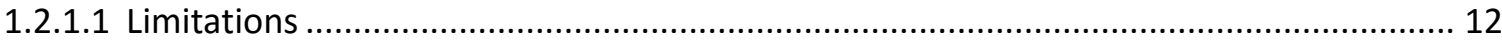

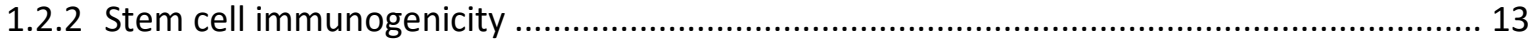

1.2.2.1 Expression of histocompatibility antigens ................................................................. 13

1.2.2.2 Susceptibility to T and NK cell killing …..................................................................... 15

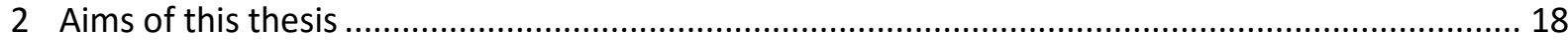

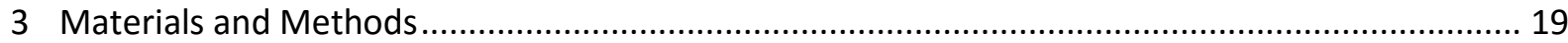

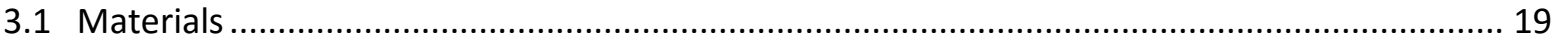

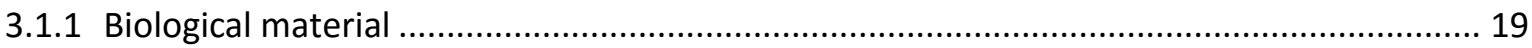

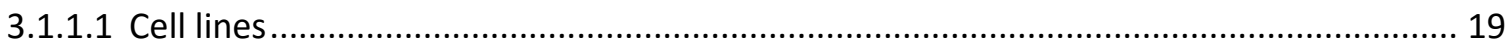

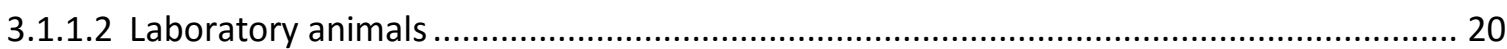

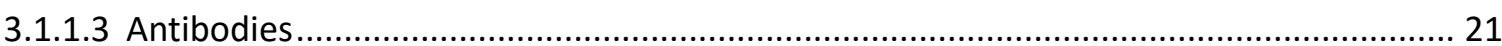

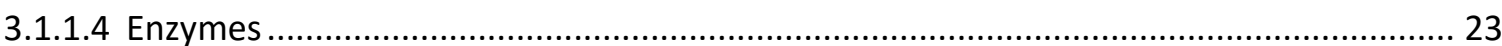

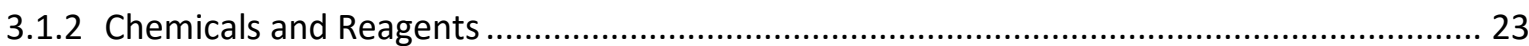

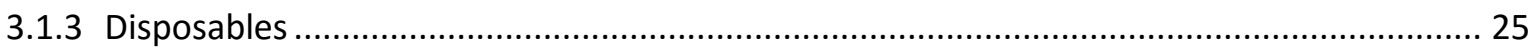

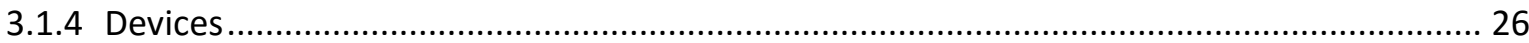




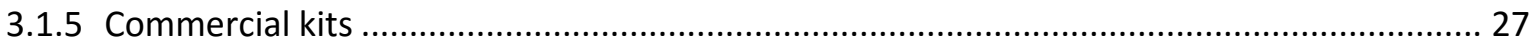

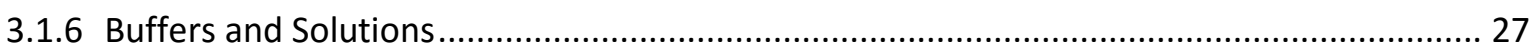

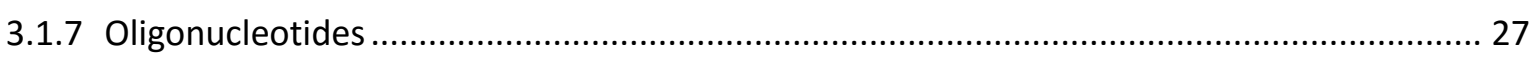

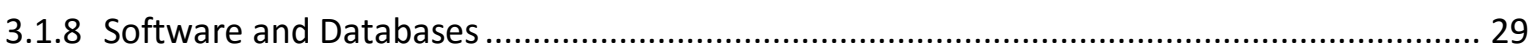

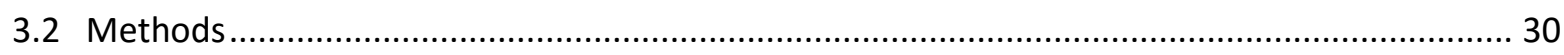

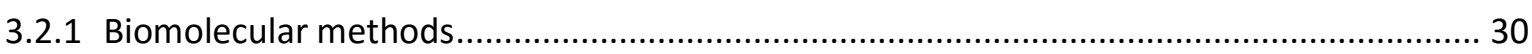

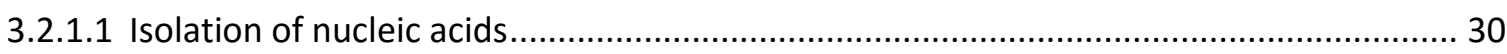

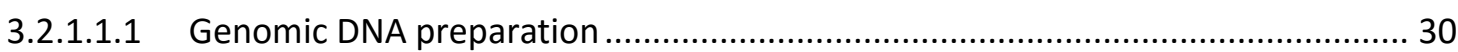

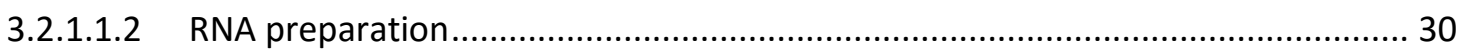

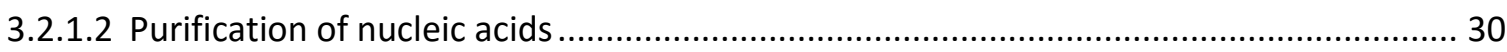

3.2.1.2.1 Purification of nucleic acids via alcohol precipitation ........................................ 30

3.2.1.2.2 Precipitation of nucleic acids via phenol-chloroform extraction .......................... 31

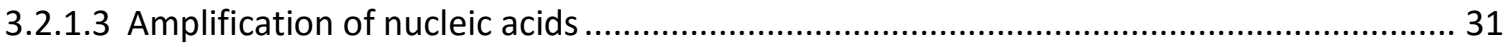

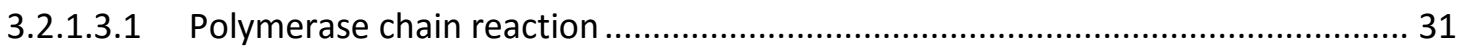

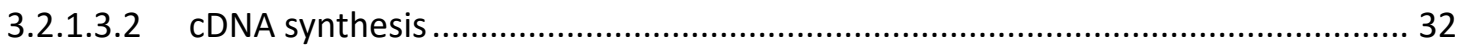

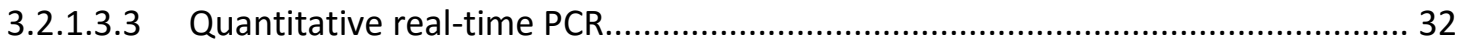

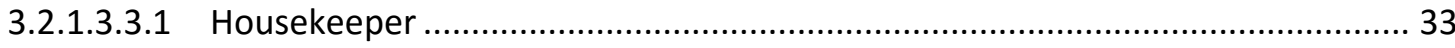

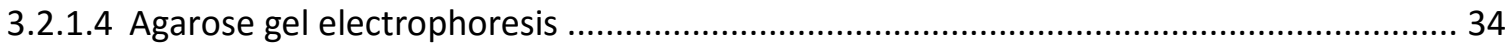

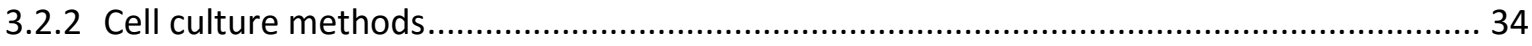

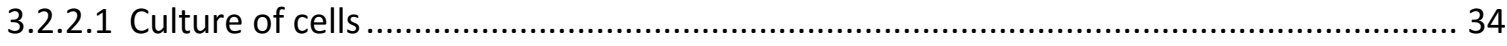

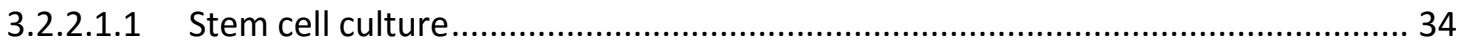

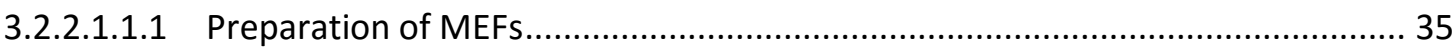

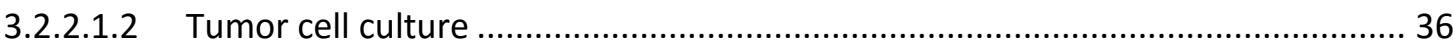

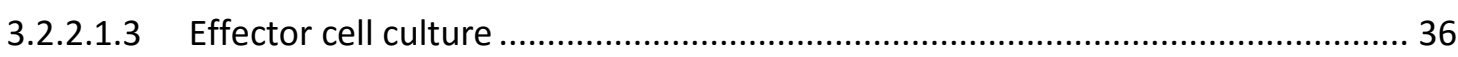

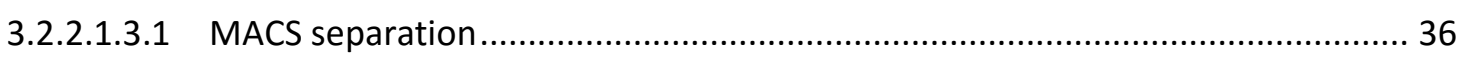

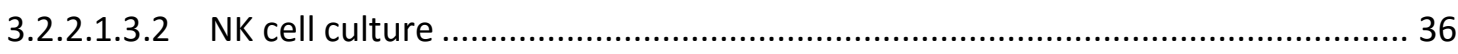

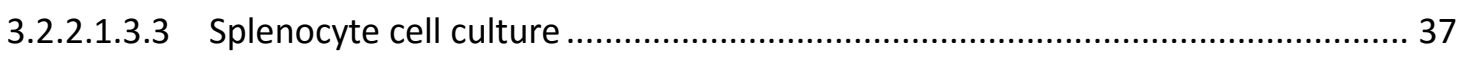

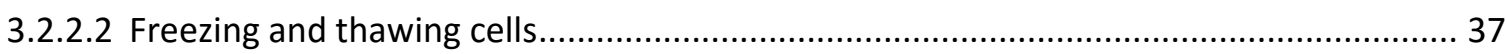

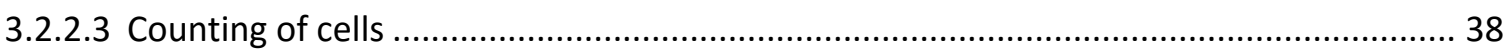

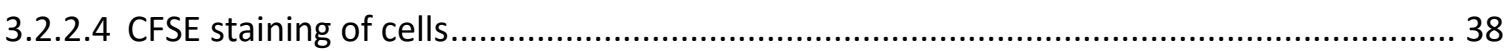

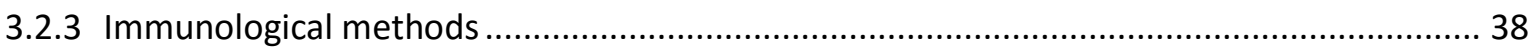

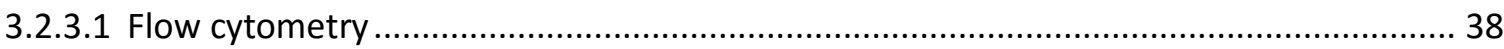

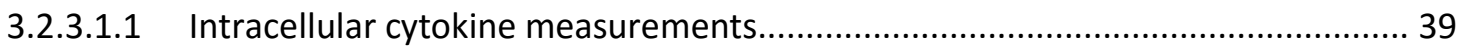

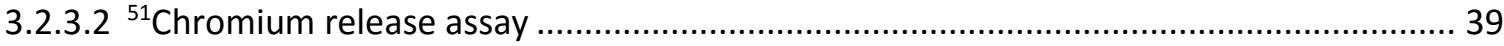

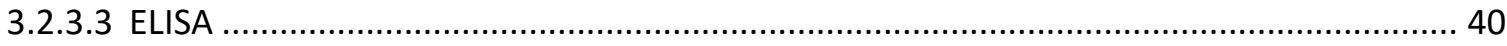




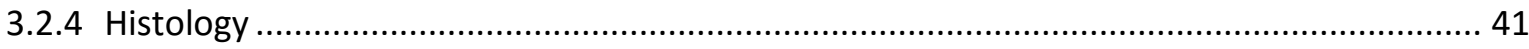

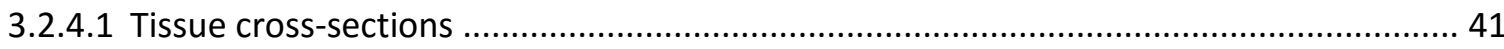

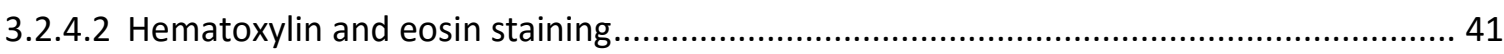

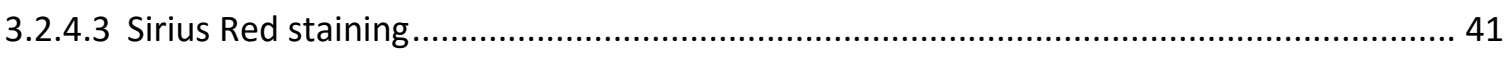

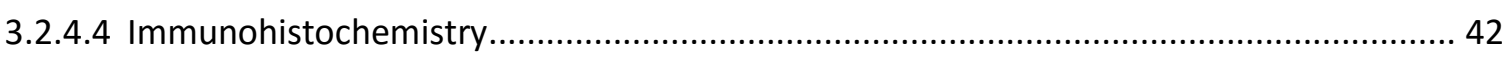

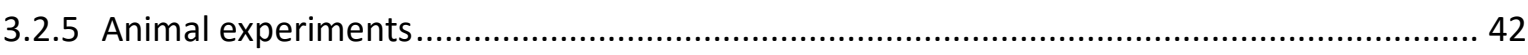

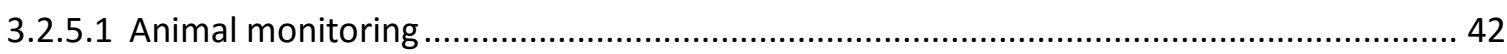

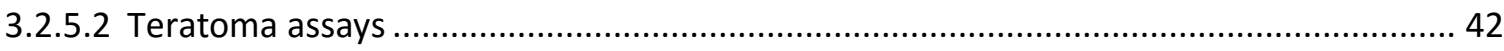

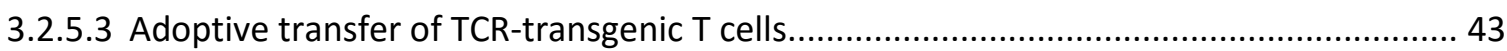

3.2.5.4 Transverse aortic constriction and echocardiography .................................................... 43

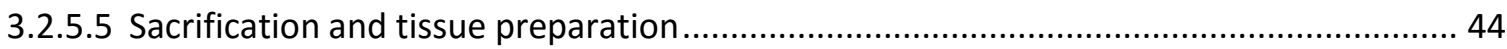

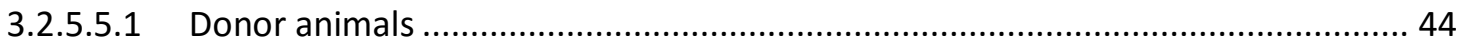

3.2.5.5.1.1 Preparation of Concanavalin A supernatant from rat spleens............................ 44

3.2.5.5.1.2 TCR-transgenic T cells from OT-I or OT-II mice .................................................... 44

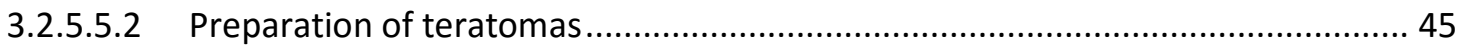

3.2.5.5.2.1 Teratoma digestion for flow cytometric analysis............................................... 45

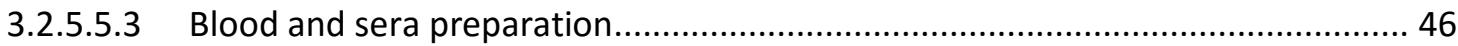

3.2.5.5.4 Preparation of TAC- and sham-operated animals................................................ 46

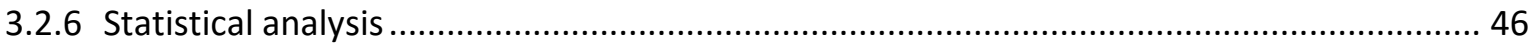

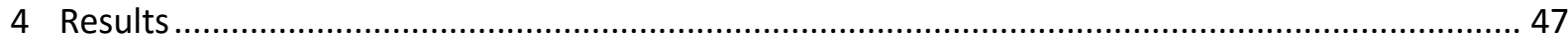

4.1 Investigation of immune response contributing to the pathogenesis of load-induced heart

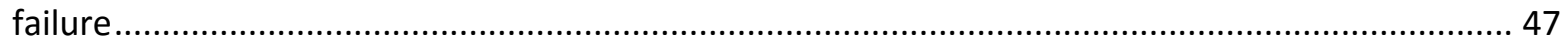

4.1.1 Adoptive transfer of TCR-transgenic T cells into cMy-mOVA mice …................................ 48

4.1.1.1 Immunohistochemical analysis of leukocyte infiltration into the myocardium ............... 50

4.1.1.2 Activation of OVA-specific cytotoxic T lymphocytes .................................................... 53

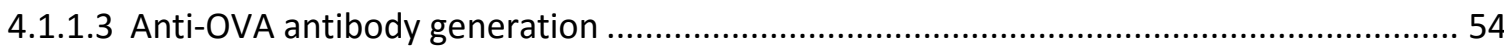

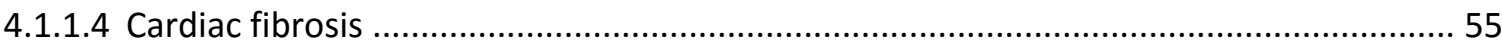

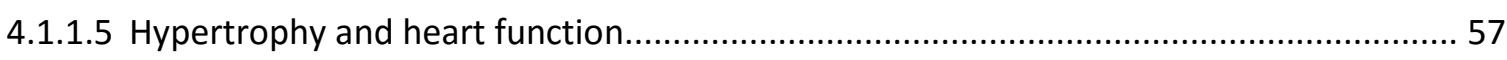

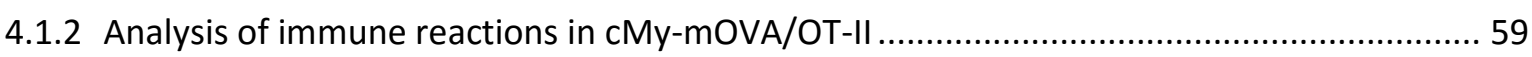

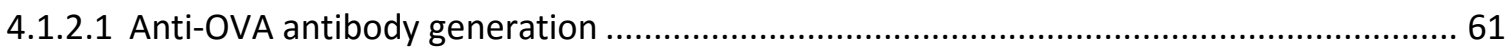

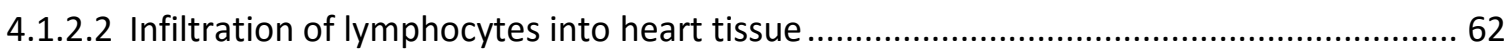

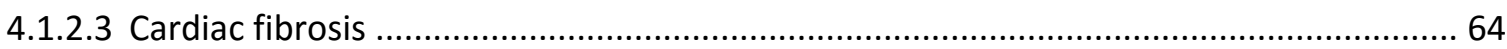

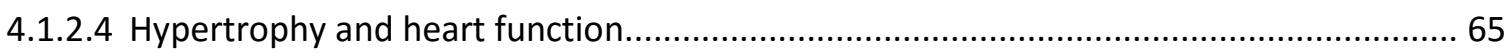

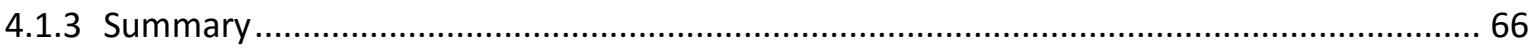

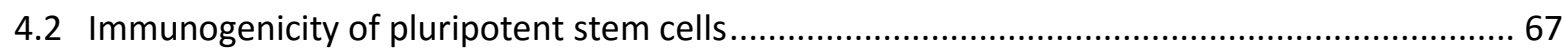


4.2.1 Tumor formation in immunodeficient hosts

4.2.1.1 Effects of OVA expression on tumor formation, size and weight in immunodeficient hosts.

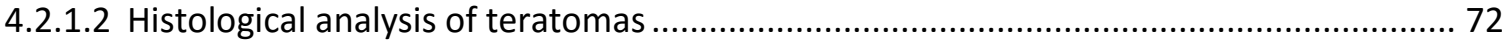

4.2.2 Adoptive transfer of OVA-specific T cells into immunodeficient hosts............................... 73

4.2.2.1 Effect of TCR-transgenic T cells on tumor formation .................................................... 75

4.2.3 Effect of OVA expression in stem cells on tumor formation in immunocompetent hosts ... 78

4.2.3.1.1 Effect of OVA expression in stem cells on various parameters in

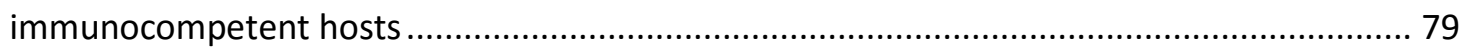

4.2.4 Comparative analysis of tumor formation and characteristics in immunodeficient and

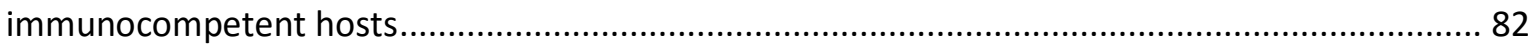

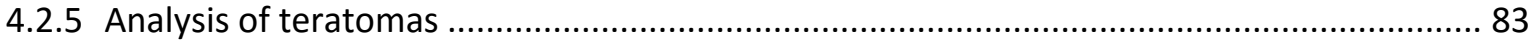

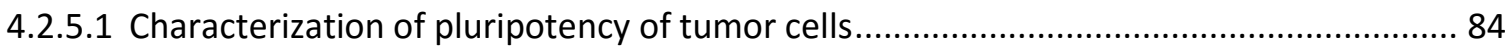

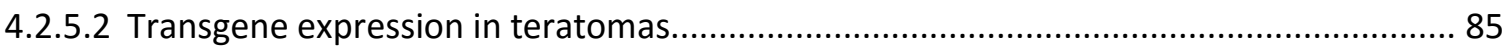

4.2.5.3 Expression of amino acid-depleting enzymes in teratomas ......................................... 86

4.2.5.4 Expression of immunogenicity-causing genes ........................................................... 88

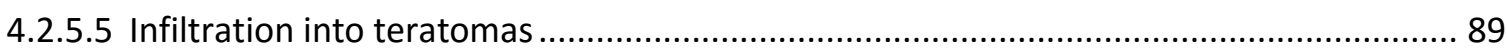

4.2.6 Generation of OVA-specific CTLs in syngeneic hosts ................................................... 92

4.2.7 Killing of wildtype and OVA-expressing iPSCs and ESCs by NK cells ................................. 98

4.2.8 OVA-specific antibody generation in syngeneic hosts.................................................. 99

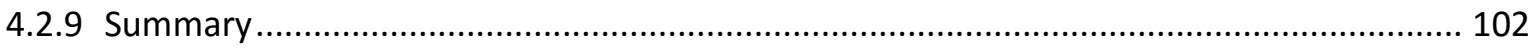

4.3 The role of NKG2D in the killing of pluripotent stem cells ............................................... 103

4.3.1 Killing of murine stem cells by naïve C57BL/6 and NKG2D-deficient NK cells .................. 103

4.3.2 Killing of murine stem cells by IL-2-activated wildtype C57BL/6 and NKG2D-deficient NK

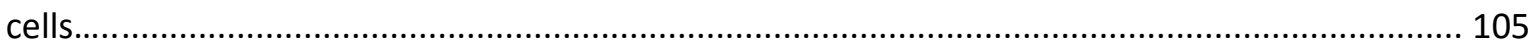

4.3.3 Expression of ligands of the NKG2D receptor on murine stem cells ............................... 107

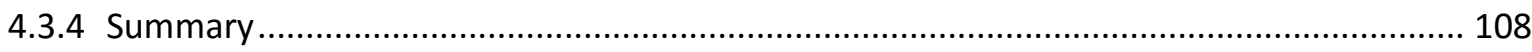

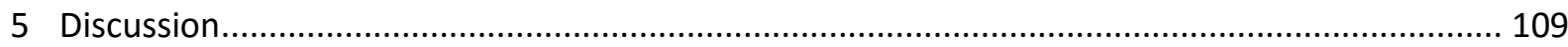

5.1 Involvement of autoimmune responses on the pathogenesis of load-induced heart failure.. 109

5.1.1 Increased leukocyte infiltration into the myocard after TAC ........................................ 109

5.1.1.1 Controversial influence of endothelial surface adhesion molecules on immune cell

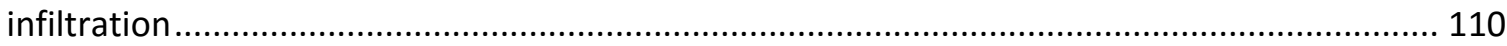

5.1.2 Activation of adaptive immune responses towards cardiac antigens .............................. 110

5.1.3 The role of adaptive immune responses in the progression to heart failure ..................... 112

5.1.4 Increased numbers of autoreactive $T$ cells accelerate progression of load-induced heart

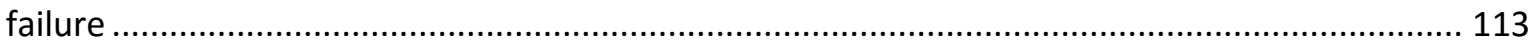

5.1.5 Immune mechanisms contributing to heart failure...................................................... 114 


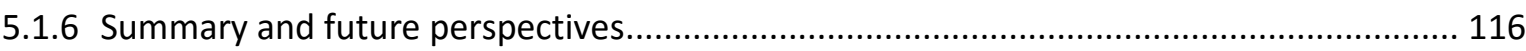

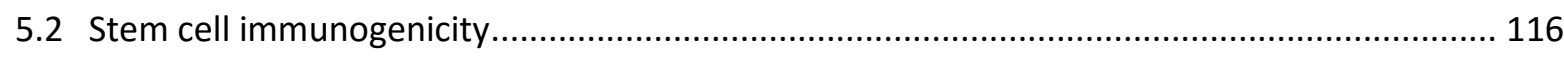

5.2.1 Influence of minor histocompatibility antigen expression on stem cell immunity ............ 117

5.2.1.1 Expression of OVA as miHC antigen affects engraftment of stem cells in

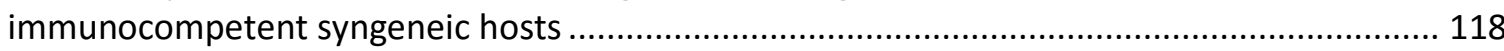

5.2.1.2 Expression of a miHC antigen led to OVA-specific cytotoxic T lymphocyte induction ... 119

5.2.1.3 Expression of a miHC antigen resulted in OVA-specific antibody generation and $\mathrm{CD}^{+} \mathrm{T}$

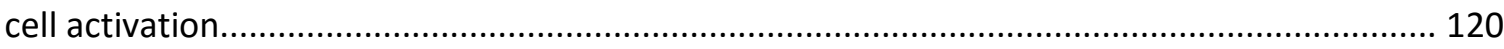

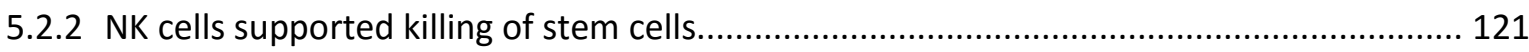

5.2.3 Analysis of mechanisms potentially influencing the immunogenicity of stem cell and stem cell-derived grafts 122

5.2.3.1 Negligible expression of immunogenic genes in stem cells and their derivates........... 122

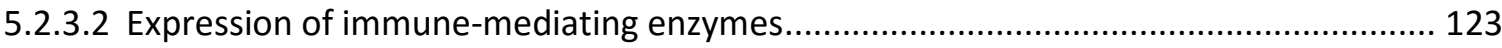

5.2.4 MiHC antigen expression affects engraftment by inducing an antigen dependent immune

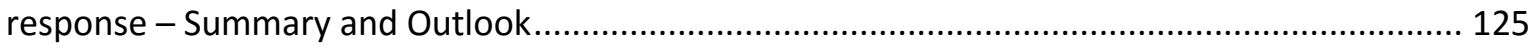

5.3 Killing of murine pluripotent stem cells largely depends on the activating NK receptor

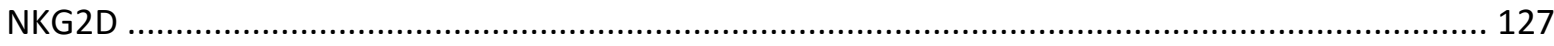

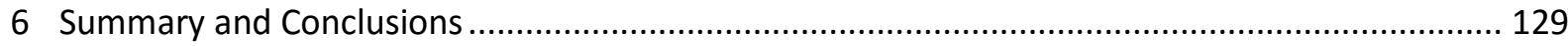

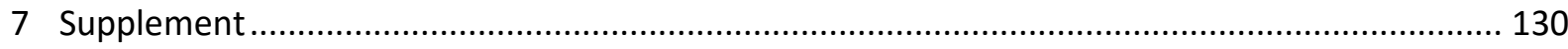

7.1 Investigation of immune response contributing to the pathogenesis of load-induced heart

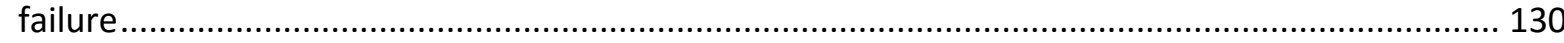

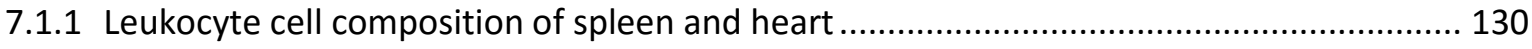

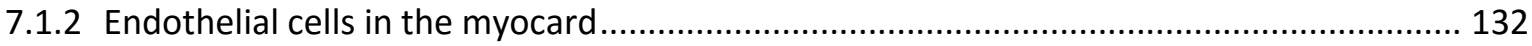

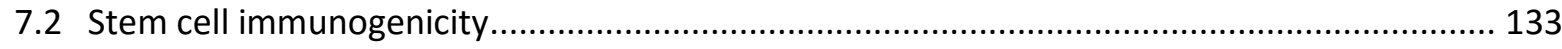

7.2.1 Germ layer marker expression in tumors derived from wildtype and OVA-expressing stem cells in immunodeficient and immunocompetent host......................................................... 133

7.2.2 Influence of adoptive transfer on tumor characteristics in $\mathrm{RAG}^{-{ }^{-}} \mathrm{\gamma c}^{-{ }^{-}}$mice .................... 135

7.2.3 Influence of $\mathrm{HY}$ antigens on tumor formation in immunocompetent mice ....................... 136

7.2.4 Leukocyte infiltration into teratomas analyzed by flow cytometry ............................... 137

7.2.5 Cellular composition of lymphocytes of host splenocytes after stem cell injections ......... 138

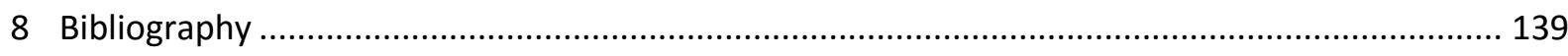

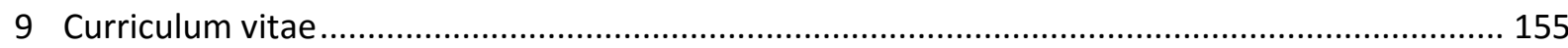




\section{Acknowledgements}

Hiermit möchte ich mich zuallererst bei meinem Betreuer und ersten Prüfer Prof. Dr. Ralf Dressel, für die Bereitstellung meines Themas, Mittel und stetige Unterstützung bedanken. Als Ansprechpartner bei auftretenden Problemen war er stets für mich da. Des Weiteren möchte ich mich bei meinen Thesis Committee Mitgliedern Prof. Dr. Lutz Walter und Prof. Dr. Susanne Lutz bedanken, die stets für Diskussionen und Ratschläge offen waren. Herzlichen Dank auch an das ganze Institut und besonders Prof. Dr. Jürgen Wienands, für die herzliche Arbeitsatmosphäre, und Bereitstellung unserer Laborräume.

Meinen Laborkollegen gilt besonderen Dank, da sie mir stets mit Rat und Tat zur Seite standen. Insbesondere Leslie Elsner sorgte oft für reibungslose Abläufe durch ihr besonderes Multitasking und Überblick über die gesamte Einrichtung. Zusätzlich verdient mein Vorgänger und Hin-und-wieder Kollege Dr. Sebastian Monecke einen großen Dank; dafür, dass er mich in die komplexe Welt der Stammzellkultur und in die immunologischen Methoden eingearbeitet hat, aber auch für die lustigen Momente in unserem Büro. Hier darf natürlich Pranali Shah nicht unerwähnt bleiben, die als Kollegin bei uns ihre Doktorarbeit begann, Sie jedoch als gute Freundin beenden wird. Danke Dir und Kanika Vanshylla für eure Hindi-Lernstunden und eurer stets offenes Ohr für all meine Launen und Probleme. Natürlich möchte ich auch allen anderen Kollegen danken, dessen Erwähnung hier allerdings den Rahmen sprengen würde: Danke an Euch für Snacks, Kaffee, Klatsch und Tratsch, gute Laune und Bereitstellung von eventuell fehlenden Arbeitsmaterialien. Dank auch an meine außerinstitutionellen Freunde für Ratschläge und Weisheiten über wissenschaftliches Arbeiten, die gerne auch am späteren Abend besprochen worden sind.

Großer Dank gilt auch meiner Familie, besonders meinen Eltern Jürgen und Rosemarie Hamann, für jegliche Unterstützung, Belehrung, Motivation und die letzten 28 Jahre. Meinen Brüdern Christian und Michael möchte ich für die Herausforderungen die ihr mir in jungen Jahren gebracht habt bedanken, dies hat mich gut auf die Doktorarbeit vorbereitet. Außerdem einen goldigen Dank an Amy und Collin, die mir stets nach einen langen Arbeitstag den Abend versüßt haben. Dies gilt auch für Schrödinger und Murphy, meine beiden Rabauken zuhause, die mir gerade während des Schreibprozesses unterstützend zur Seite standen. Da das Beste zum Schluss kommt: Den größten, herzlichsten und liebevollsten Dank an Gero, der mich jederzeit in jeglicher Hinsicht unterstützt hat. Dabei hat er mich immer wieder motiviert, unterstützt, bejubelt, ermahnt, aufgeheitert, unterhalten, und einfach so genommen, wie ich bin. Ich danke dir dafür - Ich liebe dich! 


\section{Abstract}

Heart failure (HF) is often associated with inflammation and activation of the immune system. Moreover, the development to HF has been shown to be accompanied by autoimmune reactions since cardiac autoantibodies were identified in many studies. However, the role of T cells in load-induced HF had not been addressed previously. We hypothesized that an aortic constriction leading to hypertrophy and subsequently to HF can activate autoreactive T cells, which contribute to the progression of the disease. To examine autoimmune responses towards a cardiomyocyte-specific antigen, cMy-mOVA mice were used, which express ovalbumin (OVA) selectively on cardiomyocytes. In these mice, HF was induced by increasing the afterload by transverse aortic constriction (TAC). It was previously demonstrated in our group that cMy-mOVA mice did not show an accelerated impairment of heart function after TAC operation, although an activation of OVA-specific cytotoxic T lymphocytes (CTLS) was observed at a low level. To clarify the role of autoreactive T cells, TACoperated cMy-mOVA mice were challenged by transfer of OVA-specific $\mathrm{CD}^{+}$and $\mathrm{CD} 8^{+} \mathrm{T}$ cells. The adoptive transfer of $\mathrm{CD}^{+}$and/or $\mathrm{CD}^{+}$did not significantly accelerate progression to HF. However, double-transgenic cMy-mOVA/OT-II mice, in which the majority of T cells are OVA-specific CD4 ${ }^{+} \mathrm{T}$ cells, showed an accelerated progression towards HF. Since these mice did not develop OVA-specific autoantibodies, we could demonstrate that $\mathrm{CD} 4^{+} \mathrm{T}$ cells with specificity for a cardiomyocyte-specific autoantigen can promote the progression from hypertrophy to HF independent of autoantibodies.

The immunological milieu within the myocard might not only affect the progression of HF but also the chances of repair by new regenerative therapies, e.g. the transplantation of stem cell-derived grafts. To clarify the probability of engraftment and reduce the risk of complications for the recipient after stem cell-derived graft transplantations, the immunogenicity of pluripotent stem cells (PSCs) and their differentiation products has to be considered. It is known that the mismatch of major histocompatibility complex (MHC) molecules lead to acute rejection of grafts but additionally, minor histocompatibility (miHC) antigens affect engraftment of organs even between MHC-matched individuals. Consequently, the role of miHC antigens in PSCs and their derivates need to be clarified since they cannot be matched in allogeneic transplantations. For this, OVA-expressing embryonic stem cells (ESCs) and induced pluripotent stem cells (iPSCs) were used to monitor immune responses against OVA as a model of a miHC antigen after injection into otherwise syngeneic mice. It was demonstrated that the expression of OVA led to a significantly reduced rate of engraftment of PSCs. OVA-specific CTLs and OVA-specific antibodies were observed in the hosts. Therefore, a single antigen 
can be sufficient to induce rejection of transplanted PSC-derived grafts suggesting that therapies using grafts derived from MHC-matched allogeneic PSCs will likely require immunosuppressive or immunomodulatory treatment.

Since PSCs are at risk to give rise to teratomas if residuals of pluripotent cells remain in a graft after in vitro differentiation, the susceptibility of PSCs to natural killer (NK) cells was analyzed. Previous antibody inhibition studies indicated that the killing of murine PSCs by NK cells was mainly mediated by the activating NK cell receptor NKG2D. To clarify the role of NKG2D for killing of murine PSCs, NKG2D-deficient and wildtype NK cells were analyzed concerning their potential to kill several PSC lines including multipotent adult germline stem cells (maGSCs), ESCs and iPSCs. Naïve NKG2D-deficient NK cells failed to kill PSCs. Stimulation of NK cells by interleukin-2 (IL-2) increased the killing but could not completely compensate the NKG2D deficiency. Hence, it was demonstrated that NKG2D is an important activating receptor involved in killing of murine PSCs. 


\section{List of Figures}

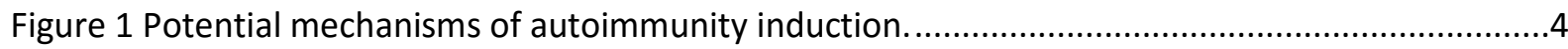

Figure 2 Tolerance induction of T lymphocytes in the thymus and periphery. ..................................6

Figure 3 Exemplary pictures of sham- and TAC-operated hearts of C57BL/ 6 mice ............................8

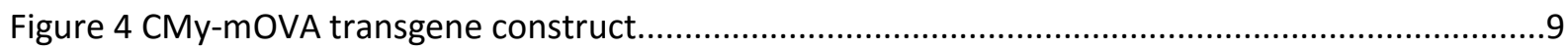

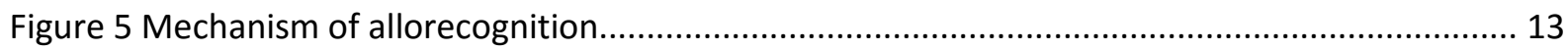

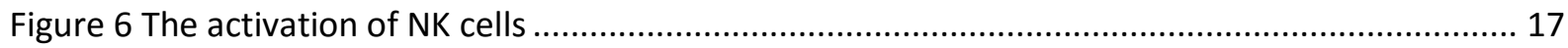

Figure 7 Flow cytometric analysis of transferred T cells in blood samples....................................... 49

Figure 8 Flow cytometric analysis of transferred T cells in spleens................................................ 50

Figure $9 \mathrm{IHC}$ analysis of lymphocyte infiltration into myocardia of cMy-mOVA mice ......................... 51

Figure 10 Immunohistochemical analysis of the myocardium ...................................................... 52

Figure 11 Cytotoxicity assays performed with splenocytes.......................................................... 54

Figure 12 Anti-OVA-antibody titer in cMy-mOVA mice .................................................................. 55

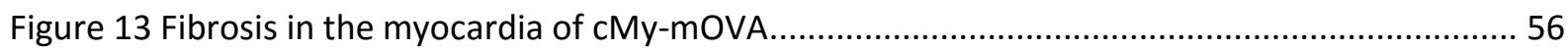

Figure 14 Heart weight of cMy-mOVA mice and mice which received OVA-specific T cells. .............. 57

Figure 15 Analysis of hypertrophy and heart function of cMy-mOVA mice by echocardiography...... 58

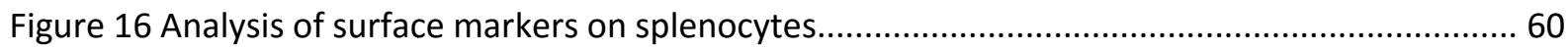

Figure 17 Proportion of $\mathrm{CD}^{+}{ }^{+} \mathrm{T}$ cells of cMy-mOVA/OT-II mice expressing intracellular cytokines. .... 61

Figure 18 Anti-OVA antibody titers in sera of cMy-mOVA/OT-II mice ............................................. 62

Figure $19 \mathrm{IHC}$ analysis of infiltrating cells into the myocardia of cMy-mOVA/OT-II animals................ 63

Figure 20 Fibrosis in the myocardia of cMy-mOVA/OT-II mice after TAC and sham operations.......... 64

Figure 21 Hypertrophy of TAC- and sham-operated cMy-mOVA and cMy-mOVA/OT-II.................... 65

Figure 22 Analysis of functional parameters assessing heart function of cMy-mOVA/OT-II............... 66

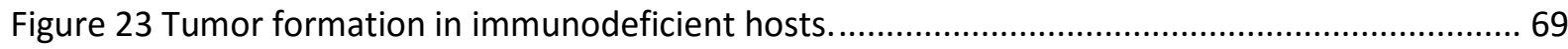

Figure 24 Analysis of $(A)$ tumor formation, $(B$, tumor size and $(C)$ tumor weight ............................. 70

Figure 25 Histological example pictures of teratomas formed after injection of iPSC 129/Sv............ 72

Figure 26 Schematic overview over adoptive transfer experiments .............................................. 73

Figure 27 Flow cytometric blood analysis of transferred T cells of RAG2 ${ }^{-1-\gamma c^{-1-}}$ mice ......................... 74

Figure 28 Tumor formation in immunodeficient hosts................................................................ 75

Figure 29 Histological analysis of T cell infiltration into teratomas. ................................................ 76

Figure 30 Analysis of OVA-specific CTL activation examined via ${ }^{51} \mathrm{Cr}$ release assays. ........................ 77

Figure 31 Kaplan-Meier survival curve of immunodeficient animals ............................................. 77 
Figure 32 Tumor formation in immunocompetent 129/Sv mice................................................. 78

Figure 33 Tumor frequency after ESC injections into immunocompetent 129/Sv host..................... 79

Figure 34 Analysis of $(A)$ tumor formation, (B) tumor size and (C) tumor weight ............................. 80

Figure 35 Percentage of tumor formation and rejection in male and female mice ........................... 82

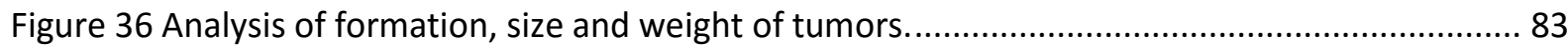

Figure 37 QPCR analysis of pluripotency and transgene markers in teratomas................................ 84

Figure 38 Analysis of cell proliferation and pluripotency via IHC stainings of teratoma tissue........... 85

Figure 39 QPCR analysis of transgene markers in teratomas. ......................................................... 86

Figure 40 QPCR analysis of $I d o$ and $A r g 1$ gene expression in teratomas.......................................... 87

Figure 41 QPCR analysis of 'immunogenicity-causing' gene expression in teratomas....................... 89

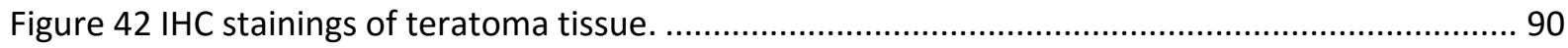

Figure 43 Analysis of leukocyte infiltration into teratomas analyzed by IHC. ................................. 91

Figure 44 Flow cytometric analysis of restimulated splenocytes .................................................. 92

Figure 45 Analysis of OVA-specific CTLs in 129/Sv animals which received stem cell inoculations..... 93

Figure 46 Analysis of the presence of OVA-specific T cells in syngeneic hosts (long-term)............... 94

Figure 47 Analysis of OVA-specific CTL responses ...................................................................... 95

Figure 48 Analysis of the presence of OVA-specific T cells in syngeneic 129/Sv animals................... 96

Figure 49 Analysis of the presence of OVA-specific T cells after stem cell inoculation...................... 97

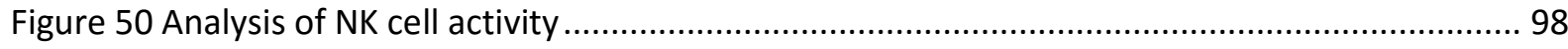

Figure 51 Analysis of OVA-specific antibody titers in syngeneic 129/Sv mice............................... 100

Figure 52 Analysis of OVA antibody isotype distribution in sera of 129/Sv mice ........................... 101

Figure 53 Analysis of OVA-specific antibody titer in syngeneic hosts (1 week).............................. 102

Figure 54 Killing of different stem cell lines and control YAC-1 cells by NK cells............................. 104

Figure 55 Killing of various stem cells lines by naïve NK cells....................................................... 105

Figure 56 Killing of various cell lines by IL-2 stimulated NK cells.................................................. 106

Figure 57 Killing of various stem cells lines by in vitro IL-2 stimulated NK cells. ............................. 107

Figure 58 Expression of NKG2D ligands on stem cell lines and control (YAC-1) cell........................ 108

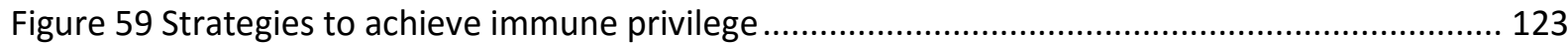

Figure 60 Proportion of lymphocytes and myeloid cells in the (A) spleen and (B) myocardium........ 131

Figure 61 Flow cytometric analysis of intercellular adhesion molecules ICAM-1 and VCAM-1 ........ 132

Figure 62 Germ layer marker expression in teratomas. ............................................................. 134

Figure 63 Analysis of $(\mathrm{A})$ tumor size and (B) tumor weight. ...................................................... 135 
Figure 64 Tumor formation after injection of OVA-expressing stem cells...................................... 136

Figure 65 Analysis of leukocyte infiltration into teratomas analyzed by flow cytometry. ................ 137

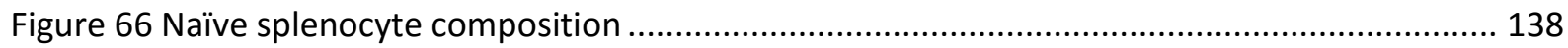




\section{List of Tables}

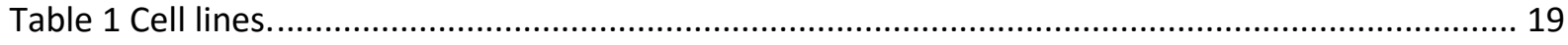

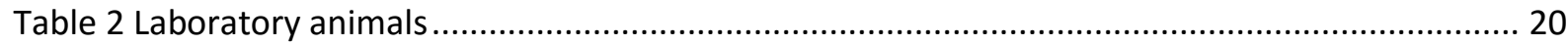

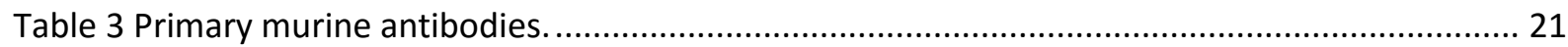

Table 4 Secondary antibodies and ELISA antibodies.................................................................. 22

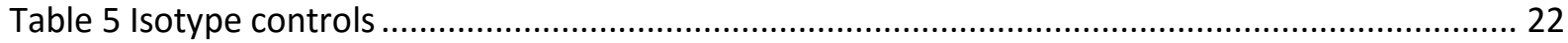

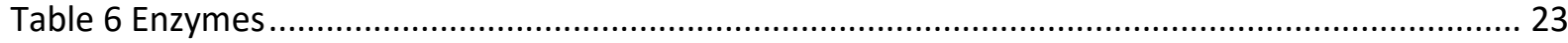

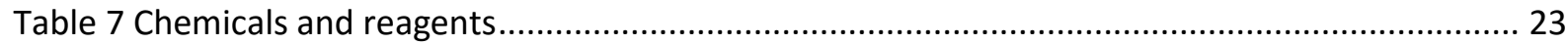

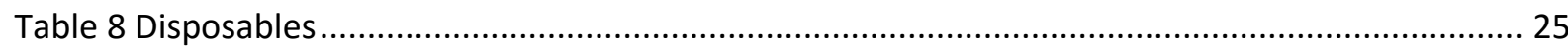

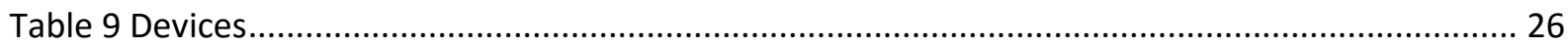

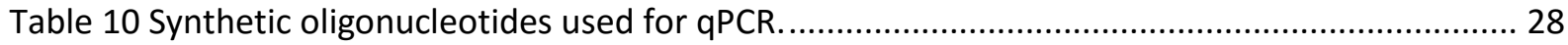

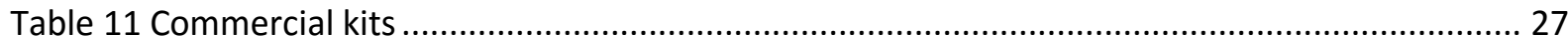

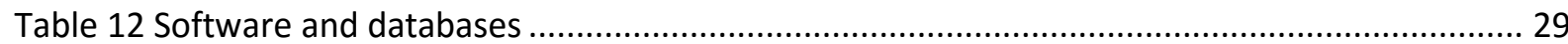

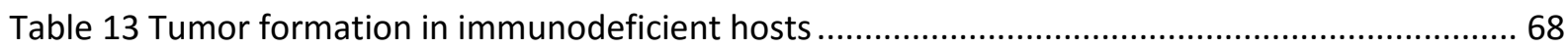

Table 14 Analysis of tumor formation in immunodeficient mice …................................................. 71

Table 15 Analysis of tumor formation in immunocompetent mice ................................................ 81 


\section{Abbreviations}

\section{-}

$\#$

$\%$

$-$

$+$

$x$

${ }^{\circ} \mathrm{C}$

I

II

$\alpha$

$\alpha-\mathrm{MHC}$

$\beta$

$\delta$

$\Delta$

$\gamma$

$\mu$

$\mu \mathrm{Ci}$

A

A

aa

ABTS

Afp

Ag

Alb

APC

Arg1

BBB

BCR

BD

BGHpA

bidest.

BSA

$C$

CAD

CD

CDNA

CFSE

Ch.

CNVs

Con A

confl.

cpm

ct

CTL

Ctnt

Cyp3a11

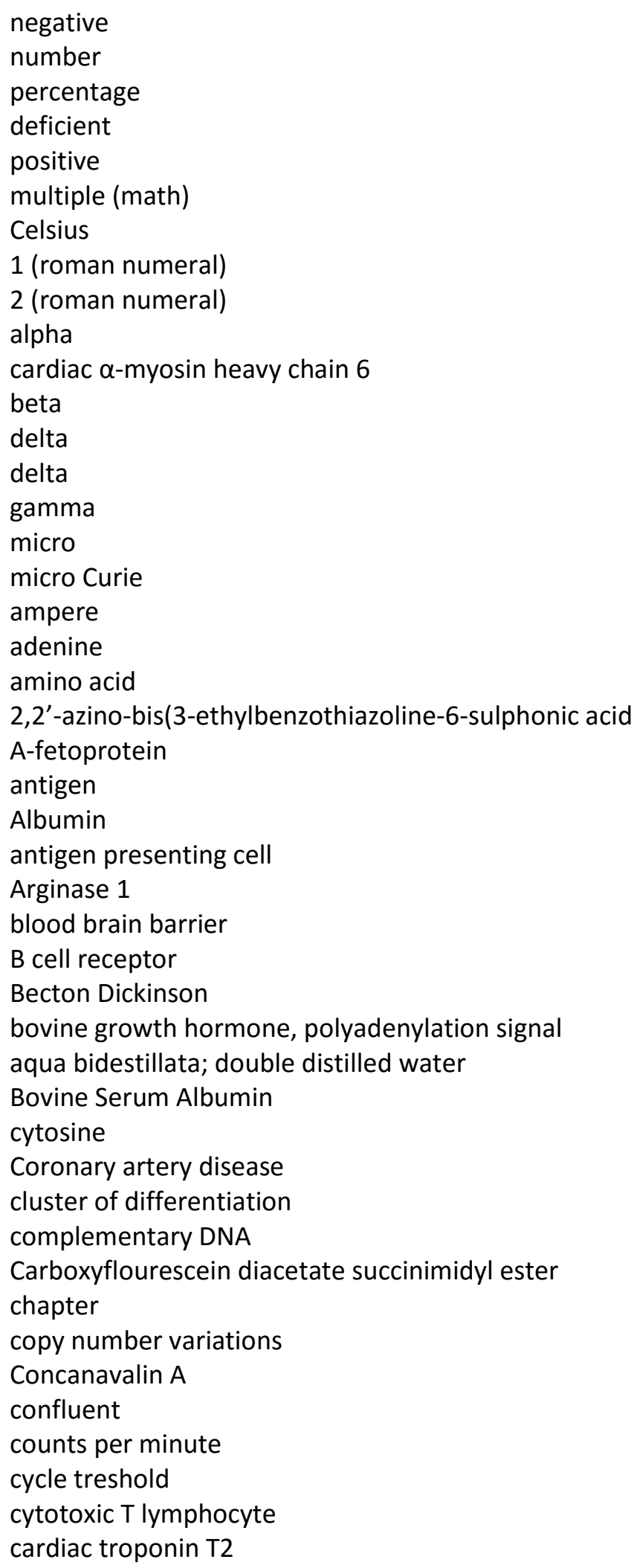




\begin{tabular}{|c|c|}
\hline $\mathrm{DAB}$ & 3,3'-diaminobenzidine \\
\hline DAMP & damage-associated molecular pattern \\
\hline DB & database \\
\hline DCM & dilated cardiomyopathy \\
\hline DCs & dendritic cells \\
\hline $\mathrm{dH}_{2} \mathrm{O}$ & distilled water \\
\hline DMEM & Dulbecco's Modified Eagle's medium \\
\hline DMSO & dimethylsulfoxide \\
\hline DNA & desoxyribonucleic acid \\
\hline dNTPs & deoxynucleotide triphosphates \\
\hline DS & Down syndrome \\
\hline DTT & DL-Dithiothreitol \\
\hline e.g. & exempli gratia; for example \\
\hline$E: T$ & effector to target \\
\hline EBs & embryoid bodies \\
\hline EDTA & Ethylenediaminetetraacetic acid \\
\hline EF & ejection fraction \\
\hline eGFP & enhanced green fluorescent protein \\
\hline ELISA & enzyme-linked immunosorbent assay \\
\hline ESC & embryonic stem cell \\
\hline EtOH & ethanol \\
\hline f & female \\
\hline FACS & fluorescence activated cell sorting \\
\hline FAS & fractional area shortening \\
\hline FasL & Fas ligand \\
\hline FCS & Fetal calf serum \\
\hline FITC & fluoresceinisothiocyanate \\
\hline frag & DNA fragment \\
\hline G & guanine \\
\hline Gapdh & Glyceraldehyde 3-phosphate dehydrogenase \\
\hline GrB & granzyme B \\
\hline GVHD & graft versus host disease \\
\hline$H \& E$ & hematoxylin and eosin \\
\hline h/hrs & hour/hours \\
\hline $\mathrm{HCl}$ & hydrochloric acid \\
\hline HD & Huntington disease \\
\hline HF & heart failure \\
\hline HLA & human leukocyte antigen \\
\hline Hormad1 & HORMA domain-containing protein1 \\
\hline Hprt & Hypoxanthine-guanine phosphoribosyltransferase \\
\hline HRP & Horseradish peroxidase \\
\hline HSCT & hematopoietic stem cell transplantation \\
\hline i.e. & id est; that is \\
\hline i.v. & intravenous \\
\hline ICAM-1 & intercellular adhesion molecule 1 (CD54) \\
\hline ICM & intracellular cytokine measurement \\
\hline ID & identity \\
\hline Ido & Indoleamine 2,3-dioxygenase \\
\hline
\end{tabular}




\begin{tabular}{|c|c|}
\hline IDT & Integrated DNA technology \\
\hline IFN & interferon \\
\hline $\lg$ & immunoglobulin \\
\hline $\mathrm{IHC}$ & immunohistochemistry \\
\hline IHD & ischemic heart disease \\
\hline IL & interleukin \\
\hline indels & base-pair insertions or deletions \\
\hline iPSC & induced pluripotent stem cell \\
\hline ISQAVHAAHAEINEAGR & OVA peptide aa 323-339 \\
\hline ITIM & immunoreceptor tyrosine-based inhibitory motif \\
\hline IVC & individually-ventilated cage \\
\hline kb & kilo base pairs \\
\hline $\mathrm{KCl}$ & potassium chloride \\
\hline $\mathrm{KH}_{2} \mathrm{PO}_{4}$ & potassium phosphate \\
\hline KIRs & killer immunoglobulin-like receptors \\
\hline $\mathrm{L}$ & ligand \\
\hline I & liter \\
\hline LIF & leukemia Inhibory Facator \\
\hline LSC & liquid scintillation counter \\
\hline LV & left ventricle/ ventricular \\
\hline LVEDD & inner diameter of the left ventricle \\
\hline $\mathrm{M}$ & molar \\
\hline $\mathrm{m}$ & male \\
\hline M & gene stability measure \\
\hline $\mathrm{mAb}$ & monoclonal antibody \\
\hline MACS & magnetic-activated cell sorting \\
\hline maGSCs & multipotent adult germline stem cells \\
\hline MEFs & mouse embryonic fibroblasts \\
\hline $\mathrm{MFI}$ & mean fluorescence intensity \\
\hline $\mathrm{MgCl}_{2}$ & magnesium chloride \\
\hline $\mathrm{MHC}$ & major histocompatibility complex \\
\hline $\mathrm{MI}$ & myocardial infarction \\
\hline $\mathrm{miHC}$ & minor histocompatibility \\
\hline $\min$ & minute(s) \\
\hline miRNA & microRNA \\
\hline $\mathrm{ml}$ & milliliter \\
\hline $\mathrm{mm}$ & millimeter \\
\hline $\mathrm{mmHg}$ & millimeters of mercury \\
\hline M-MLV & Moloney murine leukemia virus \\
\hline $\mathrm{MRI}$ & magnetic resonance imaging \\
\hline mRNA & messenger RNA \\
\hline $\mathrm{ms}$ & mouse \\
\hline MSC & mesenchymal stem cell \\
\hline MTA & maternally transmitted antigen \\
\hline MyHC & cardiac myosin heavy chain \\
\hline $\mathrm{n}$ & nano \\
\hline $\mathrm{Na}_{2}{ }^{51} \mathrm{CrO}$ & sodium chromate \\
\hline $\mathrm{NaCl}$ & sodium cloride \\
\hline
\end{tabular}




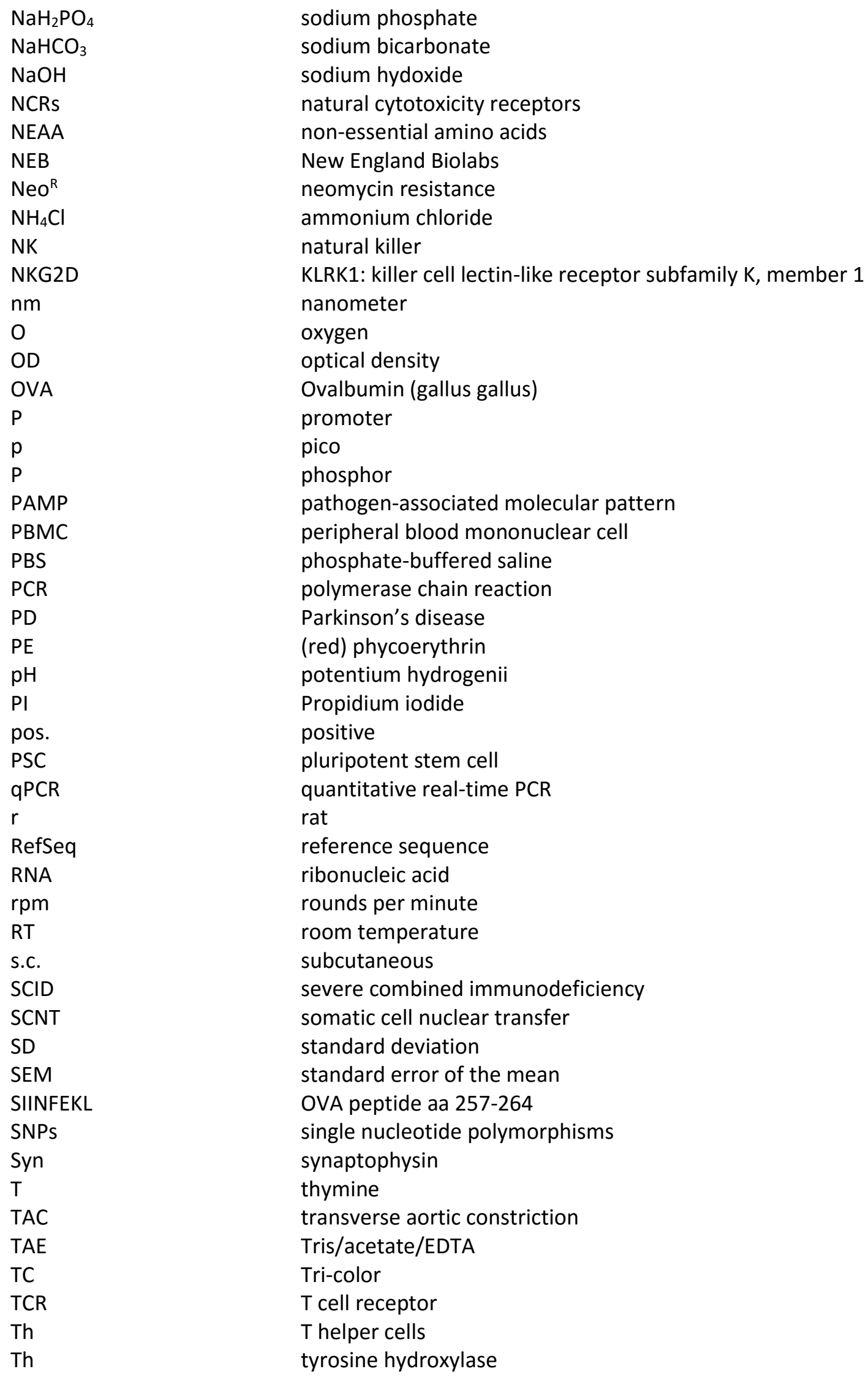




$\begin{array}{ll}\text { TLR } & \text { Toll-like receptors } \\ \text { TM } & \text { transmembrane domain } \\ \text { Tregs } & \text { regulatory T cells } \\ \text { Tris } & \text { Tris(hydroxymethyl)-aminomethane } \\ \text { u } & \text { unit } \\ \text { Ubc } & \text { Ubiquitin C } \\ \text { UMC } & \text { University Medical Center } \\ \text { uNK } & \text { uterine natural killer cells } \\ \text { UV } & \text { ultraviolet } \\ \text { V } & \text { volt } \\ \text { v/v } & \text { volume/volume } \\ \text { VCAM-1 } & \text { vascular cell adhesion protein 1 (CD106) } \\ \text { vs. } & \text { versus } \\ \text { vw } & \text { ventricular weight } \\ \text { w } & \text { with } \\ \text { w/o } & \text { without } \\ \text { w/v } & \text { weight/volume } \\ \text { wt } & \text { wildtype } \\ \text { XX } & \text { female sex chromosomes } \\ \text { XY } & \text { male sex chromosomes } \\ \text { Zg16 } & \text { zymogen granule protein16 }\end{array}$


Introduction

\section{Introduction}

\subsection{Heart failure - a disease of considerable clinical and socioeconomic relevance}

Besides cancer, heart failure (HF) is one of the most important health problems worldwide with rising incidence in western societies due to aging of the population. The pandemic of HF reflects the achievement in preventing premature death caused by other diseases but affects 1-2 \% of the adult population in developed countries. In patients older than 70 years, HF is the major cause of disability and hospitalization with an increasing prevalence up to $10 \%$ in the population (Mosterd and Hoes, 2007). Current estimates assume that the number of deaths caused by HF will grow from todays 17.3 million to 23.6 million per year by 2030 (Mozaffarian et al., 2014). While cancer is considered as a gain of function disease, $\mathrm{HF}$ is a degenerative type of disease since cells become progressively dysfunctional and consequently tissue function is declined (Campisi, 2005). In general, HF is not a single diagnosis, but is rather a syndrome of multiple etiologies, which the American Heart Association defined as 'a complex clinical syndrome that results from any structural or functional impairment of ventricular filling or ejection of blood' (Yancy et al., 2013). Hereby, HF can be caused by a wide number of conditions, including ischemic heart disease (IHD, also coronary artery disease CAD; including e.g. myocardial infarction (MI)), arterial hypertension, valvular heart disease, primary cardiomyopathy, diabetes mellitus or infections and inflammation (myocarditis) (Korczyk et al., 2012). These processes lead to deregulation and impairment of interstitial and cellular elements in the heart including fibroblasts, extracellular matrix and myocytes, resulting in chronic, maladaptive ventricular remodeling. Here, the pathological remodeling includes an increased myocardial volume and mass due to ventricular dilation and a net loss of myocytes (Borghi et al., 2015). Initially, these changes are able to compensate for the damages of the myocardium but are later responsible for further progression into HF. A hallmark of $\mathrm{HF}$ is impaired contractility and one measure of this is a reduced ejection fraction (EF) (McMurray, 2010). 


\subsubsection{Therapy and molecular mechanisms of heart failure in humans}

Over the last decades, noninvasive and invasive techniques like echocardiography and cardiac catheterization were developed as most important diagnostic tools for patients with cardiovascular disease. However, despite large scientific efforts, the morbidity and mortality of HF remains high, since the molecular mechanisms leading to HF are still not clarified. Nevertheless, the identification of underlying targets is important for improving the efficacy of therapeutic strategies. The pathogenesis of chronic HF is often accompanied by inflammatory reactions. A cardiac stress response activates the innate immune system, which leads to expression of proinflammatory cytokines, including tumor necrosis factor alpha (TNF $\alpha$ ), Interleukin (IL)-1 and IL-6, as well as to the activation of Toll-like receptors (TLR), that detect pathogen-associated molecular patterns (PAMPs) and damage-associated molecular patterns (DAMPs). All features have been observed in patients with HF (Mann, 2003; Topkara et al., 2011). During the last years, studies of several laboratories demonstrated that the activation of the immune system plays an important role in the progression of cardiac remodeling in HF. It was observed that in wound-healing after cardiac injury such as $\mathrm{MI}$, the inflammatory response was crucial for the removal of the necrotic debris from the area of injury and helped to attract the cells involved in the formation of a scar (Blankesteijn and Altara, 2014). Besides this crucial contribution to initial tissue repair, a longstanding and strong inflammation can impair the heart function (Heymans et al., 2009). Hereby, inflammation has been described to be involved in the early development of cardiac hypertrophy as well as in later progression to HF (Ismahil et al., 2014; Koller et al., 2013; Purcell et al., 2001; Thaik et al., 1995; Yndestad et al., 2006).

\subsubsection{Evidence of autoimmunity}

The presence of autoimmune reactions accompanying the development to HF were monitored in several studies. In patients with myocarditis or dilated cardiomyopathy (DCM), several cardiac autoantibodies were identified, e.g. against $\alpha$ - and $\beta$-isoforms of cardiac myosin heavy chain (MyHC) or cardiac receptors such as $\beta 1$-adrenoreceptor (Magnusson et al., 1994). These anti-receptor antibodies were shown to either stimulate or block the receptor, thus affecting the cardiomyocyte contractility (Limas and Limas, 1991). Furthermore, it was shown that MI can lead to autoimmune reactions and the production of cardiac-specific antibodies of the immunoglobulin (Ig)G isotype (Bendjelid and Pugin, 2004). The presence of class-switched antibodies of the IgG isotype demonstrates an involvement of activated T helper cells with specificity for cardiac antigens. Several 
subgroups of Thelper cells were discussed to contribute to cardiac fibrosis due to cytokine secretion,

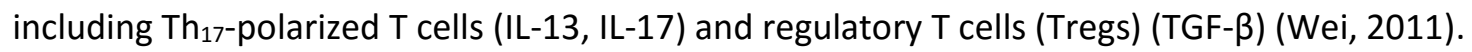

These observations highlight the importance of inflammation as a general molecular pathway of HF, offering potentially new options for therapy. Consequently, it is important to analyze the pathogenesis of the disease and to understand how inflammation or even autoimmunity could influence the success of new therapeutic options for HF, e.g. transplantations of stem cell-derived grafts into the heart.

The mechanisms underlying autoimmunity and specifically cardiac autoimmunity have not been fully clarified. Autoimmunity displays a failure of effective tolerance towards self-antigens by the adaptive immune system, leading to inflammation and degeneration of tissues or whole organs by an immunopathological process (Abou-Raya and Abou-Raya, 2006). Hereby, genetic as well as environmental factors contribute to the induction of the disease (von Herrath et al., 2003). As genetic factors, human leukocyte antigen (HLA) genotype, polymorphisms of cytokines and their receptors, or defective apoptosis genes can influence the responses to commonly encountered antigens (Actor, 2014). Additionally, variant antigen dose, infections, or exogenous influences causing tissue damage can lead to an altered immune response towards self-antigens (Ascherio and Munger, 2007; Fujinami, 2001; Libbey and Fujinami, 2010). Mechanisms for the loss of tolerance against self-antigens include failure of autoreactive $T$ cell deletion during development in the thymus, impairment of suppressor functions of Tregs, cross-reactivity between exogenous and self-antigens (molecular mimicry), increased B cell function based on polyclonal activation, e.g. by viral or bacterial components, enhanced or abnormal expression of MHC class II molecules, or release of self-antigens from so-called immunoprivileged sites. Consequently, various exogenous factors and/or genetic predispositions can lead to autoimmune reactions, which subsequently seem to play a role in secondary tissue damage, e.g. during HF (Figure 1). 


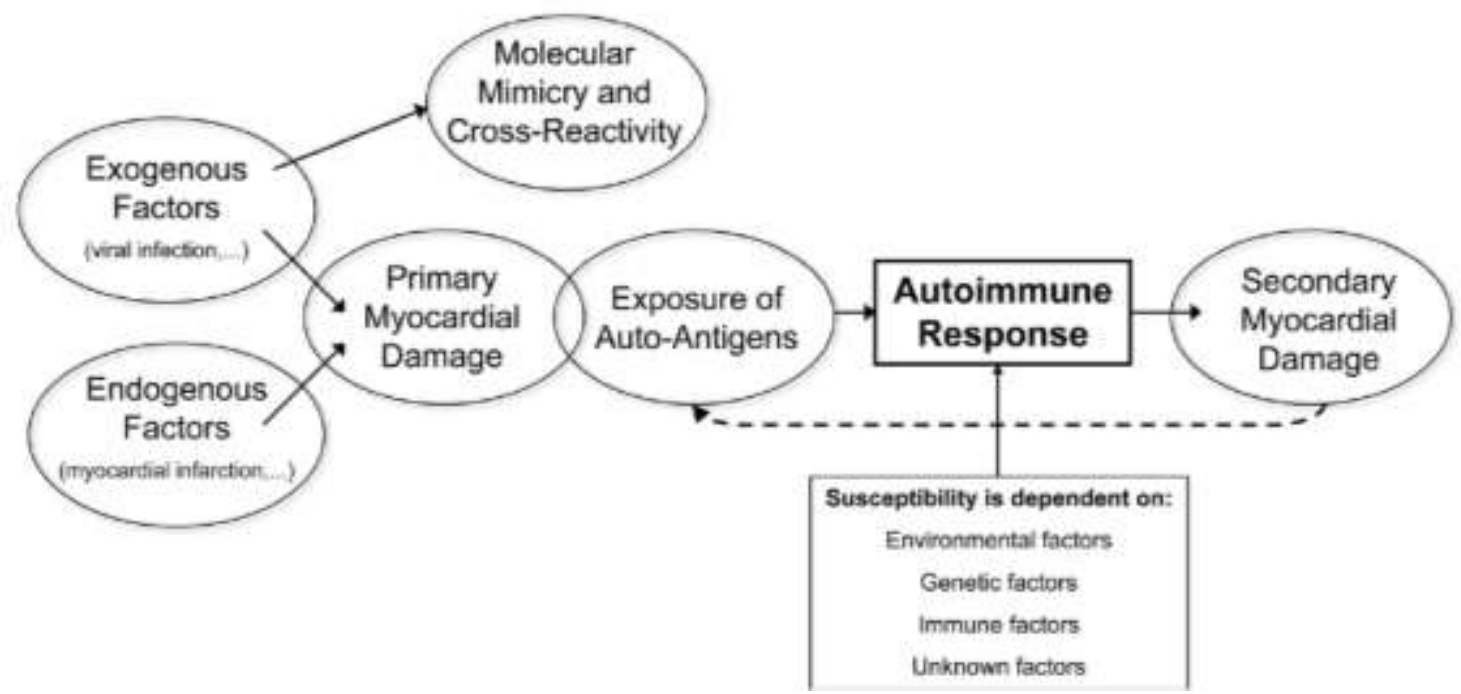

Figure 1 Potential mechanisms of autoimmunity induction after a primary myocardial damage, leading to the recognition of autoantigens by the immune system.

Subsequent autoimmune reactions could additionally harm the heart tissue and thus accelerating the progression to HF. At this, several factors or predispositions might play a role in the generation of autoimmunity in the heart. Picture by Kaya et al. (2012).

\subsubsection{Cardiac immunity}

\subsubsection{MHC and tolerance}

Besides various types of immune cells, which are responsible for the secretion of proinflammatory cytokines in autoimmunity, the primary cell types that are affected are B and T cells, recognizing selfantigens. Recognition of antigens by $\mathrm{T}$ cells is enabled by the MHC molecules. This extremely polymorphic gene complex encoding these molecules is the HLA in humans and H2 complex in mice. MHC molecules can be divided into two main types, MHC class I and MHC class II molecules, which differ in their function and expression pattern. All nucleated cells are able to express MHC class I molecules on their cell surface, allowing the identification of infected cells in the organism since, e.g. viral peptides are presented via the MHC class I molecules on infected cells to T cell receptors (TCR) on cytotoxic CD8 ${ }^{+} \mathrm{T}$ cells. If the T cell was activated before, this directly leads to killing of the target cells. In contrast, MHC class II molecules are solely expressed on professional antigen presenting cells (APCs) such as dendritic cells (DCs), macrophages, B cells and endothelial cells. The function of MHC class II molecules is to present peptides to the TCR of CD4 $4^{+}$helper T cells, which are central mediators of the adaptive immune response, triggering the secretion of cytokines and controlling the cellular 
and humoral defense. Hereby, the engagement of the TCR by a peptide-MHC complex is necessary for T cell activation to direct an effective adaptive immune response against invading pathogens. It is critical to ensure that the reactivity of the immune response to self-antigens is avoided. For this, central tolerance is induced already in immature lymphocytes during development of naïve T cells in the thymus where the rearrangement of $\alpha$ and $\beta$ TCR genes occurs and TCRs with random specificity are generated. T cells undergo two selection processes. In the first selection process, the positive selection, only T cells with a newly rearranged $\alpha \beta$ TCR that recognize MHC class I or class II molecules e.g. expressed on thymic epithelial cells, further mature into $\mathrm{CD}^{+}$and $\mathrm{CD} 4^{+}$single positive cells respectively. In the negative selection process, T cells with high affinity to self-peptides presented by MHC class I or class II molecules are eliminated by apoptosis. Those T lymphocytes that survive, complete their maturation process and are released as naïve $T$ cells into the circulation where further peripheral mechanisms of tolerance are able to suppress autoreactive T cells by induction of anergy (Figure 2). T cell reactivity generally requires two signals, an antigen-specific signal of the TCR recognizing peptide-loaded MHC molecules presented by APCs and a costimulatory signal from costimulatory molecules on the cell surface of professional APCs. CD28 is the best characterized costimulatory molecule on T cells, which interacts with CD80 and CD86 molecules on the cell surface of professional APCs. The absence of those costimulatory signals on the presenting cell leads to anergy of the T cell, meaning the T cell becomes functionally inactivated and is subsequently not capable of initiating proliferation or effector functions even if the antigen is encountered in the presence of full costimulation. Another mechanism of this peripheral tolerance induction can be caused by Tregs. Due to interactions including cytokine release, these cells can actively anergize lymphocytes in the periphery, which escaped the negative selection process in the thymus. Additionally, local secretion of immunosuppressive factors and presence of immunologically privileged sites (e.g. brain and eyes) can be seen as tolerance mechanisms due to the exclusion of antigen excess by a physical barrier. However disruption of the latter due to tissue damage can lead to release of hidden (cryptic) antigens, potentially leading to lymphocyte activation. 


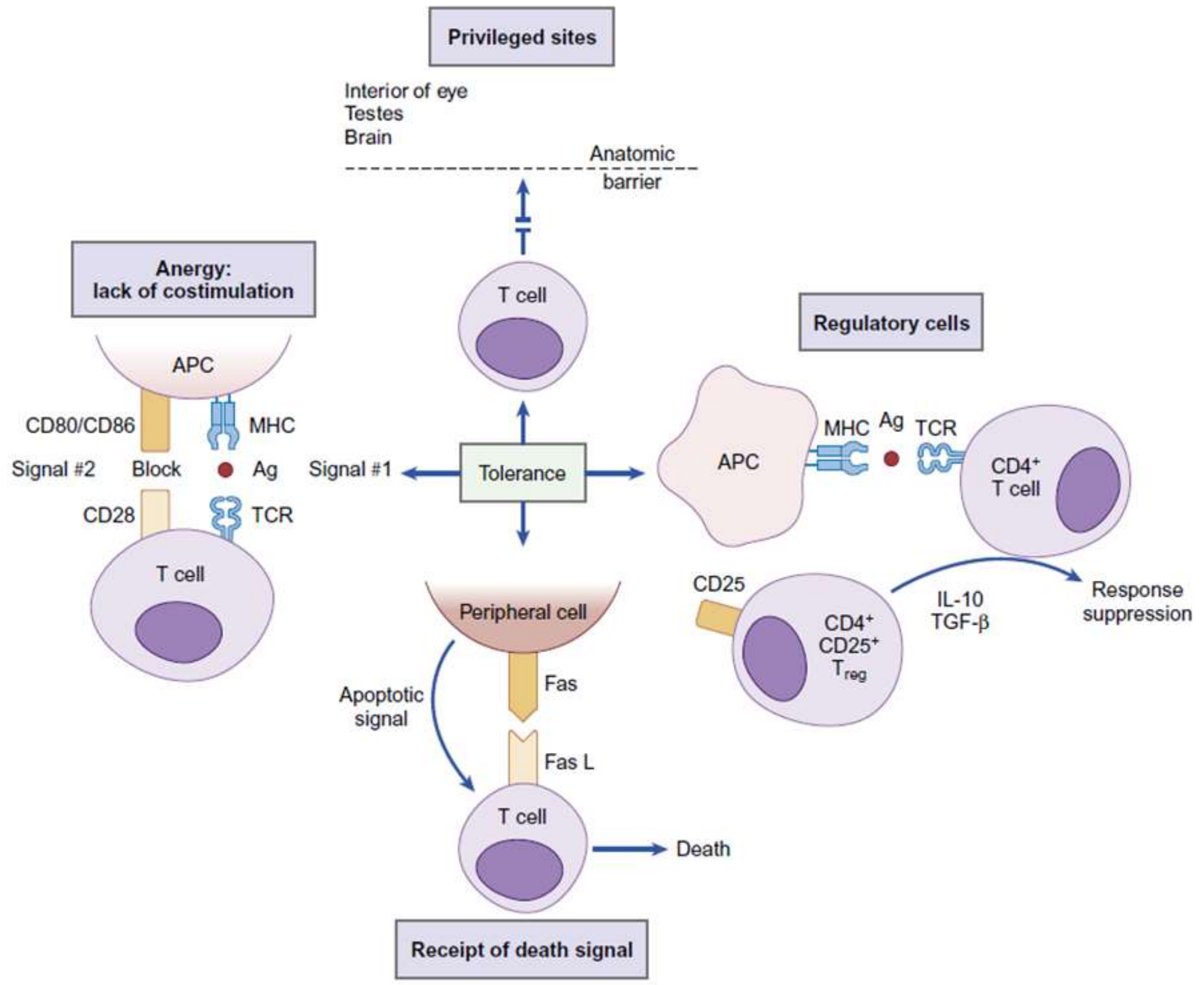

Figure 2 Tolerance induction of $\mathrm{T}$ lymphocytes in the thymus and periphery.

Central tolerance comprises negative selection in the thymus, leading to apoptosis of autoreactive $T$ cells. Peripheral tolerance includes induction of anergy due to absence of costimulatory signals, active suppression by Tregs via cytokine secretion or the interception of antigens in immunoprivileged sites. Antigen (Ag), L (ligand). Picture by Actor, 2014).

B lymphocytes undergo central tolerance during their development in the bone marrow where the immature $B$ cells undergo apoptosis upon interaction of IgM with multivalent self-molecules like MHC molecules expressed on stromal cells (clonal deletion). However, alternatively B cell can be rescued by receptor editing through further $B$ cell receptor $(B C R)$ gene rearrangements, replacing self-reactive B cell receptors. B cell anergy is induced by exposure to soluble circulating antigens, which lead to a downregulation of surface IgM expression and partial blockade of the intracellular signaling cascade. Generally, only B lymphocytes without self-reactivity migrate to the periphery albeit leakage of weakly self-reactive (sleeper) B cells can occur. These cells normally do not cause problems because they require help by antigen-specific T cells to become activated. However, they might become activated 
under certain conditions such as inflammation. In the periphery, response of B cells to antigens depends on its isotype type and signaling of coreceptors such as CD19 and CD21. T cell-dependent activation of B cells requires a TCR-MHC-II peptide binding complex and interaction of costimulatory signals like CD40L on T cells and the B cell surface receptor CD40 leading to B cell proliferation, immunoglobulin class switching and somatic hypermutation. Additional cytokines released by $\mathrm{T}$ cells bind to cytokine receptors of B lymphocytes and promote these processes.

\subsubsection{HF in animal models}

\subsection{Animal models}

Animal models remain an invaluable implement to study cardiovascular diseases. Besides the advantage of reasonable similarities in biochemical pathways, pharmacological targets and organ function, mice are easy to keep, breed and relatively cheap in maintenance. The broad range of available invasive and non-invasive techniques including magnetic resonance imaging (MRI), hemodynamic measurements, electro- and echocardiography allow the evaluation of heart function, infarct size and specification of cardiac remodeling. Additionally, genetic engineering enables further examination of various cardiovascular diseases by mimicking its pathogenesis or addressing fundamental mechanism in transgenic or knockout strains. Consequently, mouse models could reveal future therapeutic strategies or molecular targets to treat HF.

\subsection{Murine overload models to induce HF}

Conventional methods to induce HF in mice include surgery to increase the load of the murine heart. These can be divided in volume or pressure overload models of HF that increase either the preload or the afterload, respectively. In contrast to an increased blood volume (preload), that can be caused by renal failure or valve insufficiency, an increased afterload displays a higher vascular resistance that can be obtained by hypertonus, vascular or valvular stenosis. The most common model of pressure overload is the transverse aortic constriction (TAC), which was first described by Rockman et al. (1991). Hereby, HF is induced by a partly constriction of the aorta, leading to an increased pressure gradient in the heart that can be quantified across the stricture by echocardiography. Subsequently, the heart becomes hypertrophic within 2 weeks, showing an increase of left ventricular (LV) mass of about $50 \%$ (Figure 3). 
A.

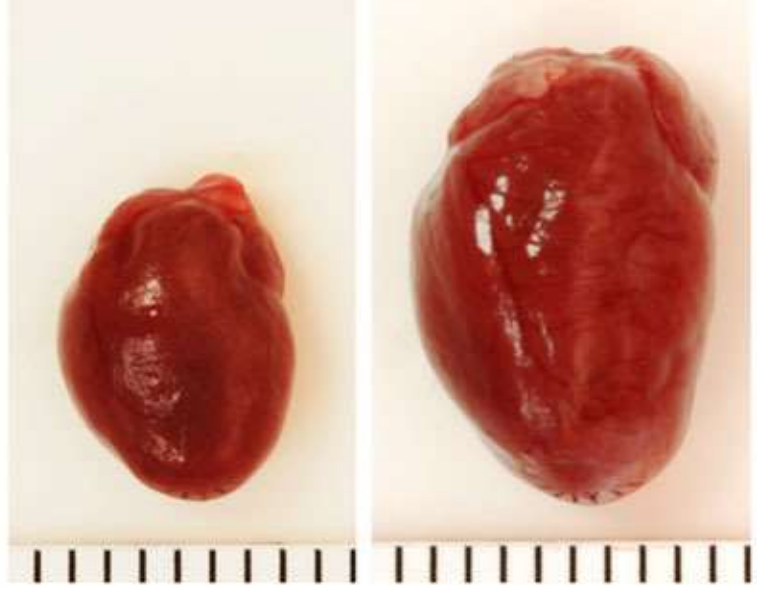

Figure 3 Exemplary pictures of (A) sham- and (B) TAC-operated hearts of C57BL/6 mice 16 weeks after the operation.

TAC operation led to cardiac remodeling and hypertrophy of the heart. Scale bars indicate 1 $\mathrm{mm}$ each line. Picture by deAlmeida et al. (2010).

In accordance with findings in human pathology, several animals studies in rats and mice revealed an influence of inflammation on the development of cardiac hypertrophy (Ismahil et al., 2014; Purcell et al., 2001; Thaik et al., 1995). The investigation of murine cardiac remodeling in a preload (aortocaval shunt) and afterload (TAC) model in C57BL/6 mice revealed an increase of leukocyte infiltration, fibrosis, and apoptosis in response to TAC (Toischer et al., 2010). Additionally, expression profiling data suggested an increased activity of T cells, B cells and natural killer (NK) cells in the afterload model in contrast to the preload model (aorto-caval shunt). Consequently, our research group focused on the examination of immune reactions after TAC operations in C57BL/6 mice. It was monitored that the proportion of myeloid cells and lymphocytes in the myocardium was increased after TAC as determined by immunohistochemistry (IHC) and flow cytometry (Sasse et al., unpublished data) Furthermore, it was shown that NK cells, T helper and cytotoxic T cells were systemically activated, leading to a severe inflammation in single animals (Sasse et al., unpublished data). By others, it was shown that humoral autoimmune reactions occurred in HF models, e.g. antibodies against troponin I, a cardiac-specific antigen, were found in mice (Kaya et al., 2008). Additionally, cardiomyocyte-specific autoantibodies were identified in a rat model of aortic constriction (Liu et al., 2002) and in mouse and rabbit models (Kaya et al., 2012). These findings indicate a link of autoantibodies to HF and suggest that also activated $\mathrm{CD} 4^{+} \mathrm{T}$ cells with specificity for cardiac antigens might play a role in ventricular remodeling and the progression from cardiac hypertrophy to HF. Nevertheless, these autoreactive T cells had not been directly identified in these animal models, yet. Analysis of immunodeficient mice showed that these are not protected from the progression to HF after TAC surgery (Sasse et al., unpublished data). It was recently shown that OT-II mice, which only bear TCR-transgenic T cells 
specific for ovalbumin (OVA), which is not present in the murine organism under normal conditions, failed to develop HF and adverse remodeling after TAC (Laroumanie et al., 2014). This is a further hint that antigen-specific immune responses in the heart could be a critical for the progression to HF. We further analyzed this process in our research group. CMy-mOVA mice that express OVA selectively in cardiomyocytes are a suitable model to study the influence of antigen-specific immune reactions and autoimmunity on the outcome of HF. These mice express OVA under the control of the mouse cardiac alpha myosin heavy chain (MyHC) promoter on the cell surface of cardiomyocytes (Figure 4). They were first described by Grabie et al. (2003a), who showed that the endogenous adaptive immune system of cMy-mOVA mice displays tolerance to OVA.

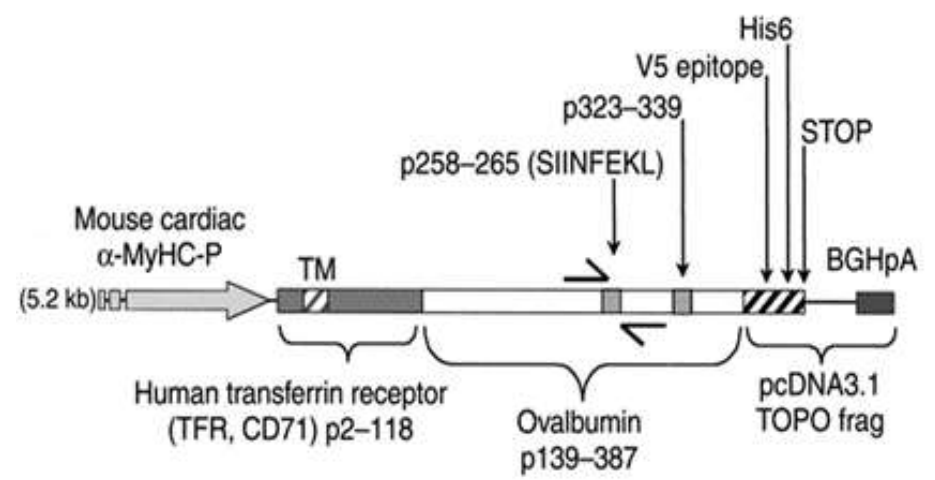

Figure 4 CMy-mOVA transgene construct. OVA 258-265 and OVA 323-339 epitopes are recognized by OT-I and OT-II TCRtransgenic $\mathrm{T}$ cells, respectively. BGHpA, bovine growth hormone, polyadenylation signal; TM, transmembrane domain; frag, DNA fragment. Picture by Grabie et al. (2003).

To test if these mice develop autoimmunity against OVA in HF, they underwent TAC surgery. After this, neither OVA-specific IgG autoantibodies arose nor was the proliferation or cytokine expression pattern of $\mathrm{CD}^{+} \mathrm{T}$ cells altered compared to sham-operated mice (Röhrborn et al., unpublished data). In contrast, a low but significant activation of OVA-specific $\mathrm{CD}^{+} \mathrm{T}$ cells was found 10 weeks after TAC, but this did not significantly impair heart function. However, single animals displayed a higher activity of OVA-specific CTLs. These findings suggested that clinically relevant autoimmunity against OVA as cardiac antigen is not frequent after TAC in young and otherwise healthy mice. However, cytotoxic T cells were activated at low level and consequently, in this thesis, the role of T cells in animals, which are at higher risk to develop autoimmunity was further investigated. Therefore, we challenged the cMy-mOVA mice with adoptive transfer of OVA-specific $\mathrm{CD}^{+}$and $\mathrm{CD} 8^{+} \mathrm{T}$ cells and aimed to generate double-transgenic cMy-mOVA/OT-I and cMy-mOVA/OT-II mice, which were expected to have predominantly OVA-specific $\mathrm{CD}^{+}$or $\mathrm{CD}^{+} \mathrm{T}$ cells. The influence of these OVA-specific $\mathrm{T}$ cells on the progression to $\mathrm{HF}$ and on the immunological milieu in the myocardium of cMy-mOVA mice was 
examined. The heart function was assessed by echocardiography and immunological test were performed to determine immune responses against OVA and the infiltration of immune cells into the myocardium and pathologic features like fibrosis were evaluated.

\subsubsection{Importance for stem cell therapy}

The immunological characterization of the failing myocard is highly important for the development of new therapeutic strategies such as the transplantation of cardiomyocytes derived from pluripotent stem cells (PSCs). These transplantations are an upcoming therapeutic concept for HF. Although being a promising therapeutic approach, its success in pre-clinical models is currently limited due to loss of implanted cells and poor integration (Laflamme and Murry, 2011). Most of the integrated cells either spontaneously die or are eliminated by the immune system of the recipient. These problems are presumably aggravated by a proinflammatory milieu in the target tissue. Thus, the immunological situation in the target tissue of a stem cell-derived transplant has to be considered in the development of new transplantation therapies. Moreover, although induced pluripotent stem cell (iPSC) technology principally could allow the generation of autologous grafts, most researchers and physicians in the field of regenerative medicine currently assume that only allogeneic transplantations will be feasible for indications such as HF due to economic and time restrictions. 


\subsection{Stem cell therapy}

The possibility of using PSCs to replace damaged cells or tissue is very appealing and has been suggested for various diseases including chronic HF. The replacement of diseased organs by donor organs requires lifelong immunosuppressive treatment. Moreover, more organs are required than are available. Nonetheless, the frequency of age-related diseases is rising due to the increasing age of populations in western countries. Thus, the development of alternative treatments including transplantation of stem cells or stem cell-derived grafts is required. However, these regenerative therapies will also likely encounter immunological hurdles. The use of allogeneic cells could lead to rapid immune rejection if the $\mathrm{MHC}$ molecules of donor and recipient are mismatched. If $\mathrm{MHC}$ molecules are matched, minor histocompatibility $(\mathrm{miHC})$ antigens could still lead to graft rejection by the immune system of the recipient. Another hurdle is the ability of PSCs to indefinitely proliferate and to give rise to teratomas if residuals of pluripotent cells remain in a graft after in vitro differentiation. Here, the immune system could reduce tumor risk, if specifically pluripotent cells could be targeted.

\subsubsection{Stem cell types and their role for transplantation therapy}

Embryonic stem cells (ESCs) were the first isolated and cultured PSCs, obtained from mouse embryos in 1981 by Evans and Kaufman, and Martin, and later from human embryos by Thomson et al. (1998). ESCs are cells derived from the inner cell mass (ICM) of the early blastocyst. They have the capacity of self-renewal and to differentiate into any cell type of the three germ layers (Bradley et al., 1984). However, because of ethical concerns about their generation and the limitation to be used for the generation of allogenic grafts only, efforts to generate pluripotent cells that overcome these limitations were pushed. The strategies for the development of autologous pluripotent stem cells included somatic cell nuclear transfer (SCNT) and the generation of iPSCs. However, the generations of PSCs by SCNT, in which a patient-specific nucleus is transferred into an enucleated oocyte, involves again usage of ethical debated biological materials (Rao and Condic, 2008). Therefore, the method of reprogramming patient-specific somatic cells into PSCs by transduction of specific transcription factors was rapidly further investigated and developed since their first description by Takahashi and Yamanaka (2006). Subsequently, iPSCs have been generated from rat (Buehr et al., 2008), rabbit (Honda et al., 2010), pig (Esteban et al., 2009), dog (Koh et al., 2010), monkey (Liu et al., 2008) and human (Takahashi et al., 2007; Yu et al., 2007). Initially, murine fibroblast were reprogrammed by the 
transcription factors Oct4, KIf4, Sox2 and c-Myc. Human iPSCs were generated by introducing these factors (Takahashi et al., 2007) or OCT4, SOX2, NANOG, and LIN28 (Yu et al., 2007) into somatic cells. IPSCs were generated from several cell types including keratinocytes, hepatocytes, hematopoietic cells, pancreatic cells and also from patients, including those with Parkinson's disease (PD), down syndrome (DS) and Huntington disease (HD) (Park et al., 2008; Takahashi and Yamanaka, 2006). The therapeutic potential of iPSCs was shown in several animals studies, in which murine iPSCs were successful differentiated into neural, cardiovascular, hematopoietic or hepatic progenitor cells (Cantz et al., 2008; Kuzmenkin et al., 2009; Narazaki et al., 2008; Schenke-Layland et al., 2008; Wernig et al., 2008). Especially the differentiation of PSCs into functional cardiomyocytes promoted therapeutic studies treating the diseased myocardium (Caspi et al., 2007; Laflamme et al., 2007; Qian et al., 2012; Shiba et al., 2012; Singla et al., 2006; Song et al., 2012; Xue et al., 2005). Due to these innovations, stem cells are seen not only as regenerative source for patient-specific cell therapies but could also replace animal experiments in toxicity tests and drug development. Nonetheless, despite the promising results, PSC technology has to overcome various obstacles.

\subsubsection{Limitations}

The usage of viral vectors such as lentivirus and retrovirus was reported to cause tumors and genomic instabilities due to the integration of the transcription factors into the genome (Takahashi and Yamanaka, 2006). Therefore, various non-integration techniques including the use of plasmids, small molecules, mRNA, miRNA, recombinant proteins or adenovirus vectors were examined to improve the reprogramming method (Anokye-Danso et al., 2011; Fusaki et al., 2009; Okita et al., 2008; Rohani et al., 2016; Soldner et al., 2009; Yakubov et al., 2010; Yusa et al., 2009). Additionally, analysis of differentiation, gene expression and epigenetic modulation revealed an frequently insufficient reprogramming of iPSCs (Ghosh et al., 2010; Hu et al., 2010; Marchetto et al., 2009; Pick et al., 2009), which has to be considered for future therapeutic applications. These differences in the PSC profile could sustain after in vitro differentiation and might lead to unexpected immune responses in the recipient, eventually destroying the graft and harming the recipient. Moreover, the general immunogenicity of PSCs has to be analyzed before they can be used for the clinic. 


\subsubsection{Stem cell immunogenicity}

\subsubsection{Expression of histocompatibility antigens}

Histocompatibility antigens can be divided into $\mathrm{MHC}$ molecules, miHC antigens and $\mathrm{ABO}$ blood group antigens (Roopenian et al., 2002, Watkins, 2001). Among these variable antigens, the highly polymorphic MHC molecules are the strongest indicator for inducing allograft rejections after transplantation, if mismatched between donor and recipient (Lechler et al., 2005). MHC molecules present processed antigens to circulating $T$ cells which normally respond only to foreign antigens, due to tolerance induction in the thymus or periphery. MHC class I molecules, expressed on nearly all nucleated cells, present primarily cytosolic proteins to $\mathrm{CD} 8^{+} \mathrm{T}$ cells, whereas $\mathrm{MHC}$ class II molecules, located on the surface of professional APCs, show exogenous antigens to $\mathrm{CD}^{+} \mathrm{T}$ cells. The allorecognition pathways can be divided into the direct and indirect pathway, which are mainly mediated by professional APCs and T cells (Figure 5). During direct allorecognition, T cells recognize intact allogeneic MHC molecules on donor APCs such as DCs that migrated from the graft to lymph nodes of the recipient. T cell activation results in acute cytotoxic responses leading to graft rejection (Morelli and Thomson, 2003). In contrast, the indirect allorecognition pathway comprises the recognition of processed alloantigens by T cells in the context of recipient MHC class II molecules, causing chronic rejection by promoting the production of alloantibodies and allospecific $\mathrm{T}$ cells (Briscoe and Sayegh, 2002; Morelli and Thomson, 2003).

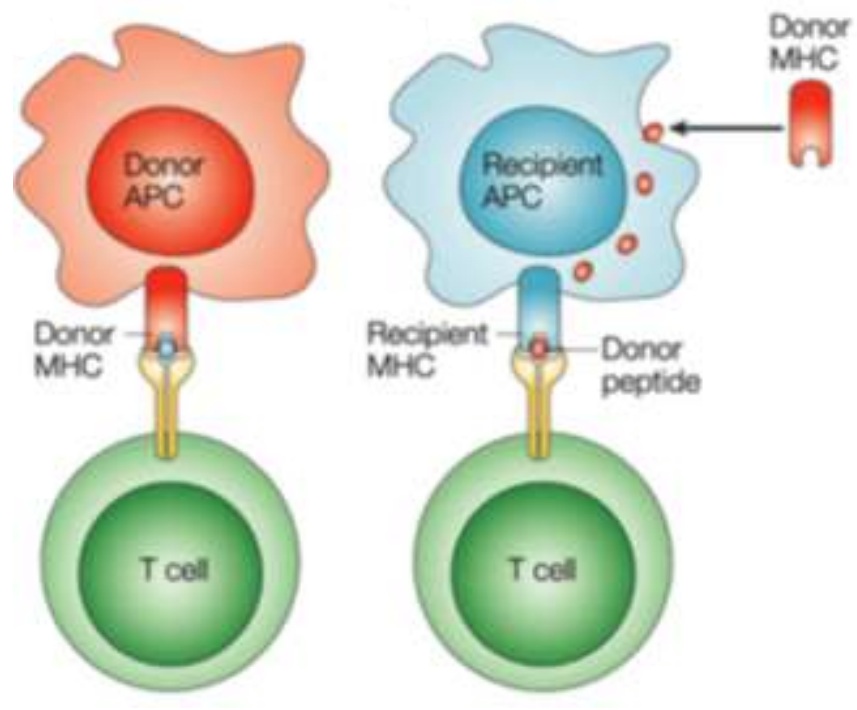

Figure 5 Mechanism of allorecognition. During direct allorecognition (left), T cells recognize foreign $\mathrm{MHC}$ molecules on donor APCs, whereas the indirect allorecognition (right) involves the degradation and processing of donor $\mathrm{MHC}$ molecules or other allogenes to peptides which are taken up by recipient APCs and presented to T cells. Picture by Bradley et al. (2002). 
Consequently, it is preferred to match MHC alleles prior to organ transplantation to reduce the immune response against allografts. However, these processes cannot only be elicited by foreign MHC molecules, but by any other polymorphic antigens, collectively known as miHC antigens. Generally, one third of newly synthesized proteins is immediately degraded by the proteasome, producing peptides that can potentially elicit $\mathrm{CD}^{+}$and $\mathrm{CD}^{+} \mathrm{T}$ cell alloresponses, if the proteins display polymorphism between individuals in a transplantation setting, even if $\mathrm{MHC}$ loci were matched between donor and recipient (Roopenian et al., 2002; Wallny and Rammensee, 1990). Those reactions were shown after transplantations between HLA-identical siblings in which the recipients developed graft versus host disease (GVHD), graft versus leukemia (GVL) or T cell-mediated graft failure (Bleakley and Riddell, 2004; Ferrara et al., 2009; Goulmy et al., 1976; Opelz and Collaborative Transplant Study, 2005). Also, if patient-specific iPSC would be used for transplantation, it is still possible that miHC antigens are ectopically expressed due reprogramming or in vitro culture (Dhodapkar et al., 2010; Zhao et al., 2011). Nevertheless, to which extent these miHC antigens would affect immune responses after transplantations of stem cell derived grafts, has to be clarified.

To evaluate the antigenic characteristics of PSCs, expression of MHC molecules on human and murine PSCs were examined during the last years. Generally, it was shown that human PSCs express only low levels of MHC class I molecules but no MHC class II molecules (Draper et al., 2002; Drukker et al., 2002; Jurisicova et al., 1996). On murine PSCs, no MHC class I or class II molecules were detected via flow cytometry (Dressel et al., 2008, 2009; Magliocca et al., 2006; Tian et al., 1997). Nevertheless, minor amounts of MHC class I molecules must to be expressed since murine ESCs were shown to become targets of CTLs after pulsing with appropriate peptides (Dressel et al., 2009). Some PSCs were reported to upregulate MHC class I molecules after the treatment with the proinflammatory cytokine interferon $\gamma$ (IFNY) but MHC class II molecules were not detected (Bonde and Zavazava, 2006; Drukker et al., 2002). However, other murine studies showed that MHC class I molecules on PSCs were not affected by INFy treatment (Abdullah et al., 2007; Monecke, 2013; Nussbaum et al., 2007; Tian et al., 1997). With differentiation, levels of MHC class I molecules increased (Drukker et al., 2002; Nussbaum et al., 2007) and MHC class II expression was ascertained after DC and hematopoietic differentiation (Senju et al., 2007; Slukvin et al., 2006; Zhan et al., 2004).

Concerning the $A B O$ antigen expression, it was demonstrated that human ESCs and differentiated cardiomyocyte-like cells express these antigens, what has to be considered prior to clinical translation (Mölne et al., 2008). 
Expression of miHC antigens on PSCs was not directly investigated to date, albeit OCT4-specific T cells were found in humans, which is a key factor for pluripotency in stem cells (Dhodapkar et al., 2010). Nonetheless, whether the expression of miHC antigens on PSCs alter their immunogenicity has to be analyzed, which will be done in this thesis by using OVA as model antigen expressed in PSCs.

\subsubsection{Susceptibility to $\mathrm{T}$ and NK cell killing}

Besides MHC-TCR interaction, costimulatory signals are important for T cell-mediated immune responses. CD80 and CD86, ligands for the activation receptor CD28 on T cells were not expressed on human ESCs (Drukker et al., 2002; Lechler et al., 2005) but were shown after differentiation into hematopoietic cells or DCs (Senju et al., 2007; Slukvin et al., 2006; Zhan et al., 2004). Adhesion molecules such as the intercellular adhesion molecule 1 (ICAM-1) and ICAM-3, or B7 molecules (Suh et al., 2003) might have additional important functions for PSC immunogenicity. ICAM-1 was reported to be important for the killing of murine ESCs (Frenzel et al., 2009).

However, ligands negatively regulating T cells such as Fas ligand (FasL), which recognizes the death receptor Fas on lymphocytes and leads to apoptosis, was shown only on rat ES-like cells but not on human PSCs (Drukker, 2006; Fändrich et al., 2002). Additionally, expression of Serpin-6, an inhibitor of cytotoxic T cells was found on murine ESCs (Abdullah et al., 2007). However, our group could not confirm these results due to missing surface expression of Serpin- 6 on several murine PSC lines and small amount of detected mRNA (Dressel et al., 2010). The protective protein Cathepsin B, which inactivates granzyme B (GrB), was detected on various murine PSCs, but showed no functional correlation with resistance to CTLs (Dressel et al., 2010). Additionally, Arginase 1 (Arg1) and Indoleamine 2, 3-dioxygenase (IDO), enzymes that degrade necessary amino acids from the microenvironment, thereby inhibiting $T$ cells activation and proliferation were reported to be expressed on PSCs in few studies (Plumas et al., 2005; Su et al., 2014; Yachimovich-Cohen et al., 2010). However, they could not be confirmed to be expressed on murine PSCs by prior studies in our group (Monecke, 2013). Furthermore, it was reported that murine ESCs evade an immune response by secretion of transforming growth factor beta (TGF $\beta$ ), which generally inhibits the activation of naïve T cells (Koch et al., 2008). 
Our group showed that PSCs were able to actively suppress T cell activation in vitro, mediated by cellcell contact of PSCs and T cells, but the cellular mechanism could not be clarified (Monecke, 2013). However, it was shown that the rejection of murine ESCs is dependent on the immune competence of the recipient and the immunogenetic setting of the transplantation and includes several immune effector mechanisms such as T cells and the complement system (Dressel et al., 2008; Koch et al., 2008; Pearl et al., 2011). Moreover, T cell-mediated responses against murine ESCs were demonstrated in several in vivo studies (Boyd and Wood, 2009; Robertson et al., 2007; Wu et al., 2008) and despite the low expression of MHC class I molecules, it was shown that CTLs can kill PSCs in a peptide-dependent manner (Dressel et al., 2009). However, murine PSCs fail to process endogenous antigens and require pulsing with exogenous peptides to be recognized by CTLs (Monecke, 2013).

NK cell activation against target cells is regulated by signaling of inhibitory and activating receptors on the surface of the cells (Figure 6) (Koch et al., 2013; Raulet, 2006). MHC class I molecules serve as ligands for Ly49 receptors on murine and for killer immunoglobulin-like receptors (KIRs) on human NK cells (Pegram et al., 2011). The majority of these receptors are inhibitory and signal through immunoreceptor tyrosine-based inhibitory motif (ITIM), however also activating KIRs and Ly49 receptors are known. Further NK cell receptors include the activating receptor NKG2D, which interacts with MICA, MICB and ULBPs (1-6) ligands on human cells, and with RAE1, MULT1 and H60 ligands on murine cells. Other activating receptors are DNAM-1 to which CD122 and CD155 ligands can bind and the natural cytotoxicity receptors (NCRs), including NKp30, NKp44 and NKp46, for which a number of various ligands were described (Koch et al., 2013). Generally, NK cell receptors like MICA and MICB are not expressed by normal cells but become upregulated due to cellular or genotoxic stress in diseased or infected cells, leading to NK cell activation by the 'stress-induced self' concept (Vivier et al., 2012). 


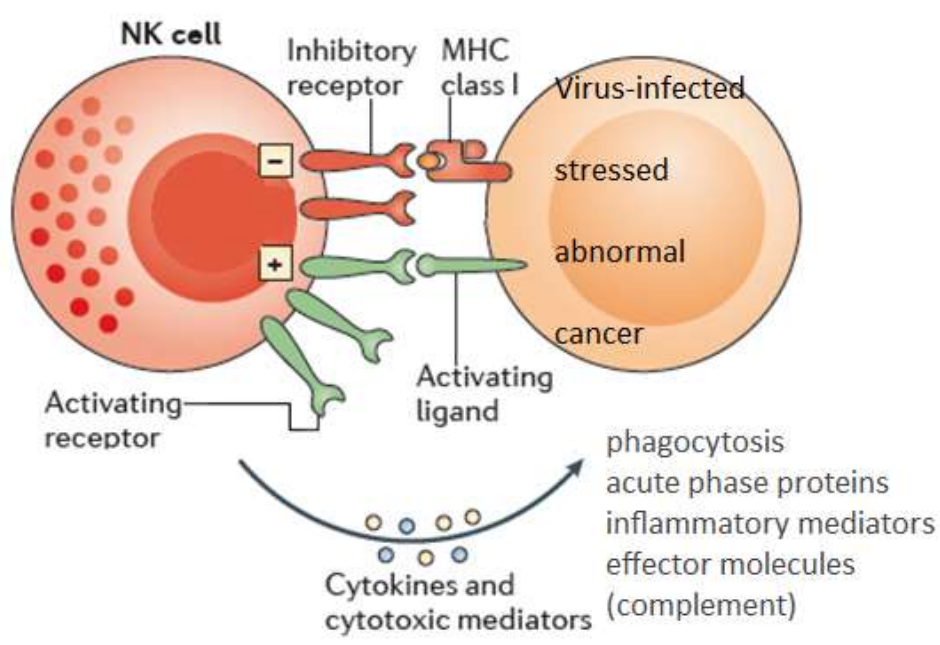

Figure 6 The activation of NK cells depends on a balance between inhibitory and activating receptor signaling.

The lack of MHC class I molecules can trigger the cytotoxic activity of NK cells, which is described as missingself concept (Kärre, 2008). Additionally, upregulation of activating receptors on diseased or stress cells can shift the balance toward NK cell activation. Picture modified from Vivier et al. (2012).

Despite several studies in which it was claimed that ESCs are resistant to NK cells (Drukker, 2006; Nussbaum et al., 2007; Swijnenburg et al., 2005), it was shown that various PSC lines, including murine ESCs, iPSCs and maGSCs can become targets of NK cells due to high expression of ligands for activating NK cell receptors, such as NKG2D and DNAM-1, and low expression of ligands for inhibitory NK receptors, such as MHC class I molecules (Dressel et al., 2008, 2010; Frenzel et al., 2009; SuárezAlvarez et al., 2010). This includes expression of low amounts of ligands of the activating NK receptor NKp46 and the NKG2D ligands MICA and MICB detected on human ESCs and iPSCs (Chen et al., 2015; Suárez-Alvarez et al., 2010). Murine and human PSCs were shown to be highly susceptible to killing by IL-2-activated allogeneic and autologous NK cells (Dressel et al., 2008, 2010; Elsner et al., 2010; Kruse, Hamann et al., 2015). At this, killing of human PSCs was partly dependent on the activating NK receptor DNAM-1, whereas in the murine situation NKG2D seemed to play a major role.

In summary, the recent results showed that PSCs are more immunogenic than initially proposed (Koch et al., 2008; Li et al., 2004b; Magliocca et al., 2006). Nevertheless, extensive investigations about the impact of miHC antigen expression on the stem cell immunogenicity are still lacking. Therefore, in vivo analysis of the previously in vitro examined OVA-expressing stem cells (Monecke, 2013) were performed to determine the immunogenic potential of those miHC antigen expressing stem cells compared to wildtype stem cells. 


\section{Aims of this thesis}

The major objectives of this thesis were to examine (1) T cell immune reactions against a cardiomyocyte-specific antigen during the progression to heart failure (HF) and (2) T cell immune reactions against an antigen of stem cells or stem cell-derived grafts that might affect transplantation of those grafts. Knowledge of the immunological milieu in the myocardium is important for future medical therapies including tissue transplantation or control of autoimmunity to counteract tissue or graft damage by suitable treatments.

\section{Occurrence of autoimmunity during heart failure}

In the first part of the thesis, the development of autoimmunity in a model of increased afterload leading to HF in mice at risk for autoimmune responses was examined. Important questions to answer were whether autoimmunity can be triggered by an increased afterload of the heart and additional challenge by autoreactive T cells. Differences in the progression to HF or in the immune response after transfer of autoreactive T cells or endogenous autoreactive T cells should be clarified.

\section{Influence of minor histocompatibility antigen expression on stem cell immunity}

Due to the expression of OVA as endogenously expressed antigen in stem cells, the effect of a single miHC antigen on engraftment and stem cell immunogenicity was analyzed. For this, teratoma formation in immunodeficient and immunocompetent syngeneic recipients was accessed, $T$ and $B$ cell activation against OVA monitored, and their infiltration into stem cell-derived teratomas determined.

\section{Clarification of importance of NKG2D for NK cell killing of pluripotent stem cells}

In addition to T cells also NK cells contribute to the rejection of PSCs. Inhibition experiments in our research group had identified NKG2D as the most relevant NK cell receptor for recognition of murine PSCs, but DNAM-1 as most important for killing of human iPSCs. Therefore, we wanted to examine the relevance of NKG2D for killing of murine PSCs by NK cells by comparing NKG2D-deficient and wildtype NK cells. 


\section{Materials and Methods}

\subsection{Materials}

\subsubsection{Biological material}

\subsubsection{Cell lines}

Table 1 Cell lines.

\begin{tabular}{|c|c|c|c|c|}
\hline Type & Label & Origin & Description & Reference \\
\hline \multirow{8}{*}{ 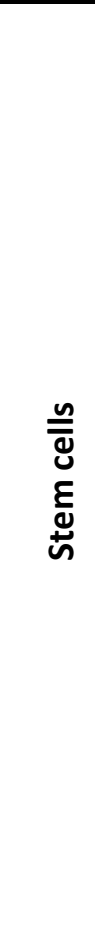 } & ESC BTL1 & $\begin{array}{l}129 / \mathrm{Sv} \\
\text { mouse }\end{array}$ & $\begin{array}{l}\text { blastocyst-derived } \\
\text { embryonic stem cell }\end{array}$ & $\begin{array}{l}\text { W. Engel; Human } \\
\text { Genetics, UMC } \\
\text { Göttingen. }\end{array}$ \\
\hline & ESC MPI-II & $\begin{array}{l}129 / \mathrm{Sv} \\
\text { mouse }\end{array}$ & $\begin{array}{l}\text { blastocyst-derived } \\
\text { embryonic stem cell }\end{array}$ & $\begin{array}{l}\text { A. Mansouri; MPI for } \\
\text { Biophysical Chemistry, } \\
\text { Göttingen }\end{array}$ \\
\hline & $\begin{array}{l}\text { ESC BTL1 OVA } \\
\text { (clones } \# 1, \# 4, \\
\# 9, \# 14, \# 20 \text { ) }\end{array}$ & $\begin{array}{l}129 / \mathrm{Sv} \\
\text { mouse }\end{array}$ & $\begin{array}{l}\text { hEF1 } \alpha / C A G-P-O V A-e G F P- \\
\text { Neo }^{R}\end{array}$ & (Monecke, 2013) \\
\hline & $\begin{array}{l}\text { ESC MPI-II OVA } \\
\text { (clones \#1, \#4) }\end{array}$ & $\begin{array}{l}129 / \mathrm{Sv} \\
\text { mouse }\end{array}$ & $\begin{array}{l}\text { hEF1 } \alpha / C A G-P-O V A-e G F P- \\
\text { Neo }^{R}\end{array}$ & (Monecke, 2013) \\
\hline & iPSC (129/Sv) & $\begin{array}{l}129 / \mathrm{Sv} \\
\text { mouse }\end{array}$ & $\begin{array}{l}\text { iPSCs; clone 11.1; derived } \\
\text { from MEFs }\end{array}$ & (Guan et al., 2006) \\
\hline & $\begin{array}{l}\text { iPSC } 129 / \text { Sv OVA } \\
\text { (clones \#6, \#13, } \\
\# 18, \# 24 \text { ) }\end{array}$ & $\begin{array}{l}129 / \mathrm{Sv} \\
\text { mouse }\end{array}$ & $\begin{array}{l}\text { hEF1 } \alpha / C A G-P-O V A-e G F P- \\
\text { Neo }^{R}\end{array}$ & (Monecke, 2013) \\
\hline & $\operatorname{maGSC}(129 / \mathrm{Sv})$ & $\begin{array}{l}129 / \mathrm{Sv} \\
\text { mouse }\end{array}$ & $\begin{array}{l}\text { multipotent adult } \\
\text { germline stem cell }\end{array}$ & (Guan et al., 2006) \\
\hline & maGSC (C57BL/6) & $\begin{array}{l}\mathrm{C} 57 \mathrm{BL} / 6 \\
\text { mouse }\end{array}$ & $\begin{array}{l}\text { multipotent adult } \\
\text { germline stem cell }\end{array}$ & (Guan et al., 2006) \\
\hline \multirow{3}{*}{ 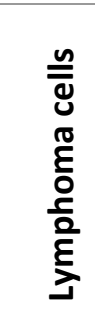 } & RMA eGFP & $\begin{array}{l}\mathrm{C} 57 \mathrm{BL} / 6 \\
\text { mouse }\end{array}$ & $\begin{array}{l}\text { T cell lymphoma cell line; } \\
\text { hEF1 } 1 / \text { CAG-P-eGFP-Neo }\end{array}$ & (Monecke, 2013) \\
\hline & RMA eGFP-OVA & $\begin{array}{l}\mathrm{C} 57 \mathrm{BL} / 6 \\
\text { mouse }\end{array}$ & $\begin{array}{l}\text { T cell lymphoma cell line; } \\
\text { hEF } 1 \alpha / C A G-P-O V A-e G F P- \\
\text { Neo }^{R}\end{array}$ & (Monecke, 2013) \\
\hline & YAC-1 & $\begin{array}{l}\mathrm{A} / \mathrm{Sn} \\
\text { mouse }\end{array}$ & Lymphoma cell line & \\
\hline
\end{tabular}

OVA-expressing stem cell lines were previously constructed by electrophoretic transfection of an expression construct for OVA-eGFP under the control of the ubiquitously active hEF1 $\alpha$ or CAG promoter and selection for Neomycin resistance (Monecke, 2013). 


\subsubsection{Laboratory animals}

All animal experiments had been approved by the local government and were in accordance with institutional guidelines for the welfare of animals. Laboratory animals were bred in the central animal facility of the University Medical Center (UMC) Göttingen, where they were kept under specific pathogen-free conditions in individually-ventilated cages (IVC) in a 12 hrs light-dark cycle. Female (f) and male $(\mathrm{m})$ mice between 8 and 20 weeks of age were used for experiments.

Table 2 Laboratory animals

\begin{tabular}{|c|c|c|c|}
\hline Strain & Organism & Description & Reference \\
\hline $129 / \mathrm{Sv}$ & mouse & MHC haplotype: $\mathrm{H}^{\mathrm{b}}$ & \\
\hline BUF & rat & MHC haplotype: RT1 ${ }^{\mathrm{b}}$ & \\
\hline cMy-mOVA & mouse & $\begin{array}{l}\text { mice are homozygous for the } \alpha \text { - } \\
\text { MyHC-P-mOVA transgene }\end{array}$ & (Grabie et al., 2003a) \\
\hline cMy-mOVA/OT-I & mouse & $\begin{array}{l}\alpha-M y H C-P-m O V A ; \text { transgenic for a } \\
\text { TCR recognizing SIINFEKL/H2K }\end{array}$ & \\
\hline cMy-mOVA/OT-II & mouse & $\begin{array}{l}\alpha-M y H C-P-m O V A ; \text { transgenic for a } \\
\text { TCR recognizing } \\
\text { ISQAVHAAHAEINEAGR } / H 2^{b}\end{array}$ & \\
\hline $\mathrm{LOU} / \mathrm{c}$ & rat & MHC haplotype: RT1u & \\
\hline$N K G 2 D^{-/-}$ & mouse & $\begin{array}{l}\text { Introduced mutation in exon } 2 \text { of } \\
\text { NKG2D (KIrk1) locus }\end{array}$ & (Zafirova et al., 2009) \\
\hline OT-I & mouse & $\begin{array}{l}\text { transgenic for a TCR recognizing } \\
\text { SIINFEKL/H2K }{ }^{b}\end{array}$ & (Hogquist et al., 1994) \\
\hline OT-II & mouse & $\begin{array}{l}\text { transgenic for a TCR recognizing } \\
\text { ISQAVHAAHAEINEAGR/ H2 }\end{array}$ & (Barnden et al., 1998) \\
\hline $\mathrm{RAG}^{-1-}$ & mouse & $\begin{array}{l}\text { Immunodeficiency affecting B and } \\
T \text { cells }\end{array}$ & (Shinkai et al., 1992) \\
\hline $\mathrm{RAG}^{-1-\gamma \mathrm{C}^{-/-}}$ & mouse & $\begin{array}{l}\text { severe combined } \\
\text { immunodeficiency affecting } B, T \\
\text { and NK cells }\end{array}$ & (Mombaerts et al., 1992) \\
\hline SCID & mouse & $\begin{array}{l}\text { severe combined } \\
\text { immunodeficiency affecting B and } \\
\text { T cells }\end{array}$ & (Pla and Mahouy, 1991) \\
\hline SCID/beige & mouse & $\begin{array}{l}\text { severe combined } \\
\text { immunodeficiency affecting } B, T \\
\text { and NK cells }\end{array}$ & $\begin{array}{l}\text { (Froidevaux and Loor, } \\
\text { 1991) }\end{array}$ \\
\hline
\end{tabular}




\subsubsection{Antibodies}

Antibodies were used according to manufacturer's instructions.

Table 3 Primary murine antibodies.

\begin{tabular}{|c|c|c|c|c|}
\hline Antigen & Isotype & Clone & Label & Supplier \\
\hline CD115 & rat IgG2a & TX56 & Alexa 488 & BioLegend \\
\hline CD11b & rat $\lg G 2 b$ & $\mathrm{M} 1 / 70$ & PE/Cy5 & BioLegend \\
\hline CD11c & hamster IgG & N418 & $\mathrm{PE}$ & BioLegend \\
\hline CD144 & rat IgG1 & BV13 & $A P C$ & BioLegend \\
\hline CD19 & rat IgG2b & PeCa1 & FITC & BioLegend \\
\hline CD25 & rat IgG1 & PC61.5.3 & FITC & BioLegend \\
\hline CD3 & mouse IgG2b & $17 A 2$ & FITC & BioLegend \\
\hline CD3 & mouse IgG $2 b$ & $17 A 2$ & PE & BioLegend \\
\hline CD31 & rat IgG2a & 390 & $\mathrm{PE}$ & BioLegend \\
\hline CD314 (NKG2D) & mouse IgG1 & 149810 & $\mathrm{PE}$ & R\&D Systems \\
\hline CD4 & rat IgG2a & RM4-5 & PE/Cy5 & BioLegend \\
\hline CD45 & rat IgG2b & 30-F11 & FITC, PE, APC & BioLegend \\
\hline CD45.1 & mouse $\operatorname{lgG} 2 a$ & A20 & FITC & BioLegend \\
\hline CD45.2 & mouse IgG2a & 104 & FITC & BioLegend \\
\hline CD45R & rat IgG2a & RA2-6B2 & unconjugated & BioLegend \\
\hline CD45R & rat IgG2a & RA2-6B2 & $\mathrm{PE}$ & BioLegend \\
\hline CD49b (DX5) & rat IgM & DX5 & PE & BioLegend \\
\hline CD69 & hamster IgG & $\mathrm{H} 1.2 \mathrm{~F} 3$ & PE & BioLegend \\
\hline CD8a & rat IgG2a & $53-6.7$ & PE/Cy5 & BioLegend \\
\hline $\mathrm{F} 4 / 80$ & rat IgG2b & A3-1 & unconjugated & BioLegend \\
\hline $\mathrm{F} 4 / 80$ & rat IgG2a & BM8 & PE & BioLegend \\
\hline FoxP3 & mouse IgG1 & 159D & PE & BioLegend \\
\hline $\mathrm{H} 60$ & rat IgG2a & 205326 & unconjugated & RD \\
\hline I-Ab MHC II & mouse IgG2a & AF6-120.1 & FITC & BioLegend \\
\hline IFNp & rat IgG2b & JES5-16E3 & PE & BioLegend \\
\hline IL-10 & rat IgG1 & MP5-20F3 & $\mathrm{PE}$ & BioLegend \\
\hline IL-17A & rat IgG1 & MP6-XT22 & PE & BioLegend \\
\hline IL-17F & rat IgG1 & TC11-18H10.1 & $\mathrm{PE}$ & BioLegend \\
\hline IL-2 & mouse IgG1 & 13/45/31-2 & ohne & Dianova \\
\hline IL-4 & rat IgG2b & JES6-5H4 & $\mathrm{PE}$ & BioLegend \\
\hline IL-6 & rat IgG1 & 11B11 & PE & BioLegend \\
\hline Ki-67 & rat IgG2a & TEC3 & unconjugated & DAKO \\
\hline Ly6C & rat lgG2b & HK1.4 & PE & BioLegend \\
\hline Ly6G & rat IgG2b & RB6-8C5 & PE & BioLegend \\
\hline Mult-1 & rat IgG2a & 237104 & unconjugated & RD \\
\hline NKp46 & rat IgG2a & 29A1.4 & unconjugated & BioLegend \\
\hline NKp46 & rat IgG2a & 29A1.4 & PE & BioLegend \\
\hline Oct3/4 & mouse IgG1 & 40-Oct3 & unconjugated & BioLegend \\
\hline OVA ascites & mouse IgG1 & OVA-14 & unconjugated & Sigma \\
\hline
\end{tabular}




\begin{tabular}{|l|l|l|l|l|}
\hline RAE-1 (pan) & rat IgG2a & 186107 & unconjugated & RD \\
\hline TCR V $\beta 5.1,5.2$ & mouse IgG1 & MR9-4 & FITC & BD \\
\hline TGFß1 & mouse IgG1 & 9D3.1C8 & PE & BioLegend \\
\hline TNF $\alpha$ & rat IgG1 & XMG1.2 & PE & BioLegend \\
\hline Y $\delta$ TCR & hamster IgG & GL3 & PE & BioLegend \\
\hline
\end{tabular}

Table 4 Secondary antibodies and ELISA antibodies

\begin{tabular}{|l|l|l|l|l|}
\hline Antigen & Isotype & Clone & Label & Supplier \\
\hline anti-mouse APC & goat IgG & Poly4053 & & BioLegend \\
\hline anti-mouse Cy3 & $\begin{array}{l}\text { donkey IgG } \\
\text { F(ab')2-fragment }\end{array}$ & & & $\begin{array}{l}\text { Jackson } \\
\text { ImmunoResearch }\end{array}$ \\
\hline anti-mouse FITC & goat IgG & Poly4053 & FITC & BioLegend \\
\hline anti-mouse IgA & rat IgG1 & C10-1 & & BD \\
\hline anti-mouse IgE & rat IgG1 & R35-72 & & BD \\
\hline anti-mouse IgG & goat IgG1 & M35006 & TC & BioLegend \\
\hline anti-mouse IgG1 & rat IgG1 & A85-1 & & BD \\
\hline anti-mouse IgG2a & rat IgG1 & R11-89 & & BD \\
\hline anti-mouse IgG2b & rat IgG2a & R12-3 & & BD \\
\hline anti-mouse IgG3 & rat IgG1 & R2-38 & & BD \\
\hline anti-mouse IgM & rat IgG2a & II-41 & & BD \\
\hline
\end{tabular}

Table 5 Isotype controls

\begin{tabular}{|l|l|l|}
\hline Isotype & Label & Supplier \\
\hline hamster IgG & PE & BioLegend \\
\hline mouse IgG1 & FITC & BioLegend \\
\hline mouse IgG1 & PE & Immunotools \\
\hline mouse IgG2a & FITC & Pharmingen \\
\hline mouse IgG2b & PE & BioLegend \\
\hline mouse IgG2b & FITC & BioLegend \\
\hline mouse IgM & FITC & BioLegend \\
\hline rat IgG1 & FITC & BioLegend \\
\hline rat IgG1 & PE & BioLegend \\
\hline rat IgG1 & APC & BioLegend \\
\hline rat IgG2a & FITC & BioLegend \\
\hline rat IgG2a & PE & BioLegend \\
\hline rat IgG2a & PE/Cy5 & BioLegend \\
\hline rat IgG2a & Alexa 488 & BioLegend \\
\hline rat IgG2b & FITC & BioLegend \\
\hline rat IgG2b & PE & BioLegend \\
\hline rat IgG2b & APC & BioLegend \\
\hline rat IgM & FITC & BioLegend \\
\hline rat IgM & PE & BioLegend \\
\hline rat IgM & PE/Cy5 & BioLegend \\
\hline & & \\
\hline
\end{tabular}




\subsubsection{Enzymes}

Table 6 Enzymes

\begin{tabular}{|c|c|}
\hline Enzyme & Supplier \\
\hline DNase I & NEB \\
\hline Liberase & Roche \\
\hline M-MLV Reverse Transcriptase & Promega \\
\hline Proteinase $\mathrm{K}$ & Merck \\
\hline RNAse A & Roche Diagnostics \\
\hline RNAsin $^{\circledast}$ & Promega \\
\hline Taq DNA Polymerase & NEB \\
\hline
\end{tabular}

\subsubsection{Chemicals and Reagents}

Table 7 Chemicals and reagents

\begin{tabular}{|l|l|}
\hline Chemical/Reagent & Supplier \\
\hline 2,2'-azino-bis(3-ethylbenzothiazoline-6-sulphonic acid (ABTS) & AppliChem \\
\hline 2- $\beta$-Mercaptoethanol & SIGMA-Aldrich ${ }^{\circledR}$ \\
\hline 3,3'-diaminobenzidine (DAB) & Carl Roth \\
\hline ABsolute Blue qPCR SYBR Green low Rox Mix & Thermo Scientific \\
\hline ABTS $^{\circledR}$ ELISA HRP Substrate & KPL, Sera Care \\
\hline Acetic acid & Merck \\
\hline Ammonium chloride (NH ${ }_{4}$ Cl) & Merck \\
\hline Bovine Serum Albumin (BSA) 100x & Promega \\
\hline Carboxyflourescein diacetate succinimidyl ester (CFSE) & Life Technologies \\
\hline Chloroform & Merck \\
\hline Dimethyl sulfoxide (DMSO) & Merck \\
\hline Direct Red 80 & Sigma \\
\hline DL-Dithiothreitol (DTT) & Promega \\
\hline dNTPs & NEB \\
\hline Eosin Y & Merck Millipore \\
\hline ESGRO ${ }^{\circledR}$ Leukemia Inhibory Facator (LIF) & Merck Millipore \\
\hline Ethanol (analytical grade) & UMG Apotheke \\
\hline Ethanol (EtOH) & UMG Apotheke \\
\hline Ethidium bromide & HyClon \\
\hline Ethylenediaminetetraacetic acid (EDTA) & Carl Roth \\
\hline FACSflow & BD Biosciences \\
\hline FACS ${ }^{\text {TM }}$ Lysis solution & BD Biosciences \\
\hline Fetal calf serum (FCS) & Biochrom \\
\hline Forene (Isofluran) & Abbvie \\
\hline Geltrex (Growth Factor Reduced) & Life Technologies \\
\hline GeneRuler 1 kb DNA ladder & Thermo Scientific \\
\hline Geneticin (G418 Sulfate) & Biochrom \\
\hline GIBCO ${ }^{\circledR}$ Penicillin/Streptomycin & Merck \\
\hline & \\
\hline
\end{tabular}




\begin{tabular}{|c|c|}
\hline Heparin sodium & Rotexmedica \\
\hline Hydrochloric acid $(\mathrm{HCl})$ & Merck \\
\hline Hydrogen peroxide $\left(\mathrm{H}_{2} \mathrm{O}_{2}\right)$ & Merck \\
\hline Isoamylalcohol & Merck \\
\hline Isopropanol & Merck \\
\hline Magnesium chloride $\left(\mathrm{MgCl}_{2}\right)$ & Merck \\
\hline Mayer's hemalum solution & Merck Millipore \\
\hline Methanol & Merck \\
\hline Mitomycin C & AppliChem \\
\hline MLV-RT Puffer & Promega \\
\hline M-MLV Puffer & Promega \\
\hline Non-essential amino acids & Merk Millipore \\
\hline Novaminsulfon (Metamizol) & Zentiva \\
\hline Ovalbumin (OVA) & Sigma \\
\hline Paraffin & Carl Roth \\
\hline PCR-Puffer 10x & NEB \\
\hline Picric acid & Morphisto \\
\hline Potassium chloride (KCl) & Carl Roth \\
\hline Potassium phosphate $\left(\mathrm{KH}_{2} \mathrm{PO}_{4}\right)$ & Merck \\
\hline Pre-stained protein marker, broad range & NEB \\
\hline Propidium iodide (PI) & AppliChem \\
\hline Random primer & Promega \\
\hline Recombinant mouse interleukin-2 (IL-2) & $R$ \& D Systems \\
\hline RNasin ${ }^{\circledR}$ Plus Ribonuclease Inhibitor & Promega \\
\hline Roswell Park Memorial Institute medium (RPMI) & Gibco \\
\hline Roti $^{\circledR}$-Histokitt & Carl Roth \\
\hline Roti $^{\circledR}$-Phenol & Carl Roth \\
\hline Scintillator Optiphase HiSafe 3 & PerkinElmer \\
\hline SIINFEKL (Ovalbumin amino acid (aa) 257-264) & Bachem AG \\
\hline Sodium carbonate $\left(\mathrm{Na}_{2} \mathrm{CO}_{3} / \mathrm{NaHCO}_{3}\right)$ & Merck \\
\hline Sodium chloride $(\mathrm{NaCl})$ & Carl Roth \\
\hline Sodium chromate $\left(\mathrm{Na}_{2}{ }^{51} \mathrm{CrO}_{4}\right)$ & Hartmann Analytic \\
\hline Sodium hydroxide $(\mathrm{NaOH})$ & Carl Roth \\
\hline Sodium phosphate $\left(\mathrm{Na}_{2} \mathrm{HPO}_{4} / \mathrm{NaH}_{2} \mathrm{PO}_{4}\right)$ & Carl Roth \\
\hline Streptavidin-horseradish peroxidase (HRP) & BioLegend \\
\hline Sucrose & Carl Roth \\
\hline TiterMax $^{\circledR}$ & TiterMax \\
\hline Tris & Carl Roth \\
\hline Triton X-100 & AppliChem \\
\hline TRIzol ${ }^{\circledR}$ Reagent & Invitrogen ${ }^{\mathrm{TM}}$ \\
\hline Trypan blue & SIGMA-Aldrich $^{\circledR}$ \\
\hline Trypsin & Biochrom \\
\hline UltraPure ${ }^{\mathrm{TM}}$ Agarose & Invitrogen \\
\hline VLE Dulbecco's Modified Eagle's medium (DMEM) & Gibco \\
\hline Xylol & Carl Roth \\
\hline
\end{tabular}




\subsubsection{Disposables}

Table 8 Disposables

\begin{tabular}{|c|c|}
\hline Label & Supplier \\
\hline 96 - well plates for qPCR & Applied Biosystems \\
\hline 96 - well Wallac plates & PerkinElmer \\
\hline Cell culture 96 - and 24-well plates & Sarstedt \\
\hline Cell culture flasks T25 & Sarstedt \\
\hline Cell culture plates $(5 \mathrm{ml}, 10 \mathrm{ml}, 25 \mathrm{ml})$ & Greiner \\
\hline Cell culture plates $(6,12,24$ well) & Greiner/Thermo Scientific \\
\hline Cell culture plates 96 well round bottom & Sarstedt \\
\hline Cell strainer & Falcon \\
\hline Conical tubes (13 ml, $15 \mathrm{ml}, 50 \mathrm{ml})$ & Greiner/Sarstedt \\
\hline Cover slips glass & Roth \\
\hline Cover slips plastic & Sarstedt \\
\hline Cryo tubes & Greiner \\
\hline FACS tubes & BD/Sarstedt \\
\hline MACS LS columns & MACS Miltenyi Biotec \\
\hline Microscope slides & Menzel \\
\hline Multipette ${ }^{\circledR}$ plus Combitips & Eppendorf \\
\hline Needles 21 G x 1 1/2", 22 G x 1 1/4", 26 G x 1/2", 30 G x 1/2" & B. Braun Melsungen AG \\
\hline Nunc MaxiSorp microtiter plates & Thermo Scientific \\
\hline Parafilm & Pechiney Plastic Packaging \\
\hline Pasteur pipettes & Wilhelm Ulbrich Mainz \\
\hline Pipette Tips $(10 \mu \mathrm{l}, 20 \mu \mathrm{l}, 200 \mu \mathrm{l}, 1000 \mu \mathrm{l})$ & Greiner/Sarstedt \\
\hline Reaction Tubes $(0.2 \mathrm{ml}, 1 \mathrm{ml}, 2 \mathrm{ml})$ & Greiner/Sarstedt \\
\hline Sterile filters (pore size $0.2 \mu \mathrm{m}, 0.45 \mu \mathrm{m}$ ) & Greiner \\
\hline Sterile pipettes $(1 \mathrm{ml}, 2 \mathrm{ml}, 5 \mathrm{ml}, 10 \mathrm{ml}, 25 \mathrm{ml})$ & Greiner \\
\hline Superfrost Plus glass slides & Thermo \\
\hline Syringes $(1 \mathrm{ml}, 2 \mathrm{ml}, 5 \mathrm{ml}, 10 \mathrm{ml}, 20 \mathrm{ml})$ & Becton Dickinson \\
\hline Top Seal for qPCR plates & Applied Biosystems \\
\hline Top seal for Wallac plates & PerkinElmer \\
\hline U-100 Insulin syringes $0.5 \mathrm{ml}$ BD Micro-Fine ${ }^{\mathrm{TM}}$ & $\mathrm{BD}$ \\
\hline Wheighing paper & Machery-Nagel \\
\hline
\end{tabular}




\subsubsection{Devices}

Table 9 Devices

\begin{tabular}{|c|c|c|}
\hline Description & Label & Supplier \\
\hline $\begin{array}{l}\text { Agarose gel trays and } \\
\text { chambers }\end{array}$ & Perfect Blue ${ }^{\mathrm{TM}}$ Gel System & Peglab \\
\hline Aqua bidest. Supply & arium $^{\circledR}$ pro & Sartorius \\
\hline Autoclave & High pressure steam sterilisator FVS & Integra Biosciences \\
\hline Biological Safety Cabinet & HERASave $^{\circledR}$ & Thermo Fisher Scientific \\
\hline Centrifuge & Multifuge $3 \mathrm{~S}-\mathrm{R}$ & Heraeus \\
\hline Centrifuge & Mini Centrifuge MCF-2360 & Heraeus \\
\hline Centrifuge & $3 \mathrm{~K} 30$ & LMS Consult \\
\hline Centrifuge & RC 3B Plus & Sigma \\
\hline Centrifuge & Mini Centrifuge MCF-2360 & Sorvall \\
\hline Counting chamber & Neubauer improved & Krannich \\
\hline Dispenser & Multipette ${ }^{\circledast}$ plus & Eppendorf \\
\hline $\begin{array}{l}\text { Echocardiography digital } \\
\text { imaging platform }\end{array}$ & Vevo2100 & $\begin{array}{l}\text { VisualSonics, Toronto, } \\
\text { Canada }\end{array}$ \\
\hline ELISA reader & $\begin{array}{l}\text { PowerWave } 340 \text { microplate } \\
\text { spectrophotometer }\end{array}$ & BioTek \\
\hline Flow Cytometer & FACS Calibur & Becton Dickinson \\
\hline Freezer & HERA freeze $-80^{\circ} \mathrm{C}$ & Heraeus \\
\hline Freezer & Liebherr Comfort $-20^{\circ} \mathrm{C}$ & Liebherr GmbH \\
\hline Freezer & VIP plus $-150^{\circ} \mathrm{C}$ & SANYO Electric Co., Japan \\
\hline Homogenizer & Tenbroeck & schuett-biotec \\
\hline Imaging devices & Chemilux Blot Detection Imager & Intas \\
\hline Imaging devices & UV workbench Gellmager & Intas \\
\hline Incubator shaker & Unitron-plus & Infors \\
\hline Incubators & HERACell 150 & Heraeus \\
\hline Liquid Scintillation Counters & MicroBeta2 Plate Counter & PerkinEImer \\
\hline MACS separator & VarioMACS ${ }^{\mathrm{M}}$ Separator & MACS Miltenyi Biotec \\
\hline Magnetic stirrer/heater & RH basic 2 & IKA \\
\hline Microscope & Axiovert 35 & Zeiss \\
\hline Microscope & LSM 510 Axioplan 2 & Zeiss \\
\hline Microtome & Leica RM2255 & Leica Biosystems \\
\hline Microwave & HF12M 900W & Siemens \\
\hline pH-Meter & inoLab $^{\circledR}$ pH Level 1 & WTW \\
\hline Pipettes & Research $^{\circledast}$ \& Reference ${ }^{\circledR}$ & Eppendorf \\
\hline Pipettor & IBS PIPETBOY acu & Integra Bioscience \\
\hline Power supply & EPS-301/-3501 XL & GE Heathcare \\
\hline Scales & ACCULAB Vicon & Sartorius \\
\hline Scales & BP 61 & Sartorius \\
\hline Slide scanner & dotSlide - Virtual slide system & Olympus Life Science \\
\hline Spectrophotometer & NanoDrop ${ }^{\mathrm{TM}}$ ND-1000 & Thermo Fisher Scientific \\
\hline Thermal Cycler & ABI 7500 Real-Time PCR System & Applied Biosystems \\
\hline Thermal Cycler & MasterCycler epgradient & Eppendorf \\
\hline
\end{tabular}




\begin{tabular}{|l|l|l|}
\hline Thermal Cycler & TPersonal 48 & Biometra \\
\hline Thermoblock & Thermomixer comfort & Eppendorf \\
\hline Thermoblock & Stuart Block Heater SBH130 & Bibby Scientific \\
\hline Vortexer & MS1 Minishaker & IKA \\
\hline Water bath & Medingen W6 & Labortechnik Medingen \\
\hline
\end{tabular}

\subsubsection{Commercial kits}

Table 10 Commercial kits

\begin{tabular}{|l|l|}
\hline Label & Supplier \\
\hline ABsolute $^{\mathrm{TM}}$ Blue QPCR SYBR \\
\hline Cytofix $^{\circledR}$ Gytoperm ${ }^{\mathrm{TM}}$ Kit & Thermo Fisher \\
\hline GeneJET Genomic DNA Purification Kit & BD \\
\hline Mouse CD4 ${ }^{+}$T cell isolation kit (MACS) & Thermo Scientific \\
\hline Mouse CD8a ${ }^{+}$T cell isolation kit (MACS) & Miltenyi Biotec \\
\hline Mouse NK cell isolation kit (MACS) & Miltenyi Biotec \\
\hline
\end{tabular}

\subsubsection{Buffers and Solutions}

The composition of used buffers and solutions is specified in the corresponding method section. Solutions were autoclaved at $125^{\circ} \mathrm{C}$ for $30 \mathrm{~min}$ or filtrated with a $0.2 \mu \mathrm{m}$ sterile filter for sterilization. A consistently used buffer was PBS with the following composition:

$\begin{array}{lll}\text { PBS } & 137 \mathrm{mM} & \mathrm{NaCl} \\ 2.7 \mathrm{mM} & \mathrm{KCL} \\ 10 \mu \mathrm{M} & \mathrm{Na}_{2} \mathrm{HPO}_{4} \\ & 2.0 \mathrm{mM} & \mathrm{KH}_{2} \mathrm{PO}_{4} \\ \text { dissolved in } & \mathrm{dH}_{2} \mathrm{O}, \mathrm{pH} 7.4\end{array}$

\subsubsection{Oligonucleotides}

All primers were synthesized by biomers.net $\mathrm{GmbH}$. The qPCR primers were designed using IDT's PrimerQuest that incorporates Primer3 software developed by the Whitehead Institute for Biomedical Research. The specificity of the primers was verified with the nucleotide BLAST web tool from NCBI. The primers were designed to target all transcript variants if several isoforms exist and to span exonexon junctions if feasible. 
Table 11 Synthetic oligonucleotides used for qPCR. Mus musculus. Dissociation temperature [ $\left.{ }^{\circ} \mathrm{C}\right]$, P.E.: Primer efficiencies, *(Monecke, 2013)

\begin{tabular}{|c|c|c|c|c|c|c|c|}
\hline & Gene & & Sequence & RefSeq & ${ }^{\circ} \mathrm{C}$ & P.E & $*$ \\
\hline \multirow{6}{*}{ 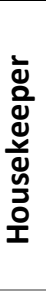 } & \multirow[t]{2}{*}{ Gapdh } & $\mathrm{F}$ & TGTGTCCGTCGTGGATCTGA & \multirow{2}{*}{$\begin{array}{l}\text { RTPrimerDB ID: } \\
7880\end{array}$} & \multirow[t]{2}{*}{85} & \multirow[t]{2}{*}{2.0} & \multirow[t]{2}{*}{ * } \\
\hline & & $\mathrm{R}$ & TTGCTGTTGAAGTCGCAGGAG & & & & \\
\hline & \multirow[t]{2}{*}{ Hprt } & $\mathrm{F}$ & GTCCTGTGGCCATCTGCCTA & \multirow[t]{2}{*}{ NM_013556.2 } & \multirow[t]{2}{*}{78} & \multirow[t]{2}{*}{1.9} & \multirow[t]{2}{*}{ * } \\
\hline & & $\mathrm{R}$ & GGGACGCAGCAACTGACATT & & & & \\
\hline & \multirow[t]{2}{*}{$U b c$} & $\mathrm{~F}$ & AGGTCAAACAGGAAGACAGACGTA & \multirow[t]{2}{*}{ RTPrimerDB ID: 42} & \multirow[t]{2}{*}{79} & \multirow[t]{2}{*}{1.8} & \multirow[t]{2}{*}{$*$} \\
\hline & & $\mathrm{R}$ & TCACACCCAAGAACAAGCACA & & & & \\
\hline \multirow{4}{*}{ 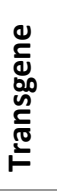 } & \multirow[t]{2}{*}{ Ova } & $\mathrm{F}$ & AGAGGTGGTAGGGTCAGCAGAGG & \multirow[t]{2}{*}{ NM_205152.1 } & \multirow[t]{2}{*}{82} & \multirow[t]{2}{*}{1.9} & \multirow[t]{2}{*}{$*$} \\
\hline & & $\mathrm{R}$ & TGGTTGCGATGTGCTTGATACAGAAG & & & & \\
\hline & \multirow[t]{2}{*}{ Egfp } & $\mathrm{F}$ & CAAGCAGAAGAACGGCATCAAGGT & \multirow[t]{2}{*}{ U55761.1 } & \multirow[t]{2}{*}{88} & \multirow[t]{2}{*}{1.9} & \multirow[t]{2}{*}{$*$} \\
\hline & & $\mathrm{R}$ & ACTGGGTGCTCAGGTAGTGGTT & & & & \\
\hline ఏे & Nanog & $\mathrm{F}$ & GCTGATTTGGTTGGTGTCTTGCTC & NM_028016.3 & 80 & 1.9 & \\
\hline 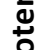 & & $\mathrm{R}$ & TGTGATGGCGAGGGAAGGGATTT & & & & \\
\hline 을 & Oct4 & $\mathrm{F}$ & ATTGAGAACCGTGTGAGGTGGA & NM_013633.3 & 81 & 2.0 & \\
\hline & & $\mathrm{R}$ & GATGTGAGTGATCTGCTGTAGGGA & & & & \\
\hline & Сурза11 & $\mathrm{F}$ & ACAAGCAGGGATGGACCTGG & NM_007818.3 & 82 & 2.0 & \\
\hline & & $\mathrm{R}$ & TGTGACAGCAAGGAGAGGCG & & & & \\
\hline б & Hormad1 & $\mathrm{F}$ & TGTTTGTCACCTACACTCAGG & NM_026489.2 & 80 & 2.0 & \\
\hline$\frac{\mathrm{t}}{\frac{\pi}{3}}$ & & $\mathrm{R}$ & GTAAGGAAGAAGAAACTATGC & & & & \\
\hline$\overline{0}$ & $\mathrm{Zg16}$ & $\mathrm{F}$ & TGATCTTCGTGACGGACAAG & NM_026918.2 & 81 & 2.0 & \\
\hline$\underline{\sigma}$ & & $\mathrm{R}$ & CCAGATCGCCCACTAATGAA & & & & \\
\hline 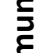 & Ido & $\mathrm{F}$ & CCACACTGAGCACGGACGG & NM_008324.1 & 84 & 2.0 & * \\
\hline$\underline{\bar{\xi}}$ & & $\mathrm{R}$ & TGCGGGGCAGCACCTTTCG & & & & \\
\hline & $\operatorname{Arg} 1$ & $\mathrm{~F}$ & ACCTGGCCTTTGTTGATGTCC & NM_007482.3 & 83 & 1.8 & * \\
\hline & & $\mathrm{R}$ & AGCACCACACTGACTCTTCСАTTC & & & & \\
\hline & $\alpha-M h c$ & $\mathrm{~F}$ & GCAGGAGCTGATTGAGACCA & NM_010856.4 & 85 & & \\
\hline & & $\mathrm{R}$ & CTGTCTGGAGTTGGGTCAGG & & & & \\
\hline & Th & $\mathrm{F}$ & GTCTCAGAGCAGGATACCAAGC & NM_009377 & 89 & & \\
\hline & & $\mathrm{R}$ & СTCTCCTCGAATACCACAGCC & & & & \\
\hline & Syn & $\mathrm{F}$ & CAGTTCCGGGTGGTCAAGG & NM_009305 & 86 & & \\
\hline$\underset{\frac{\pi}{\pi}}{\pi}$ & & $\mathrm{R}$ & ACTCTCCGTCTTGTTGGCAC & & & & \\
\hline$\underline{\xi}$ & Ctnt & $\mathrm{F}$ & AGAGGACACCAAACCCAAGC & NM_001130176.1 & 85 & & \\
\hline ธ & & $\mathrm{R}$ & CCTGTGGATGTCATCAAAGTCC & & & & \\
\hline & $A l b$ & $\mathrm{~F}$ & GGCGACTATCTCCAGCAAACT & NM_009654.3 & 83 & & \\
\hline & & $\mathrm{R}$ & TCATACAAGAACGTGCCCAGG & & & & \\
\hline & Afp & $\mathrm{F}$ & AGTGCGTGACGGAGAAGAAT & NM_007423 & 83 & & \\
\hline & & $R$ & AAACACCCATCGCCAGAGTT & & & & \\
\hline
\end{tabular}




\subsubsection{Software and Databases}

Table 12 Software and databases

\begin{tabular}{|l|l|}
\hline Product & Company \\
\hline cellSens dimension & Olympus Life Science \\
\hline 7000 System SDS Software & Applied Biosystems \\
\hline CellQuest Pro & BD Biosciences \\
\hline FlowJo & Tree Star, Inc., USA \\
\hline MFE primer-2.0 & GitHub Inc. \\
\hline MicroBeta Workstation & PerkinElmer \\
\hline Microsoft Office Professional Plus 2013 & Microsoft \\
\hline NCBI database & National Center for Biotechnology Information (NCBI) \\
\hline Oligo Analyzer & Integrated DNA Technologies (IDT) \\
\hline Primer Quest & Integrated DNA Technologies (IDT) \\
\hline qPCR primer database & RTprimerDB.org \\
\hline WinStat & R. Fitch Software \\
\hline Zotero Standalone for Windows & Roy Rosenzweig Center for History and New Media \\
\hline
\end{tabular}




\subsection{Methods}

\subsubsection{Biomolecular methods}

\subsubsection{Isolation of nucleic acids}

\subsection{Genomic DNA preparation}

DNA was prepared from cell pellets $\left(1 \times 10^{6}\right.$ to $5 \times 10^{6}$ cells) using the GeneJET Genomic DNA Purification Kit according to manufacturer's instructions. DNA was dissolved in $\mathrm{dH}_{2} \mathrm{O}$ and stored at $4{ }^{\circ} \mathrm{C}$. The quantity of DNA was determined using the Nanodrop 2000 . Thereby, the ratio of $260 \mathrm{~nm}$ to $280 \mathrm{~nm}$ values was an important criterion for sample purity (pure DNA: ratio $\geq 1.8$ ).

\subsection{RNA preparation}

RNA was isolated from $1 \times 10^{6}$ to $5 \times 10^{6}$ cells or $100 \mathrm{mg}$ tissue using TRIzol ${ }^{\circledR}$ reagent according to the manufacturer's protocol. To eliminate trace amounts of DNA, RNA samples were digested with DNAse I for $30 \mathrm{~min}$ at $37^{\circ} \mathrm{C}$ using the following reagents:

$\begin{array}{ll}5 \% & 1 \mathrm{M}_{\text {Tris}}-\mathrm{HCl} \mathrm{pH} 7.5 \\ 1 \% & \mathrm{MgCl}_{2} \\ 1 \% & \text { DNAse I }(10 \mathrm{U} / \mu \mathrm{l}) \\ 0.5 \% & \text { RNAse Inhibitor }(40 \mathrm{U} / \mu \mathrm{l}) \\ 50 \% & \text { total RNA eluate } \\ \text { add to } 50 \mu \mathrm{l} & \mathrm{dH}_{2} \mathrm{O}\end{array}$

After a phenol chlorophorm extraction and alcohol precipitation (Ch. 3.2.1.2.2), the RNA was dissolved in an appropriate amount of RNase-free $\mathrm{dH}_{2} \mathrm{O}$ and stored at $-80^{\circ} \mathrm{C}$. The quality of RNA was assured by visualization of the integrity of $18 \mathrm{~S}$ and $28 \mathrm{~S}$ RNA loaded on a $1 \%$ agarose gel, while the quantity was determined using the Nanodrop 2000 at a wavelength of $260 \mathrm{~nm}$.

\subsubsection{Purification of nucleic acids}

\subsection{Purification of nucleic acids via alcohol precipitation}

Nucleic acids were precipitated from aqueous solution by adding $1 / 10$ volume of $3 \mathrm{M}$ sodium acetate $(\mathrm{pH} 4.8 ; 0.3 \mathrm{M}$ final) and one volume isopropanol or 2.5 volumes pure ethanol, respectively. Alternatively, $1 / 3$ volume of $7.5 \mathrm{M}(2.5 \mathrm{M}$ final) ammonium acetate was used instead of sodium acetate to avoid coprecipitation of short oligonucleotides. Samples were centrifuged (15000 rpm; 
$4{ }^{\circ} \mathrm{C}$; $30 \mathrm{~min}$ ) and the resulting DNA pellet was washed with $70 \%$ ethanol before solubilization in an appropriate volume of $\mathrm{dH}_{2} \mathrm{O}$. DNA was stored at $-20^{\circ} \mathrm{C}$.

\subsection{Precipitation of nucleic acids via phenol-chloroform extraction}

Proteins and other contaminants were removed from nucleic acid solutions using phenol chloroform extraction. For this, an equal volume of phenol-chloroform-isoamylalcohol (25:24:1) was added and after thoroughly mixing the solution was centrifuged to gain phase separation. The DNA-containing aqueous phase was transferred into a new reaction tube. The procedure was repeated with 1 volume of chloroform to remove remaining phenol. The nucleic acids were subsequently precipitated by alcohol precipitation.

\subsubsection{Amplification of nucleic acids}

\subsection{Polymerase chain reaction}

The polymerase chain reaction (PCR) is a common used in vitro technique to amplify a specific DNA segment. Main features are the three distinct temperature steps allowing denaturation of doublestranded DNA, primer annealing and elongation by a DNA polymerase. For analytical purposes, Taq polymerase was used and a reaction was set up as follows:

$\begin{array}{ll}2.5 \mu \mathrm{l} & 10 \times \text { Taq PCR buffer } \\ 0.5 \mu \mathrm{l} & \text { dNTP-Mix (10 mM) } \\ 5 \mathrm{pmol} & \text { sense primer } \\ 5 \mathrm{pmol} & \text { antisense primer } \\ 100 \mathrm{ng} & \text { template DNA } \\ 5 \mathrm{U} & \text { Taq polymerase } \\ \text { add to } 25 \mu \mathrm{l} & \mathrm{dH}_{2} \mathrm{O}\end{array}$

The PCR cycler program was adapted with regard to temperature and time for denaturation, annealing and elongation steps. The denaturation and elongation times are associated with the polymerase specifications and were set according to manufacturer's instructions, whereas annealing temperatures were set according to the specific melting temperatures of the primers that were used. 


\subsubsection{2 cDNA synthesis}

RNA samples need to be completely transcribed into complementary DNA (cDNA) for qPCR. Thus, random hexamers, which are short oligodeoxyribonucleotides of random sequence $\left[d(N)_{6}\right]$ that anneal to random complementary sites on the target RNA, and the M-MLV reverse transcriptase were used. A mixture of $2 \mu \mathrm{g}$ RNA and $2 \mu \mathrm{l}$ random hexamer primers was filled with $\mathrm{dH}_{2} \mathrm{O}$ to a final volume of $15 \mu \mathrm{l}$ and was incubated at $70^{\circ} \mathrm{C}$ for $10 \mathrm{~min}$ to break up secondary structures of the RNA. The sample was chilled down on ice to prevent reforming of these structures and subsequently, the following master mix was added:

$5 \mu \mathrm{l} \quad 5 \mathrm{X}$ reverse transcriptase buffer

$2 \mu \mathrm{l} \quad \mathrm{dNTP} \operatorname{mix}(10 \mathrm{mM})$

$1 \mu \mathrm{l} \quad$ DTT $(0.1 \mathrm{M})$

$1 \mu \mathrm{l} \quad$ RNasin plus RNase inhibitor $(40 \mathrm{U} / \mu \mathrm{l})$

$1 \mu \mathrm{l} \quad$ MMLV-reverse transcriptase $(200 \mathrm{U} / \mu \mathrm{l})$

The reverse transcription reaction was incubated at $37^{\circ} \mathrm{C}$ for $1 \mathrm{~h}$ for cDNA synthesis and afterwards stored at $-20^{\circ} \mathrm{C}$.

\subsection{Quantitative real-time PCR}

Quantitative real-time PCR ( $q P C R$ ) was performed to determine the relative amount of specific mRNA transcripts of a gene of interest using the ABI 7500 Real-Time PCR system and the ABsolute ${ }^{\mathrm{TM}}$ Blue QPCR SYBR ${ }^{\circledR}$ green mix. The $\mathrm{CDNA}$ was diluted 1:40 with $\mathrm{dH}_{2} \mathrm{O}$ prior to the reaction, which was set up as follows:

$10 \mu \mathrm{l} \quad$ ABsolute ${ }^{\mathrm{TM}}$ Blue QPCR SYBR ${ }^{\circledR}$ Green Mix

$5 \mathrm{pmol} \quad$ sense primer

$5 \mathrm{pmol} \quad$ antisense primer

$1 \mu \mathrm{l} \quad 1: 40$ template cDNA

add to $20 \mu \mathrm{l} \quad \mathrm{dH}_{2} \mathrm{O}$

Tested genes were analyzed in triplicates to obtain more valid results. Reactions were set up in 96 well plates, which were centrifuged briefly before amplification applying the following PCR program: 


\begin{tabular}{|l|l|l|l|}
\hline PCR step & Temperature & Time & Repeat \\
\hline Enzyme activation & $50^{\circ} \mathrm{C}$ & $2 \mathrm{~min}$ & \\
\hline Initial denaturation & $95^{\circ} \mathrm{C}$ & $10 \mathrm{~min}$ & \\
\hline Denaturation & $95^{\circ} \mathrm{C}$ & $15 \mathrm{sec}$ & \multirow{2}{*}{\} 40 cycles } \\
\hline Annealing/ elongation & $60^{\circ} \mathrm{C}$ & $60 \mathrm{sec}$ & \\
\hline Dissociation stage & $60-90^{\circ} \mathrm{C}$ & $+1{ }^{\circ} \mathrm{C}$ per min & \\
\hline
\end{tabular}

Since SYBR green dye intercalates non-specifically into DNA, unintended products like primer dimers or contaminations could cause a false positive fluorescence signals. Therefore, a stepwise increase of temperature during the dissociation step was included to verify the specificity of the arising products by comparing temperature peaks of the dissociations curves to the known melting temperatures of the intended products. The emerging $\mathrm{PPCR}$ data (cycle threshold $(\mathrm{Ct})$-values) was normalized to a reference gene, i.e. housekepper gene, leading to the $\Delta \mathrm{Ct}$-values. Ct-values over 35 were considered as unspecific due to the late amplification and were excluded from analysis. Gene expression in reference cell lines was set 1 to calculate relative expression of target tissues ( $<1$ : lower expression than reference; > 1: higher expression than reference). The relative quantity of expression was calculated as $2^{-\Delta \Delta C t}$ to the control (Livak and Schmittgen, 2001) or with the specific primer pair efficiency. The primer pair efficiencies were inferred from the following formula: Efficiency $=10^{-1 / \text { slope }}$. Hereby, the slope was calculated from a linear regression based on the obtained Ct-values, when a dilution series of pooled cDNA samples were used as template for each specific primer pair.

\subsection{Housekeeper}

The most stable housekeeping gene for the used cell lines and tissues had to be determined. For this, The three different housekeeping genes Hprt, Gapdh and Ubc were compared in several teratomas to calculate their gene expression stability. The formulas for housekeeper calculation were described in (Vandesompele et al., 2002). Hprt was the most stable housekeeper gene (gene stability measure M $\left.=-3.1 \times 10^{-3}\right)$, followed by Gapdh $(\mathrm{M}=0.014)$ and Ubc $(\mathrm{M}=0.016)$. Consequently Hprt was used as main housekeeper gene for normalization of gene expression and only in experiments in which Gapdh was additionally analyzed, the geometric mean of Hprt and Gapdh was used for normalization (indicated in graphs). 


\subsubsection{Agarose gel electrophoresis}

DNA or RNA fragments were identified and separated by using the Perfect Blue ${ }^{\mathrm{TM}}$ Gel System. The agarose concentration varied between $0.7 \%$ up to $2 \%(\mathrm{w} / \mathrm{v})$ according to the size of the fragments. TAE buffer was used as running buffer and for agarose solutions (dissolved at $100{ }^{\circ} \mathrm{C}$ ). In addition, ethidium bromide was added in concentrations of $0.1 \%(\mathrm{v} / \mathrm{v})$ to identify DNA fragments when exposed to UV light. The DNA samples were supplemented with $6 \times$ DNA loading dye to facilitate the loading procedure and for visualization of the progression during the run. The DNA molecular weight standard GeneRuler $1 \mathrm{~kb}$ DNA ladder was loaded on the gel to determine the size of amplified products. An electric field of 90 to $120 \mathrm{~V}$ and $220 \mathrm{~mA}$ was applied for 10 to $15 \mathrm{~min}$ for RNA and 30 to $60 \mathrm{~min}$ for DNA according to the fragment length. The running time for RNA samples should not exceed 15 min to avoid RNA degradation.

$\begin{array}{lll}6 \text { x loading dye } & 34 \%(\mathrm{w} / \mathrm{v}) & \text { sucrose } \\ & 0.02 \%(\mathrm{w} / \mathrm{v}) & \text { cresol red dye } \\ & \text { dissolved in } & \mathrm{dH}_{2} \mathrm{O} \\ 1 \times \text { TAE buffer } & 40 \mathrm{mM} & \text { Tris-acetate } \mathrm{pH} 8.0 \\ & 1 \mathrm{mM} & \text { EDTA } \\ & \text { dissolved in } & \mathrm{dH}_{2} \mathrm{O}\end{array}$

\subsubsection{Cell culture methods}

\subsubsection{Culture of cells}

Cell lines were cultured in a $5 \% \mathrm{CO}_{2}$ humidified atmosphere at $37^{\circ} \mathrm{C}$. All cell culturing was done under sterile conditions in laminar flow cabinets and with sterile equipment. If not indicated otherwise, basal medium (DMEM + Glutamax with $10 \%$ (v/v) FCS) was used for cell culture. The sterility of medium additives was assured by manufacturers or achieved by autoclaving or sterile filtration through a $0.2 \mu \mathrm{m}$ filter unit, if necessary. Before usage, FCS was heated to $56^{\circ} \mathrm{C}$ for $30 \mathrm{~min}$ for inactivation of complement factors. Centrifugation of eukaryotic cells was performed at $1200 \mathrm{rpm}$ and RT for $5 \mathrm{~min}$, unless otherwise stated.

\subsection{Stem cell culture}

Stem cells were cultured on a monolayer of inactivated mouse embryonic fibroblasts (MEFs) or on Geltrex coated cell culture plates (333 $\mathrm{gg} / \mathrm{T} 25$ flask), prepared after manufacturer's instructions. The 
cells were splitted upon $80 \%$ confluence by washing once with PBS and subsequent incubation with stem cell trypsin for several minutes at $37{ }^{\circ} \mathrm{C}$ until dissociation of stem cell colonies occurred. A single cell suspension was prepared by diluting cells in 10 volumes of stem cell medium and gently pipetting up and down. About $1 / 10$ volume of this cells suspension was transferred into a new cell culture flask prepared with MEFs or Geltrex for further culture. Geltrex-coated flasks had to be incubated at $37^{\circ} \mathrm{C}$ for at least 30 min before usage, MEF coated flasks for 1 day in advance of stem cell passaging. Centrifugation of stem cells was always performed at $1000 \mathrm{rpm}$ for $5 \mathrm{~min}$. Stem cells were tested for fungal, bacterial and mycoplasma infection via PCR prior to experiments.

$\begin{array}{lll}\text { Stem cell medium } & \text { DMEM + Glutamax } \\ & +10 \%(\mathrm{v} / \mathrm{v}) \text { FCS } & \\ & +1 \%(\mathrm{v} / \mathrm{v}) 100 \times \text { NEAA } \\ & +50 \mu \mathrm{M} 2-\text {-mercaptoethanol } \\ & +1000 \mathrm{U} / \mathrm{ml} \text { LIF } & \\ & & \\ \text { Stem cell trypsin } & 0.1 \%(\mathrm{w} / \mathrm{v}) & \text { Glucose } \\ & 0.3 \%(\mathrm{w} / \mathrm{v}) & \text { Tris-HCl } \\ & 0.25 \%(\mathrm{w} / \mathrm{v}) & \text { Trypsin } \\ & 0.02 \%(\mathrm{w} / \mathrm{v}) & \text { EDTA } \\ & \text { dissolved in } & \text { PBS }\end{array}$

\subsection{Preparation of MEFs}

For generation of MEFs, an uterus of a pregnant mouse was dissected on day 13.5 and transferred into a cell culture dish with pre-warmed PBS. The embryos were removed and heads, extremities, tail and organs were discarded. Remaining parts were minced using forceps and incubated in an Erlenmeyer flask with sterile ballotinis and $5 \mathrm{ml}$ MEF trypsin (0.25\% (w/v) Trypsin; $0.02 \%$ (w/v) EDTA; dissolved in PBS) on a magnetic stirrer for $20 \mathrm{~min}$ at $37^{\circ} \mathrm{C}$. After washing with $10 \mathrm{ml}$ medium and centrifugation for $5 \mathrm{~min}$ at $1000 \mathrm{rpm}$, the pellet was resuspended in $25 \mathrm{ml}$ MEF medium and plated on a $15 \mathrm{~cm}$ cell culture dish. Following 3 days of culture, cells were frozen $\left(-150^{\circ} \mathrm{C}\right)$ or directly used for expansion and inactivation. For this, MEFs were expanded up to passage 4 in $15 \mathrm{~cm}$ cell culture dishes and upon confluence were inactivated with Mitomycin C (stock solution: $1 \mathrm{mg} / \mathrm{ml} \mathrm{PBS}$ ). After $2.5 \mathrm{hrs}$ incubation with $10 \mathrm{ml}$ inactivation medium (1\% (v/v) Mitomycin C stock solution in MEF medium), MEFs were washed twice with PBS and harvested by trypsinization. The cells were frozen in aliquots à $2 \times 10^{6}$ cells or directly plated for later usage on cell culture flasks treated with $0.1 \%(\mathrm{w} / \mathrm{v})$ gelatine for $15 \mathrm{~min}$ at RT. 


\subsection{Tumor cell culture}

Suspension tumor cells were cultured in MEF medium and maintained in the exponential growth phase by regular cell passaging twice a week. For this, approximately $1 / 10$ volume of the cell suspension was transferred into a new cell culture dish for further cell culture. Remaining cells were used for analysis or were discarded.

\subsection{Effector cell culture}

\subsection{MACS separation}

The magnetic-activated cell sorting (MACS) method allows the separation of various cell populations depending on their surface antigens by magnetic particles (magnetic MicroBeads) and a magnetic field. For isolation of NK cells, murine spleens were homogenized by a TenBrook homogenizer and isolated by MACS technology using a mouse NK cell isolation kit. TCR-transgenic T cells were isolated from homogenized lymph nodes using the mouse $\mathrm{CD} 4^{+} \mathrm{T}$ cell isolation kit or the $\mathrm{CD} 8 \mathrm{a}^{+} \mathrm{T}$ cell isolation kit, respectively. Cell isolation was accomplished by negative selection according to manufacturer's instructions. The cell solutions with the biotin-antibody cocktail and MicroBeads were transferred on MACS LS columns placed in a strong magnetic field of a MACS separator, catching magnetically labeled non-target cells on the column, whereas unlabeled NK or T cells pass through and were collected. Purity of obtained cells was determined by flow cytometry.

\subsection{NK cell culture}

NK cells were isolated by MACS separation (Ch. 3.2.2.1.3.1) and cultured in stimulation medium for 4 days in 6 well plates. The cell density ranged from $10^{6}$ to $10^{7}$ cells $/ \mathrm{ml}$.

$\begin{array}{ll}\text { NK cell stimulation medium } & \text { DMEM + Glutamax } \\ & +10 \%(\mathrm{v} / \mathrm{v}) \text { FCS } \\ & +50 \mu \mathrm{M} 2 \text {-mercaptoethanol } \\ & +10 \mathrm{ng} / \mathrm{ml} \mathrm{mlL}-2 \\ & +20 \% \text { Con A supernatant }\end{array}$




\subsection{Splenocyte cell culture}

For restimulation of splenocytes, spleens were extracted from animals and homogenized using a Tenbrook homogenizer. Cells were centrifuged and pellets were treated with $2 \mathrm{ml}$ erythrocyte lysis buffer for $3 \mathrm{~min}$. After an additional washing step, cell pellets were resuspended in DMEM supplemented with stimulation reagents and plated in 96 well plates for 4 days at $37^{\circ} \mathrm{C}$.

$\begin{array}{ll}\text { Restimulation medium for splenocytes } & \text { DMEM + Glutamax } \\ & +10 \%(\mathrm{v} / \mathrm{v}) \text { FCS } \\ & +50 \mu \mathrm{M} 2-\text { mercaptoethanol } \\ & +10 \mathrm{ng} / \mathrm{ml} \mathrm{mIL-2} \\ & +1 \mu \mathrm{M} \text { OVA } \\ & +20 \% \text { Con A supernatant }\end{array}$

To monitor the proliferation of splenocytes during the culturing, cells were previously stained with CFSE (Ch. 3.2.2.4). Unstained cells as well as portions of unstimulated cells were always cultured as reference cells, e.g. for flow cytometry. Cells were cultured in restimulation medium supplemented with OVA protein to final concentrations of $250 \mu \mathrm{M}$ for stimulation of CD4 ${ }^{+} \mathrm{T}$ cell from OT-II mice and $1 \mu \mathrm{M}$ for $\mathrm{CD}^{+} \mathrm{T}$ cells from OT-I mice.

$\begin{array}{lll}\text { Erythrocyte lysis buffer } & 155 \mathrm{mM} & \mathrm{NH}_{4} \mathrm{Cl} \\ & 10 \mathrm{mM} & \mathrm{KHCO}_{3} \\ & 0.1 \mathrm{mM} & \mathrm{EDTA}^{-1} \\ & \text { dissolved in } & \mathrm{dH}_{2} \mathrm{O}, \mathrm{pH} 7.2\end{array}$

\subsubsection{Freezing and thawing cells}

Cells were harvested from cell culture dishes depending on their specific growth conditions. After centrifugation, the cell pellets were resuspended in an adequate volume of cell-specific medium to reach a cell density around $1 \times 10^{6}$ cells $/ \mathrm{ml}$, of which $500 \mu$ l were transferred into a cryo conservation tube. After addition of an equal volume of $2 \times$ freezing medium (60 \% (v/v) cell-specific culture medium; $20 \%$ (v/v) DMSO; 20 \% (v/v) FCS), cryo tubes were slowly frozen in a closed styrofoam box at $-80^{\circ} \mathrm{C}$ for $24 \mathrm{hrs}$. For long-term storage tubes were transferred to $-150^{\circ} \mathrm{C}$. For thawing, cryo tubes with frozen cells were incubated briefly in a water bath at $37{ }^{\circ} \mathrm{C}$, diluted in washing medium and centrifuged for $5 \mathrm{~min}$. Subsequently, cells were resuspended in their specific growth medium and plated in an appropriate cell culture dish. 


\subsubsection{Counting of cells}

For cell counting, a Neubauer counting chamber and a Zeiss inverted microscope were used. After thorough resuspension of cells in an appropriate volume, either the suspension was directly used for counting or cells were mixed with $1 \%$ acetic acid to eliminate remaining erythrocytes in the solution (used for splenocyte cell suspensions). Alternatively, cells could be mixed with trypan blue counting solution (0.4 \% (w/v) trypan blue in PBS, pH 7.2), which selectively colors dead cells blue. The latter counting method is called trypan blue exclusion method, since living cells are excluded from staining. Counting was performed according to manufacturer's instructions of the Neubauer chamber by loading $10 \mu$ l of any cell suspension on the loading groove $(0.1 \mathrm{~mm}$ depth) between the chamber and the cover slip.

\subsubsection{CFSE staining of cells}

CFSE-stainings were performed to either monitor the proliferation capacity of in vitro restimulated splenocytes or to track stained cells after adoptive transfer into mice. Isolated splenocytes or TCRtransgenic T cells were incubated with CFSE according to manufacturer's instructions for $10 \mathrm{~min}$ in a water bath at $37^{\circ} \mathrm{C}$. Subsequently, cells were washed three times with basal medium followed by centrifugation at $1.200 \mathrm{rpm}$ for $5 \mathrm{~min}$.

\subsubsection{Immunological methods}

\subsubsection{Flow cytometry}

Flow cytometry is a laser-based, biophysical technology which allows rapid cell counting and detection of different characteristics of a single cell suspension. Endogenous fluorescence signals such as GFP or fluorescent dye-coupled antibodies, as well as cell size and granularity can be detected. Flow cytometry was performed with a FACSCalibur flow cytometer and CellQuest data acquisition software. Analysis was performed using FlowJo software. For measuring, single cells were collected from suspension cell culture or by trypsinization for adherent cells and washed with PBS. Approximately $1 \times 10^{6}$ cells per FACS tube were incubated with fluorescence-conjugated antibodies for $30 \mathrm{~min}$ at $4{ }^{\circ} \mathrm{C}$ in $100 \mu \mathrm{l}$ PBS to stain for different cell surface molecules. If unconjugated antibodies were used, an additional incubation with a fluorescence-conjugated secondary antibody was performed. Antibodies were used in dilutions according to manufacturer's instructions. After washing with PBS, cells were resuspended in an appropriate volume of PBS for measurement. Isotype controls were included for 
each experiment and fluorescent compensation of different fluorescent dye-conjugated antibodies was done with single-stained cell populations and unstained cells, respectively. In some cases, propidium iodide (PI) was added to the sample (10 $\mu \mathrm{M}$ final) in order to assess the viability of the cells.

\subsection{Intracellular cytokine measurements}

For intracellular cytokine measurements (ICM) via flow cytometry, splenocytes were stained with CD3-FITC and CD4-TC antibodies followed by a washing step with PBS. The cell pellets were then mixed with $2 \mathrm{ml}$ of BD Fix/Perm solution and incubated for $20 \mathrm{~min}$ at $4{ }^{\circ} \mathrm{C}$. After two washing steps with each $2 \mathrm{ml}$ of BD Perm/Wash solution, cells were stained with PE-coupled antibodies for intracellular cytokines for $30 \mathrm{~min}$ at $4{ }^{\circ} \mathrm{C}$. Subsequently, cells were washed once with BD Perm/Wash, followed by washing with PBS before analysis via the flow cytometer.

\subsubsection{2 ${ }^{51}$ Chromium release assay}

The ${ }^{51}$ Chromium $\left({ }^{51} \mathrm{Cr}\right)$ release assay is an in vitro method for quantification of cellular cytotoxicity, assessing the susceptibility of different target cells to cytotoxic immune effector cells. If killed,

${ }^{51} \mathrm{Cr}$-labeled target cells release the radioactive radionuclide, which can be measured in a liquid scintillation counter (LSC). For labeling of the targets, single cell suspensions of $1 \times 10^{6}$ or $5 \times 10^{5}$ cells were incubated with $200 \mu \mathrm{l} \mathrm{DMEM}$ containing $100 \mu \mathrm{lFCS}$ and $50 \mu \mathrm{Ci} \mathrm{Na}{ }_{2}^{51} \mathrm{CrO}_{4}$ for $1 \mathrm{~h}$ at $37^{\circ} \mathrm{C}$. Effector cells, i.e. NK cells or splenocytes, were plated in triplicates in serial dilutions on round-bottomed 96 well microtiter plates to achieve different effector to target (E:T) ratios. Labeled target cells were washed three times with PBS and added in numbers of $1 \times 10^{4}$ or $5 \times 10^{3}$ cells, respectively in DMEM $+10 \%$ FCS into the plates with a final volume of $200 \mu$ l per well. Spontaneous release was determined by incubation of target cells in the absence of effector cells. The microtiter plates were centrifuged for $5 \mathrm{~min}$ at $600 \mathrm{rpm}$ and incubated at $37^{\circ} \mathrm{C}$ for $4 \mathrm{hrs}$. After incubation, the plates were centrifuged again and $50 \mu$ of supernatant was transferred into Wallac plates. To break up the remaining cells and set all ${ }^{51} \mathrm{Cr}$ isotopes free, $10 \mu \mathrm{l} 10 \%$ Triton-X was added, mixed thoroughly and $50 \mu \mathrm{l}$ of this sediment part was transferred into separate Wallac plates. All probes were mixed with $200 \mu \mathrm{l}$ Scintillator and sealed before measurement in the MicroBeta ${ }^{2}$ Plate Counter. E:T ratios were adjusted depending on the percentage of the effector population measured via flow cytometric analysis. The percentage of specific lysis was calculated by subtracting the spontaneous ${ }^{51} \mathrm{Cr}$ release $\left({ }^{51} \mathrm{Cr}\right.$ release in absence of effector cells) shown in particular in the following formula: 


$$
[\%] \text { lysis }=\frac{4 \times \mathrm{cpm} \text { supernatant } \times 100}{3 \times \mathrm{cpm} \text { sediment }+1 \times \mathrm{cpm} \text { supernatant }}
$$

\subsubsection{ELISA}

Enzyme-linked immunosorbent assay (ELISA) is an analytic biochemical assay which can be used for detecting and quantifying substances like peptides or antibodies. With this method, the presence of OVA-specific antibodies in the sera of mice that previously received stem cell injections was analyzed in this study. ELISAs were performed in 96-well Nunc MaxiSorртм microtiter plates and analyzed with a BioTek PowerWave 340 microplate spectrophotometer. At first, 96-well plates were coated overnight with $1 \mu \mathrm{g}$ OVA per well diluted in $50 \mu \mathrm{l}$ carbonate buffer at $4{ }^{\circ} \mathrm{C}$, followed by an incubation with $150 \mu \mathrm{l}$ of $1 \%(\mathrm{w} / \mathrm{v})$ gelatine dissolved in PBS for $1 \mathrm{hr}$ at $37^{\circ} \mathrm{C}$ to ensure the blocking of unspecific binding sites. After the dilution of sera with PBS/Tween, $50 \mu \mathrm{l}$ of these were incubated in the prepared 96-well plates for $2 \mathrm{hrs}$ at $37^{\circ} \mathrm{C}$. The plates were then washed three times with $\mathrm{H}_{2} \mathrm{O}$ and $50 \mu \mathrm{l}$ peroxidase conjugated goat anti-mouse IgG (1:5000 in PBS/Tween) antibody solution per well was added to the plates. In order to differentiate between Ig isotypes in the sera, isotype-specific secondary antibodies were additionally used, which were detected with a peroxidase-conjugated goat anti-rat antibody. The plates were again incubated at $37^{\circ} \mathrm{C}$ for $1 \mathrm{hr}$ and washed 5 times with $\mathrm{H}_{2} \mathrm{O}$ before $100 \mu \mathrm{l}$ substrate solution per well was added. After $5 \mathrm{~min}$, the maximum absorbance at $405 \mathrm{~nm}$ was detected in the ELISA reader.

\begin{tabular}{|c|c|c|}
\hline Carbonate buffer & $\begin{array}{l}85 \mathrm{ml} \\
40 \mathrm{ml} \\
375 \mathrm{ml}\end{array}$ & $\begin{array}{l}\mathrm{Na}_{2} \mathrm{CO}_{3}(0.2 \mathrm{M}) \\
\mathrm{NaHCO}_{3}(0.2 \mathrm{M}) \\
\mathrm{dH}_{2} \mathrm{O}\end{array}$ \\
\hline PBS/Tween & & PBS \\
\hline & $0.05 \%(v / v)$ & Tween \\
\hline Substrate buffer & $\begin{array}{l}0.1 \mathrm{M} \\
0.05 \mathrm{M}\end{array}$ & $\begin{array}{l}\mathrm{C}_{2} \mathrm{H}_{3} \mathrm{NaO}_{2} \\
\mathrm{NaH}_{2} \mathrm{PO}_{4} \mathrm{pH} 4.0\end{array}$ \\
\hline Substrate solution & $\begin{array}{l}10 \mathrm{ml} \\
500 \mu \mathrm{l} \\
10 \mu \mathrm{l}\end{array}$ & $\begin{array}{l}\text { substrate buffer } \\
\text { ABTS }(40 \mathrm{mM}) \\
\mathrm{H}_{2} \mathrm{O}_{2}(30 \%)\end{array}$ \\
\hline
\end{tabular}




\subsubsection{Histology}

\subsubsection{Tissue cross-sections}

Previously fixed teratoma and heart tissue were dehydrated in a series of ethanol solutions of increasing concentrations until $100 \%$ (50\%, $70 \%, 80 \%, 2 \times 96 \%, 2 \times 100 \%$ for 75 min each), since paraffin is immiscible with water. Then, the ethanol was replaced with xylol $(2 \times 75 \mathrm{~min})$, which was exchanged by liquid paraffin wax $\left(56-58^{\circ} \mathrm{C}\right)$ and the samples were subsequently embedded into paraffin blocks. Thin slices of $2.5 \mu$ l (teratoma) to $5 \mu \mathrm{m}$ (heart) from pre-chilled blocks were generated using a microtome and transferred to glass slides. These were dried overnight at $37^{\circ} \mathrm{C}$ and stored at $\mathrm{RT}$ for subsequent analysis.

\subsubsection{Hematoxylin and eosin staining}

For histopathological examination, paraffin sections were stained with Hematoxylin and eosin (H\&E) to get a broad overview of different cell types within the sample by differentiating between cytoplasm and cell nuclei. Hematoxylin (hematein) is considered as a basic dye, which stain acidic or basophilic structures a purplish blue (e.g. DNA, RNA), whereas eosin, as an acidic dye, stains basic or acidophilic structures red or pink (e.g. cytoplasmic proteins). Dried tissue sections were deparaffinized with xylol ( $2 \times 7 \mathrm{~min})$, rehydrated in a descending alcohol series (100\%, $96 \%, 75 \%$ and $60 \%$ ethanol for $5 \mathrm{~min}$ each) and washed with $\mathrm{dH}_{2} \mathrm{O}$ for 5 min. The samples were then incubated in Mayer's hemalum solution for 5 min, rinsed with $\mathrm{H}_{2} \mathrm{O}$ for 10 min and incubated in eosin solution (1 \% Eosin, $200 \mu$ acetic acid, $\mathrm{dH}_{2} \mathrm{O}$ up to $100 \mathrm{ml}$ ) for additional $5 \mathrm{~min}$. Subsequently, samples were briefly washed with $\mathrm{dH}_{2} \mathrm{O}$ followed by incubation in an ascending series of alcohol (60\%, $75 \%, 96 \%, 100 \%)$ and isopropanol (5 min). Sections were transferred to xylol $\left(2 \times 5 \mathrm{~min}\right.$ ) prior to embedding using Roti $^{\circledR}{ }^{-}$Histokitt. For staining of specific proteins, additional immunohistochemistry was performed.

\subsubsection{Sirius Red staining}

For collagen stainings of myocardia, tissue sections were stained for $1 \mathrm{hr}$ at RT in Sirius Red solution, i.e. $0.1 \%$ Direct Red 80 in saturated aqueous picric acid adjusted to $\mathrm{pH} 2.0$ with sodium hydroxide. After tipping in $0.5 \%$ acetic acid solution for four times, slides were incubated in ethanol ( $1 \times 5 \mathrm{~min})$, isopropanol (1 × $5 \mathrm{~min}$ ) and xylol $(2 \times 10 \mathrm{~min})$. Subsequently, slides were embedded using Roti ${ }^{\circledR}$ Histokitt, scanned with a dotSlide and analyzed with the cellSens dimensions software. 


\subsubsection{Immunohistochemistry}

For Immunohistochemistry (IHC), paraffin sections were stained with specific antibodies to detect proteins of interest. All antibodies were diluted according to the manufacturer's instructions and were incubated in a humidified atmosphere on the slides to prevent dehydration of the tissue samples. First, tissue sections were processed as for H\&E staining (Ch. 3.2.4.2) but after rehydration, sections were boiled in citrate buffer $(3 \times 5 \mathrm{~min} ; 10 \mathrm{mM}, \mathrm{pH}$ 6.0) for antigen retrieval. After cooling down to RT, samples were incubated with IHC blocking solution (4 \% BSA in PBS) for $1 \mathrm{hr}$ at RT. The primary antibody was diluted in PBS with $1 \%$ BSA and incubated overnight at $4{ }^{\circ} \mathrm{C}$. To block endogenous peroxidases, tissue sections were incubated in $3 \% \mathrm{H}_{2} \mathrm{O}_{2}(30 \%)$ in PBS at $\mathrm{RT}$ for 10 min and slides were subsequently washed with PBS ( $3 \times 5 \mathrm{~min}$ ) before incubation with the secondary biotin-conjugated antibody for $1 \mathrm{hr}$ at RT. After additional washings with PBS ( $3 \times 5 \mathrm{~min}$ ), samples were incubated with HRP-streptavidin for $1 \mathrm{hr}$ at RT and stained with a DAB substrate solution $\left(0.05 \% \mathrm{DAB}, 0.015 \% \mathrm{H}_{2} \mathrm{O}_{2}\right.$, dissolved in PBS). For a hematoxylin counterstaining, the samples were incubated in hematoxylin (3 min), rinsed with $\mathrm{H}_{2} \mathrm{O}(5 \mathrm{~min})$ and dehydrated before the tissue slides were mounted with Roti $^{{ }_{-}}$ Histokitt for imaging. Stained slides were scanned with a dotSlide and further analyzed using the cellSens dimensions software.

\subsubsection{Animal experiments}

\subsubsection{Animal monitoring}

Animals were monitored for the complete duration of experiments. Every second day, weight of operated mice was controlled. The loss of more than $20 \%$ weight in $48 \mathrm{hrs}$ or an altered outer appearance let to preterm ending of the experiment. Mice with inoculated stem cells were examined for tumor growth every third day and the mice were sacrificed when a tumor volume of $1 \mathrm{~cm}^{3}$ was reached or any signs of pain or discomfort were recorded.

\subsubsection{Teratoma assays}

To analyze the tumorigenicity and immunogenicity of stem cell lines, cells were inoculated in syngeneic immunocompetent mice. For this, stem cells were harvested, counted and washed with PBS. Each mouse received amounts of $1.5 \times 10^{6}$ to $3 \times 10^{6}$ cells, resuspended in $100 \mu \mathrm{l}$ PBS via subcutaneously (s.c.) injections into the flank. Tumor growth was monitored for up to 90 days and regularly controlled by palpation. 


\subsubsection{Adoptive transfer of TCR-transgenic T cells}

TCR-transgenic T cells were isolated from OT-I and OT-II mice via MACS technology. After CFSEstaining, $1.5 \times 10^{6}$ to $12 \times 10^{6} \mathrm{~T}$ cells, depending on the experimental setup, were dissolved in approximately $100 \mu \mathrm{l}$ PBS. Mice were fixed in a special self-made $50 \mathrm{ml}$ falcon with breathing holes and notch for the tail. Before intravenous (i.v.) injection, mice tails were stuck into warm water which lead to dilation of blood vessels and as a consequence to a better visualization of the tail veins. Cells were injected into a lateral vein of the mouse tail.

\subsubsection{Transverse aortic constriction and echocardiography}

TAC surgery was performed by CRC 1002 service project using a minimally invasive operation procedure previously described in Toischer et al. (2010). For this, 8 to 12 weeks old mice were anesthetized via intraperitoneal injections of medetomidin $(0.5 \mathrm{mg} / \mathrm{kg})$, midazolam $(5 \mathrm{mg} / \mathrm{kg})$, and fentanyl $(0.05 \mathrm{mg} / \mathrm{kg})$. Pressure overload was induced by transversal aortic constriction using a 26 gauge needle. Hereby, the diameter of the aorta was typically reduced by approximately $50 \%$ to create a 50 to $60 \mathrm{mmHg}$ systolic pressure gradient between the left ventricle (LV) and aortic pressure. However the gradient might vary depending on hemodynamic conditions and the severity of the aortic ligation. In sham operations, the transversal aorta was displayed but not restricted. Anesthesia was reversed by s.c. injections of atipamezol $(2.5 \mathrm{mg} / \mathrm{kg})$ and flumazenil $(0.5 \mathrm{mg} / \mathrm{kg})$. Mice were kept on heating plates until full recovery from anesthesia. For analgesia, drinking water was supplemented with $1.33 \mathrm{mg} / \mathrm{ml}$ metamizole two days before surgery, which was refreshed every third day until one week after operation. Three days after surgery, the pressure gradient over the aortic ligature (aortic stenosis) was measured by transthoracic echocardiography using a Vevo2100 system with a $30 \mathrm{MHz}$ center frequency transducer. Therefore, animals were anesthetized with $3 \%$ isoflurane, and respiration, temperature and ECG-controlled anesthesia was maintained with $1.5 \%$ isoflurane, as described previously (Toischer et al., 2010). At additional points of time after operations (1 week, 4 weeks, 8 weeks), echocardiography was performed to determine the maximal left ventricular length (L), thicknesses of the septum, the posterior myocardial wall, the inner diameter of the left ventricle (LVEDD) and the area of the left ventricular cavity (area). Fractional area shortening (FAS) and ejection fraction (EF) were calculated via the area length method. 


\subsubsection{Sacrification and tissue preparation}

Animals were generally sacrificed in a $\mathrm{CO}_{2}$ atmosphere, followed by cervical dislocation. Therefore, an animal was placed into a suitable chamber (e.g. IVC) which was initially filled with atmospheric air, followed by gradually filling with $\mathrm{CO}_{2}$ for some minutes until unconsciousness and death of the animal. Anesthetized animals were disinfected with $70 \%$ ethanol before dissection.

\subsection{Donor animals}

For the analysis and isolation of effector cells, murine lymph nodes or/and spleens of rat and mice were extracted with a sterile dissection set and cooled in DMEM falcons on ice until homogenization. Lymph nodes, used for isolation of OT-I and OT-II TCR-transgenic T cells, were collected from lateral flanks, cervical neck and from the large intestine and lower abdomen.

\subsection{Preparation of Concanavalin A supernatant from rat spleens}

Concanavalin (Con) A supernatant is used as supplement in stimulation-media for splenocytes. To generate the supernatant, several rat spleens were dissected and homogenized by a Tenbrook homogenizer, followed by an $4 \mathrm{hrs}$ incubation in specific Con A stimulation medium (DMEM, 5 \% FCS, $200 \mu \mathrm{l}$ Con A) at $37{ }^{\circ} \mathrm{C}$. Afterwards, splenocytes were collected, washed with DMEM and further cultured in DMEM supplemented with $10 \%$ FCS for $24 \mathrm{hrs}$ in a cell incubator. The stimulated lymphocytes secret various cytokines during this incubation time, generating a cytokine conditioned supernatant which is collected after centrifugation (10 min; $1100 \mathrm{xg}$ ), aliquoted and stored at $-20^{\circ} \mathrm{C}$.

\subsection{TCR-transgenic T cells from OT-I or OT-II mice}

OT-I and OT-II mice are T-cell receptor (TCR)-transgenic animals, whose T cells were designed to recognize specific chicken ovalbumin (OVA) residues. The $\mathrm{CD} 8^{+} \mathrm{T}$ cells of OT-I mice recognize the OVA residues $257-264$ in the context of $\mathrm{H} 2 \mathrm{~Kb}$ ( $\mathrm{H} 2$ class I) and the $\mathrm{CD}^{+}{ }^{+} \mathrm{T}$ cells of OT-II mice the OVA residues 323-339 in the context of I-A (H2 class II). Therefore, OT-I T cells were used to study the response of cytotoxic T cells to antigen, whereas the OT-II mice were generated to analyze T cell help for B cell responses towards OVA as model antigen. To isolate TCR-transgenic T cells, lymph nodes from OT-I or OT-II mice were homogenized with a Ten brook homogenizer and cells were passed through a cell strainer. Cells were counted and an appropriate amount was used for subsequent MACS separation. 
The isolated cells were counted and stained with CFSE and subsequently used for adoptive transfer. Cell quality was assured via flow cytometry.

\subsection{Preparation of teratomas}

Subcutaneous teratomas were prepared from anesthetized animals, measured, weighted and cut into three parts. The first piece was immediately transferred to liquid nitrogen and stored at $-80{ }^{\circ} \mathrm{C}$ for later gene expression analyses. Another part of the tumor sample was fixed in phosphate-buffered $4 \%$ formalin overnight at $4{ }^{\circ} \mathrm{C}$, followed by paraffin embedding for histological analysis. The other piece was stored in DMEM on ice for subsequent flow cytometric analysis. The abdomen and thoracic cavity were examined systematically for the presence of metastases, which, if present, were also treated as s.c. teratoma samples. After autopsy, tumor size was measured by calipers and its volume was calculated with the following formula:

$$
V=\frac{\pi \times a \times b \times c}{2}
$$

$a, b, c=$ orthogonal diameters

\subsection{Teratoma digestion for flow cytometric analysis}

For flow cytometric analysis of teratomas, tumors were digested with Liberase, which is composed of highly purified collagenase I and collagenase II. First, tumors were cut into small pieces and transferred to round bottomed falcons in which small magnetic stirring bars were placed. A small volume of digestion medium (DMEM w/o FCS supplemented with $0.12 \mathrm{mg} / \mathrm{ml}$ Liberase) was added and the tubes were set into a $37{ }^{\circ} \mathrm{C}$ water bath onto a magnetic stirrer and incubated for $10 \mathrm{~min}$. After the first incubation, the supernatant was transferred into a fresh tube with ice-cold medium with FCS and another volume of digestion medium was added to the remaining tumor parts. After additional $10 \mathrm{~min}$ and merging of both supernatants, tubes were centrifuged at $1200 \mathrm{rpm}$ for $10 \mathrm{~min}$, followed by erythrocyte lysis for $3 \mathrm{~min}$ and an additional washing step with DMEM medium at $1200 \mathrm{rpm}$ for $10 \mathrm{~min}$. The cell suspension was then passed through a $40 \mu \mathrm{m}$ nylon mesh and centrifuged again for $10 \mathrm{~min}$ at $1200 \mathrm{rpm}$. The resulting pellet was washed once with PBS before addition of antibodies for flow cytometry. 


\subsection{Blood and sera preparation}

After animals were sacrificed, blood was directly taken by cardiopuncture using a $1 \mathrm{ml}$ syringe and transferred to $1.5 \mathrm{ml}$ Eppendorf tubes for sera generation or in EDTA-containing plastic tubes if analysis using flow cytometry was required. Eppendorf tubes were initially stored at RT, followed by storage at $4{ }^{\circ} \mathrm{C}$ for 2-3 hrs. After centrifugation for $10 \mathrm{~min}$ at $1200 \mathrm{rpm}$, the upper phase was transferred into a new tube, the procedure was once repeated and pure sera were stored for subsequent analysis at $-20^{\circ} \mathrm{C}$. For flow cytometry analysis, blood was directly transferred from EDTAcontaining tubes into FACS tubes and stained with antibodies. After incubation, erythrocytes were lysed with FACS ${ }^{\mathrm{m} M}$ Iysis solution according to manufacturer's instructions, followed by washing with PBS and subsequent measurement via flow cytometry.

\subsection{Preparation of TAC- and sham-operated animals}

After anesthesia and heart punctuation for blood isolation, hearts were excised, weighted and perfused with saline solution to eliminate residing blood in the tissue. The atria were cut off and the ventricles were weighted and dissected into three parts of which all were immediately transferred to liquid nitrogen. Samples were stored at $-150^{\circ} \mathrm{C}$ until usage for gene expression or histological analysis. Additionally, the length of tibia was measured for later calculations of the normalized heart weight (heart weight / tibia length). Spleens were collected in DMEM medium for later processing.

\subsubsection{Statistical analysis}

Statistical analysis was performed using WinStat software. For statistical evaluation of ${ }^{51} \mathrm{Cr}$ release assays, mainly the two-way-ANOVA was used to analyze the lysis of different target cell lines by cytotoxic effector cells. Otherwise, if data were not normally distributed, the non-parametric KruskalWallis $(\mathrm{H})$ test was used for comparison of multiple groups, or the Mann-Whitney U-test for comparison of two groups. In single cases, the chi-squared test or student's t-test was used. Applied tests are indicated in figure legends. Data are depicted as mean + standard deviation (SD) or standard error of the mean (SEM). A $p$ value of less than $0.05\left({ }^{*}\right)$, less than $0.01\left({ }^{* *}\right)$ or less than $0.001\left({ }^{* * *}\right)$ was considered statistically significant. 


\section{Results}

\subsection{Investigation of immune response contributing to the pathogenesis of load-induced heart failure}

Due to the high prevalence of heart failure (HF), it is important to understand the underlying mechanisms to prevent or treat this disease. One new concept of treatment could be the transplantation of stem cell-derived grafts. For this, it is important to examine the immunological milieu of the diseased heart, which would presumably influence the outcome of transplantations. Moreover, autoimmunity can contribute to the development of $\mathrm{HF}$ and new results suggest that even purely load-induced HF might be associated with the development of autoimmune reactions. Therefore, we used cMy-mOVA mice, which express ovalbumin (OVA) as model antigen in cardiomyocytes to examine whether HF induced by increased afterload elicits OVA-specific autoimmunity. For this, animals underwent transverse aortic constriction (TAC) surgery to increase the afterload of the heart. Previous experiments in our research group had revealed that cMy-mOVA mice show a small but statistically significant increase of OVA-specific CTL activity after TAC operation as tested in ${ }^{51}$ chromium $\left({ }^{51} \mathrm{Cr}\right)$ release assays but with considerable individual variations. The level of OVA-specific antibodies was not increased after TAC operation and generally the heart function was not altered compared to wildtype mice as determined by echocardiography (Röhrborn et al., unpublished data). These findings indicate that clinically relevant autoimmunity against OVA as a model of a cardiac autoantigen was not a frequent outcome of TAC operations in our experimental setting. Therefore, animals were now further challenged by adoptive transfer of OVA-specific T cells to increase the number of naïve autoreactive T cells and to examine the progression of HF in animals at higher risk to develop autoimmunity. 


\subsubsection{Adoptive transfer of TCR-transgenic T cells into cMy-mOVA mice}

OVA-specific TCR-transgenic T cells of OT-I $\left(C D 8^{+}\right)$and OT-II $\left(C D 4^{+}\right)$mice were isolated by MACS technology and stained with CFSE before being adoptively transferred intravenously $\left(10^{7}\right.$ cells in total /mouse) into cMy-mOVA mice. Experiments were performed in which both OT-I and OT-II T cells were transferred together because autoantigen-specific $\mathrm{CD} 4^{+}$and $\mathrm{CD} 8^{+} \mathrm{T}$ cells might need to cooperate to accelerate HF. In addition, transfers of only OT-I T cells were performed, because we had previously observed a significant activation of OVA-specific CTLs in cMy-mOVA mice after TAC. Transfers of OT-II T cells are on the way but currently not sufficient in numbers to be presented here. Animals underwent TAC or sham operation (day 3 post transfer) and functional parameters of the heart were regularly monitored via echocardiography. Usually at day 3 after the operations, the stenosis of the aorta was determined by echocardiography in millimeters of mercury $(\mathrm{mmHg})$. Generally, TAC-operated animals showed stenosis of $>50 \mathrm{mmHg}$, whereas sham-operated control animals showed values about 2-5 $\mathrm{mmHg}$ (data not shown).

To identify the transferred $\mathrm{CFSE}^{+} \mathrm{T}$ cells, blood was taken at day 6 post transfer. Flow cytometric analysis showed that CFSE ${ }^{+}$cells constituted only a minor proportion of PBMCs in the blood (Figure 7A). In single animals up to $1.2 \% \mathrm{CFSE}^{+}$T cells $\left(\mathrm{CD}^{+} \mathrm{CFSE}^{+}\right)$of total PBMCs were found in the blood at that time point. Notably, much less $\mathrm{CD}^{+} \mathrm{CFSE}^{+} \mathrm{T}$ cells were found than $\mathrm{CD} 8^{+} \mathrm{CFSE}^{+} \mathrm{T}$ cells. Generally, TAC-operated animals showed lower numbers of $\mathrm{CFSE}^{+}$cells in the blood than sham-operated animals. This might indicate recruitment to lymphoid organs or the heart, or loss of the CFSE signal due to proliferation. Animals were monitored up to 10 weeks after surgery, depending on the health condition of mice. At the end point they were sacrificed and tissue samples, including blood, spleen and heart were collected for analysis. Blood analysis of animals, which had received a mixture of OT-I and OT-II T cells showed a minimal amount of $\mathrm{CFSE}^{+}$cells in the lymphocyte population in flow cytometry (Figure 7B). The low numbers of $\mathrm{CFSE}^{+}$cells at this late time point could indicate loss of cells, recruitment to other organs or proliferation. 
Screening for CFSE-labeled T cells in blood samples after adoptive transfer

A

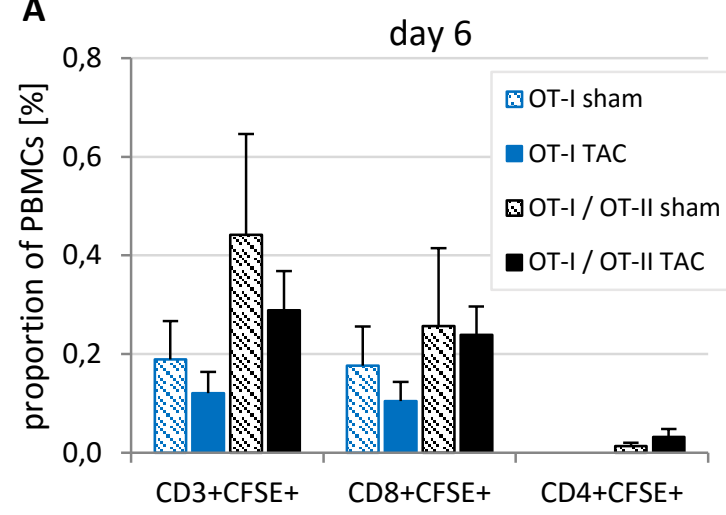

B

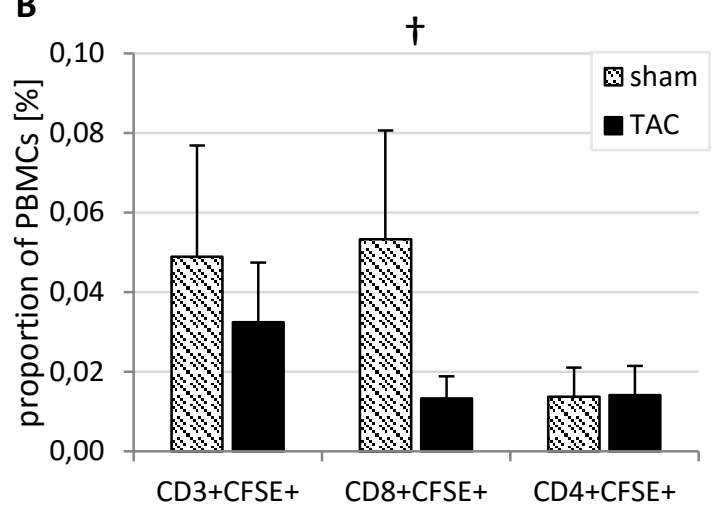

Figure 7 Flow cytometric analysis of transferred T cells in blood samples of $\mathrm{cMy}$-mOVA mice which received TCR-specific OT-I T cells ( $10^{7}$ cells /mouse) or a mixture of OT-I and OT-II T cells (5 x 10 $10^{6}$ each $\left./ \mathrm{mouse}\right)$. Shown is the proportion of CFSE-stained cells of total lymphocyte numbers + SEM. (A) Proportion of CFSE ${ }^{+}$ T cells of total PBMC numbers in blood samples taken 6 days post transfer. Animal numbers of CD3, CD8 and CD4 measurements respectively: OT-I sham: CFSE/CD3 $(n=9)$, CFSE/CD8 $(n=9)$, CFSE/CD4 $(n=0)$; OT-I TAC: CFSE/CD3 $(n=9)$, CFSE/CD8 $(n=9)$, CFSE/CD4 $(n=0)$; OT-I/OT-II sham: CFSE/CD3 $(n=7)$, CFSE/CD8 $(n=7)$, CFSE/CD4 $(n=3)$; OT-I /OT-II TAC: CFSE/CD3 $(n=8)$, CFSE/CD8 $(n=9)$, CFSE/CD4 $(n=5)$ (B) Proportion of CFSE ${ }^{+}$ T cells of total PBMC numbers after OT-I/OT-II transfer in blood samples taken after experimental endings (67 days). Animal numbers of performed CD3, CD8 and CD4 measurements respectively: sham: CFSE/CD3 $(n=9)$, CFSE/CD8 $(n=9)$, CFSE/CD4 $(n=8) ;$ TAC: CFSE/CD3 $(n=12)$, CFSE/CD8 $(n=12)$, CFSE/CD4 $(n=12)$.

Analysis of the spleen for CFSE-stained cells at the end of the experiments showed that up to $0.89 \%$ of splenocytes in a single animal (TAC, OT-I T cell transfer, $\mathrm{CD}^{+} \mathrm{CFSE}^{+}$) were positive for CFSE. In general, only very few $\mathrm{CFSE}^{+} \mathrm{T}$ cells were identified (Figure 8A). TCR-transgenic T cells of OT-I and OT-II mice can be identified by an anti-TCR V $\beta$ 5.1/5.2 mAb, which is specific for the V $\beta$ chain that was used for the transgenic TCR of OT-I and OT-II mice. Between $2.0 \%$ to $2.6 \%$ of splenocytes were positive for TCR V $\beta$ 5.1/5.2, indicating a normal range of $T$ cells with usage of these $V \beta$ chains. Thus, the transferred $\mathrm{CD} 8^{+} \mathrm{T}$ cells did not undergo a massive expansion at least not within the splenocyte population (Figure 8B). The OT-II mice were CD45.1 $1^{+}$, whereas the OT-I and cMy-mOVA mice expressed the CD45.2 allotype. This enabled a tracking of the transferred OT-II cells by a stable genetic marker. CD $45.1^{+}$cells originating from OT-II mice constituted on average $0.06 \%$ (sham) to $0.21 \%$ (TAC) of the splenocyte population of cMy-mOVA mice after transfer of OT-I and OT-II T cells. Thus, also the transferred $\mathrm{CD}^{+} \mathrm{T}$ cells did not undergo a massive expansion at least not within the splenocyte population. 

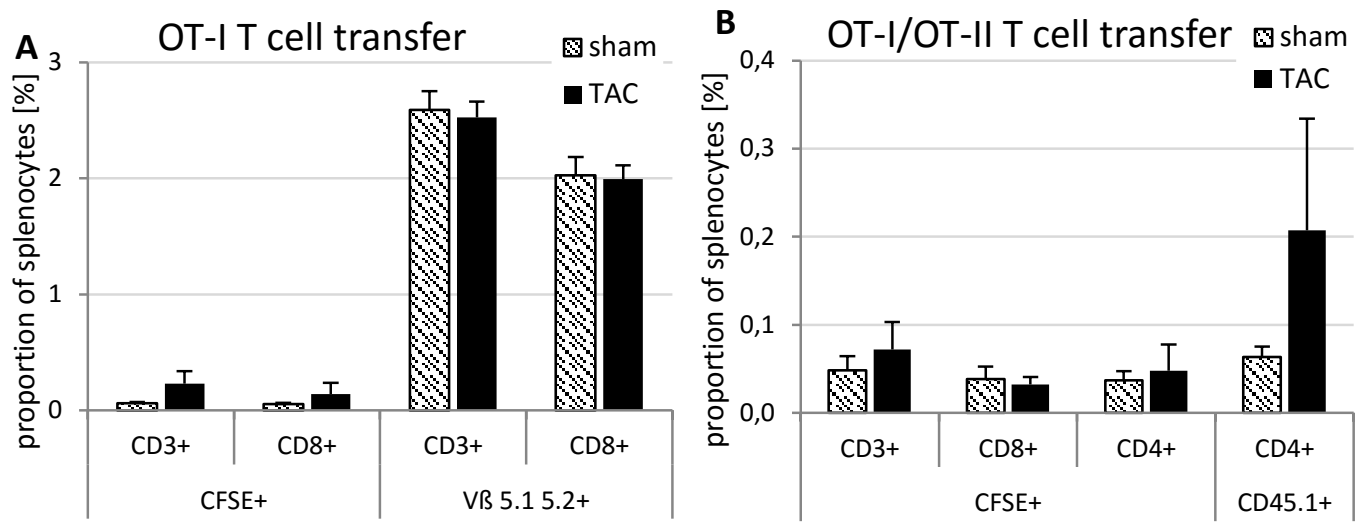

Figure 8 Flow cytometric analysis of transferred T cells in spleens of $\mathrm{cMy}$-mOVA mice which received OT-I T cells ( $10^{7} /$ mouse) or a mixture of OT-I and OT-II T cells $\left(5 \times 10^{6}\right.$ each /mouse). Shown is the percentage of positive cells of total splenocytes + SEM (A) Proportion of CFSE ${ }^{+}$and TCR V $\beta$ 5.1/5.2 $2^{+}$cells of total splenocytes of animals which received OT-I T cells at the end of experiments. CFSE/CD3 and CFSE/CD8: sham $(n=7)$, TAC $(n=8) ;$ TCR V $\beta$ 5.1/5.2 /CD3 and TCR V $\beta$ 5.1/5.2 /CD8: sham ( $n=7)$, TAC $(n=7)$ (B) Proportion of $\mathrm{CFSE}^{+}$and CD45.1 $1^{+}$cells of total splenocytes after experimental ending of animals which received OT-I and OT-II T cells. Animal numbers: sham $(n=6), \operatorname{TAC}(n=10)$.

\subsubsection{Immunohistochemical analysis of leukocyte infiltration into the myocardium}

Lymphocyte infiltration into of the myocardium was determined on paraffin-embedded cross sections of the heart by immunohistochemistry $(\mathrm{IHC})$. Example pictures in Figure 9 show an infiltration of $\mathrm{CD}^{+}$ and $\mathrm{CD}_{45 \mathrm{R}^{+}}$cells into the myocardium of cMy-mOVA mice, which received OVA-specific $\mathrm{T}$ cells by adoptive transfer. Additionally, the type of infiltrating T cells was determined by staining for $\mathrm{CD}^{+}$and $\mathrm{CD}^{+}$cells (not shown). Stained tissue slides were scanned and analyzed via cellSens dimension software, determining cell counts per scanned tissue section area. 


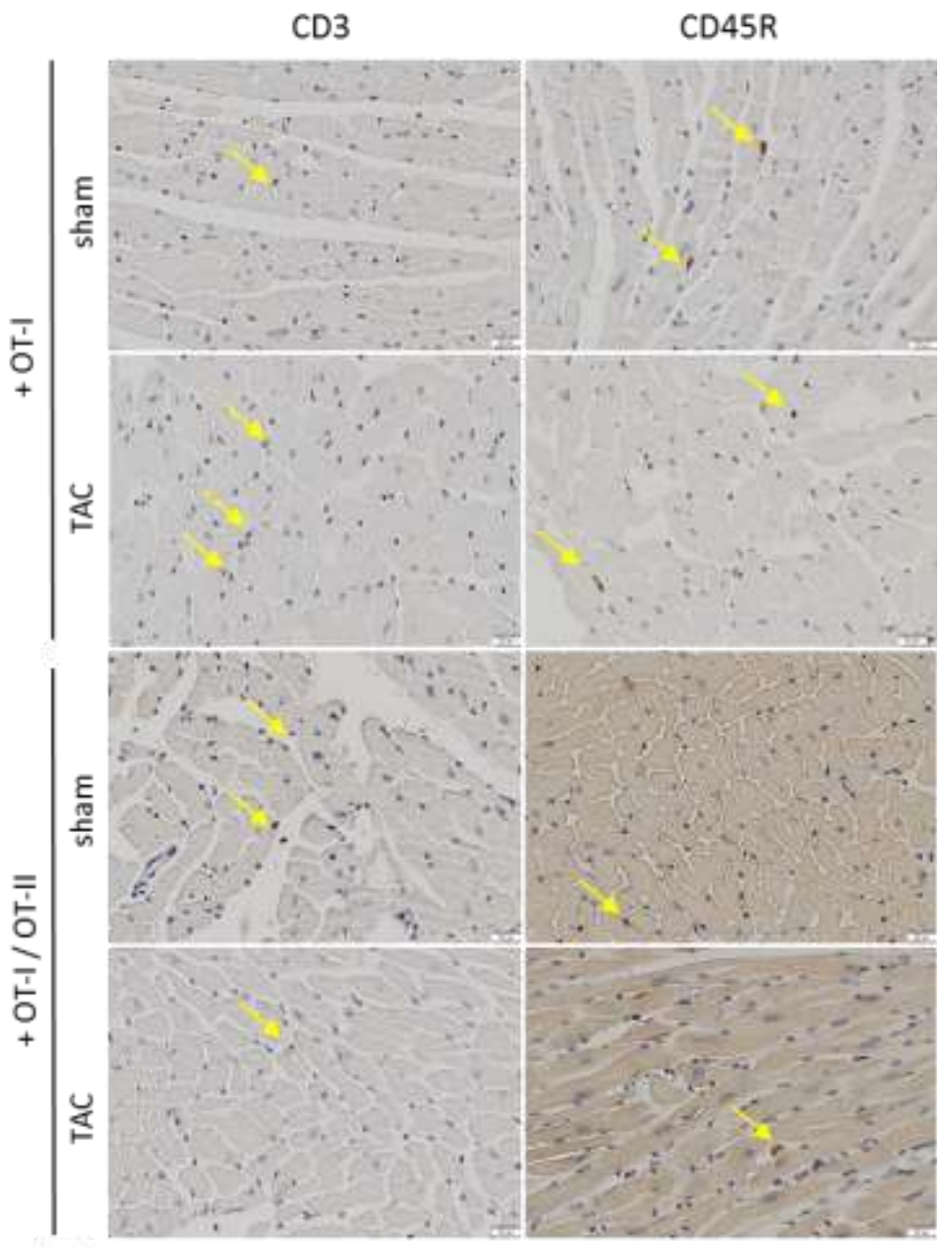

Figure 9 IHC analysis of lymphocyte infiltration into myocardia of cMymOVA mice, which received either OT-I T cells or OT-I and OT-II T cells. Shown are histological example tissue slides of TAC- and shamoperated animals stained for CD3 or CD45R. Arrows indicate positive stained cells. Scale bars in each picture (bottom right corner) represent $20 \mu \mathrm{m}$ each.

Due to variations in size of myocardial sections, cell counts per $\mathrm{mm}^{2}$ myocardium were computed, shown in Figure 10. The most abundant lymphocytes were $C D 8^{+}$(i.e. cytotoxic T cells) and CD45R ${ }^{+}$(i.e. B cells) (Figure $10 \mathrm{~A}$ ). $\mathrm{CD}^{+}$and $\mathrm{CD}^{+}$cells were only found in lower numbers in the myocardium of cMy-mOVA mice, ranging from $3.3 \times 10^{-3}$ to 0.01 cells per $\mathrm{mm}^{2}$ myocard. Generally, more lymphocytes were detected in TAC-operated animals than in sham-operated animals and the amount of infiltrating T cells in the myocardium was always increased in animals which received adoptive transfers of T cells when summarizing transfer experiments of OT-I and OT-I/OT-II T cells. The higher frequency of CD8 ${ }^{+}$ than $\mathrm{CD}^{+} \mathrm{T}$ cells could indicate differences in the quality of the mAbs or the presence of $\mathrm{CD} 8^{+} \mathrm{CD} 3^{-}$ cells, e.g. some NK cells or DCs. 
$\mathrm{IHC}$ analysis of lymphocyte infiltration into the myocardium
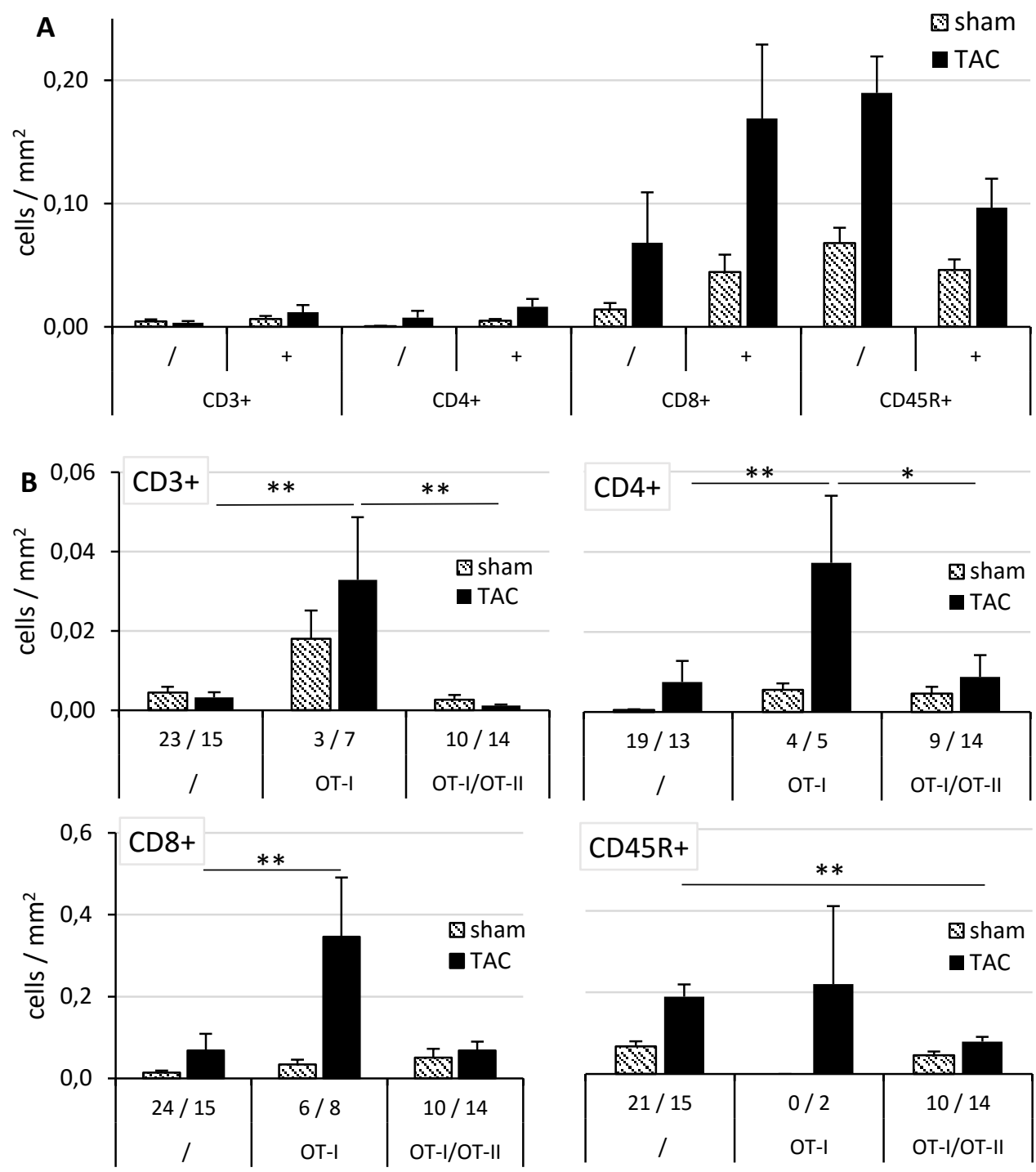

Figure 10 Immunohistochemical analysis of the myocardium of control cMy-mOVA mice and cMy-mOVA mice which received TCR-transgenic T cells.

Shown are the mean of $n$-experiments ( $n$ numbers depicted below the columns in (B)) + SEM. (A) Summary of IHC analysis of infiltrated lymphocytes in the myocardium of operated (TAC, sham) cMy-mOVA mice which received no T cells (/) and cMy-mOVA mice which received T cells (OT-I and OT-I/OT-II) (+). (B) Diagrams of lymphocyte numbers in the myocardium of different animal groups (control, OT-I transfer, OT-I/OT-II transfer) which underwent sham or TAC surgery. Significant differences between groups of TACoperated animals are depicted in the diagram: ${ }^{*}<0.05,{ }^{* *}<0.01$. Differences between TAC-operated cMymOVA and cMy-mOVA + OT-I T cells: $\mathrm{CD}^{+}\left(p=9.3 \times 10^{-3}\right), \mathrm{CD}^{+}\left(p=8.5 \times 10^{-3}\right), \mathrm{CD}^{+}\left(p=4.4 \times 10^{-3}\right), \mathrm{CD}^{+} 5 \mathrm{R}^{+}$ $(p=0.99)$, between TAC-operated cMy-mOVA and cMy-mOVA + OT-I/OT-II T cells: $\mathrm{CD3}^{+}(p=0.75), \mathrm{CD}^{+}(p$ $=0.29), \mathrm{CD}^{+}(p=0.09), \mathrm{CD}^{+} 5 \mathrm{R}^{+}\left(p=2.9 \times 10^{-3}\right)$, and between TAC-operated cMy-mOVA + OT-I T cells and cMy-mOVA + OT-I/OT-II T cells: CD3 ${ }^{+}\left(p=5.4 \times 10^{-3}\right), \mathrm{CD}^{+}(p=0.03), \mathrm{CD}^{+}(p=0.11), \mathrm{CD}^{+} 5 \mathrm{R}^{+}(p=0.73)$; Mann-Whitney U-Test. 
In Figure 10B, the separated analysis for animals which received only OT-I T cells and those that received a mixture of OT-I and OT-II T cells is shown. Notably, the myocardium of animals that received only OT-I T cells showed significantly more infiltrated T cells $\left(\mathrm{CD}^{+}, \mathrm{CD} 4^{+}, \mathrm{CD} 8^{+}\right)$after TAC operation compared to cMy-mOVA animals without transfer and those which received both T cell types. Hereby, up to $0.04 \mathrm{CD}^{+}$and $\mathrm{CD}^{+}$and $0.4 \mathrm{CD}^{+}$cells per $\mathrm{mm}^{2}$ myocardium were detected. Average numbers of T cell infiltrates in hearts after OT-I/OT-II transfer were similar to control cMy-mOVA mice. B cell infiltration was similar in TAC-operated cMy-mOVA and those which additionally received OT-I T cells (on average 0.19 and 0.22 cells per $\mathrm{mm}^{2}$ ), whereas after the transfer of OT-I and OT-II T cells, a lower number of $B$ cells was detected in the tissue (in the mean 0.07 cells per $\mathrm{mm}^{2}$ ).

Additionally, leukocyte composition in the spleen and heart of cMy-mOVA mice which received adoptive transfer of OT-I and OT-II T cells was analyzed but revealed no major differences in overall leukocyte proportion in these mice after TAC operation compared to controls (Supplement, Ch. 7.1.1). Moreover, adhesion molecules expression on endothelial cells in the heart was not altered after TAC operation and consequently did not enhance leukocyte infiltration by this mechanisms (Supplement, Ch. 7.1.2).

\subsubsection{Activation of OVA-specific cytotoxic T lymphocytes}

The splenocytes of the mice were used as effector cells in cytotoxicity assays to determine the lysis rate of OVA-expressing (RMA OVA-eGFP) or control RMA (RMA eGFP) cells. For this, splenocytes were restimulated in vitro for 4 days with OVA protein and IL-2. In Figure 11, the specific lysis of the target cells by restimulated splenocytes isolated of cMy-mOVA which received either OT-I T cells or OT-I and OT-II T cell is shown. The lysis of OVA-expressing RMA targets ranged on average from $12.1 \%$ (TAC) to $13 \%$ (sham) at the highest effector to target (E:T) ratio by splenocytes of animals, which received OT-I T cells. Splenocytes of animals that received OT-I and OT-II T cells showed a mean specific lysis of OVA-expressing targets up to $35.6 \%$ (TAC) and $39.4 \%$ (sham). Splenocytes of TAC-operated cMymOVA mice, which did not receive adoptive transfer showed a slightly lower lysis rate of $9 \%$ on the highest E:T ratio (Röhrborn et al., unpublished data; data not shown). Killing of the OVA-expressing RMA cell line, in contrast to the RMA control showed that OVA-specific CTLs were present in spleens of TAC- and sham-operated animals 10 weeks after transfer. Here, the additional transfer of OT-I T cells increased the capability of CTLs to kill the OVA-expressing target line compared to cMy-mOVA mice without transfer. The combined transfer of $\mathrm{CD} 4^{+}$and $\mathrm{CD} 8^{+}$specific for OVA even lead to a higher 
cytotoxic response of CTLs towards OVA. However, no major differences in the target lysis rates occurred between splenocytes obtained from sham- and TAC-operated animals, showing that even without increased afterload, OVA-specific CTLs became activated and were able to lyse OVAexpressing cells.

\section{A}

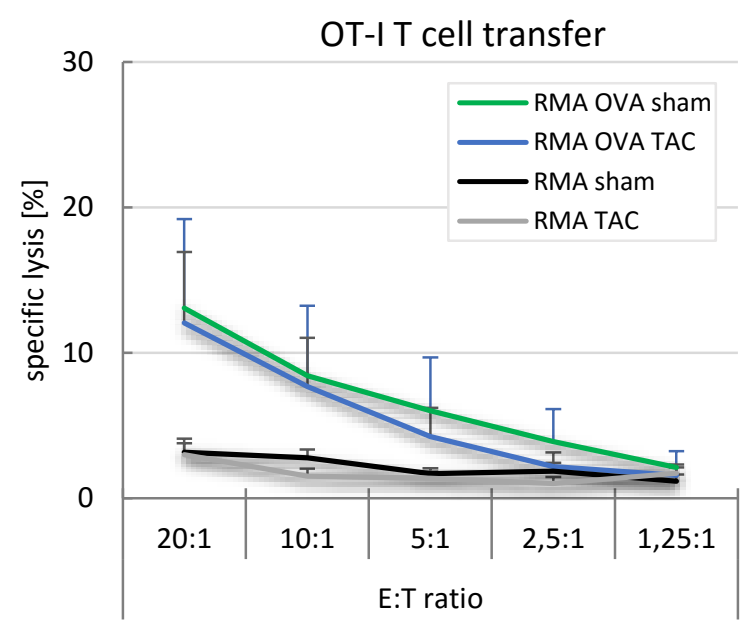

B

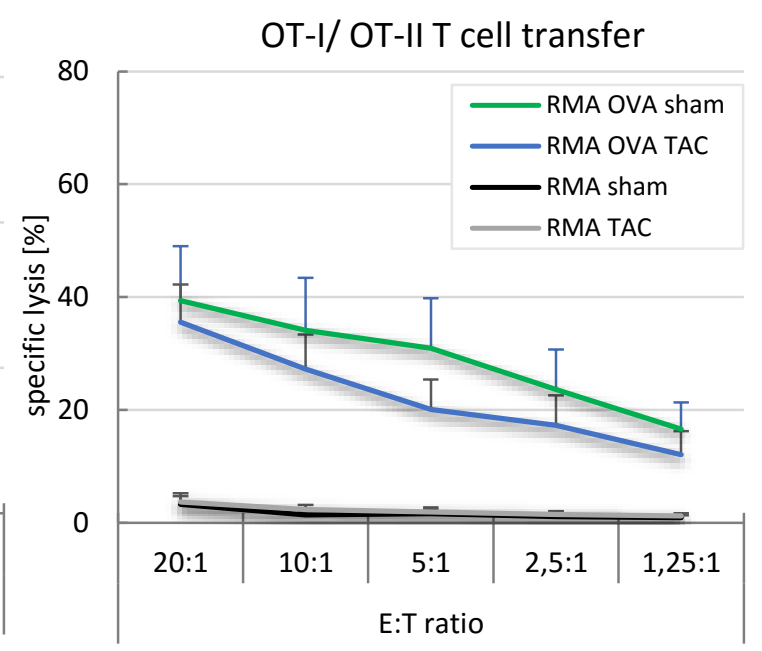

Figure 11 Cytotoxicity assays performed with splenocytes of TAC- or sham-operated mice which received adoptive transfer of either (A) OT-I T cells or (B) a mixture of OT-I/OT-II T cells.

Splenocytes were obtained 10 weeks after transfer of TCR-transgenic T cells into cMy-mOVA mice and were restimulated in vitro with OVA protein and IL-2 for 4 days before used as effector cells in ${ }^{51} \mathrm{Cr}$ release assays. Control RMA (RMA-eGFP) and RMA OVA (RMA eGFP-OVA) served as targets in the test. Shown is the mean of specific lysis + SEM at different E:T ratios. OT-I T cell transfer: sham/TAC ( $n=10$, each); OT-I/OT-II T cell transfer: $\operatorname{sham}(n=11), \operatorname{TAC}(n=14)$.

\subsubsection{Anti-OVA antibody generation}

Whether OVA-specific antibodies were generated after adoptive transfer of OT-I or a mixture of OT-I/OT-II TCR-transgenic T cells was analyzed by ELISAs. For this analysis, pre-sera obtained before operation ( 7 days pre operation) of cMy-mOVA mice and sera, obtained after animals were sacrificed (normally after 10 weeks), were used in serial dilutions to determine the OVA-specific antibody titer compared to serum of control animals which had been immunized with recombinant OVA protein. The OD value of the reference serum was set to $100 \%$ for the lowest dilution (1:30) to calculate the relative anti-OVA antibody level of other sera. As seen in Figure 18, no OVA-specific antibodies arose after the transfer of OVA-specific T cells into cMy-mOVA mice undergoing surgery. The slightly higher anti-OVA titer at the end point of experiments in those animals can be caused by increased age of examined animals as it was also found in untreated controls (data not shown). After OT-I T cell 
transfer, animals displayed a similar OVA-titer independent of the type of surgery (TAC/sham). These results were similar to anti-OVA titers of cMy-mOVA mice, which did not received adoptive transfers (Röhrborn et al., unpublished data; data not shown). Thus, the transfer of TCR-transgenic T cells had no influence on the anti-OVA antibody generation.

Anti-OVA titers after transfer of

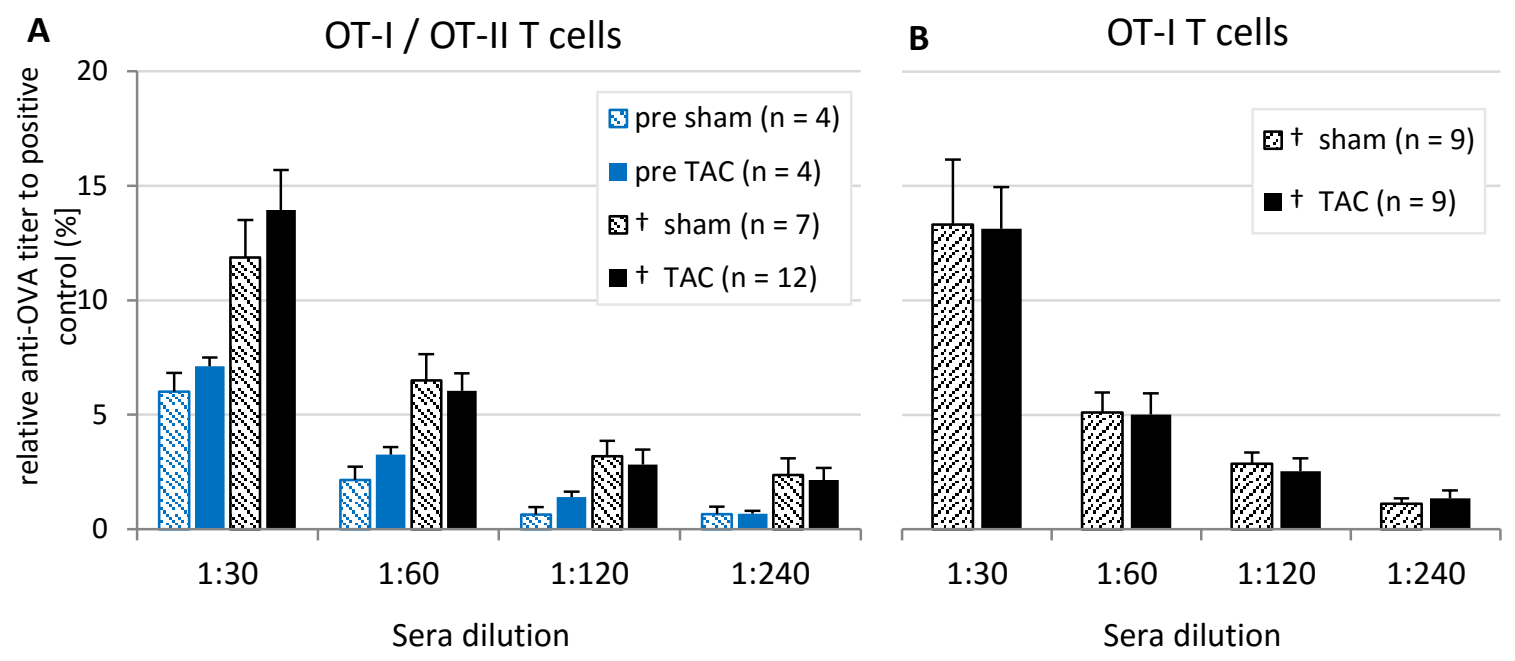

Figure 12 Anti-OVA-antibody titer in CMy-mOVA mice which received (A) OT-I and OT-II or (B) only OT-I $T$ cells before sham and TAC operation.

Sera were taken 1 week prior to surgery (pre) and at the end point of experiments $\left({ }^{\dagger}\right)$. Shown are relative anti-OVA titers + SEM compared to control sera, which were obtained from prior OVA-immunized 129/SV animals.

\subsubsection{Cardiac fibrosis}

The pathology of HF includes cardiac fibrosis. After TAC operations of cMy-mOVA mice, increased fibrosis was detected by histological evaluation of Sirius Red-stained tissue slides. To determine whether the transfer of T cells specific for a cardiac antigen increases fibrosis, myocardia of animals which received adoptive transfers were analyzed. In Figure 13A, histological example pictures of Sirius Red-stained myocardia are depicted which show that the collagen staining is more intense in tissues after TAC operation. The proportion of collagen-stained area to total myocardia area was calculated, showing that the TAC operation and the additional transfer of OT-I and OT-II T cells led to increased fibrosis (18.3\%) compared to TAC-operated cMy-mOVA mice, which did not received T cells (12.5\%), although this reached not statistical significance (Figure 13B). In contrast, the transfer of only OT-I T cells resulted in a comparable level of fibrosis in TAC-operated mice (12.2 \%) to cMy-mOVA mice, 
undergoing the same surgery. Fibrosis in sham-operated animals ranged from $7.1 \%$ (cMy-mOVA + OT-I T cells) to $9.3 \%$ (cMy-mOVA + OT-I/OT-II T cells) and showed no major differences between the animal groups. The only significant changes in fibrosis were detected when TAC- and sham-operated animals were compared. Overall, there might be a trend towards increased fibrosis after TAC, if additional OVA-specific $\mathrm{CD} 4^{+} \mathrm{T}$ cells were transferred, but these changes were not significant in this analysis.
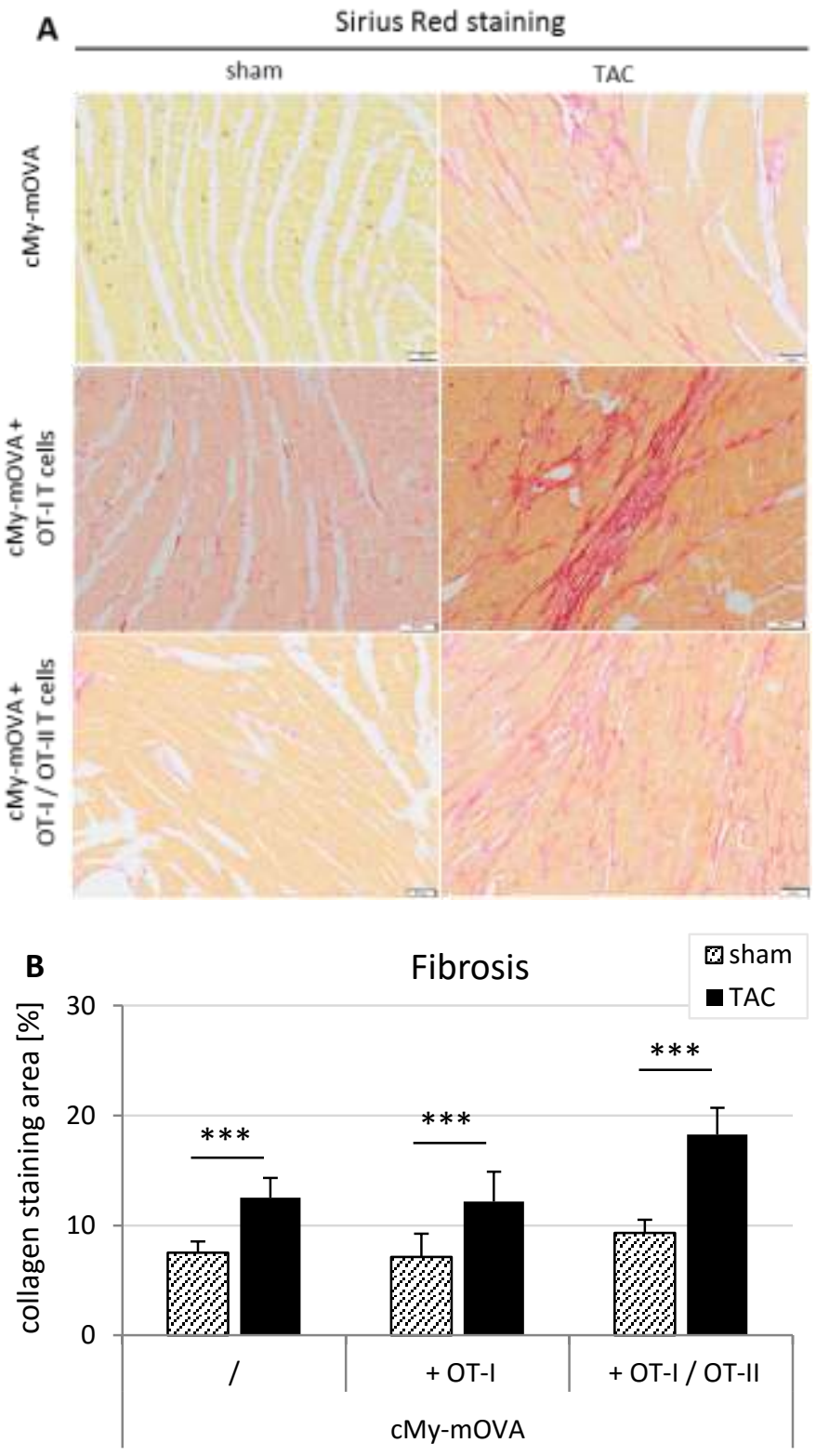

Figure 13 Fibrosis in the myocardia of cMy-mOVA and cMy-mOVA mice which received adoptive transfer of either $0 \mathrm{OT}-\mathrm{I}$ or OT-I and OT-II T cells.

(A) Sirius Red staining of cMy-mOVA myocardia of sham- and TAC-operated mice to visualize fibrosis. Scale bars in each picture (bottom right corner) represent $50 \mu \mathrm{m}$ each. (B) Proportion of fibrotic area per myocardium calculated by cellSens dimension software. CMymOVA: sham $(n=22)$, TAC $(n=15)$; $c M y-$ mOVA + OT-I T cells: sham $(n=8)$, TAC $(n=$ 11); cMy-mOVA + OT-I/OT-II T cells: sham $(n=10)$, TAC $(n=14)$. Differences between cMy-mOVA vs. cMy-mOVA + OT-I T cells: sham $(p=0.61)$, TAC $(p=0.34)$; cMymOVA vs. cMy-mOVA + OT-I/OT-II T cells: sham $(p=0.07)$, TAC $(p=0.21)$; differences between sham- and TAC-operated animals: cMy-mOVA + OT-I T cells:

$\left(p=9.3 \times 10^{-4}\right) ; c M y-m O V A+$ OT-I/OT-II T cells: $\left(p=1.2 \times 10^{-5}\right)$; Mann-Whitney Utest; ${ }^{* * *}<0.001$. 


\subsubsection{Hypertrophy and heart function}

After TAC operation, eliciting pressure overload-induced HF, hearts size and weight were increased in cMy-mOVA mice due to cardiac hypertrophy. To analyze heart weight, which can differ between animals due to their overall phenotype (size, weight, sex of mice), ventricular weight ( $\mathrm{vw}$ ) is generally normalized to the tibia length of animals (Yin et al., 1982). Effects of the transfer of T cells specific for OVA into cMy-mOVA mice on the phenotype of the heart are summarized in Figure 14. The heart weight was not significantly affected by the adoptive transfer of OVA-specific T cells, showing similar heart weight after TAC operations for each animal group, ranging from ratios of 10.3 (+ OT-I T cells) to 12.1 (+ OT-I/OT-II T cells). Compared to sham-operated animals, all groups showed a significant higher heart weight after TAC operation.

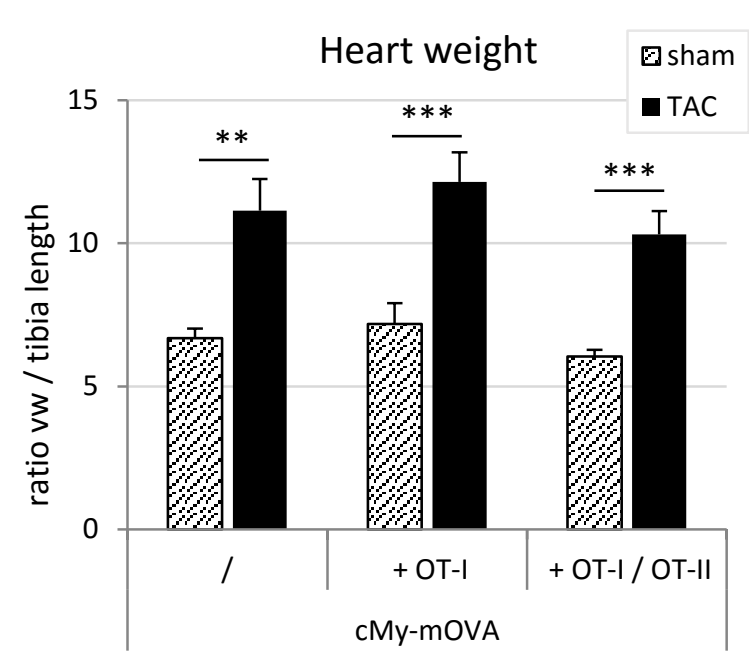

Figure 14 Heart weight of cMy-mOVA mice and mice which received OVA-specific T cells.

Shown are the mean of ventricular weight (vw) [mg] to tibia length [mm] ratios after experimental ending (10 weeks post operation) + SEM. Animal numbers w/o transfer: sham $(n=21)$, TAC $(n=17)$; + OT-I T cells: sham $(n=8)$, TAC $(n=11)$; + OT-I/OTII T cells: sham $(n=8)$, TAC $(n=14)$. Differences between sham- and TAC-operated animals: cMy-mOVA $\left(p=3.6 \times 10^{-3}\right)$, OT-I T cell transfer $\left(p=5.2 \times 10^{-7}\right)$, OT-I/OT-II T cell transfer $(p=5.6 \times$ $\left.10^{-7}\right)$. CMy-mOVA w/o transfer vs. cMy-mOVA + OT-I T cells: sham ( $p=0.36)$, TAC ( $p=0.49)$; cMymOVA w/o transfer vs. cMy-mOVA + OT-I/OT-II T cells: sham: $(p=0.26)$, TAC $(p=0.55)$; MannWhitney U-test.

Major parameters to assess heart function were measured by echocardiography and include ejection fraction (EF), which measures the fraction of blood pumped out of the heart with each heartbeat and the left ventricular fractional area shortening (FAS), which estimates the cardiac contractility. Additionally, hypertrophy parameters including diastolic and systolic anterior wall thickness (AWThd, AWThs) were analyzed and evaluated. After TAC operation, hypertrophy increased in all groups compared to sham-operated animals, whereas the overall function of the heart decreased compared to animals without aortic banding. Comparison of all parameters showed that neither the cardiac hypertrophy (wall thickness) nor functional parameters of the heart were significantly affected by the additional transfer of OVA-specific T cells into cMy-mOVA mice (Figure 21). 

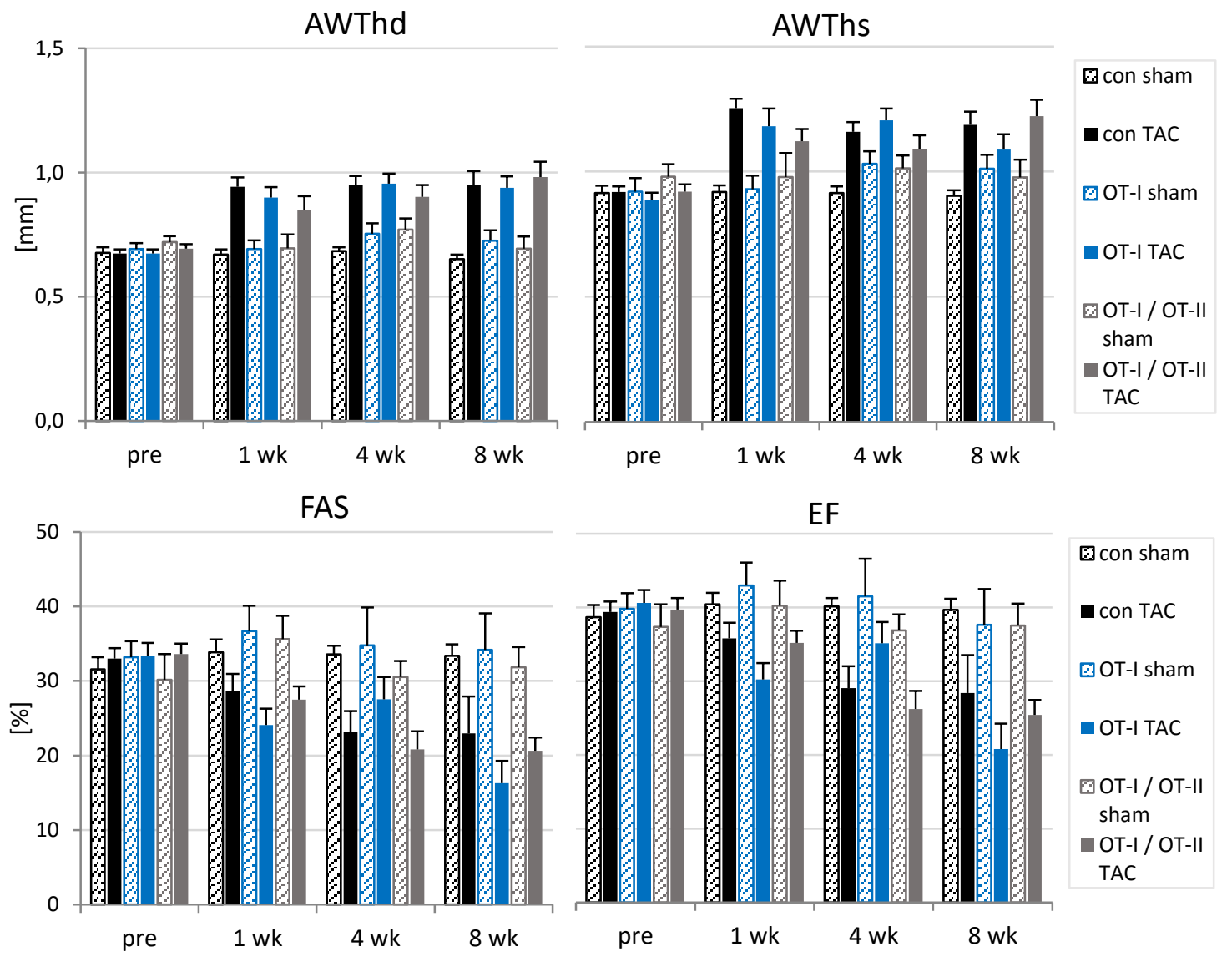

Figure 15 Analysis of hypertrophy and heart function of cMy-mOVA mice by echocardiography.

Mice underwent TAC or sham surgery and received TCR-transgenic OT-I or a mixture of OT-I and OT-II T cells. Over the time of experiments, heart functionality was monitored via echocardiography, including measurements/calculation of ejection fraction (EF), left ventricular area shortening (FAS), diastolic anterior wall thickness (AWThd) and systolic anterior wall thickness (AWThs). Animal numbers: w/o transfer: sham ( $n=21)$, TAC $(n=17)$; + OT-I T cells: sham $(n=8)$, TAC $(n=11)$; + OT-I/OT-II T cells: sham $(n=$ 8), TAC ( $n=14)$. Differences between TAC-operated cMy-mOVA and cMy-mOVA + OT-I T cells at 8 weeks post operation: AWThd $(p=0.86)$, AWThs $(p=0.25), \operatorname{FAS}(p=0.25), \operatorname{EF}(p=0.23)$ and between cMy-mOVA and cMy-mOVA + OT-I/OT-II T cells at 8 weeks post surgery: AWThd $(p=0.72)$, AWThs $(p=0.70)$, FAS $(p$ $=0.63), E F(p=0.56)$, student's t-test. 


\subsubsection{Analysis of immune reactions in cMy-mOVA/OT-II}

To further increase the proportion of autoreactive T cells and to ensure their presence at all time points during the development of HF after TAC, cMy-mOVA mice were bred with OT-I and OT-II mice to create double-transgenic mice, which express OVA exclusively on cardiomyocytes and whose T cells were specific for this cardiac antigen. The attempt to create cMy-mOVA/OT-I mice failed because of early mortality between days 1 to 5 after birth due to unknown reasons. Analysis of the hearts showed no pronounced $\mathrm{T}$ cell infiltration, however impairment of heart function by only low numbers of autoreactive CTLS at this time point cannot be excluded.

In contrast, cMy-mOVA/OT-II mice were successfully bred and were therefore available for further analysis. Mice were TAC- and sham-operated and monitored for up to 10 weeks. After experimental ending 10 weeks post operation, splenocytes of cMy-mOVA/OT-II mice were used for flow cytometric analysis to determine proportions of lymphocyte populations and the expression of activation markers, including CD25 and CD69 which can be found on activated T cells (Figure 16). Half of the splenocyte population consisted of B cells (CD45R ${ }^{+}: 53.9 \%$ (TAC) to $54.1 \%$ (sham)), followed by T helper cells ( $\mathrm{CD}^{+} \mathrm{CD}^{+}: 20.6 \%$ (TAC) to $23.0 \%$ (sham)). Significantly smaller numbers of cytotoxic T cells (CD3 ${ }^{+} \mathrm{CD}^{+}: 4.8 \%(\mathrm{TAC})$ to $6.0 \%$ (sham); $\left.\mathrm{p}=0.04\right)$ than in wildtype mice were found whereas the proportion of $\gamma \delta$ T cells was in the normal range. Numbers of NK cells were significantly higher after TAC operation (DX5 ${ }^{+} \mathrm{CD} 3: 4.3 \%$ (sham) to $5.3 \%$ (TAC); $\mathrm{p}=2.1 \times 10^{-3}$ ) compared to controls. Additionally, the numbers of TCR V $\beta$ 5.1/5.2 positive $\mathrm{CD}^{+}$and $\mathrm{CD} 8^{+}$cells were determined, showing a significantly lower number of those cells after TAC operation $\left(p=6.2 \times 10^{-3}\right)$. Importantly, these data demonstrated that the majority of T cells carried the transgenic TCR. Further analysis of activation marker on $\mathrm{CD}^{+}$cells revealed that after TAC operation, significantly higher numbers of $\mathrm{CD} 4^{+} \mathrm{CD} 25^{+}$ cells were present in the splenocyte population $\left(p=7.5 \times 10^{-3}\right)$. However, examination of CD69+ cells showed no major differences in the activation status of T helper and cytotoxic T cells between shamand TAC-operated animals. 

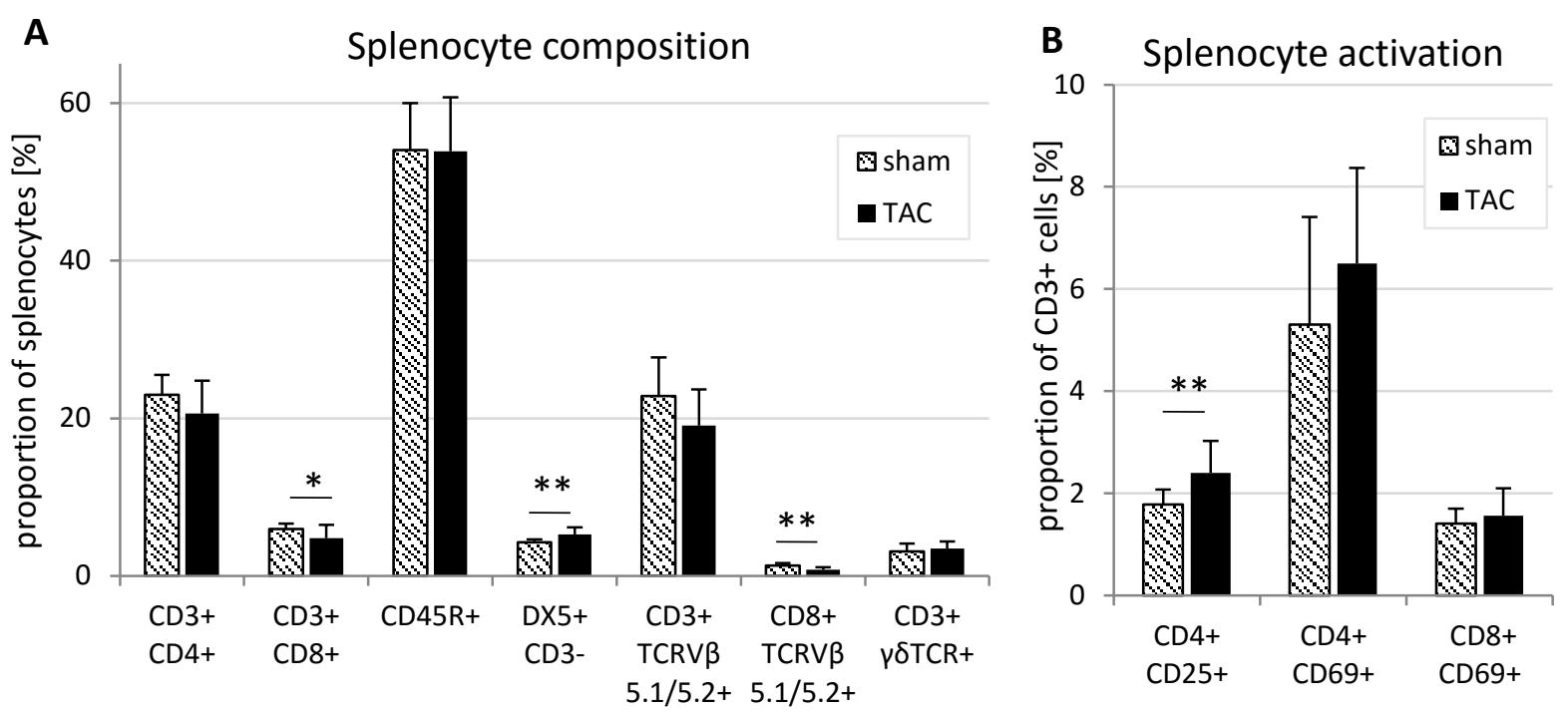

Figure 16 Analysis of surface markers on splenocytes, isolated after experimental ending of cMymOVA/OT-II mice which underwent TAC or sham surgery.

Shown is the cell proportion of (A) total splenocytes or (B) $\mathrm{CD3}^{+}$cells + SEM. Animal numbers: sham $(n=10)$, TAC $(n=17)$. TAC $(n=17)$, sham $(n=10)$, mean duration of experiments: TAC (67 days), sham (69 days). ${ }^{*}<0.05$; ${ }^{*}<0.01$; student's t-test. (A) Staining for various cell types including $\mathrm{T}$ helper cells $\left(\mathrm{CD} 3^{+} \mathrm{CD} 4^{+}\right)$, cytotoxic T cells $\left(\mathrm{CD}^{+} \mathrm{CD} 8^{+}\right)$, B cells $\left(\mathrm{CD} 45 \mathrm{R}^{+}\right)$, NK cells $\left(\mathrm{DX} 5^{+} \mathrm{CD} 3^{-}\right)$, and for TCRs on $\mathrm{CD}^{+}$and $\mathrm{CD} 8^{+}$cells. (B) Staining for activation marker CD25 and CD69 on T helper and cytotoxic T cells.

Furthermore, intracellular cytokine staining (ICS), that detect cytokine production by immune cells in combination with cell surface makers, was performed by flow cytometry to monitor whether the CD4 ${ }^{+}$ T cells exhibit a polarization towards a certain cytokine profile. Here, the proportion of T helper cells expressing cytokines, including various interleukins (IL-2, IL-4, IL-6, IL-10, IL-17A, IL-17B), interferon gamma (IFN $\gamma$ ), tumor necrosis factor $\alpha$ (TNF $\alpha$ ), transforming growth factor $\beta$ (TGF $\beta$ ) and additionally the amount of regulatory T cells (Tregs) was determined in splenocytes 10 weeks post operations (Figure 17). Tregs, which are known to be important for immune modulation and tolerance to selfantigens, made up only $7.8 \%$ of total $\mathrm{CD}^{+}$cells in TAC-operated and $10.9 \%$ of $\mathrm{CD}^{+}$cells in shamoperated animals. CD4 ${ }^{+}$T cells expressing TNF $\alpha$ and TGF $\beta$ were most abundant. The proportion of $\mathrm{TNF}^{+} \mathrm{CD}^{+} \mathrm{T}$ cells was significantly higher in TAC-operated animals (8.34 \%) than sham-operated (4.1\%) cMy-mOVA/OT-II mice. In contrast, a greater proportion of TGF $\beta^{+} \mathrm{CD}_{4}{ }^{+}$cells was found after sham operation (8.9\%), however the difference to TAC-operated animals (5.4\%) was not significant. A significantly higher proportion of IL- $17 \mathrm{~A}^{+} \mathrm{CD} 4^{+} \mathrm{T}$ cells (4.1\%) was found in TAC-operated than shamoperated animals. IL-2 ${ }^{+} \mathrm{CD} 4^{+}$and $\mathrm{IL}-10^{+} \mathrm{CD} 4^{+} \mathrm{T}$ cells were equal for both animal groups (IL-2 ${ }^{+}: 1.7 \% \mathrm{TAC}$, $1.9 \%$ sham; IL-10 after TAC operation (IL-4+: $0.4 \%$, IL-6+: $1.1 \%$ ) although at low level. Additionally, a significantly higher 
amount of IFN $\gamma^{+} C D 4^{+}$cells were found after TAC operation (sham: $0.1 \%$; TAC: $2.7 \%$ ). Generally the proportion of $\mathrm{T}$ helper cells with an inflammatory $\mathrm{Th}_{1}$ and a $\mathrm{Th}_{17}$ cytokine profile was increased at 10 weeks post TAC operation compared to sham-operated cMy-mOVA/OT-II mice.

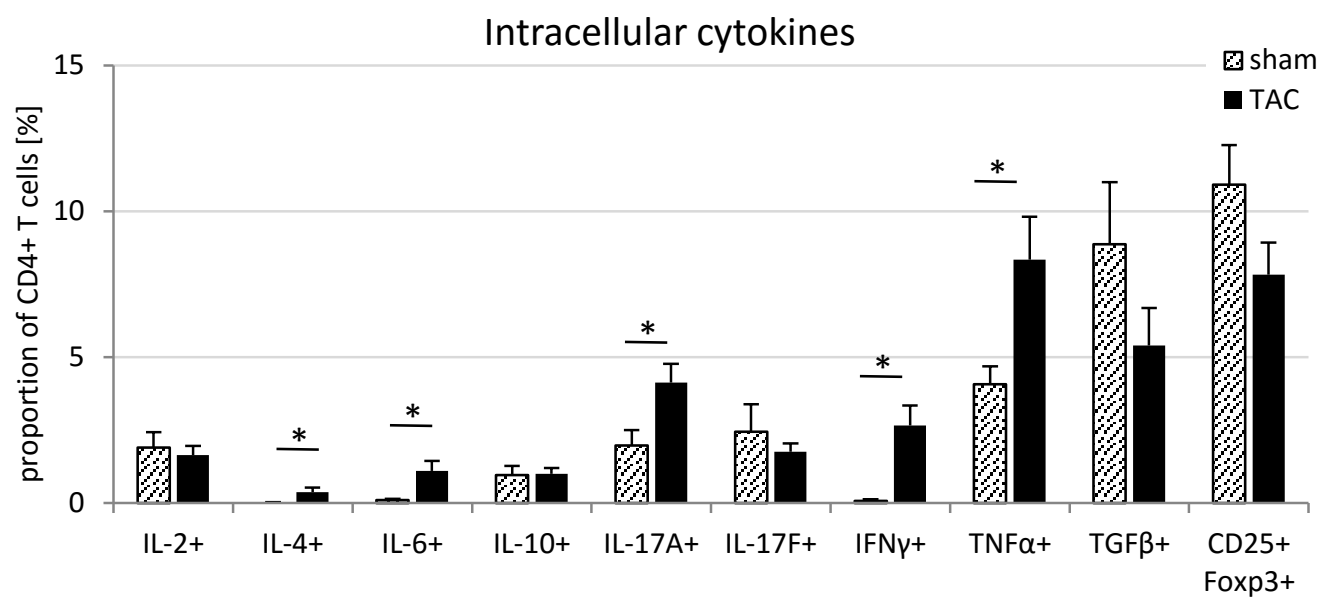

Figure 17 Proportion of $\mathrm{CD}^{+} \mathrm{T}$ cells of cMy-mOVA/OT-II mice expressing intracellular cytokines after TAC and sham operations.

Splenocytes were analyzed 10 weeks post operations via flow cytometry. Shown is the mean proportion for various intracellular cytokines of CD4 $4^{+}$cells + SEM. Sham $(n=10)$, TAC $(n=17)$. Significant differences of cytokines levels between sham- and TAC-operated animals are marked with asterisks, showing an increased proportion of Th 1- $_{1}$ and Th17-polarized T helper cells: IL-2 $(p=0.67), I L-4(p=0.045)$, IL-6 $(p=7.6$ $\left.x 10^{-3}\right)$, IL-10 $(p=0.84)$, IL-17A $(p=0.017)$, IL-17F $(p=0.51)$, TNF $\alpha(p=0.016)$, IFNY $\left(p=1.6 \times 10^{-3}\right)$, TGF $\beta(p$ $=0.18)$, FoxP3/CD25 $(p=0.09)$.

\subsubsection{Anti-OVA antibody generation}

To test whether OVA-specific antibodies arose in cMy-mOVA/OT-II mice, sera of various time points over the course of experiments (pre, 1 week, 4 weeks, 8 weeks) and after experimental ending $\left({ }^{\dagger}\right)$ were collected and analyzed via ELISA tests. The reactivity of sera from sham- and TAC-operated mice against OVA were determined at several dilutions compared to control sera of mice immunized against OVA. As seen in Figure 18, the level of OVA-specific antibodies in cMy-mOVA/OT-II mice was not increased after TAC operation, showing similar levels of anti-OVA titers compared to cMy-mOVA mice (Röhrborn et al., unpublished data) and to cMy-mOVA mice which additionally received T cell transfer (Ch. 4.1.1.3). 


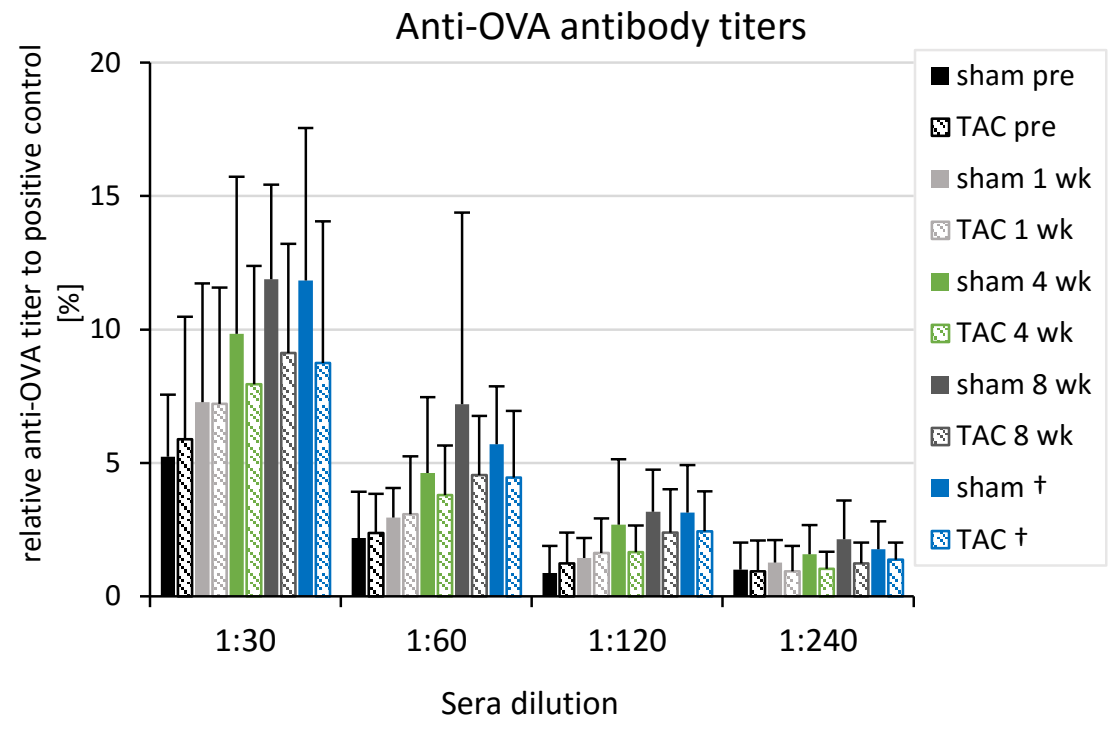

Figure 18 Anti-OVA antibody titers in sera of cMy-mOVA/OT-II mice obtained at different time point pre and post sham and TAC operation.

Shown are relative anti-OVA titers compared to sera of OVA-immunized 129/Sv mice + SEM. Sham $(n=$ $15), \operatorname{TAC}(n=22)$.

\subsubsection{Infiltration of lymphocytes into heart tissue}

To analyze the infiltration of immune cells into myocardia of cMy-mOVA/OT-II mice, sections of paraffin embedded specimens were used for histological analysis. Representative IHC stainings in Figure 19A show the presence of $\mathrm{CD}^{+}, \mathrm{CD}^{+}, \mathrm{CD}^{+}$and $\mathrm{CD}^{2} 5 \mathrm{R}^{+}$cells in the myocardium of TACoperated cMy-mOVA/OT-II mice. Number of infiltrating cells were calculated via cellSens dimension software and are depicted in Figure 19B. The most abundant cells found in the myocardia of cMymOVA/OT-II were CD45R ${ }^{+}$cells (0.03 (sham) to 0.05 (TAC) cells $/ \mathrm{mm}^{2}$ ) and CD8 ${ }^{+}$T cells $\left(0.01\right.$ cells $/ \mathrm{mm}^{2}$ ) in TAC-operated animals, but generally lower numbers of these cells were found than in myocardia of cMy-mOVA mice. The amount of $\mathrm{CD}^{+}$cells in cMy-mOVA/OT-II mice was higher after sham operation ( 0.01 cells $/ \mathrm{mm}^{2}$ ) than in TAC-operated animals $\left(7.1 \times 10^{-3}\right.$ cells $\left./ \mathrm{mm}^{2}\right)$, and overall more $\mathrm{CD}^{+}$cells were found in cMy-mOVA/OT-II mice than in cMy-mOVA mice. The amount of CD4 ${ }^{+}$cells in myocardia of cMy-mOVA/OT-II mice was significantly higher than in cMy-mOVA mice. In summary, cMymOVA/OT-II animals show lower numbers of infiltrated $\mathrm{CD} 8^{+}$and $\mathrm{CD} 45 \mathrm{R}^{+}$cells, but higher $\mathrm{CD} 4^{+}$and $\mathrm{CD}^{+}$cell numbers than cMy-mOVA mice after TAC operation. 
A CMy-mOVA/OT-II (TAC)
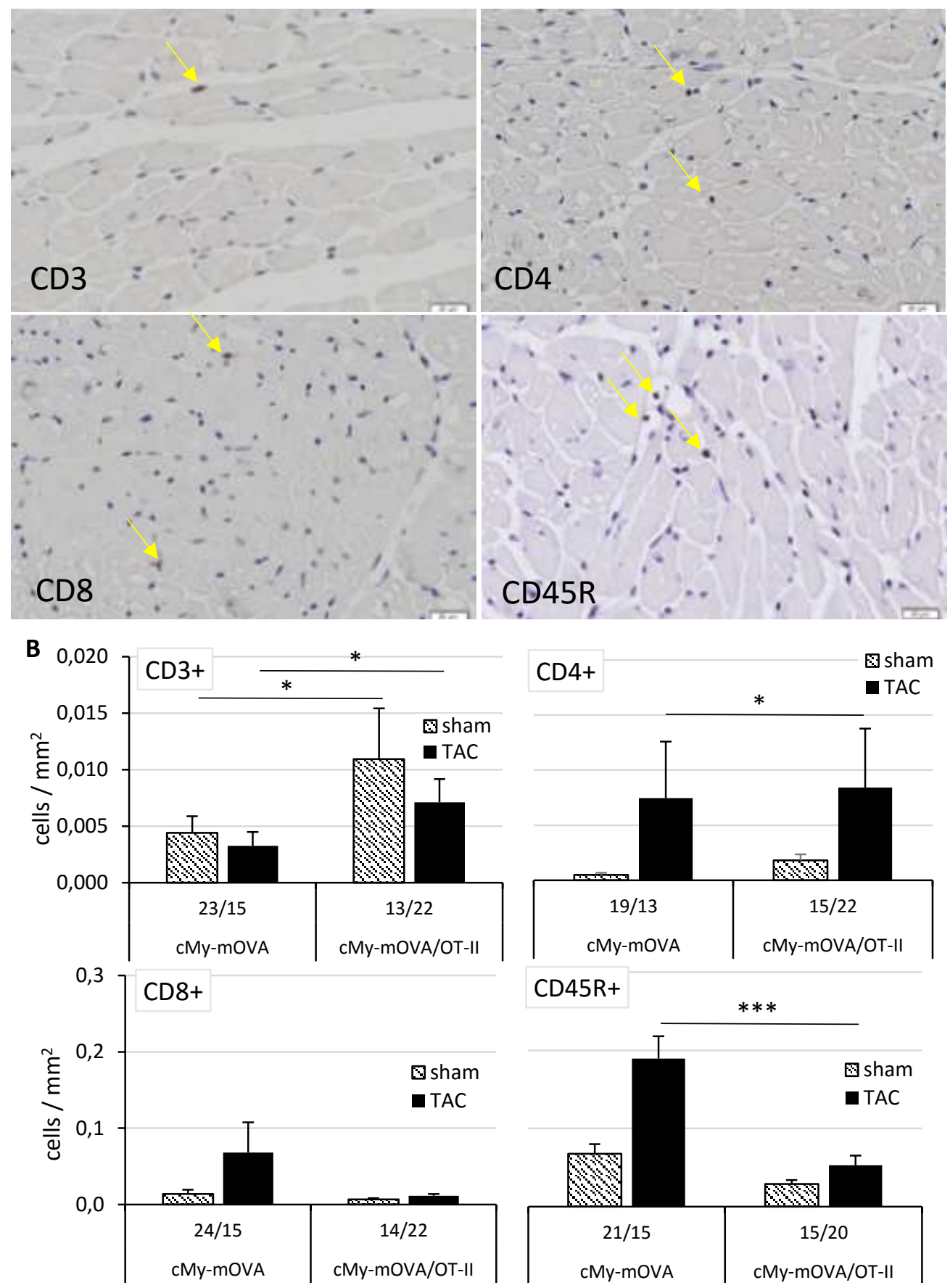

Figure $19 \mathrm{IHC}$ analysis of infiltrating cells into the myocardia of $\mathrm{cMy}$-mOVA/OT-II animals.

(A) Example pictures of IHC stained tissue slides of myocardia from cMy-mOVA/OT-II mice after TAC operation. Positive stained cells indicate infiltrating lymphocytes and are marked with asterisk. Scale bars in each picture (bottom right corner) represent $20 \mu \mathrm{m}$ each. (B) Calculated lymphocyte numbers per defined area in myocardia of cMy-mOVA/OT-Il mice compared to control cMy-mOVA, all of which underwent TAC or sham surgery. Shown are the mean cell numbers per $\mathrm{mm}^{2}+\mathrm{SEM}$; Animal numbers are depicted below each column. Differences between TAC-operated cMy-mOVA and cMy-mOVA/OT-II: CD3 ${ }^{+}$ $(p=0.03), C D^{+}(p=0.02), C D^{+}(p=0.73), \operatorname{CD}^{+} 5 R^{+}\left(p=7.2 \times 10^{-5}\right)$; Mann-Whitney U-test. ${ }^{*}<0.05, * * *<$ 0.001 . 


\subsubsection{Cardiac fibrosis}

Analysis of fibrosis showed that after TAC operation the amount of collagen in the myocardium detected by Sirius Red staining on histological tissue slides was significantly increased in all animal groups compared to sham-operated mice (Figure 20A). In comparison to cMy-mOVA mice, proportion of fibrosis in cMy-mOVA/OT-II mice was slightly lower (6.5 \% (sham) to $10.5 \%$ (TAC)), but was not significantly altered compared to control animals (Figure 20B).
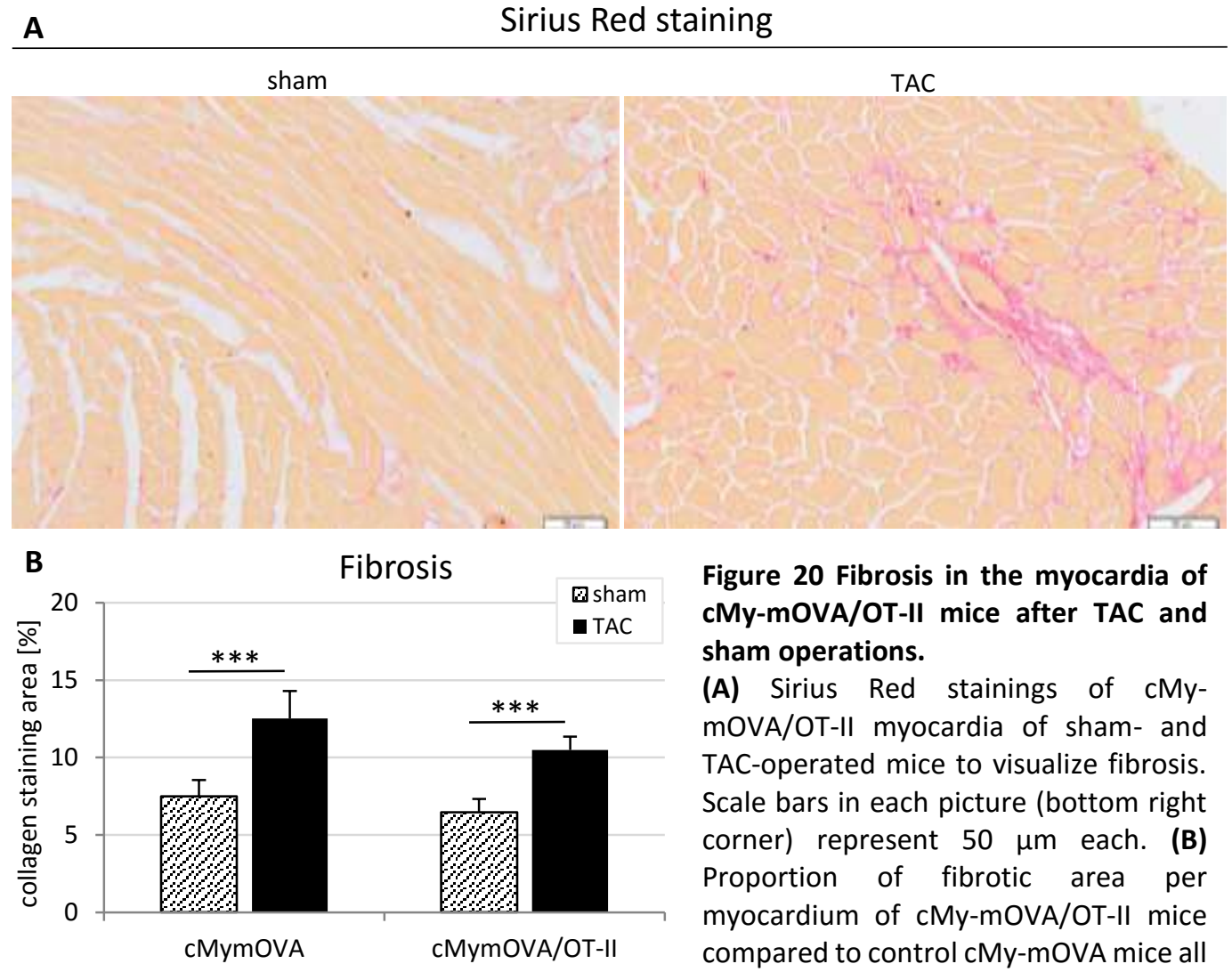

Figure 20 Fibrosis in the myocardia of cMy-mOVA/OT-II mice after TAC and sham operations.

(A) Sirius Red stainings of $\mathrm{cMy}$ mOVA/OT-II myocardia of sham- and TAC-operated mice to visualize fibrosis. Scale bars in each picture (bottom right corner) represent $50 \mu \mathrm{m}$ each. (B) Proportion of fibrotic area per myocardium of cMy-mOVA/OT-II mice compared to control cMy-mOVA mice all of which underwent TAC and sham

surgery. Calculations were performed via cellSens dimension software. CMy-mOVA: sham ( $\mathrm{n}=$ 22), TAC ( $n=15)$; cMy-mOVA/OT-II: sham $(n=15)$, TAC $(n=19)$; cMy-mOVA vs cMy-mOVA/OTII: TAC $(p=0.07)$, sham: $(p=0.27)$; Mann-Whitney U-test, $* * *<0.001$. 


\subsubsection{Hypertrophy and heart function}

The hypertrophy of hearts of cMy-mOVA/OT-II mice after TAC operation was analyzed to detect potential differences to cMy-mOVA mice and those, which additionally received adoptive transfer. In Figure 21, heart weight of TAC- and sham-operated animals after experimental ending is shown. Noticeable, TAC-operated mice of all groups showed a higher heart weight ratio, ranging between 11 to 12 compared to sham animals (6 to 7). CMy-mOVA/OT-II mice displayed a slightly higher heart weight (12.2) compared cMy-mOVA (11.2) or those which received adoptive transfer (11.0) but these changes were not significant. Consequently, a high numbers of $\mathrm{CD}^{+} \mathrm{T}$ cells specific for a cardiac antigen does not appear to have a significant influence on cardiac hypertrophy.

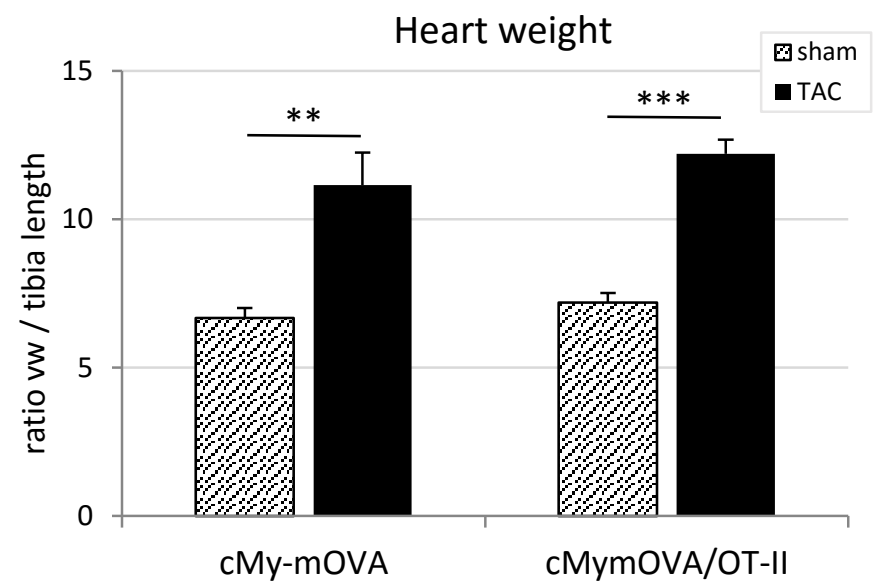

Figure 21 Hypertrophy of TAC- and shamoperated cMy-mOVA and cMy-mOVA/OT-II. Shown are mean ratios of ventricular weight (vw) [mg] to tibia length [mm] + SEM after experimental ending. Animal numbers: cMy-mOVA: sham $(n=15)$, TAC $(n=19)$; cMy-mOVA/OT-II: sham $(n=15)$, TAC ( $n=$ 22). Heart weight was significantly affected by TAC operation: cMy-mOVA $(p=3.6 \times 10$ $\left.{ }^{3}\right)$, cMy-mOVA/OT-II $\left(p=7.4 \times 10^{-7}\right)$. No significant differences were monitored between $\mathrm{cMy}$-mOVA and cMy-mOVA/OT-II mice: sham $(p=0.29)$, TAC $(p=0.39)$.

Next, functional parameters of hearts of cMy-mOVA/OT-II mice after sham and TAC operation were analyzed to clarify whether animals with high frequency of T cells specific for a cardiac antigen are at higher risk for rapid progression into HF after TAC operation compared to cMy-mOVA mice. As seen in Figure 22, the anterior wall thickness in diastole and systole were increased after TAC operation, but was not differing between cMy-mOVA and cMy-mOVA/OT-II mice. In contrast, EF and FAS showed a significantly reduction in cMy-mOVA mice, showing that high numbers of transgenic $T$ cells have influence on the progression of HF. 

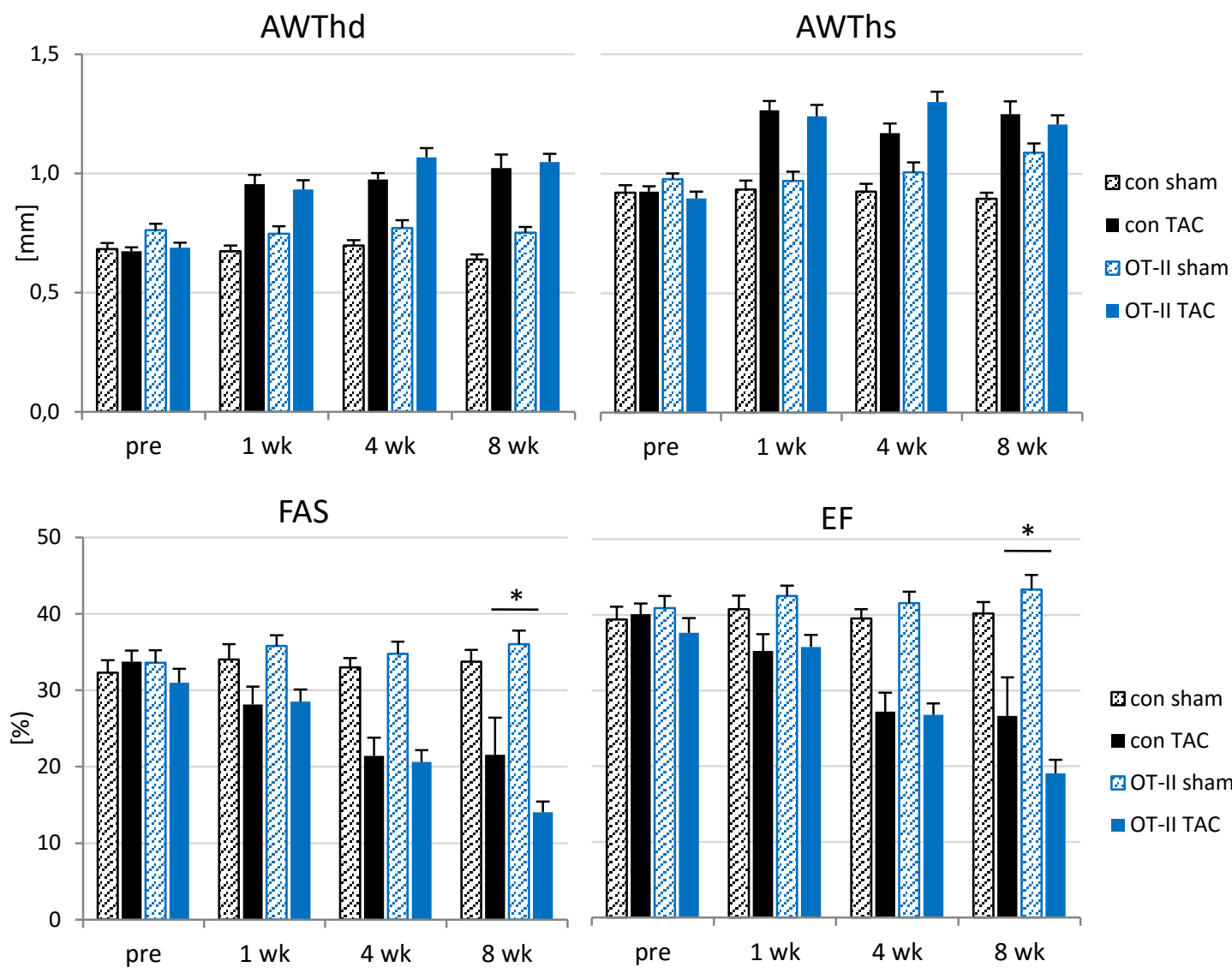

중 con sham

- con TAC

四T-II sham

- OT-II TAC

Figure 22 Analysis of functional parameters assessing heart function of cMy-mOVA/OT-II after TAC and sham operations compared to control cMy-mOVA mice.

Shown are mean of AWThd, AWThs, FAS and EF + SEM determined by echocardiography over the course of experiments. CMy-mOVA: sham $(n=15)$, TAC $(n=19)$; cMy-mOVA/OT-II: sham $(n=15)$, TAC $(n=22)$. Differences at 8 weeks post operation between TAC-operated cMy-mOVA and cMy-mOVA/OT-II animals: AWThd $(p=0.14)$, AWThs $(p=0.79)$, FAS $(p=0.03)$, EF $(p=0.04)$; student's t-test.

\subsubsection{Summary}

The transfer of OVA-specific T cells did not significantly affect cardiac hypertrophy and did not led to deterioration of functional parameters determined by echocardiography. Thus, the transfer of T cells with a specificity for an antigen in cardiomyocytes alone was not sufficient to accelerate the progression to HF. In contrast, the presence of $\mathrm{CD}^{+} \mathrm{T}$ cells, specific for a cardiomyocyte antigen, in high frequency as in cMy-mOVA/OT-II mice was associated with a systemic inflammatory T cell response and a faster progression to $\mathrm{HF}$ at 10 weeks after TAC, indicating that autoreactive $\mathrm{T}$ helper cells can promote progression from hypertrophy to HF and this independent of an antibody response. 


\subsection{Immunogenicity of pluripotent stem cells}

Pluripotent stem cells (PSCs) are seen as important tool for future medical therapies and are therefore an important topic of research. Previous work in our group focused on the in vitro immunogenicity of murine PSC lines including ESCs, iPSCs and maGSCs. It was shown that PSCs exhibit an immunosuppressive phenotype in vitro, because they were able to inhibit antigen-dependent T cell proliferation of $\mathrm{CD}^{+}$and $\mathrm{CD}^{+} \mathrm{T}$ cells in direct co-culture experiments. They were also immunoprivileged because they failed to process and present antigens on MHC class I molecules. However, important for future transplantation therapies is the clarification of the immunogenicity of stem cells and stem cell-derived grafts in vivo. Specifically, the role of minor histocompatibility (miHC) antigens need to be clarified since they cannot be matched in allogeneic transplantations. For this, OVA as a model of a miHC antigen was introduced into several stem cells lines and these were examined in comparison to their wildtype counterparts in otherwise syngeneic recipients. One newly generated iPSC (129/Sv TD11.1; XY) and one new ESC line (BTL1; XY) from 129/Sv mice were used for these experiments and a well-established ESC line (MPI-II; XY) from the same mouse line was included for comparison. The PSCs were transfected to express an OVA-EGFP fusion protein. Four or five of the new cell lines and two clones of the MPI-Il cells were selected for further experiments. All stem cells lines and clones that were used for in vivo analysis had been previously analyzed in vitro for their

pluripotency in terms of OCT4, NANOG, and SSEA-4 expression, expression of the transgene and absence of microbial contaminations via quantitative real-time PCR (qPCR), western blot and flow cytometric analysis (data not shown). 


\subsubsection{Tumor formation in immunodeficient hosts}

To determine their pluripotency in vivo, the cell lines and clones were injected into immunodeficient mice. Stem cells were subcutaneously injected in numbers of $1.5 \times 10^{6}$ cells per animal into various immunodeficient hosts, including $\mathrm{RAG}^{-\%} \mathrm{\gamma c}^{--}$, RAG2 ${ }^{-\%}$, SCID/beige and SCID mice, mainly depending on availability of the mice. All of these animals are mutant mice, lacking B and T cells, however $\mathrm{RAG}^{-/} \gamma \mathrm{C}^{-/-}$and SCID/beige mice are additionally deficient for NK cells or are impaired in their NK cell function, respectively. Initially, two ES wildtype stem cell lines (ESC BTL1, ESC MPI-II), seven OVAexpressing ES cell lines (BTL1 OVA\#1, BTL1 OVA\#4; BTL1 OVA\#9, BTL1 OVA\#14, BTL1 OVA\#20, MPI-II OVA\#1, MPI-II OVA\#4), one iPSC wildtype cell line (iPSC 129/SV TD11.1) and four OVA-expressing iPS cell lines (iPSC 129/Sv OVA\#6, iPSC 129/Sv OVA\#13, iPSC 129/Sv OVA\#18, iPSC 129/Sv OVA\#24) were used for inoculation into immunodeficient mice. In Table 13, the tumor frequency of the various stem cell lines is shown, which ranged from 0 to $100 \%$. The ESC BTL1 OVA cell clones \#9 and \#14 and ESC MPI-II OVA clone \#1 were excluded from most further experiments due to the lack of tumor formation (0 \% each, Table 13). Additionally, some cell lines, like ESC BTL1 OVA\#1 and \#20 show only a minor rate of tumor formation ( $<30 \%$ ) and were also excluded or are reported separately. The failure of some OVA-expressing clones to form teratomas is most likely a random clonal effect and not result of OVA expression. Thus, one OVA-expressing cell clone for each wildtype ESC stem cell line was used for further analysis.

Table 13 Tumor formation in immunodeficient hosts. Cell lines which were further analyzed are marked bold.

\begin{tabular}{|l|l|l|}
\hline Stem cell line & tumor frequency [\%] & $\mathrm{n}$ \\
\hline ESC BTL1 wt & 75 & 12 \\
\hline ESC BTL1 OVA\#1 & 22 & 9 \\
\hline ESC BTL1 OVA\#4 & 64 & 11 \\
\hline ESC BTL1 OVA\#9 & 0 & 3 \\
\hline ESC BTL1 OVA\#14 & 0 & 4 \\
\hline ESC BTL1 OVA\#20 & 29 & 7 \\
\hline ESC MPI-II wt & 90 & 30 \\
\hline ESC MPI-II OVA\#1 & 0 & 11 \\
\hline ESC MPI-II OVA\#4 & 88 & 8 \\
\hline iPSCs 129 SV TD 11.1 & 100 & 17 \\
\hline iPSCs 129 SV TD11.1 OVA\#6 & 100 & 29 \\
\hline iPSCs 129 SV TD11.1 OVA\#13 & 100 & 4 \\
\hline iPSCs 129 SV TD11.1 OVA\#18 & 64 & 11 \\
\hline iPSCs 129 SV TD11.1 OVA\#24 & 89 & 9 \\
\hline total & & 165 \\
\hline
\end{tabular}


The stem cell lines which showed a high proportion of tumor formation in the immunodeficient hosts, thereby demonstrating proliferation and differentiation capacity in vivo, were used for further analysis. In Figure 23, a summary of wildtype and OVA-expressing stem cells is depicted, which shows that all types of cells, comprising ES and iPS and wildtype and OVA-expressing cells lines, were able to form tumors in vivo. Therefore, the expression of OVA appears to have no principal effect on the tumor formation in immunodeficient hosts.

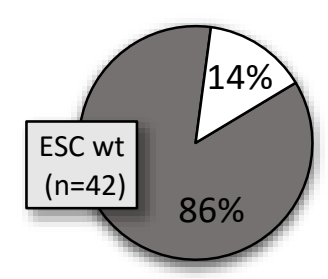

$\square$ w tumor $\square$ w/otumor

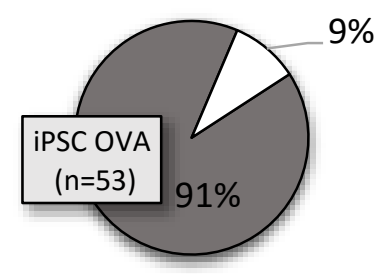

$\square$ w tumor $\square$ w/otumor

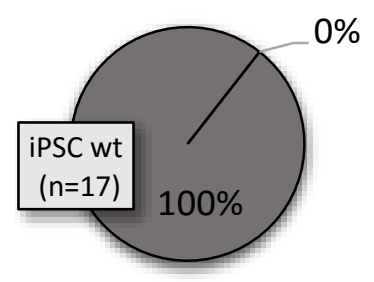

$\square$ w tumor $\square$ w/otumor

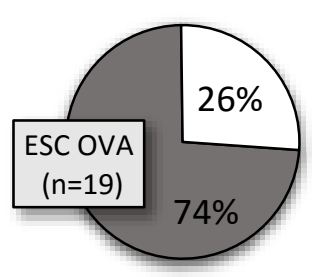

$\square$ w tumor $\square$ w/o tumor

Figure 23 Tumor formation in immunodeficient hosts.

Stem cell lines which were generally not able to give rise to tumors in vivo were already excluded from this summary. Numbers of performed experiments are depicted in the respective circle graph.

\subsubsection{Effects of OVA expression on tumor formation, size and weight in immunodeficient hosts}

The following data were statistically analyzed with respect to tumor formation rate, tumor size, tumor weight and influences of the presence of NK cells, sex of the recipients and type of stem cells on these parameters. Moreover, the duration until experiments were terminated, either due to tumor growth or due to achievement of the end point of experiments (usually 3 month) were compared. Comparison of tumor formation between wildtype and OVA-expressing stem cells showed that the expression of OVA does not lead to an altered tumor formation rate compared to wildtype stem cells (ESC wt vs. ESC OVA: $p=0.19$; iPSC wt vs. iPSC OVA: $p=0.29$; PSC wt vs. PSC OVA: $p=0.21$; Mann-Whitney U-test; Figure 24A). Statistical analysis of teratoma formation between ESC- and iPSC-derived tumors showed no significant differences, neither for tumors derived from wildtype stem cells nor from OVA- 
expressing stem cells (ESC wt vs. iPSC wt: $p=0.36$; ESC OVA vs. iPSC OVA: $p=0.4$, Mann-Whitney Utest; Figure 24A). Further analysis showed that the presence of NK cells in RAG2 ${ }^{-\%}$ and SCID mice had no influence on the tumor formation rate (data not shown) and that tumor formation also showed no statistical differences between the various hosts (RAG2 ${ }^{-1} \gamma \mathrm{Cc}^{-/}, \mathrm{RAG}^{-1}, \mathrm{SCID} /$ beige, SCID, data not shown). The different sex of host animals ( $m$ vs. $f$ ) had also no impact on the tumor formation rate (data not shown). Tumor size and weight were also not influenced by the different type of stem cells (ESC vs. iPSC), or transgene expression (OVA vs. wt) (Figure 24B, 2C).
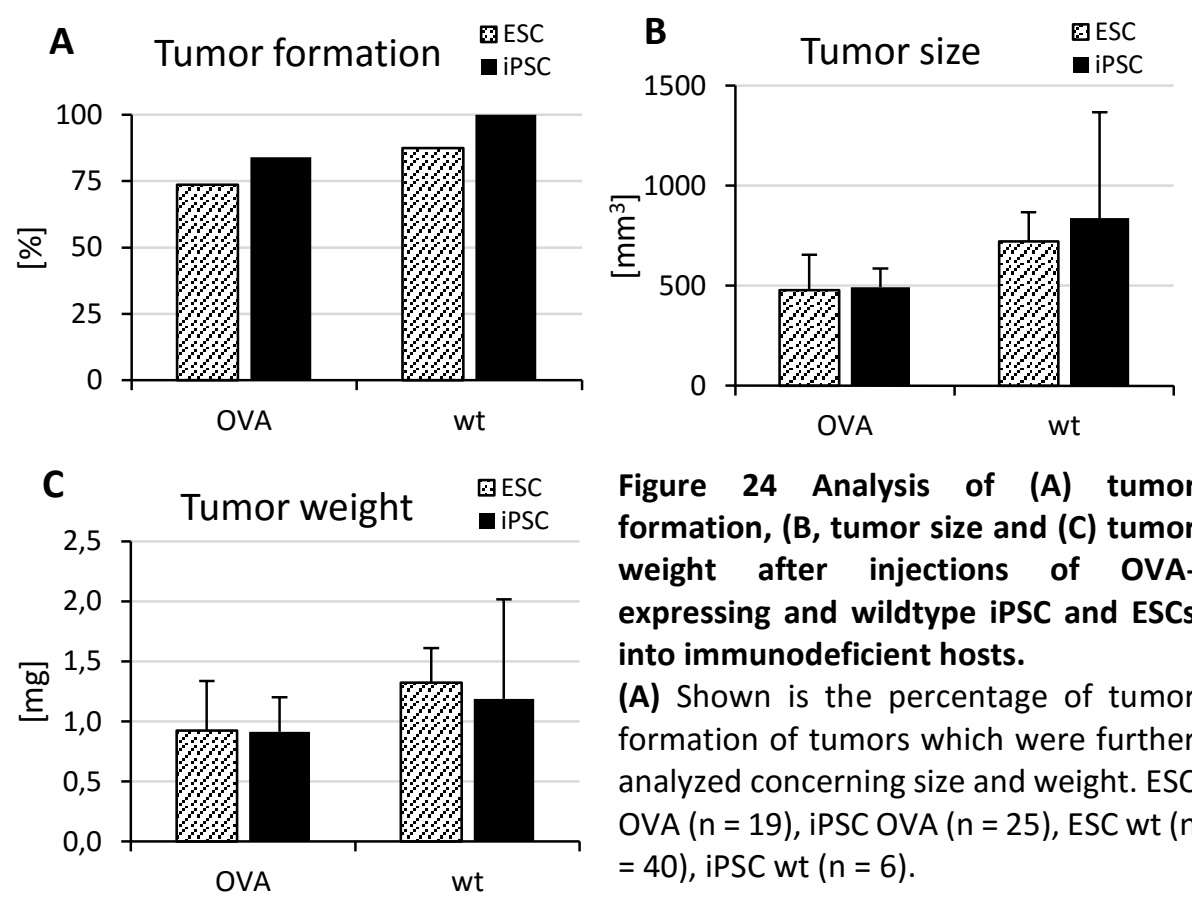

Figure 24 Analysis of (A) tumor formation, (B, tumor size and (C) tumor weight after injections of OVAexpressing and wildtype iPSC and ESCs into immunodeficient hosts.

(A) Shown is the percentage of tumor formation of tumors which were further analyzed concerning size and weight. ESC OVA $(n=19)$, iPSC OVA $(n=25)$, ESC wt ( $n$ $=40)$, iPSC wt $(n=6)$.

(B) Depicted is the mean tumor size + SEM. ESC OVA $(n=14)$, iPSC OVA $(n=18)$, ESC wt $(n=34)$ iPSC wt $(n=6)$. ESC OVA vs. ESC wt $(p=0.05)$, iPSC OVA vs. iPSC wt $(p=$ $0.79)$, differences between stem cell type: iPSC wt vs. ESC wt $(p=0.46)$, iPSC OVA vs. ESC OVA ( $p=0.36)$; Mann-Whitney U-test (C) Shown is the mean tumor weight $+\operatorname{SEM}$. ESC OVA $(n=13)$, iPSC OVA $(n=18)$, ESC wt $(n=35)$, iPSC wt $(n=5)$. ESC OVA vs. ESC wt $(p=0.05)$, iPSC OVA vs. iPSC wt $(p=0.68)$; differences between stem cell types: iPSC wt vs. ESC wt $(p=0.28)$; iPSC OVA vs. ESC OVA $(p=0.20)$; Mann-Whitney U-test. 
In Table 14, tumor formation rates, tumor size, tumor weight and duration of experiments for each analyzed stem cell clone is depicted. Examination of these parameters for each clone showed that the tumor formation rate was not statistically differing between the various cell clones. In contrast, analysis of tumor size, weight and experimental duration showed significant differences between wildtype cell clones, whereas OVA-expressing cell clones significantly varied in duration, while showing similar tumor size and weight (Table 14, lower rows). This indicates that each clone has its distinct growth rate, leading to varying size and weight of forming tumors. The differing duration of experiments for single clones is promoting this hypothesis, revealing the time differences until tumors reached a certain size and experiments had to be ended. Apart from that, the varying duration of experiments could not be associated with a type of stem cells (ESC, iPSC) or the expression of OVA (data not shown).

Table 14 Analysis of tumor formation, mean tumor size, weight and experimental duration for each stem cell clone after injection into immunodeficient hosts. Significant differences between wildtype or OVA-expressing stem cell clones are depicted in lower rows; Kruskal-Wallis H-test. ${ }^{*}<0.05 ;{ }^{* *}<0.01 ; * * * 0.001$.

\begin{tabular}{|c|c|c|c|c|c|c|}
\hline \multicolumn{7}{|c|}{ Immunodeficient hosts } \\
\hline \multirow{3}{*}{ clone } & \multicolumn{5}{|c|}{ tumor } & \multirow{3}{*}{$\begin{array}{c}\text { duration } \\
\text { [days) }\end{array}$} \\
\hline & \multicolumn{3}{|c|}{ formation } & \multirow{2}{*}{$\begin{array}{c}\text { size } \\
{\left[\mathrm{mm}^{3}\right]}\end{array}$} & \multirow{2}{*}{$\begin{array}{c}\text { weight } \\
\text { [mg] }\end{array}$} & \\
\hline & {$[n$} & of & total] & & & \\
\hline ESC BTL1 & 9 & / & 12 & 272.6 & 0.36 & 40 \\
\hline ESC BTL1 OVA\#4 & 7 & / & 11 & 467.6 & 0.96 & 64 \\
\hline ESC MPI-II & 27 & / & 30 & 858.9 & 1.61 & 50 \\
\hline ESC MPI-II OVA\#4 & 7 & / & 8 & 488.3 & 0.87 & 66 \\
\hline iPSC 129/Sv & 17 & / & 17 & 838.98 & 1.19 & 27 \\
\hline iPSC 129/Sv OVA\#6 & 29 & / & 29 & 267.9 & 0.41 & 26 \\
\hline iPSC 129/Sv OVA\#13 & 4 & / & 4 & 1005.9 & 1.20 & 27 \\
\hline iPSC $129 /$ Sv OVA\#18 & 7 & / & 11 & 412.5 & 1.37 & 47 \\
\hline iPSC 129/Sv OVA\#24 & 8 & / & 9 & 344.88 & 0.46 & 26 \\
\hline $\begin{array}{l}\text { Differences between } \\
\text { OVA clones: } p=\end{array}$ & & & & & & $* * *$ \\
\hline $\begin{array}{l}\text { Differences between } \\
\text { wt clones: } p=\end{array}$ & & & & $*$ & $* *$ & $*$ \\
\hline
\end{tabular}




\subsubsection{Histological analysis of teratomas}

All emerging tumors were histologically analyzed for the development of structures of all three germ layers and if present confirmed as teratomas. In Figure 25, examples are depicted of mesodermal, ectodermal and endodermal structures, which formed after injections of stem cells. All clones that were able to form tumors at high frequency were able to form teratomas and can be considered therefore as pluripotent. Additionally, qPCR analysis was performed to determine the expression of various germ layer marker in the teratomas (Supplement, Ch. 7.2.1).
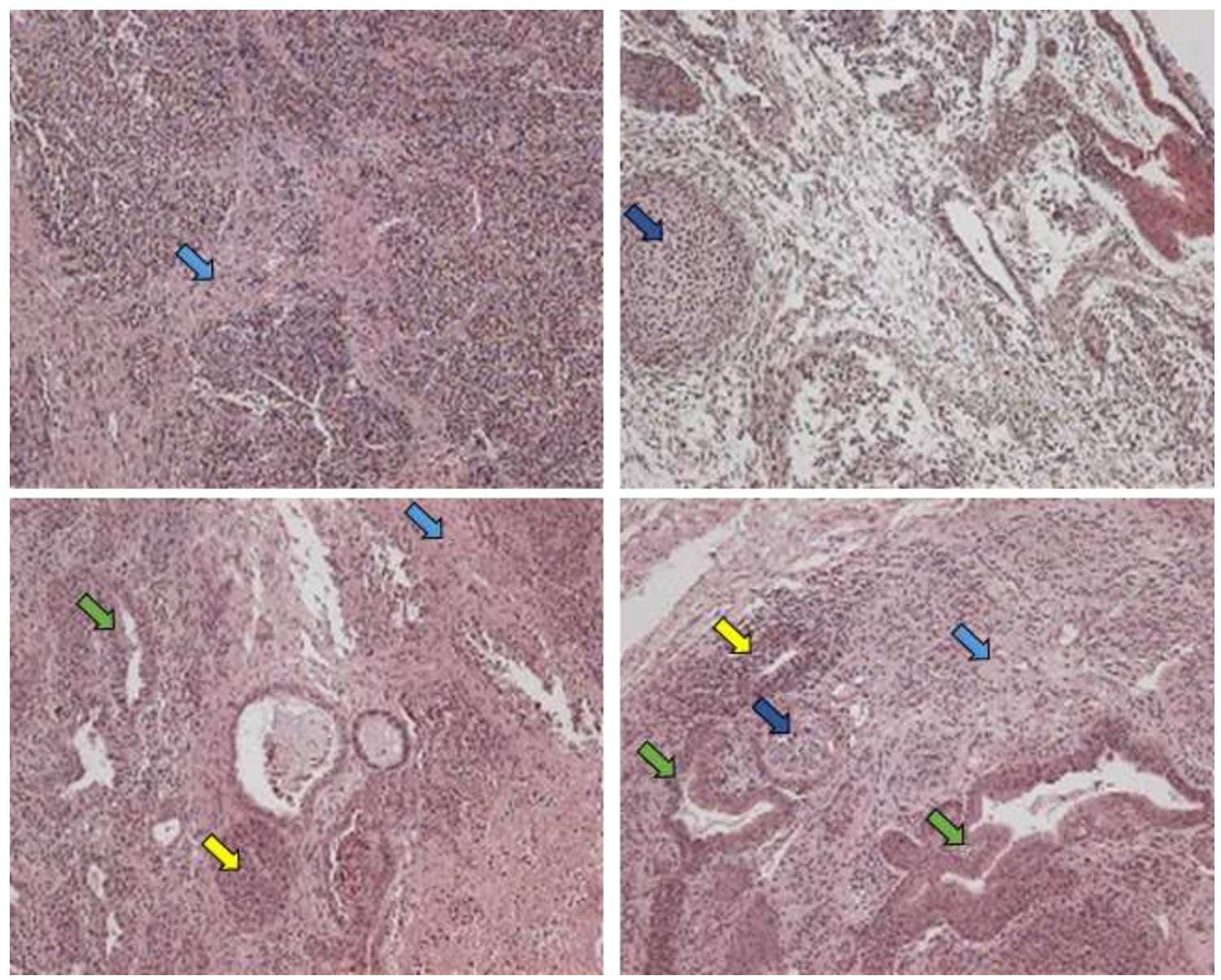

Figure 25 Histological example pictures of teratomas formed after injection of iPSC 129/Sv TD11.1 into immunodeficient RAG2 ${ }^{-/-} \gamma \mathbf{c}^{-/-}$mice.

Teratomas were fixed with formalin and embedded with paraffin for cutting, sections were stained with hematoxylin and eosin (H\&E). The tumor contains derivatives of all germ layers, indicated by colored arrows: yellow: neural-like tissue (ectoderm); light blue: rivulet of muscles/ myocytes (mesoderm); dark blue: cartilage (mesoderm); green: gut epithelium (endoderm). Shown are pictures with 10 fold magnification. 


\subsubsection{Adoptive transfer of OVA-specific T cells into immunodeficient hosts}

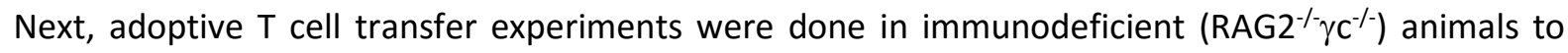
examine whether PSCs are immunosuppressive in vivo as they are in vitro. For these experiments a wildtype iPSC line (iPSC 129/Sv) and two OVA-expressing iPSC clones (iPSC 129/Sv OVA\#6, OVA\#24) were used. It was analyzed whether OVA-specific $\mathrm{CD} 4^{+}$and/or $\mathrm{CD} 8^{+} \mathrm{T}$ cells become activated after transfer and have an effect on tumor formation of injected stem cells. In Figure 26, a schematic overview over the adoptive transfer experiment is depicted.

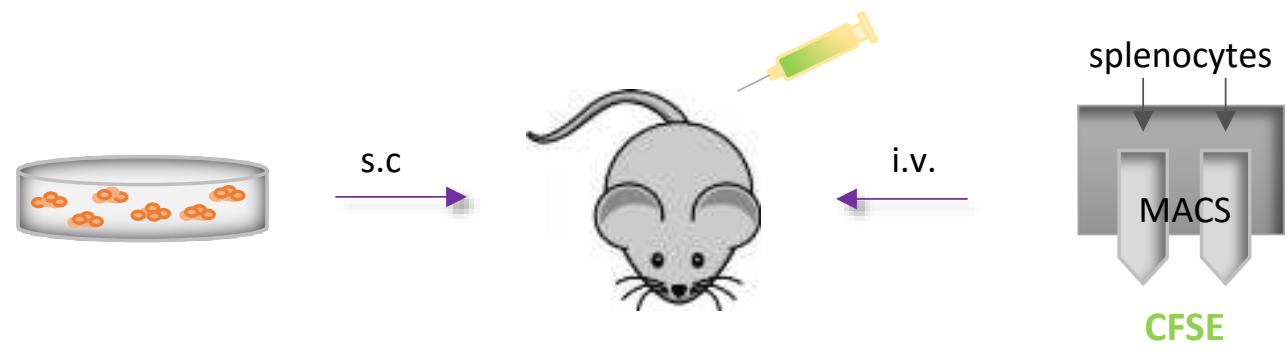

Figure 26 Schematic overview over adoptive transfer experiments performed in immunodeficient RAG2 $^{-1-\gamma c^{-/-} \text {mice. }}$

Wildtype or OVA-expressing stem cells were subcutaneously injected followed by an adoptive transfer (day 4) of transgenic OT-II CD4 ${ }^{+}$cells, OT-I CD8 ${ }^{+}$cells or a mixture of both into the tail vein of the animals $\left(5 \times 10^{6}\right.$ /animal). T cells were labeled with CFSE prior to the transfer to monitor cells in vivo.

OVA-specific T cells were isolated from spleen and lymph nodes of OT-I and OT-II mice via MACS technology and quantified by flow cytometry for expression of the OVA-specific TCR (data not shown). Furthermore, the capacity of T cells to become activated after in vitro stimulation with the respective OVA peptide was monitored. Over $80 \%$ of isolated T cells expressed the transgenic TCRs specific for OVA (TCR V $35.1 / 5.2^{+}$) and showed proliferation after in vitro activation and were therefore considered as suitable for adoptive transfer experiments (data not shown).

Freshly isolated T cells were stained with CFSE and intravenously injected in varying combinations (OT-I T cells, OT-II T cells or a mixture of OT-I/OT-II T cells; $5 \times 10^{6}$ cells /animal) into tail veins of $\mathrm{RAG}^{-/-} \gamma \mathrm{C}^{-/-}$mice four days after injections with wildtype and OVA-expressing stem cells $\left(1.5 \times 10^{6}\right.$ cells /animal). Blood was taken on day 5 after adoptive transfer to monitor whether CFSE-stained T cells could be detected via flow cytometry. Due to missing native T cells in the immunodeficient hosts, all T cells found in this experiment derived from the previously transferred cells. In Figure 27A, it is shown that after transfer of OT-I T cells, up to $23.2 \%$ of total PBMCs were cytotoxic T cells in the 
immunodeficient hosts, which also had received stem cells. In contrast, in mice that had received OT-II T cells, T helper cells constituted 0.5 to $2.8 \%$ of total PBMCs. Analysis of the CFSE staining indicated that cytotoxic $\mathrm{T}$ cells $\left(\mathrm{CD} 3^{+} \mathrm{CD} 8^{+}\right)$had proliferated since they showed a reduced CFSE staining (Figure 27B). Distinct peaks in flow cytometric histograms indicate the cell divisions. T helper cells $\left(\mathrm{CD}^{+} \mathrm{CD}^{+}\right)$, in contrast, did hardly proliferate after adoptive transfer. Notably, the OVA-specific $\mathrm{CD}^{+} \mathrm{CD}^{+} \mathrm{T}$ cells proliferated in recipients which had received OVA-expressing iPSCs as well as in recipients with a wildtype stem cell line or control mice without iPSCs. This indicates a high antigenindependent proliferative capacity of OT-I T cells in lymphocyte-depleted hosts.

A

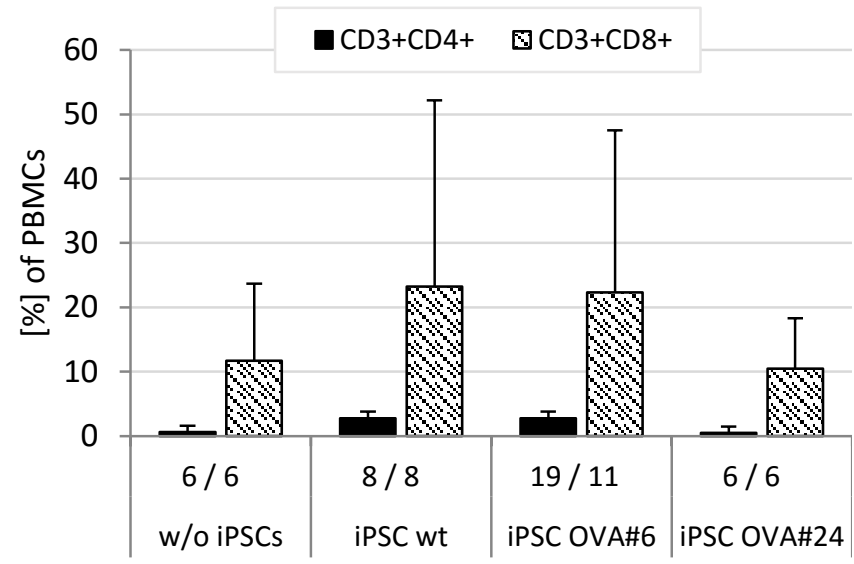

B
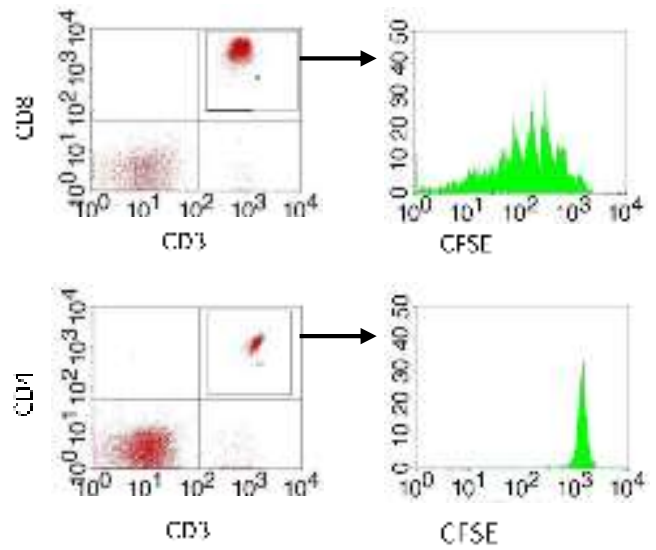

Figure 27 Flow cytometric blood analysis of transferred T cells of RAG2 ${ }^{-1-} \mathrm{cc}^{-/}$mice which previously received different stem cell injections.

Blood samples were taken on day 5 after adoptive transfer of OT-I and OT-II T cells. (A) Shown is the mean proportion + SD of total PBMCs. Animal numbers are indicated directly below the bars. (B) Exemplary measurements of CFSE staining of gated $\mathrm{CD}^{+} \mathrm{CD}^{+}$and $\mathrm{CD} 3^{+} \mathrm{CD} 4^{+}$cell populations. Animals received OVAexpressing stem cells (iPSC OVA\#6) and adoptive transfer of $\mathrm{CD} 8^{+}$or $\mathrm{CD} 4^{+}$TCR-transgenic T cells, respectively. 


\subsubsection{Effect of TCR-transgenic T cells on tumor formation}

To analyze which influence the adoptive transfer of the TCR-transgenic T cells in various combinations had on the injected wildtype or OVA-expressing stem cells, the formation and characteristics of the tumors in the immunodeficient mice were evaluated. This showed that the adoptive T cell transfer had no influence on the tumor formation rate in those animals $(p=0.36)$, displaying tumor formation in almost every performed experiment (Figure 28). Moreover, the size and weight of formed tumors were not significantly altered after T cell transfer (Supplement, Ch. 7.2.2).

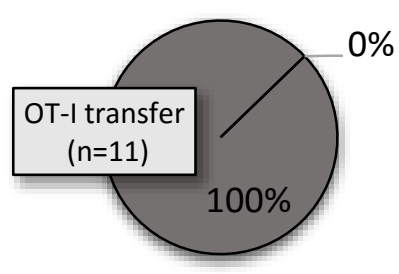

$\square$ tumor $\square$ w/otumor

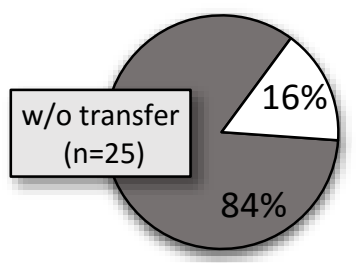

$\square$ tumor $\square$ w/otumor

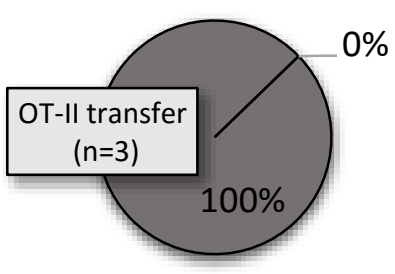

$\square$ tumor $\square$ w/otumor

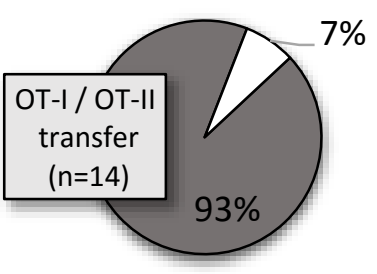

$\square$ tumor $\square$ w/otumor

Figure 28 Tumor formation in immunodeficient hosts, which either additionally received adoptive transfer of OVA-specific T cells (OT-I T cells, OT-II T cells, OT-I/OT-II T cells) or no T cells (w/o transfer).

Numbers of performed experiments are depicted in the respective circle graph.

However, additional histological analysis of tumors showed that teratomas derived from OVAexpressing stem cells were highly infiltrated by $\mathrm{CD}^{+}$cells as depicted in example pictures in Figure 29 . To examine if these T cells were activated, further experiments were performed. 
CD3

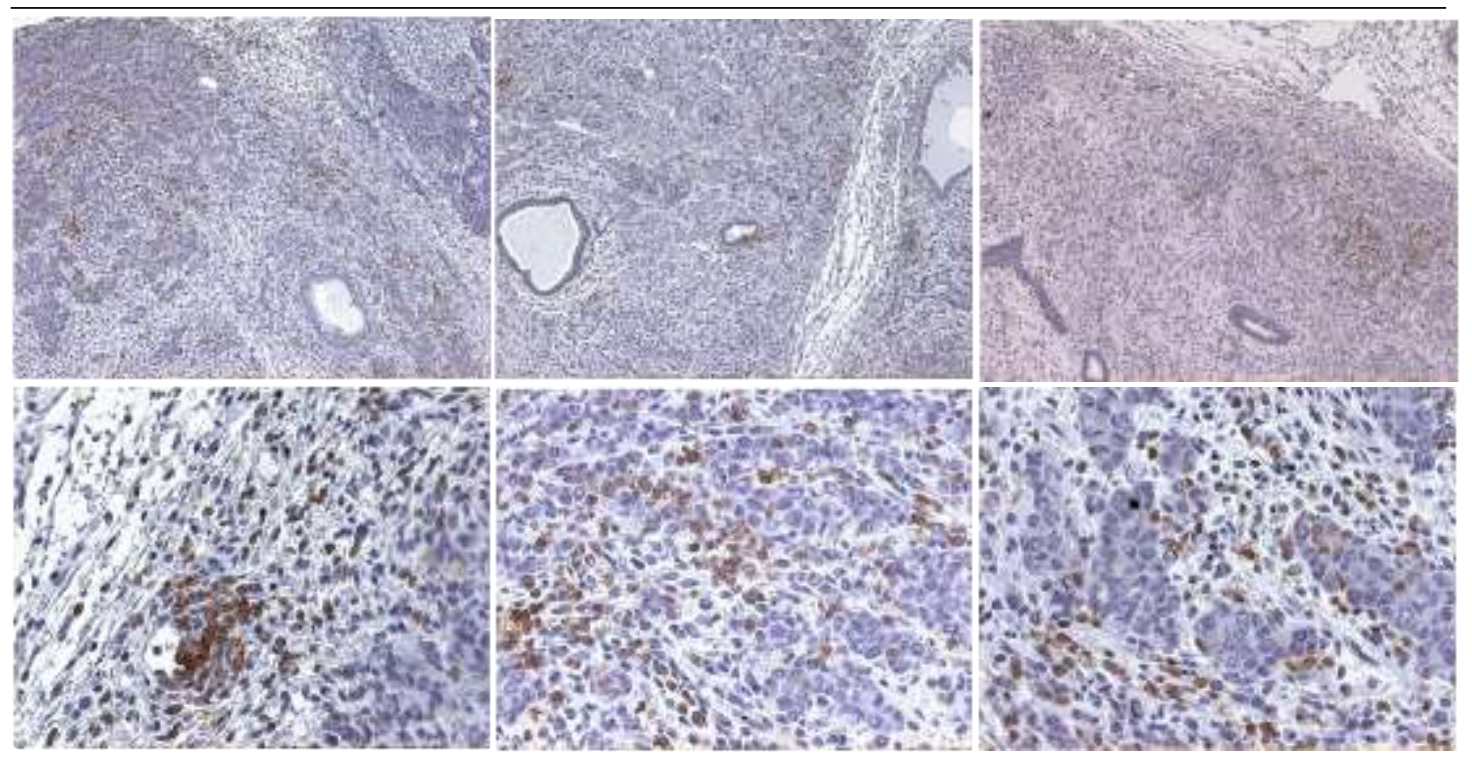

Figure 29 Histological analysis of T cell infiltration into teratomas derived from iPSC 129/Sv OVA\#6 stem cells in RAG2-/- $\gamma \mathrm{c}^{-/-}$mice which additionally received OVA-specific OT-I T cells.

Shown is an immunohistochemical staining of $\mathrm{CD}^{+}$cells in three different teratomas in $100 \mathrm{x}$ magnification (upper row) and $400 \times$ magnification (lower row).

After experimental ending, blood and spleen were collected for further analysis of T cell activation. Even after 40 days post transfer, CFSE-stained cells were still found in blood and spleen of the hosts (around 2-4 \%; data not shown). However, most $T$ cells had lost CFSE staining likely due to proliferation. Splenocytes were directly used in ${ }^{51} \mathrm{Cr}$ release assays to determine the cytotoxic activity of CTLs, shown in Figure 30A for animals which received iPSC 129/Sv OVA\#6 stem cells and adoptive transfer of OT-I T cells. For this, OVA-expressing RMA (eGFP-OVA) and control RMA (eGFP) cells were used as target cell lines. At this, RMA OVA target cells were killed by all splenocytes of animals that previously had received OVA-expressing stem cells and OT-I or OT-I/OT-II T cells (data not shown), showing that OVA-specific CTLs became activated in those animals. In mice carrying teratomas derived from wildtype iPSCs, the CTLs were much less active against the OVA-expressing target cells, indicating the activation of CTLs against an antigen expressed in the iPSCs (Figure 30B). 

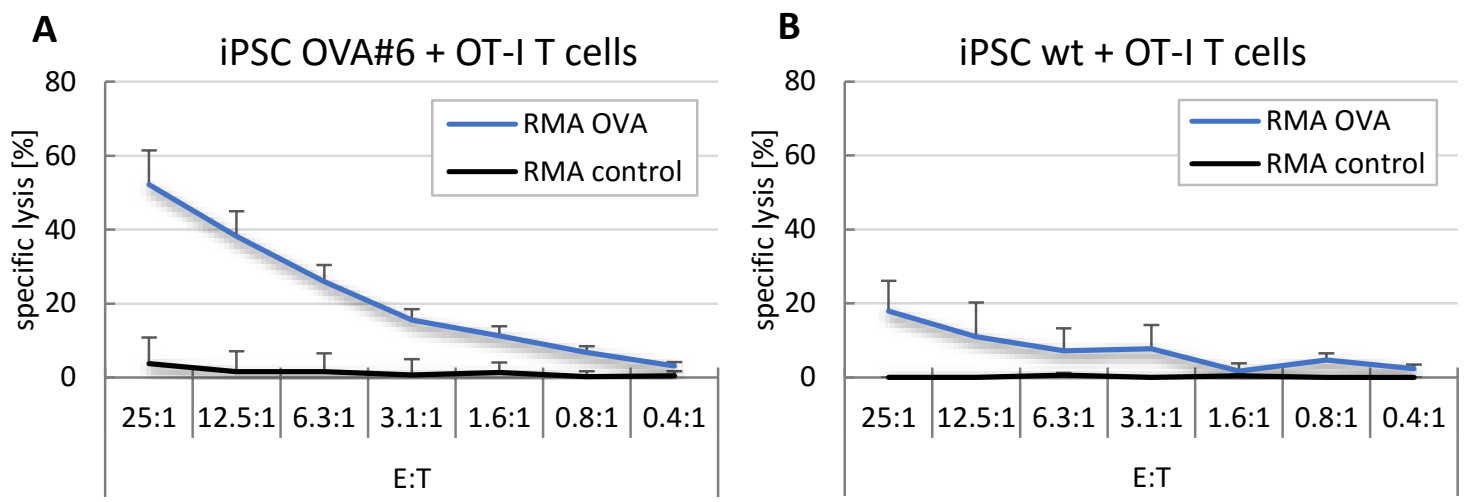

Figure 30 Analysis of OVA-specific CTL activation examined via ${ }^{51} \mathrm{Cr}$ release assays.

Shown is the mean specific lysis of the target cells RMA (eGFP) as control and RMA OVA (eGFP-OVA) by splenocytes of animals which received (A) OVA-expressing or (B) wt 129/Sv iPSCs and OT-I T cells + SEM. OVA $(n=7)$, wt $(n=3)$

Further analysis of survival of animals showed that animals, which received OT-I T cells and displayed high proliferating $\mathrm{CD} 3^{+} \mathrm{CD} 8^{+} \mathrm{T}$ cell in blood analysis (day 5 post transfer) showed a longer survival (Figure 31C). This indicates that the OVA-specific CTLs could inhibit tumor growth of injected stem cells to a certain extent and thereby led to a longer survival in some animals due to delayed tumor growth. Nevertheless, the injected OVA-specific T cells were not sufficient to prevent the growth of teratomas from the stem cells, which were already injected 5 days prior to adoptive transfer of T cells.

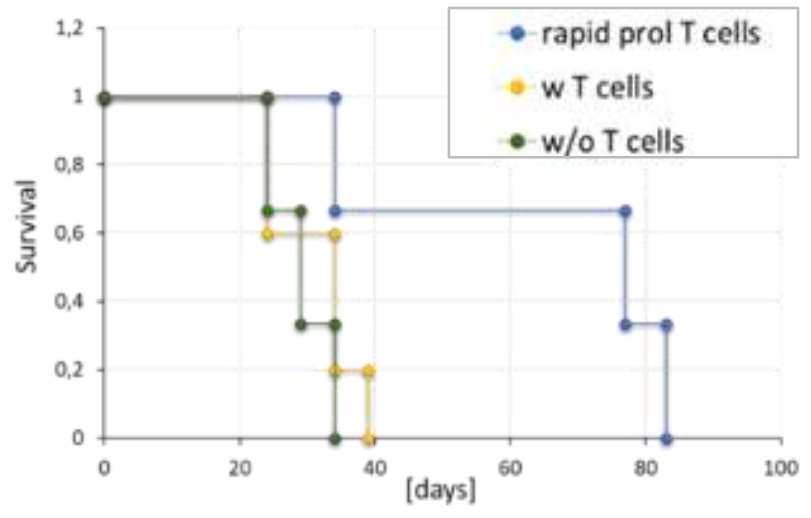

Figure 31 Kaplan-Meier survival curve of immunodeficient animals after inoculation of iPSC 129/Sv OVA\#6 and adoptive transfer of OT-I T cells.

T cells showed various patterns of activation, defined at day 5 post OT-I T cell transfer: rapid proliferating T cells: $\mathrm{CD3}^{+} \mathrm{CD}^{+}: 20-30 \%$ cell count in blood analysis $(n=3)$, w T cells: all other animals which received T cell injections ( $\mathrm{n}$ $=5)$; w/o T cells: received no T cells $(n=3)$. 


\subsubsection{Effect of OVA expression in stem cells on tumor formation in immunocompetent hosts}

Next, stem cells were injected subcutaneously into immunocompetent mice (129/Sv) to monitor whether immune reactions against OVA developed, which represents a single miHC antigen in otherwise syngeneic hosts. Only clones that regularly had formed tumors in the immunodeficient hosts were included in this analysis. After stem cell injections, tumor formation was monitored and differences in the tumor frequency of OVA-expressing and wildtype stem cells were detected (Figure 32). Injections of wildtype stem cells into immunocompetent mice led to a tumor growth in 83 or $85 \%$ of the recipients for ESCs and iPSCs, respectively, which is similar to the tumor rates observed in immunodeficient hosts (Figure 23). In contrast, OVA-expressing stem cells failed to engraft and to form tumors in $62 \%$ of the recipients for iPSC OVA clones and $73 \%$ for ESC OVA clones (Figure 32 ).

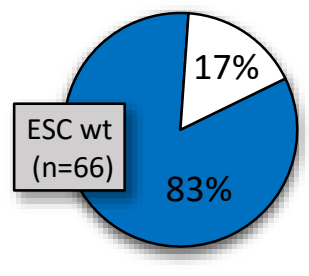

$\square$ w tumor $\square$ w/otumor

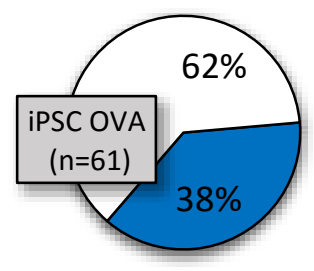

$\square$ w tumor $\square$ w/otumor

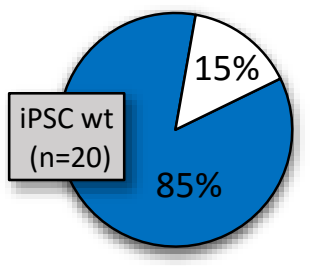

$\square$ w tumor $\square$ w/otumor

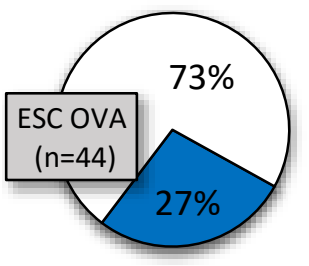

$\square$ w tumor $\square$ w/otumor

Figure 32 Tumor formation of wildtype and OVA-expressing stem cells in immunocompetent 129 /Sv mice.

Numbers of transplanted mice per cell line are depicted in the respective diagrams.

In contrast to wildtype iPSCs and its OVA-expressing clones which derived from one iPS cell line (129/Sv TD11.1), tested ESC clones consisted of different stem cell lines. Besides the previously widely used ESC line MPI-II, the newly generated ESC BTL1 and their OVA-expressing clones were investigated. Analysis of tumor formation of the wildtype ES cell lines and their OVA-expressing clones showed that tumor formation rate of wildtype ESCs were similar, ranging from $81 \%$ (MPI-II) or $86 \%$ (BTL1). However, only in $15 \%$ or $47 \%$ of injections of OVA-expressing ESC BTL1 and MPI-II respectively, 
stem cells were able to engraft, showing variations between the various OVA-expressing cell clones (Figure 33).

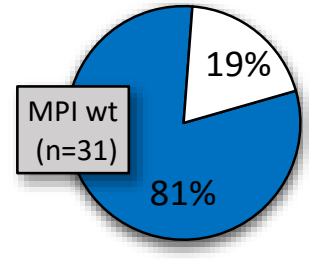

$\square$ w tumor $\square$ w/o tumor

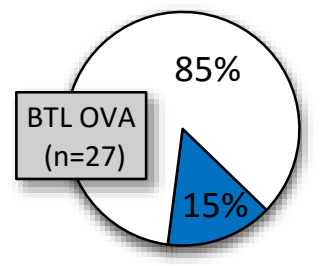

$\square$ w tumor $\square$ w/otumor

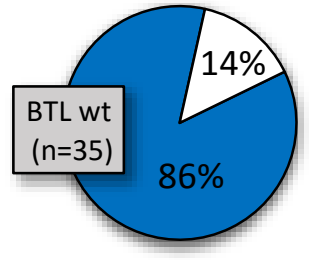

$\square$ w tumor $\square$ w/otumor

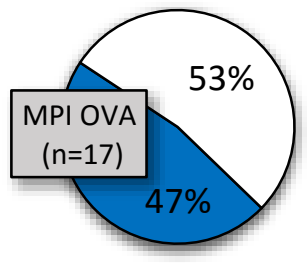

$\square$ w tumor $\square$ w/o tumor

Figure 33 Tumor frequency after ESC injections into immunocompetent 129/Sv host summarized for groups of wildtype ESC BTL1, ESC MPI-II and their derived OVA-expressing clones.

Numbers of transplanted mice per cell line are depicted in the respective diagrams.

\subsection{Effect of OVA expression in stem cells on various parameters in immunocompetent hosts}

Tumors derived from OVA-expressing and wildtype ESCs or iPSCs in immunocompetent 129/Sv mice were confirmed to be teratomas by histology (data not shown) and qPCR analysis (Supplement, Ch. 7.2.1). They were also analyzed for differences in tumor formation rate, size and weight and the influence of recipient gender, stem cell type or expression of OVA. Additionally, the duration until experiments were terminated, due to animal condition and tumor size, were considered for analysis. In contrast to tumor formation in immunodeficient mice, the expression of OVA led to a significantly reduced tumor formation rate in immunocompetent hosts (ESC wt vs. ESC OVA: $p=3.6 \times 10^{-9}$; iPSC wt vs. iPSC OVA: $p=2.4 \times 10^{-4}$; PSC wt vs. PSC OVA: $p=2.9 \times 10^{-12}$; Mann-Whitney U-test; Figure 34A). However, no differences in engraftment of iPSC- or ESC-derived tumors were observed when comparing either wildtype or OVA-expressing stem cell tumors (ESC wt vs. iPSC wt: $p=0.86$; ESC OVA vs. iPSC OVA: $p=0.26$; Mann-Whitney U-test; Figure 34A). Further analysis showed that size and weight significantly varied between tumors derived from OVA-expressing or wildtype stem cells and additionally between wildtype tumors derived from iPSCs and ESCs. In Figure 34B/C, it is displayed that tumors derived from OVA-expressing iPSCs were significantly smaller and lighter compared to 
tumors derived from their wildtype counterparts. Tumors derived from OVA-expressing ESCs were also slightly smaller although not lighter than wildtype tumors. These results suggest that the expression of OVA as a single antigen is sufficient to prohibit engraftment or to lead to a reduction of the size of the stem cell-derived tumors. Nevertheless, significant variation in tumor size and weight were monitored between tumors derived by wildtype iPSCs and ESCS (ESC wt vs. iPSC wt: size ( $p=2.9$ $\left.\times 10^{-4}\right)$, weight $\left(p=8.7 \times 10^{-5}\right)$; Mann-Whitney U-test). Tumors originated from OVA-expressing stem cell displayed variations concerning tumor size (ESC OVA vs. iPSC OVA, $p=0.03$; Mann-Whitney Utest). This might indicate that immune reactions against other differentially expressed antigens between iPSCS and ESCs influence tumor growth. However, these seem to have no major effects on the overall engraftment frequency since only sizee of tumors was affected. Consequently, it is more likely that these differences rather originated from clonal variations between the analyzed cell clones.
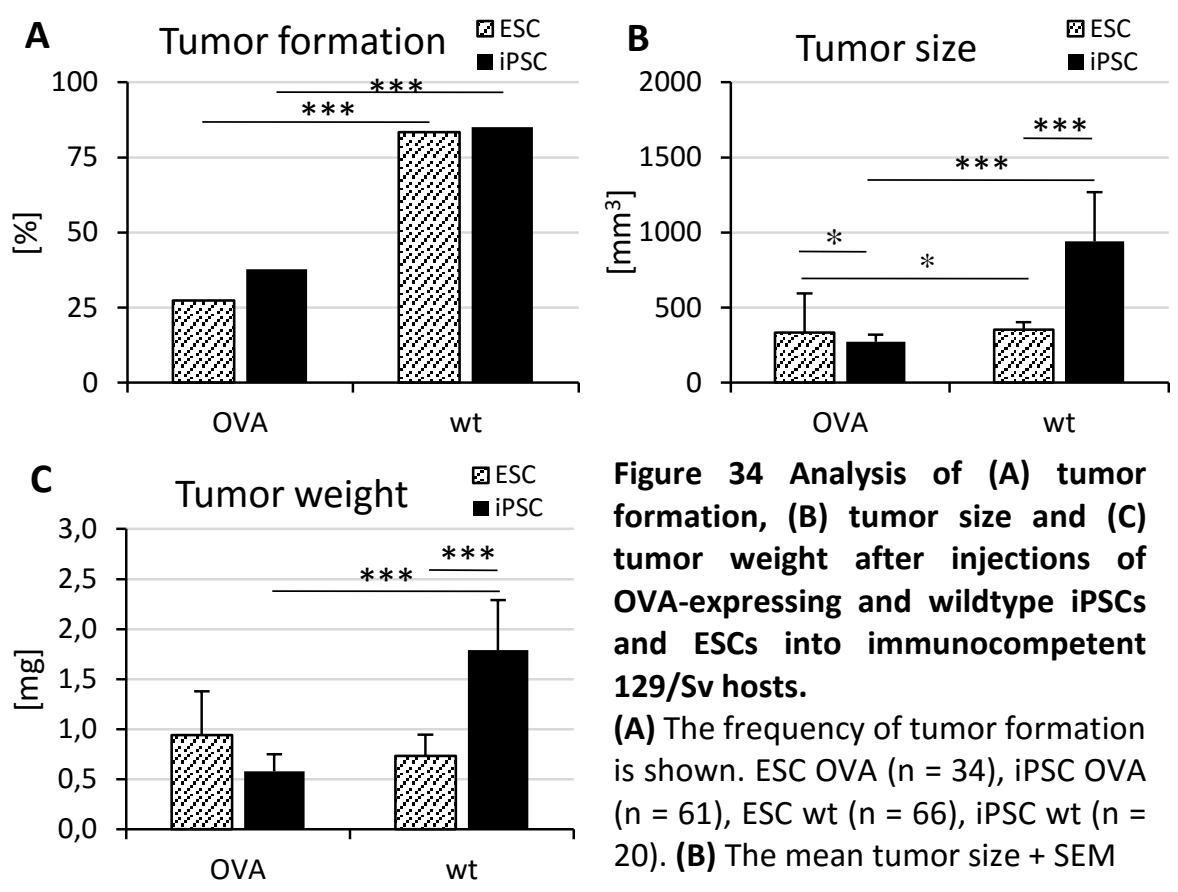

Figure 34 Analysis of (A) tumor formation, (B) tumor size and (C) tumor weight after injections of OVA-expressing and wildtype iPSCs and ESCs into immunocompetent $129 /$ Sv hosts.

(A) The frequency of tumor formation is shown. ESC OVA $(n=34)$, iPSC OVA $(n=61)$, ESC wt $(n=66)$, iPSC wt $(n=$ 20). (B) The mean tumor size + SEM

is depicted. ESC OVA $(n=9)$, iPSC OVA $(n=22)$, ESC wt $(n=53)$, iPSC wt $(n=15)$. ESC OVA vs. ESC wt $(p=0.03)$, iPSC OVA vs. iPSC wt $\left(p=6.5 \times 10^{-5}\right)$, Mann-Whitney U-test. (C) The mean tumor weight + SEM is shown. ESC OVA $(n=12)$, iPSC OVA $(n=23)$, ESC wt $(n=51)$, iPSC wt $(n=17)$. ESC OVA vs. ESC wt $(p=0.23)$, iPSC OVA vs. iPSC wt $\left(p=3.6 \times 10^{-5}\right)$, ESC OVA vs. iPSC OVA $(p=0.48)$, Mann-Whitney Utest. 
The analyzed parameters for single stem cell clones are shown in Table 15, revealing variations in tumor formation frequency for OVA clones (ESC BTL1 OVA\#4; ESC MPI-II OVA\#4; iPSC 129/Sv OVA\#6, \#13, \#18, \#24), but not for wildtype stem cells (ESC BTL1, ESC MPI-II, iPSC 129/Sv) (OVA: $p=3.2 x$ $10^{-3}$; wt: $p=0.84$; Chi-squared test). However, for wildtype stem cell lines significant differences concerning tumor size, tumor weight and duration of the experiments were monitored (size: $p=5.8 \times 10^{-5}$; weight: $p=6.5 \times 10^{-5}$, duration: $p=8.1 \times 10^{-3}$; Kruskal-Wallis H-test). Tumors derived from the OVA-expressing clones showed similar phenotypic characteristics, i.e. tumor size and tumor weight, and similar growth rates, indicated by the duration of experiments (size: $p=0.09$, weight: $p=0.46$, duration: $p=0.07$, Kruskal-Wallis H-test).

Table 15 Analysis of tumor formation, tumor size, tumor weight and experimental duration for each stem cell clone after injection into syngeneic immunocompetent $129 / \mathrm{Sv}$ host. Significant differences between wildtype or OVA-expressing clones are depicted in lower rows. ${ }^{* *}<0.01, * * *<0.001$.

\begin{tabular}{|c|c|c|c|c|c|c|}
\hline \multicolumn{7}{|c|}{ Immunocompetent hosts } \\
\hline \multirow{3}{*}{ clone } & \multicolumn{5}{|c|}{ tumor } & \multirow{3}{*}{$\begin{array}{c}\text { duration } \\
\text { [days] }\end{array}$} \\
\hline & \multicolumn{3}{|c|}{ formation } & \multirow{2}{*}{$\begin{array}{c}\text { size } \\
{\left[\mathrm{mm}^{3}\right]}\end{array}$} & \multirow{2}{*}{$\begin{array}{c}\text { weight } \\
{[\mathrm{mg}]}\end{array}$} & \\
\hline & {$[n$} & of & total] & & & \\
\hline ESC BTL1 & 30 & / & 35 & 234.48 & 0.78 & 30 \\
\hline ESC BTL1 OVA\#4 & 4 & / & 27 & 633.0 & 0.65 & 67 \\
\hline ESC MPI-II & 25 & / & 31 & 488.81 & 0.68 & 64 \\
\hline ESC MPI-II OVA\#4 & 8 & / & 17 & 92.1 & 1.08 & 42 \\
\hline iPSC $129 / \mathrm{Sv}$ & 17 & / & 20 & 941.61 & 1.79 & 17 \\
\hline iPSC 129/Sv OVA\#6 & 10 & / & 19 & 280.9 & 0.81 & 51 \\
\hline iPSC 129/SV OVA\#13 & 1 & / & 6 & 879.6 & 1.39 & 63 \\
\hline iPSC 129/Sv OVA\#18 & 1 & / & 15 & 58.6 & 0,09 & 35 \\
\hline iPSC 129/Sv OVA\#24 & 11 & / & 21 & 227.6 & 0,34 & 36 \\
\hline $\begin{array}{l}\text { Differences between } \\
\text { OVA clones }\end{array}$ & & $* *$ & & & & \\
\hline $\begin{array}{l}\text { Differences between } \\
\text { wt clones }\end{array}$ & & & & $* * *$ & $* * *$ & $* *$ \\
\hline
\end{tabular}

Additionally, it was analyzed whether a distinct sex of injected stem cells and hosts led to a reduced tumor formation in 129/Sv mice after stem cell inoculations. Injections of male stem cells bearing a Ychromosome into female hosts could possibly result in immune reactions against $\mathrm{HY}$ antigens besides the model antigen OVA. Therefore, the tumor formation in male and female hosts was examined. The frequency of teratoma engraftment of wildtype stem cells was similar in male and female recipients (Figure 35). In contrast, injections of OVA-expressing stem cells led to a reduced tumor growth in 
female mice $(8 \%)$ compared to male hosts (46\%) (Analysis for single cell clones: Supplement, Ch. 1.1.1). These data suggest, an additional effect of further miHC antigens such as $H Y$ antigens derived from the Y-chromosome of male stem cells on the rejection of OVA-expressing stem cells or their derivates. However, alternatively stronger immune responses in female than male hosts against OVA could explain this result.
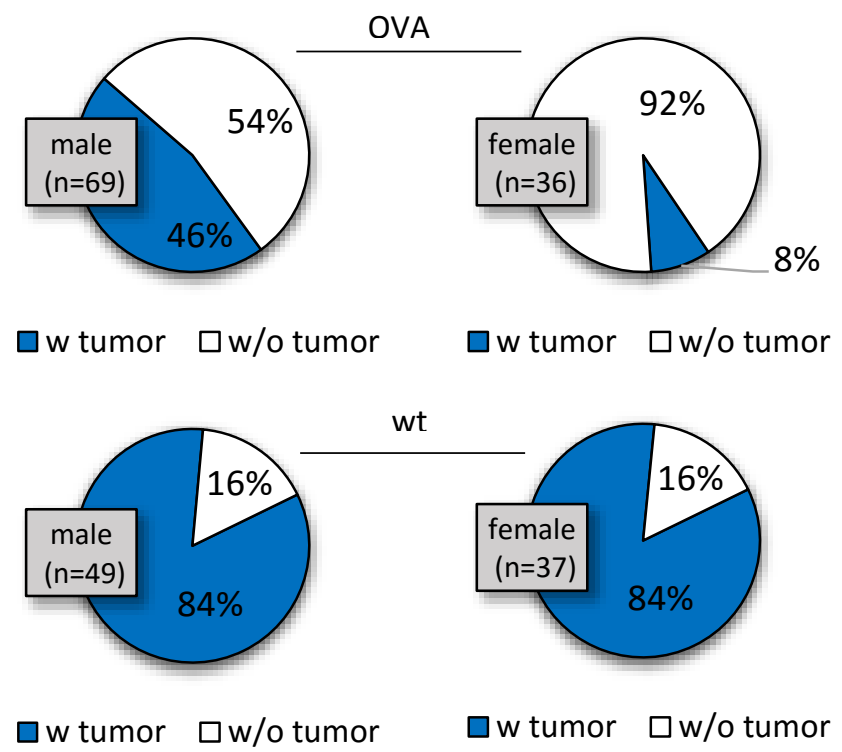

Figure 35 Percentage of tumor formation and rejection in male and female immunocompetent $129 /$ Sv mice after injections of OVA-expressing or wildtype stem cells.

Numbers of performed experiments are depicted in respective graphs.

\subsubsection{Comparative analysis of tumor formation and characteristics in immunodeficient and immunocompetent hosts}

Comparison of tumor formation in immunodeficient (summarized as SCID) and immunocompetent hosts $(129 / \mathrm{Sv})$ revealed that the OVA expression led to a significant reduced engraftment as well as teratoma size and weight in 129/Sv mice compared to immunodeficient hosts (Figure 36), also indicating an effect of OVA expression on the tumor formation. Generally, OVA expression significantly affected tumor frequency and tumor weight only in immunocompetent but not in immunodeficient mice (OVA vs. wt in 129/Sv: formation ( $\left.p=2.9 \times 10^{-12}\right)$, weight $(p=0.01)$; OVA vs. wt in SCID: formation $(p=0.29)$, weight $(p=0.08)$; formation: chi-squared test, weight: Mann-Whitney U-test). The size of wildtype and OVA-expressing tumors was reduced in 129/Sv mice (129/Sv wt vs. 129/Sv OVA: $p=5.6 \times 10^{-3}$; SCID wt vs SCID OVA: $p=0.03$; Mann-Whitney U-test). This observation is in agreement with previous observations that NK cells can delay the growth of teratomas (Dressel et al., 2008, 2010). 
However, the size differences between wildtype and OVA-expressing stem cell-derived tumors in immunodeficient mice (SCID wt vs. 129/Sv wt: $p=0.01$; Mann-Whitney U-test) suggest clonal differences in the in vivo proliferation rate as a further explanation. The tumor weight showed a similar pattern in the different hosts. The duration of experiments before termination due to tumor size was not significantly altered by immune status of the hosts (immunocompetent or immunodeficient) or OVA expression (SCID wt vs SCID OVA: $p=0.72 ; 129 / S v$ wt vs. 129/Sv OVA: $p=0.73 ;$ SCID wt vs. 129/Sv wt: $p=0.52$; SCID OVA vs. 129/Sv OVA: $p=0.68$, Mann-Whitney U-test; data not shown).
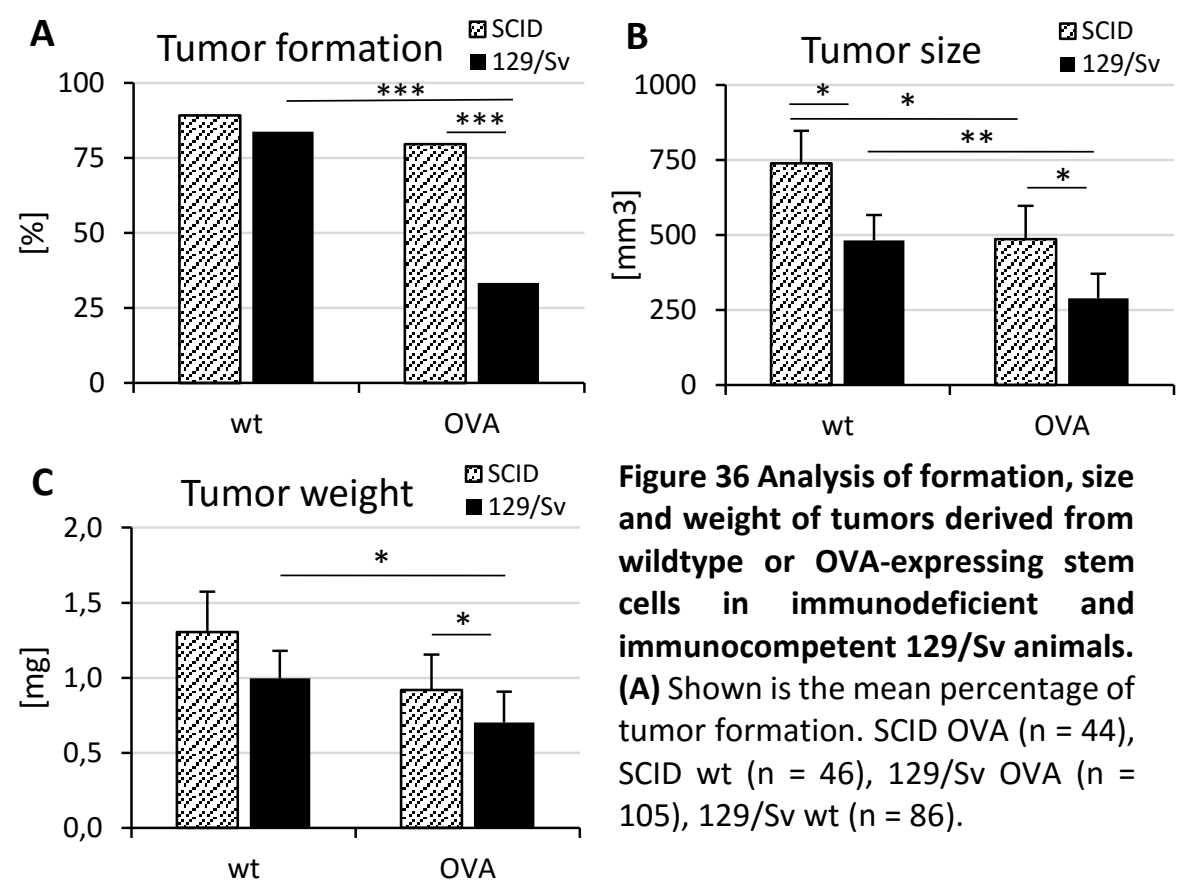

Figure 36 Analysis of formation, size and weight of tumors derived from wildtype or OVA-expressing stem cells in immunodeficient and immunocompetent 129 /Sv animals. (A) Shown is the mean percentage of tumor formation. SCID OVA $(n=44)$, SCID wt $(n=46), 129 /$ Sv OVA ( $n=$ 105), $129 /$ Sv wt $(n=86)$.

OVA SCID vs. OVA $129 / \mathrm{Sv}\left(p=2.5 \times 10^{-7}\right)$, wt SCID vs. wt $129 / \mathrm{Sv}(p=0.39)$, SCID OVA vs. SCID wt $(p=0.29)$, Mann-Whitney U-test. (B) Depicted is the mean tumor size + SEM. SCID OVA $(n=32)$, SCID wt $(n=40), 129 /$ Sv OVA $(n=31)$, $129 /$ Sv wt $(n=68)$. SCID vs. OVA $129 /$ Sv $(p=0.016)$, wt SCID vs. wt $129 /$ Sv $(p=$ $0.01)$, Mann-Whitney U-test (C) Shown is the mean weight of tumors + SEM. SCID OVA ( $n=32)$, SCID wt $(n=40), 129 /$ Sv OVA $(n=31), 129 /$ Sv wt $(n=68)$. OVA SCID vs. OVA $129 / \mathrm{Sv}(p=0.05)$, wt SCID vs. wt $129 / \mathrm{Sv}(p=0.08)$, Mann-Whitney U-test.

\subsubsection{Analysis of teratomas}

Next, teratomas were further analyzed via qPCR for expression of pluripotency and proliferation markers, transgene expression and additional markers, which were previously suggested to be involved in stem cell immunogenicity or immune escape mechanisms, respectively. 


\subsubsection{Characterization of pluripotency of tumor cells}

Expression of marker genes for the three germ layers was found in the tumors (Supplement; Ch. 7.2.4). Thus they were confirmed to be teratoma cells also at the molecular level, it was then analyzed whether teratomas still express markers of pluripotent cells. For this, Nanog and Oct4, two pluripotency markers were examined for their expression levels in teratomas. The relative expression of Nanog and Oct4 in teratomas was calculated compared to the corresponding PSC line, which was set to 1 . As shown in Figure 37, the relative expression of both markers varied in teratomas but generally they showed a lower expression level $(<1)$ in teratomas than pluripotent control cell lines (set as 1). The expression of Nanog was usually higher than Oct4. In summary, after injection of stem cells into hosts, cells start to differentiate into more mature cell types expressing markers of specific germ layers, while losing their pluripotency. However, pluripotent cells appear to be present in teratomas.

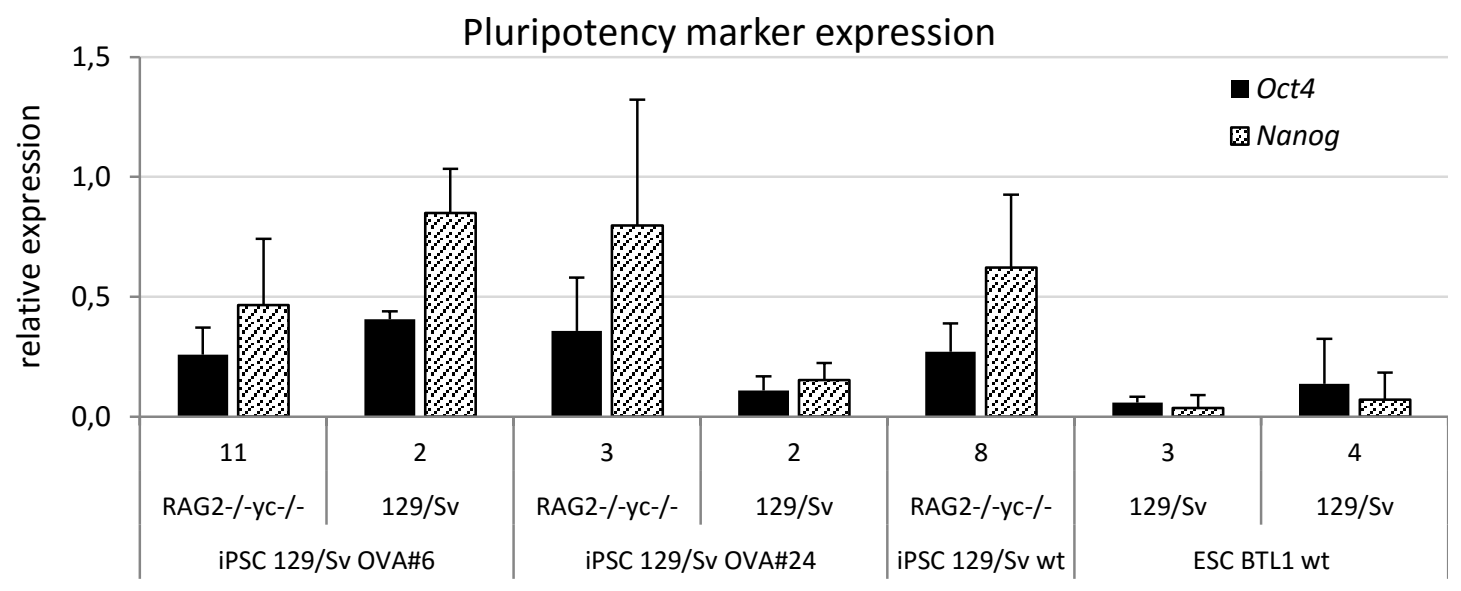

Figure 37 QPCR analysis of pluripotency and transgene markers in teratomas derived from various injected stem cells into immunodeficient (RAG2 $2^{-/ \gamma c /-}$ ) and immunocompetent (129/Sv) syngeneic hosts.

Shown is the relative expression of pluripotency marker Oct4 and Nanog + SD in teratomas formed after injections of wildtype (ESC BTL1, iPSC 129/Sv) and OVA-expressing (iPSC 129/Sv OVA\#6 and \#24) stem cells compared to the PSC lines (set as 1). Numbers of analyzed teratomas are depicted directly below the colums in the graph.

Additionally, teratomas were examined by IHC, revealing frequently areas positive for the pluripotency marker OCT4 and the proliferation marker KI67 as seen Figure 38. Thus even after several weeks following transplantation (up to 90 days), pluripotent stem cells were found within teratoma tissue. 


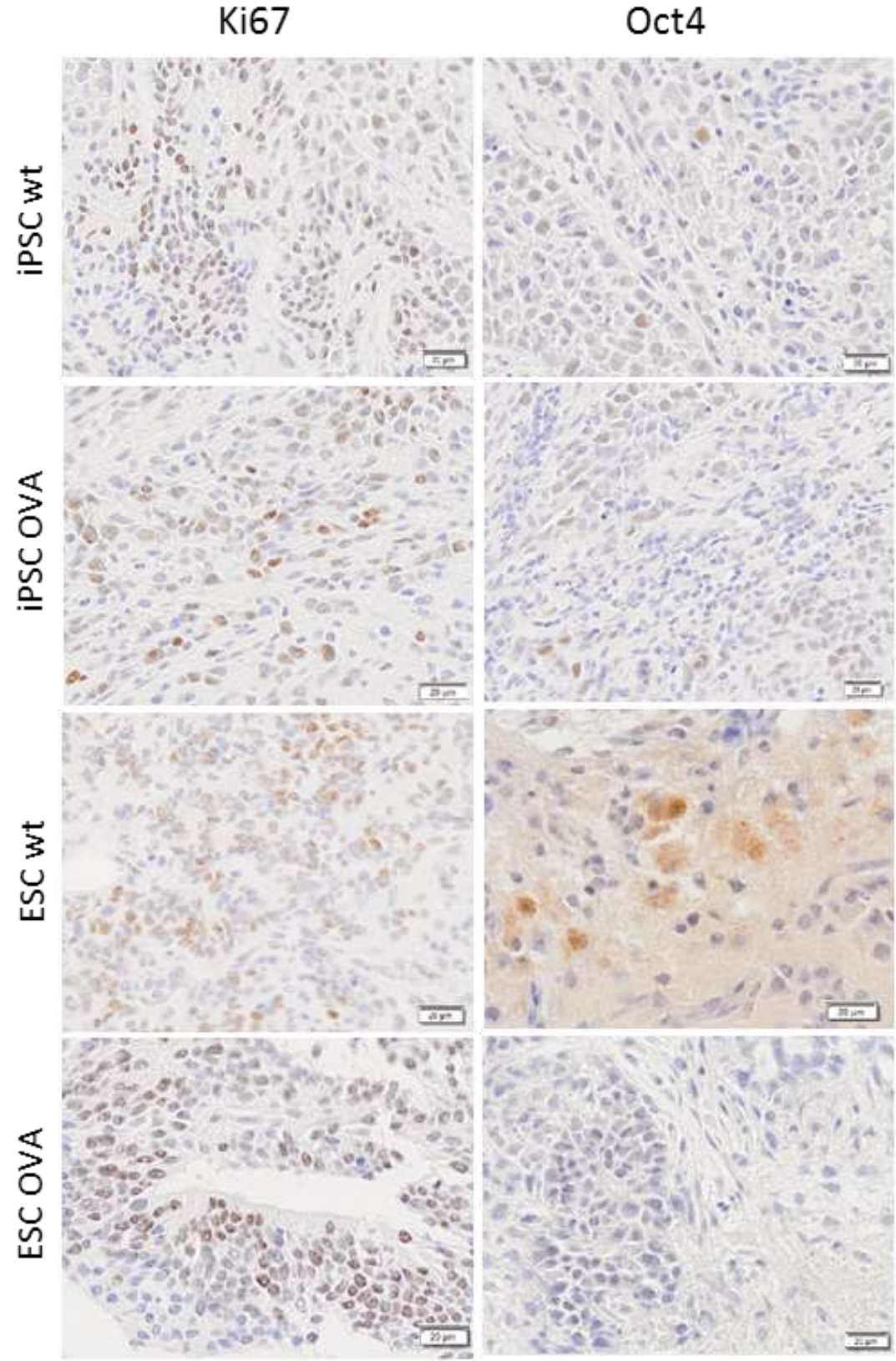

Figure 38 Analysis of cell proliferation and pluripotency via IHC stainings of teratoma tissue derived from wildtype and OVAexpressing stem cell injections into $129 / \mathrm{Sv}$ hosts.

Shown are IHC stainings with antibodies against the pluripotency marker OCT4 and the proliferation marker KI67 for exemplary teratomas. iPSC wt: iPSC 129/Sv, iPSC OVA: iPSC 129/SV OVA\#6, ESC wt: ESC BTL1, ESC OVA: ESC BTL1 OVA\#4. Scale bars in pictures (bottom right corner) indicate $20 \mu \mathrm{m}$ each.

\subsubsection{Transgene expression in teratomas}

Because the effects of the miHC antigen OVA expressed in various stem cells is the basis for this study, it was examined whether the transgene OVA-eGFP was expressed after differentiation in teratomas as in the PSC lines. In Figure 39, it is shown that the relative expression of the transgene was dramatically reduced in many teratomas down to 0.01 fold of the expression in PSCs. Variations occurred between different injected clones, e.g. teratomas derived from iPSC 129/Sv OVA\#24 showed 
a minor reduction ( 0.73 to 0.34 fold) in transgene expression compared to other teratomas ( 0.01 to 0.1 fold). However, the loss of transgene in tumors derived from iPSC OVA\#24 was higher in immunocompetent animals, which indicates that immune cells might have caused this reduction due to elimination of OVA-expressing cells. In contrast, expression in teratomas derived from iPSC OVA\#6 showed similar levels of low transgene expression, leading to the assumption that epigenetic changes while differentiation silenced the transgene locus and consequently expression was lost. In summary, it was shown that transgene expression can get lost after differentiation, which has to be considered in the evaluation of immune reactions against OVA.

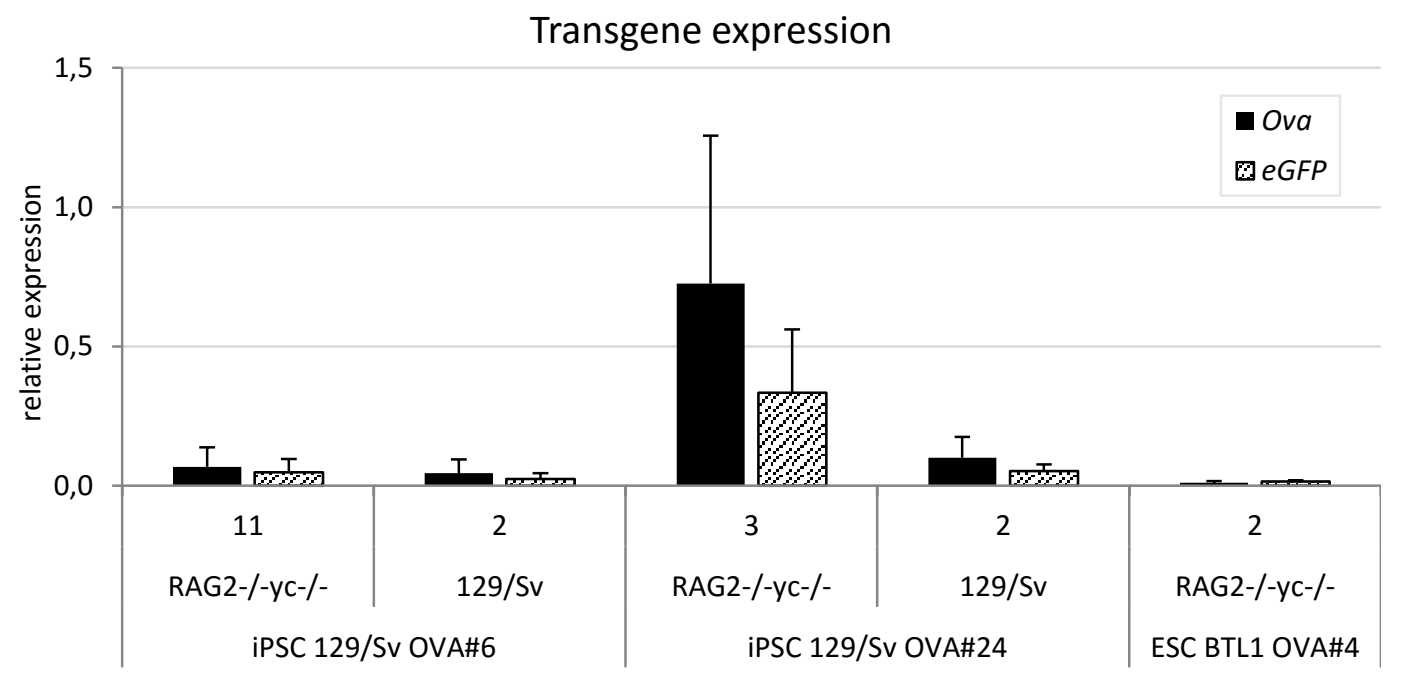

Figure 39 QPCR analysis of transgene markers in teratomas derived from injections of various stem cells into immunodeficient (RAG2 $\left.{ }^{-/} \mathrm{\gamma c}^{-/}\right)$and immunocompetent $(129 / \mathrm{Sv})$ syngeneic hosts. Shown is the relative expression of transgene marker Ova and eGFP + SD in teratomas derived from OVA-expressing stem cell lines (iPSC 129/SV OVA\#6, \#24; ESC BTL1 OVA\#4) compared to pluripotent precursors (set as 1). Numbers of analyzed teratomas are depicted directly below the colums in the graph.

\subsubsection{Expression of amino acid-depleting enzymes in teratomas}

To analyze whether teratomas display an immune escape mechanism that was described for stem cells, i.e. T cell suppression by expression of special amino acid depleting enzymes, the expression of the respective genes was examined. This included expression analysis of Arg1, which leads to depletion of L-arginine if highly expressed and results in T cell suppression and Ido, which is reported to act on T cell activity by degrading tryptophan (Munn and Mellor, 2007; Yachimovich-Cohen et al., 
2010). Figure 40 shows the relative expression of both genes compared to liver tissue as positive control for $\operatorname{Arg} 1$ (set as 1) and to placenta as control for Ido (set as 1). Both genes were expressed in all PSCs on a minimal level compared to positive controls (Ido: $6.8 \times 10^{-8}$ to $5 \times 10^{-3}$ fold, $\operatorname{Arg} 1: 4.6 \times$ $10^{-4}$ to $4 \times 10^{-3}$ fold). Expression increased with differentiation and teratomas showed a constant expression of Arg1 at a low level between 0.02 to 0.08 fold of control, whereas Ido was also low in PSCs but became increased in teratomas ( 0.02 to 0.53 fold). However, in single teratomas derived from wildtype ESC BTL1, levels higher than control levels of placenta were reached (1.9 to 22.7 fold). Thus, the overexpression of Ido could be responsible for a failing immune recognition of teratomas in single cases but not regularly.

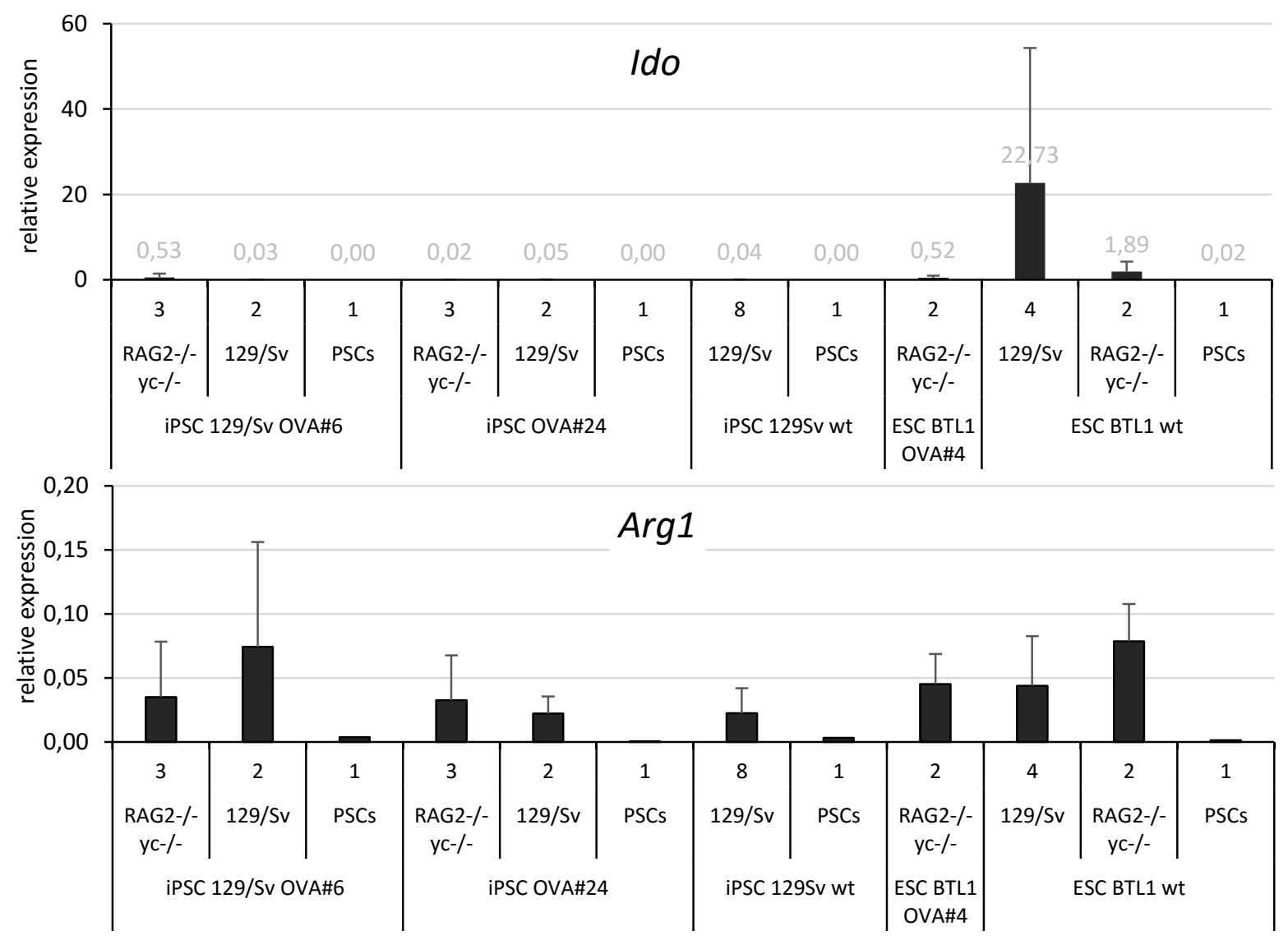

Figure 40 QPCR analysis of $I d o$ and $A r g 1$ gene expression in teratomas derived from various stem cell clones into immunocompetent $(129 / \mathrm{Sv})$ or immunodeficient (RAG2 $2^{-/} \mathrm{\gamma c}^{-/-}$) hosts and their PSC lines.

Shown is the mean relative expression of Arg1 compared to liver tissue (129/Sv) and Ido expression compared to placenta tissue (129/Sv) expression + SD. Numbers of analyzed teratomas are depicted below each column. Hprt was used as housekeeper for Ido expression normalization, for Arg1 the geometric mean of Gapdh and Hprt was used. 


\subsubsection{Expression of immunogenicity-causing genes}

In the literature it was reported that transplanted stem cells show an overexpression of so-called 'immunogenicity-causing' genes namely Cyp3a11, Zg16 and Hormad1 (Zhao et al., 2011). To test whether the PSCs analyzed here as well as the teratomas derived from them express these genes, qPCR analysis was performed. In Figure 41, the relative expression of these genes in wildtype stem cells and teratomas is depicted, showing an overall low expression of each gene. Expression of Cyp3a11 ranged from $6.7 \times 10^{-3}$ to 0.01 in PSCs and from $4.4 \times 10^{-3}$ to 0.07 in teratomas compared to liver tissue. Hormad1 showed even lower expression than Cyp3a11 ranging on minimal levels of $2.1 \times 10^{-3}$ to $8.1 \times 10^{-3}$ in PSCs and from $5.6 \times 10^{-4}$ to $9.2 \times 10^{-3}$ in teratomas compared to testis tissue expression. Relative $\mathrm{Zg} 16$ expression showed the lowest expression of $9.5 \times 10^{-9}$ to $8.1 \times 10^{-3}$ fold for PSCs and $1.5 \times 10^{-6}$ to $1.6 \times 10^{-4}$ in teratomas compared to lung tissue expression. Generally, these genes were only expressed on a minimal level and it appears to be unlikely that these genes have major impacts on the immunogenicity of stem cells and derived teratomas.

In summary, general analysis of teratomas showed that stem cells differentiated into more mature cell types, thereby partly losing their transgene expression. However, residuals of pluripotent cells and proliferating cells were still found in certain areas in the teratomas. On gene expression level, tumors neither showed strong activation of immune escape mechanisms based on depletion of amino acids nor high expression of previously suggested immunogenicity-causing genes. 


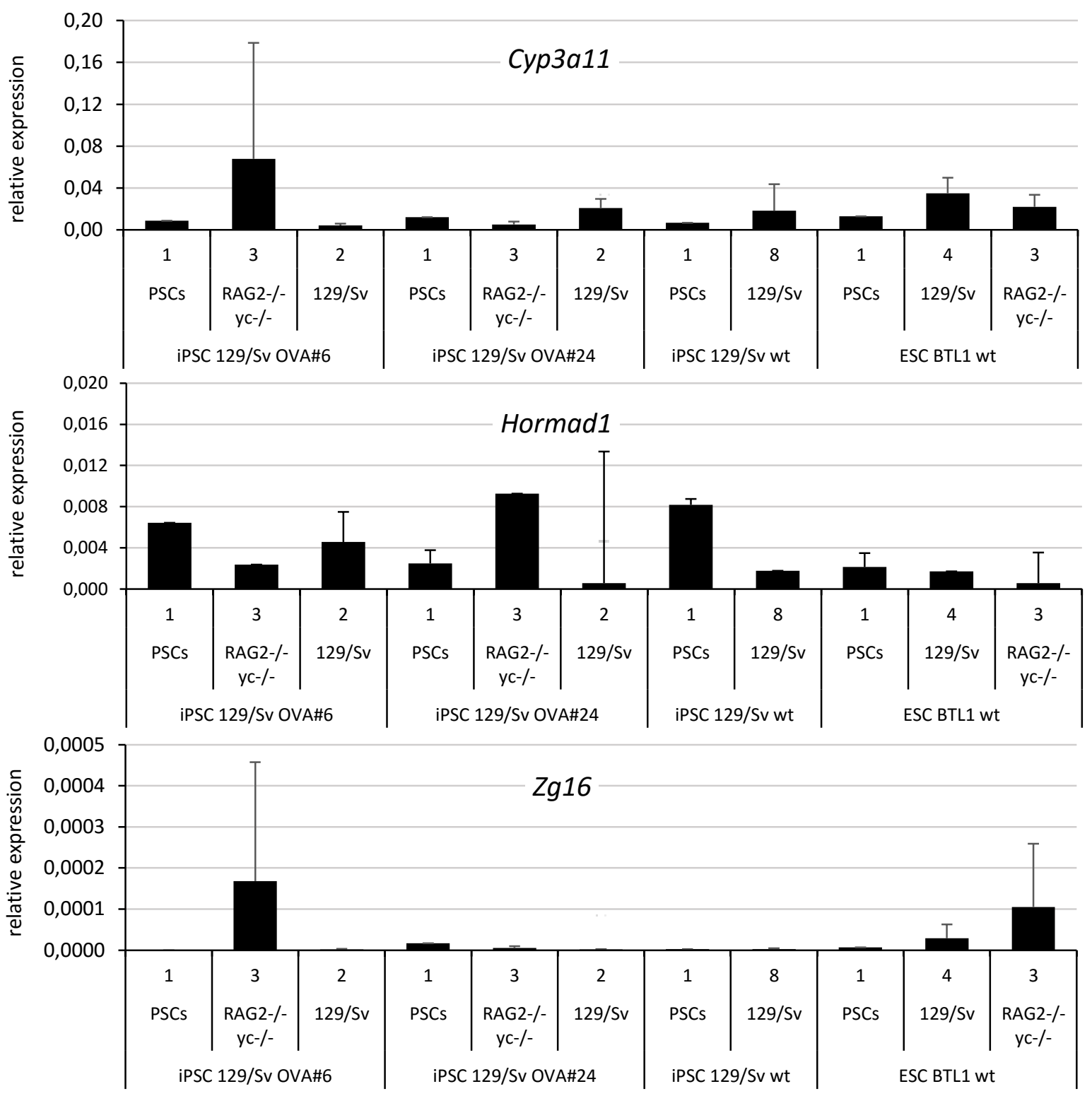

Figure 41 QPCR analysis of 'immunogenicity-causing' gene expression in teratomas derived from injections of various stem cell clones into immunocompetent (129/Sv) or immunodeficient (RAG2 ${ }^{\left.-/-\gamma c^{-/}\right)}$) hosts and their PSC lines.

Shown is the mean relative expression of Cyp3a11 compared to liver tissue (129/Sv), Hormad1 expression compared to testis (129/Sv) and Zg16 expression compared to lung tissue (129/Sv) expression + SD. Numbers of analyzed teratomas are indicated directly below the colums.

\subsubsection{Infiltration into teratomas}

To further characterize the effects that the expression of OVA as miHC antigen might have on the inoculated stem cells, leukocyte infiltration into teratomas derived from OVA-expressing and wildtype stem cell in $129 /$ Sv mice was investigated by IHC. 


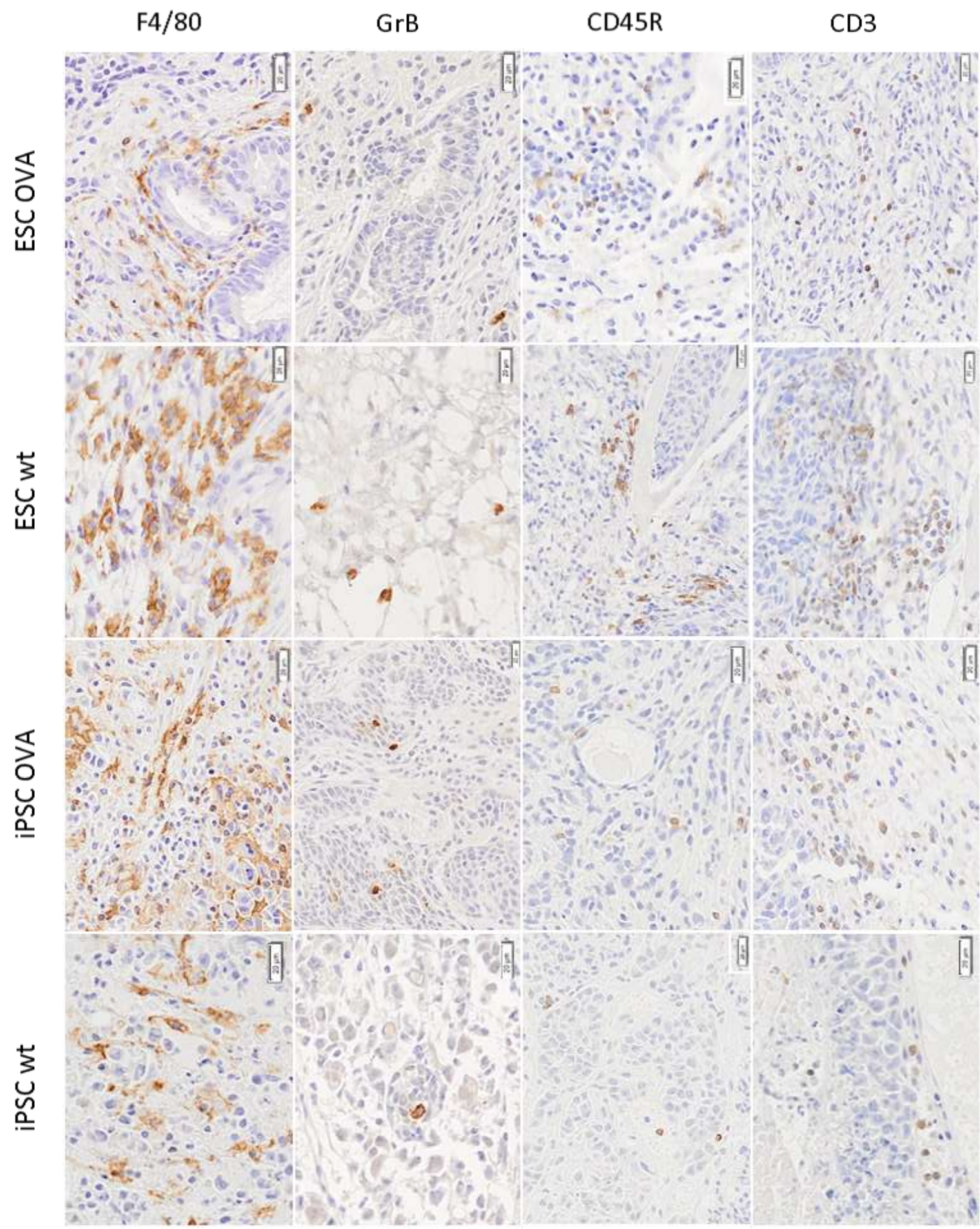

Figure 42 IHC stainings of teratoma tissue derived from wildtype and OVA-expressing stem cell injections into 129 /Sv hosts, analyzed for infiltrating leukocytes.

IPSC wt: iPSC 129/Sv, iPSC OVA: iPSC 129/Sv OVA\#6, ESC wt: ESC BTL1, ESC OVA: ESC BTL1 OVA\#4; Scale bars (upper right corner) indicate $20 \mu \mathrm{m}$ each 
Analysis showed that tumors were infiltrated to a distinct extend by various leukocyte populations including T cells $\left(\mathrm{CD}^{+}\right)$, macrophages $\left(\mathrm{F} 4 / 80^{+}\right)$and $B$ cells $\left(C D 45 R^{+}\right)$(Figure 42$)$. Granzyme $B(G r B)$ was included as a marker of cytotoxic cells such as activated CTLs and NK cells, and single cells positive for this marker were found in the teratomas.

The leukocyte infiltration into teratomas derived from OVA-expressing stem cells and wildtype stem cells were further quantified by a semi-quantitative histological analysis and flow cytometry. IHC analysis showed that $\mathrm{CD}^{+}, \mathrm{CD} 45 \mathrm{R}^{+}, \mathrm{F} 4 / 80^{+}$and $\mathrm{CD} 8^{+}$cells were found in teratomas derived from wildtype and OVA-expressing stem cells (Figure 43). However, if signals were classified in groups of (1) single positive cells, (2) groups of positive cells and (3) confluent groups of positive cells, tumors derived from OVA-expressing stem cells showed higher infiltration of these immune cell populations than tumors derived from wildtype stem cells. Additionally, leukocyte infiltration was examined in single teratomas by flow cytometry, confirming the infiltration of several leukocyte populations by another method (Supplement, Ch. 7.2.4).

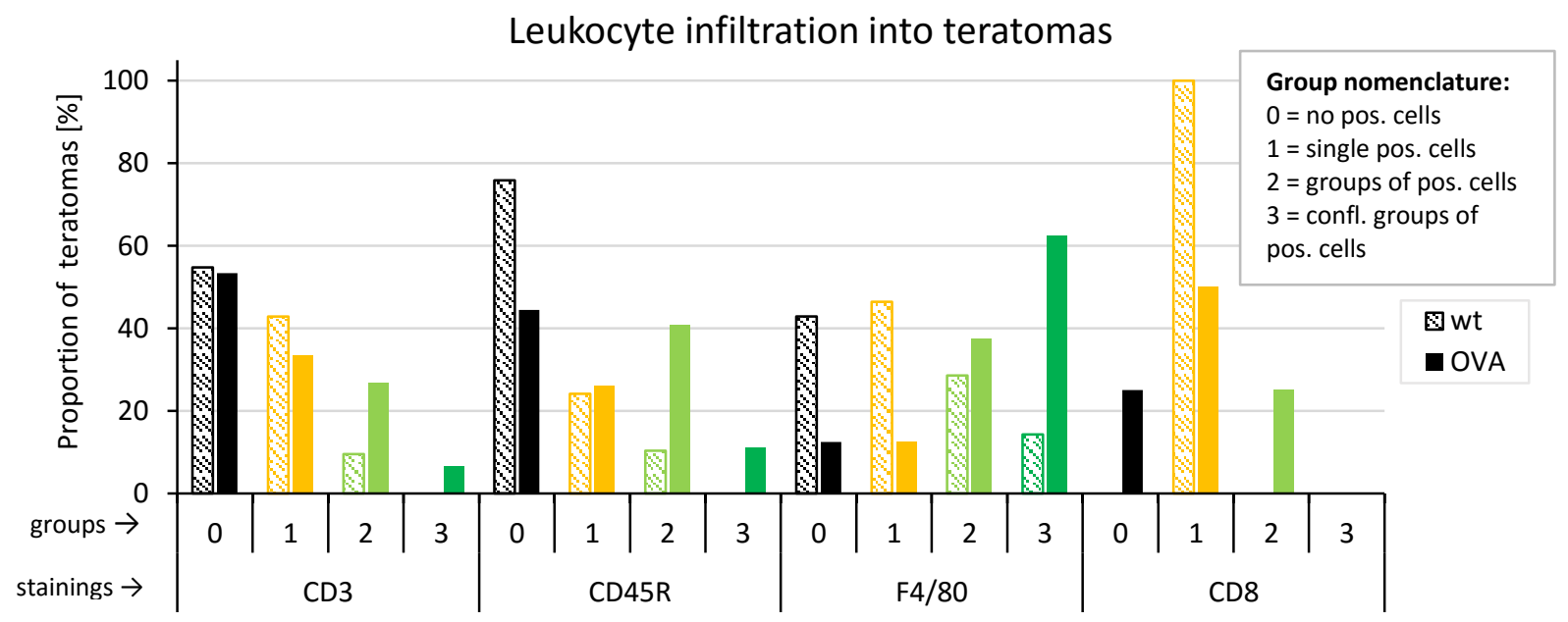

Figure 43 Analysis of leukocyte infiltration into teratomas analyzed by IHC.

Proportion of leukocyte marker-positive cells for classified groups. Histological tissue slides of tumors were stained with antibodies against various leukocyte markers and positive signals were grouped by manual analysis. Visible positive stained cells were classified in either $0=$ no staining, $1=$ single positive cells, $2=$ groups of positive cells or $3=$ confluent groups of positive cells. Teratomas derived from wildtype stem cells: CD3 ( $n=42), \operatorname{CD} 45 R(n=22), F 4 / 80(n=28), C D 8(n=4)$; teratomas derived from OVA-expressing stem cells: CD3 $(n=30), \operatorname{CD} 45 R(n=12), F 4 / 80(n=8), \operatorname{CD} 8(n=4)$. 


\subsubsection{Generation of OVA-specific CTLs in syngeneic hosts}

Next, it was clarified whether OVA-specific T cells arose in animals which received OVA-expressing stem cells in contrast to animals which received wildtype stem cells. Cytotoxicity tests were performed using splenocytes obtained from 129/Sv hosts which previously received injections of wildtype and OVA-expressing stem cells. The experiments were performed at the experimental end points, after 90 days or when animals had to be sacrificed due to tumor growth (long term experiments), or after 1 week (short-term experiments). The splenocytes were restimulated in vitro for 4 days with OVA protein before the ${ }^{51} \mathrm{Cr}$ release assays. Analysis of splenocyte composition, including $\mathrm{T}$ helper cells, cytotoxic T cells, NK cells and specific TCR V 3 5.1/5.2 T cells, showed no major differences between animals which received wildtype or OVA-expressing stem cells or which showed tumor formation or tumor rejection (Figure 44; Supplement, Ch. 7.2.5, unstimulated). The E:T ratio for effector cells in the cytotoxicity assays was adjusted to the proportion of $\mathrm{CD}^{+} \mathrm{CD}^{+}$cells of the restimulated splenocyte population detected via flow cytometry on day 4.

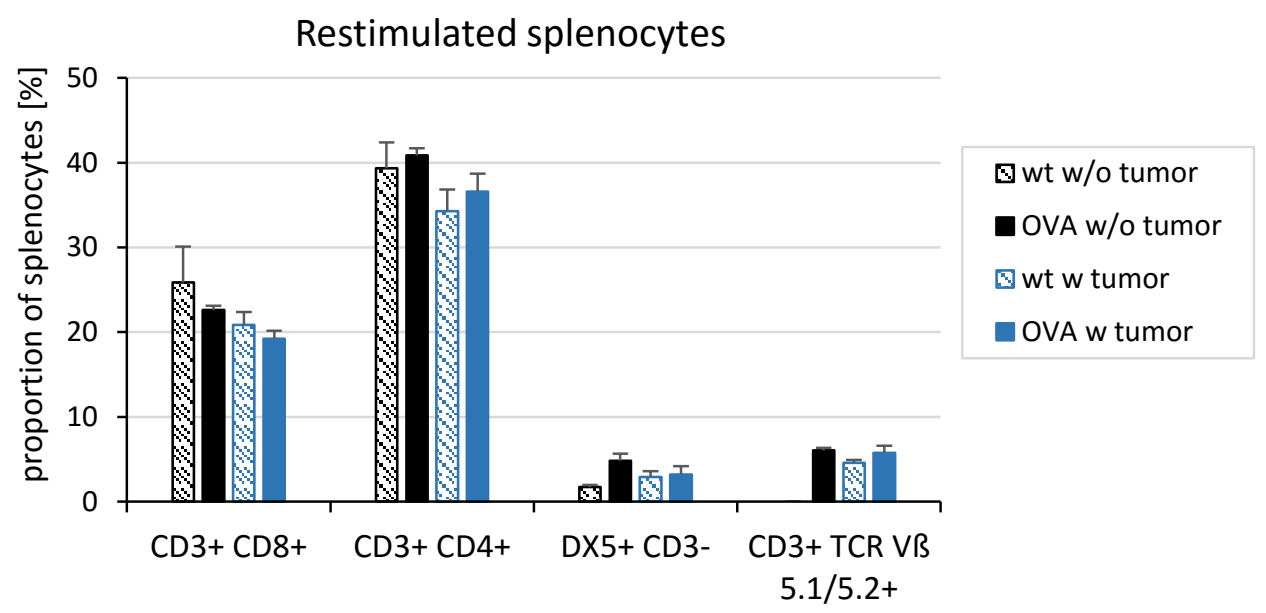

Figure 44 Flow cytometric analysis of restimulated splenocytes on day 4 after in vitro stimulation with OVA protein and IL-2.

Splenocytes were obtained from 129/Sv mice which previously received OVA-expressing (OVA) or wildtype (wt) stem cells, which led to tumor formation (w tumor) or tumor rejection (w/o tumor). Wt w/o tumor: $\mathrm{CD}^{+} \mathrm{CD}^{+}(n=7), \mathrm{CD}^{+} \mathrm{CD}^{+}(\mathrm{n}=7), \mathrm{DX5}^{+} \mathrm{CD}^{-}(\mathrm{n}=7) \mathrm{CD}^{+} \mathrm{TCRVB}^{+}(n=0)$, OVA w/o tumor: $\mathrm{CD}^{+} \mathrm{CD}^{+}(n=126), \mathrm{CD}^{+} \mathrm{CD}^{+}(\mathrm{n}=36), \mathrm{DX5}^{+} \mathrm{CD}^{-}(\mathrm{n}=58), \mathrm{CD}^{+} \mathrm{TCRVB}^{+}(\mathrm{n}=15)$, wt w tumor: $\mathrm{CD}^{+} \mathrm{CD}^{+}(\mathrm{n}=22), \mathrm{CD}^{+} \mathrm{CD}^{+}(\mathrm{n}=13), \mathrm{DX5}^{+} \mathrm{CD}^{-}(\mathrm{n}=22), \mathrm{CD}^{+} \mathrm{TCRV}^{+}(\mathrm{n}=6), \mathrm{OVA}^{-}$ w tumor: $\mathrm{CD}^{+} \mathrm{CD}^{+}(n=36), \mathrm{CD}^{+} \mathrm{CD}^{+}(\mathrm{n}=3), \mathrm{DX5}^{+} \mathrm{CD}^{-}(\mathrm{n}=5), \mathrm{CD}^{+} \mathrm{TCRVB}^{+}(\mathrm{n}=4)$.

The restimulated splenocytes were used as effector cells in ${ }^{51} \mathrm{Cr}$ release assays with two RMA target cells lines to examine the activity of OVA-specific CTLs in animals after injections with OVA-expressing 
stem cells or wildtype stem cells. The RMA target cells comprised of an OVA-expressing RMA cell line (RMA eGFP-OVA, here referred as RMA OVA), which was transfected with the transgene for a OVAeGFP fusion protein (as OVA-expressing stem cell lines) and a control cell line (RMA eGFP; here referred as RMA control), lacking the OVA in the transfected construct. In Figure 45, the specific lysis of those RMA target cell lines by splenocytes isolated of two animals is shown, of which one received wildtype cells (iPSC 129/Sv) and the other OVA-expressing stem cells (iPSC OVA\#13). The control RMA cell line was neither killed by splenocytes of the animal receiving OVA-expressing stem cells nor from splenocytes of the mouse that received wildtype stem cells. However, the OVA-expressing RMA target cell line was only lysed by splenocytes of recipients of OVA-expressing stem cells, indicating the presence of OVA-specific CTLs in this animal.

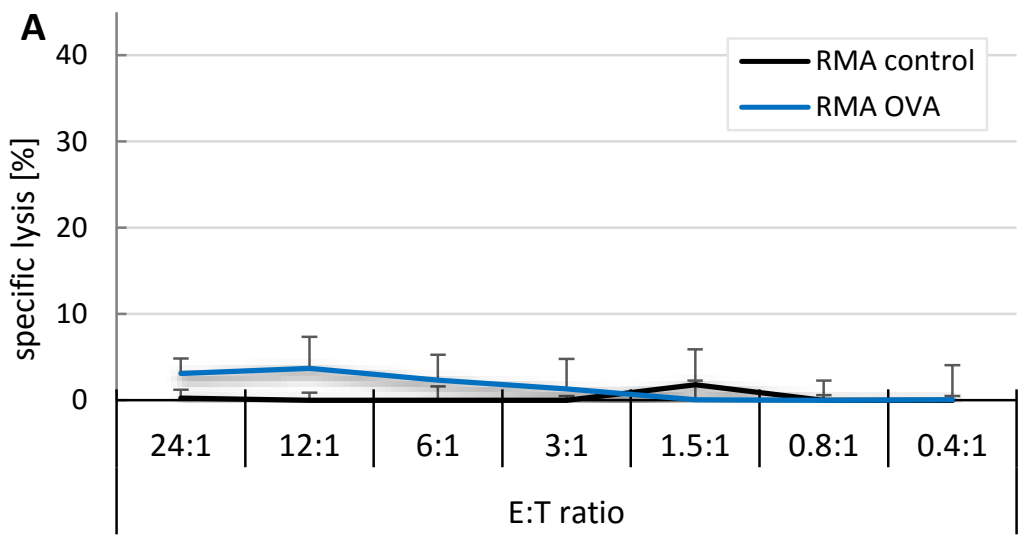

Figure 45 Analysis of OVAspecific CTLS in $129 / \mathrm{Sv}$ animals which received stem cell inoculations.

Shown is the specific lysis of control RMA (RMA eGFP) and RMA OVA (RMA eGFP-OVA) by in vitro restimulated splenocytes (day 4) isolated from animals, which received (A) wildtype or (B) OVAexpressing stem cells + SEM

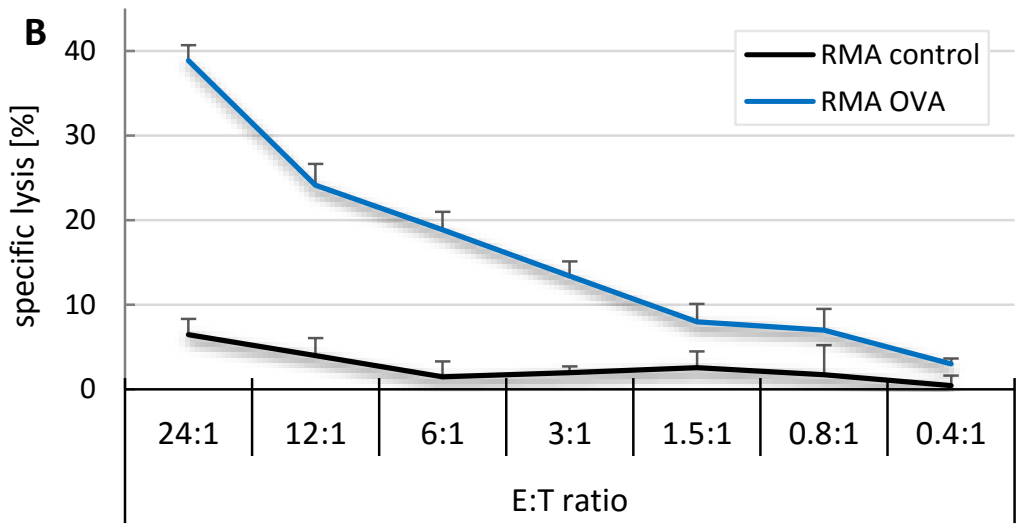

of experimental triplicates. (A) Splenocytes were obtained on day 49 after injection of iPSC $129 /$ Sv stem cells that led to tumor formation. (B) Splenocytes were obtained on day 82 after injection of iPSC $129 /$ Sv OVA\#13 cells, that did not form a teratoma in the mouse.

In Figure 46A, a summary of the specific lysis of OVA-expressing RMA and control RMA by splenocytes of animals which previously received either wildtype or OVA-expressing iPSCs (iPSC 129/Sv) or ESCs (ESC BTL1, ESC MPI-II) is shown. On average, the specific lysis of RMA OVA targets by splenocytes of animals which received OVA-expressing stem cells was higher (16\% for ESC OVA and $20 \%$ for iPSC OVA at the highest E:T ratio), than the lysis by splenocytes isolated of $129 /$ Sv hosts which received 
wildtype stem cells (6.4\% for ESC wt, $7.8 \%$ for iPSC wt). In Figure 46B, the lysis of RMA OVA by splenocytes derived from animals, which received the individual stem cell clones is depicted separately. Despite the fact that the specific lysis of RMA OVA by the various splenocytes differed, all animals which received OVA-expressing stem cells gave rise to OVA-specific CTLs.

A
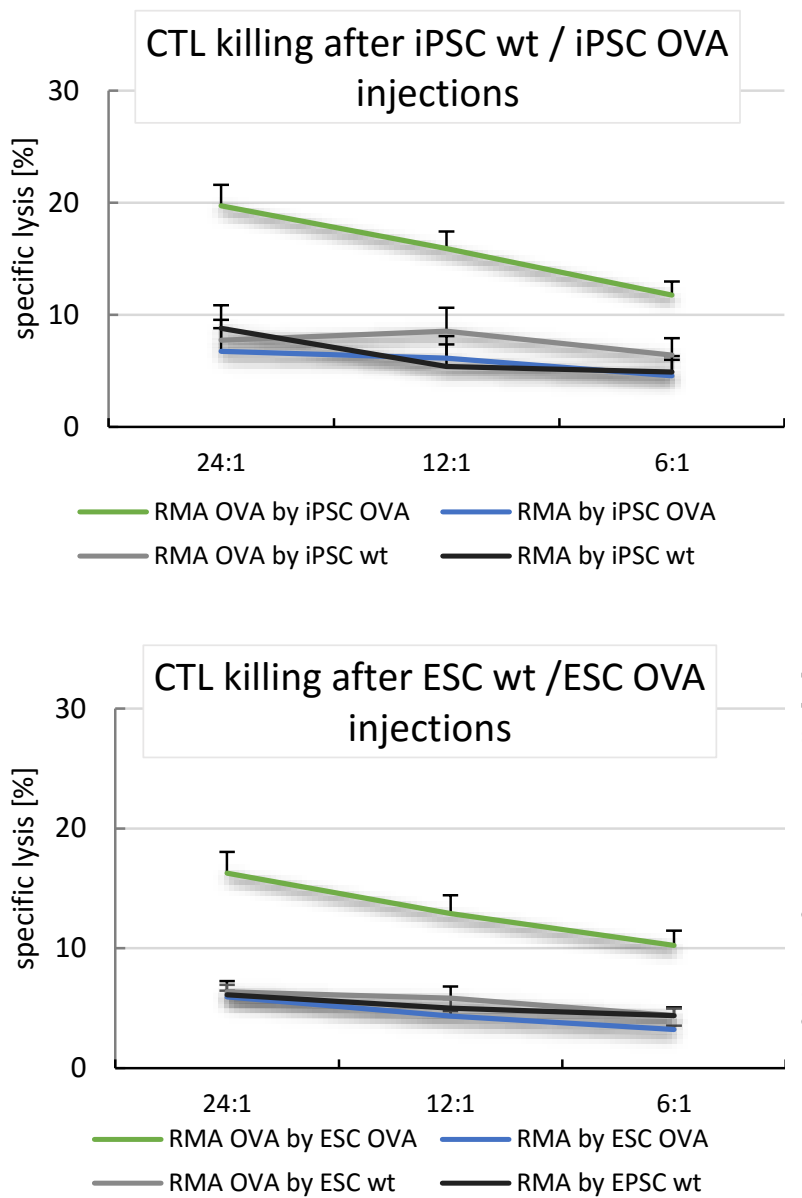

B
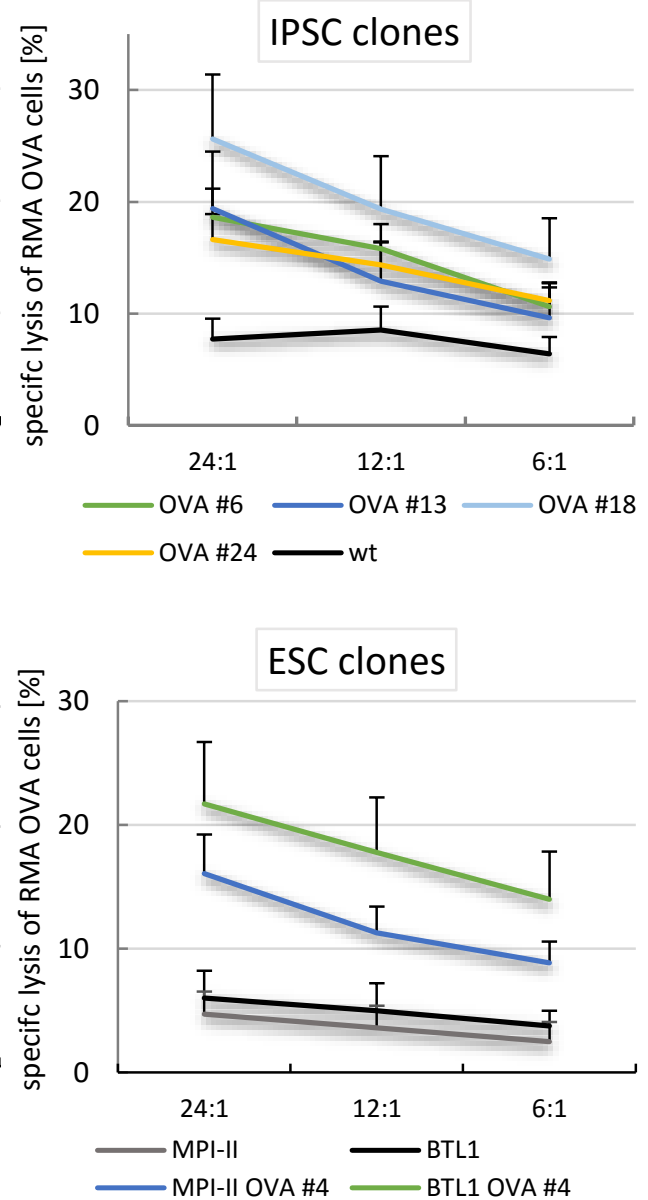

Figure 46 Analysis of the presence of OVA-specific T cells after stem cell inoculation in syngeneic hosts by ${ }^{51} \mathrm{Cr}$ release assays at the end point of the experiment (long-term).

(A) Shown is the mean specific lysis + SEM of RMA control cells (RMA eGFP) and RMA OVA (RMA eGFP-OVA) cells by splenocytes from syngeneic hosts inoculated with OVA-expressing or wildtype ESCs or iPSCs at different E:T ratios. IPSC OVA $(n=61)$, iPSC wt $(n=8)$, ESC OVA $(n=104)$, ESC wt $(n=28)$. Differences between the killing of control RMA and RMA OVA by various splenocytes of animals that received iPSC OVA $\left(p=6.7 \times 10^{-22}\right)$, iPSC wt $(p=0.39)$, ESC OVA $\left(p=3.5 \times 10^{-25}\right)$, ESC wt $(p=0.83)$; Mann-Whitney U-test. (B) Shown is the mean specific lysis + SEM of RMA OVA target cells by splenocytes of $129 / \mathrm{Sv}$ which received distinct ES and iPS cell clones. IPSC OVA\#6 $(n=19)$, iPSC OVA\#13 $(n=6)$, iPSC OVA\#18 $(n=15)$, iPSC OVA\#24 $(n=21)$, iPSC wt $(n=8)$, ESC BTL1 wt $(n=17)$, ESC BLT1 OVA\#4 $(n=27)$, ESC MPI-II wt $(n=3)$, ESC MPI-II OVA\#4 $(n=17)$. Differences between the killing of control RMA and RMA OVA by various splenocytes of animals that received iPSC OVA\#6 $\left(p=7.5 \times 10^{-9}\right)$, iPSC OVA\#13 $\left(p=3.5 \times 10^{-3}\right)$, iPSC OVA\#18 $\left(p=1.5 \times 10^{-5}\right)$, iPSC OVA\#24 $\left(p=3.9 \times 10^{-9}\right)$, iPSC wt $(p=0.39)$, ESC BTL1 wt $(p=0.47)$, ESC BLT1 OVA\#4 $\left(p=1.1 \times 10^{-9}\right)$, ESC MPI-II wt $(p=0.15)$, ESC MPI-II OVA\#4 $\left(p=5.1 \times 10^{-8}\right)$; Mann-Whitney U-test. 
Comparative analysis revealed that the specific lysis of OVA-expressing targets was higher by splenocytes of animals which did not show tumor formation ( $23 \%$ ) after OVA-expressing stem cell inoculation compared to lysis by splenocytes of animals which received the same stem cells but displayed tumor formation (14.6\%, $p=9.1 \times 10^{-6}$, Mann Whitney U-test) (Figure 47A). After inoculation of wildtype stem cells, these differences were not monitored. This suggests that increased OVAspecific CTL activity led to tumor rejection in animals which received OVA-expressing stem cells.
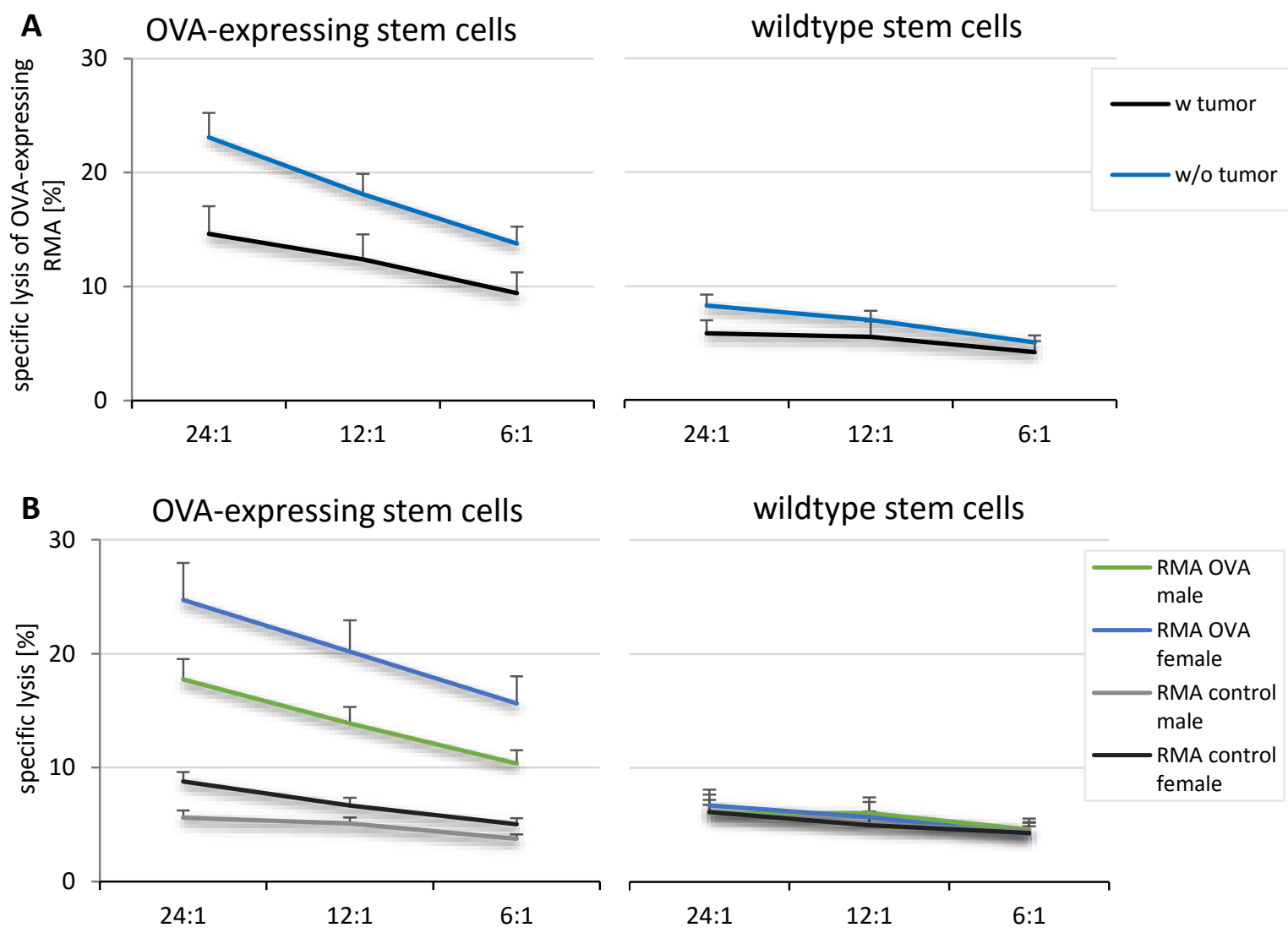

Figure 47 Analysis of OVA-specific CTL responses dependent on (A) tumor formation or (B) gender of hosts. (A) Shown is the mean specific lysis of OVA-expressing RMA targets by splenocytes of animals which received OVA-expressing stem cells (left) or wildtype stem cells (right) and which showed tumor formation ( $w$ tumor) or no tumor formation (w/o tumor) + SEM determined by ${ }^{51} \mathrm{Cr}$ release assays. OVA-expressing stem cells: $w$ tumor $(n=35)$, w/o tumor $(n=78)$, wildtype stem cells: $w$ tumor $(n=22)$, w/o tumor $(n=6)$. Differences w/o tumor vs. w tumor: OVA-expressing stem cells $\left(p=9.1 \times 10^{-6}\right)$, wt stem cells $(p=0.06)$.(B) Influence of gender of hosts which received stem cell inoculations on the lysis rate of RMA target cell lines. Shown is the mean specific lysis of control RMA and RMA OVA by splenocytes obtained from male and female mice which received OVA-expressing stem cells (left) or wildtype stem cells (right) + SEM determined by ${ }^{51} \mathrm{Cr}$ release assays. OVAexpressing stem cells: male $(n=69)$, female $(n=44)$, wildtype stem cells: male $(n=14)$, female $(n=14)$. 
Noticeable, after inoculation of OVA-expressing stem cells, the specific lysis of the OVA-expressing target cell line by splenocytes of female host was significantly higher than the lysis by splenocytes obtained from male hosts (RMA OVA m vs. f: $p=2.9 \times 10^{-5}$; Mann-Whitney U-test; Figure 47B). Inoculation of wildtype stem cells did not led to any differences in killing of RMA OVA and control RMA by splenocytes obtained from male or female hosts (RMA control $m$ vs. $f: p=0.97$; RMA OVA $m$ vs. $f: p=0.67 ;$ Mann-Whitney U-test). This indicates that that female recipients develop stronger immune responses against OVA.

Next, the presence of an OVA-specific CTL activity was analyzed in animals receiving injections of OVAexpressing stem cell clones, which were compromised in their pluripotency and did not form teratomas, or only in a minority of experiments, in immunodeficient hosts. As seen in Figure 48 , the not fully pluripotent cell clones elicited a CTL response against OVA. The stem cell clones, which never gave rise to a teratoma in immunodeficient hosts, also induced OVA-specific CTLs but at a much lower level. This suggest that OVA-expressing cells derived from stem cells in vivo contribute to the CTL response. Injections of OVA-expressing stem cells, which lost the ability to substantially proliferate and differentiate in vivo elicited only a minor CTL response.

OVA-specific CTLS

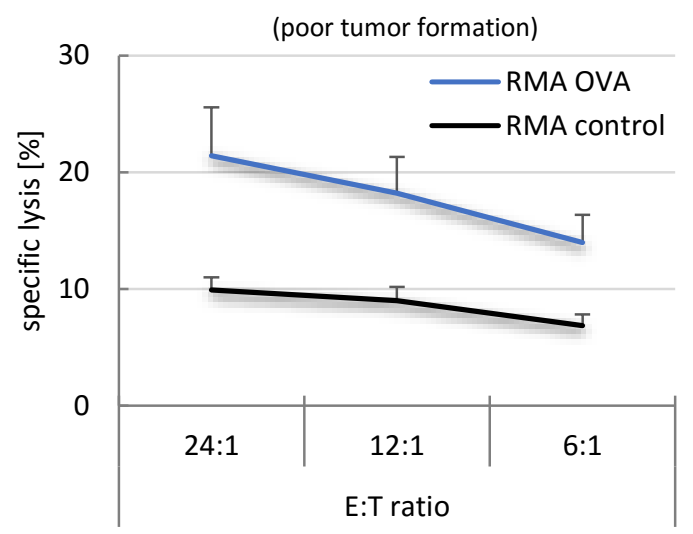

OVA-specific CTL

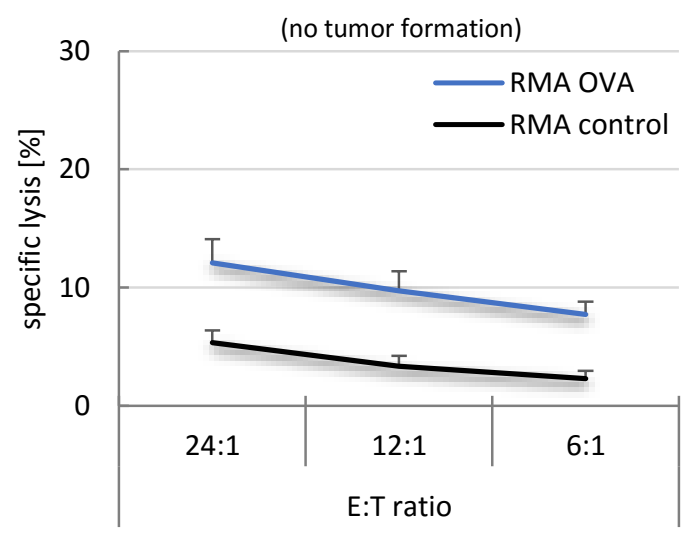

Figure 48 Analysis of the presence of OVA-specific T cells in syngeneic 129/Sv animals after stem cell inoculation.

Splenocytes of $129 / \mathrm{Sv}$ were used as effector cells in ${ }^{51} \mathrm{Cr}$ release assays with control RMA and RMA OVA as target cells. Animals received injections of stem cell clones, which were considered as not fully (left side) or not pluripotent (right side) due to reduced or lacking tumor formation in immunodeficient hosts. Shown is the mean specific lysis of RMA control (RMA-eGFP) and RMA OVA (eGFP-OVA) + SEM. Poor tumor formation: ESC BTL1 OVA\#1, ESC BTL1 OVA\#20 ( $n=15)$; differences in the killing of RMA OVA and control RMA $\left(p=6.7 \times 10^{-9}\right)$, Mann-Whitney U-test; no tumor formation :ESC BTL1 OVA\#9, ESC BTL1 OVA\#14, ESC MPI-II OVA\#1 $(n=31)$, differences in the killing of RMA OVA and control RMA $\left(p=2.3 \times 10^{-5}\right)$, Mann-Whitney U-test. 
To determine when after inoculation of stem cells a CTL response was induced, short-term experiments were performed, in which animals were sacrificed one week after injections of wildtype or OVA-expressing stem cells. In Figure 49, the specific lysis of OVA-expressing RMA and control RMA by splenocytes of animals which previously received either iPSC 129/SV or ESC BTL1 wildtype or OVAexpressing stem cells or no cells as control animals is depicted.
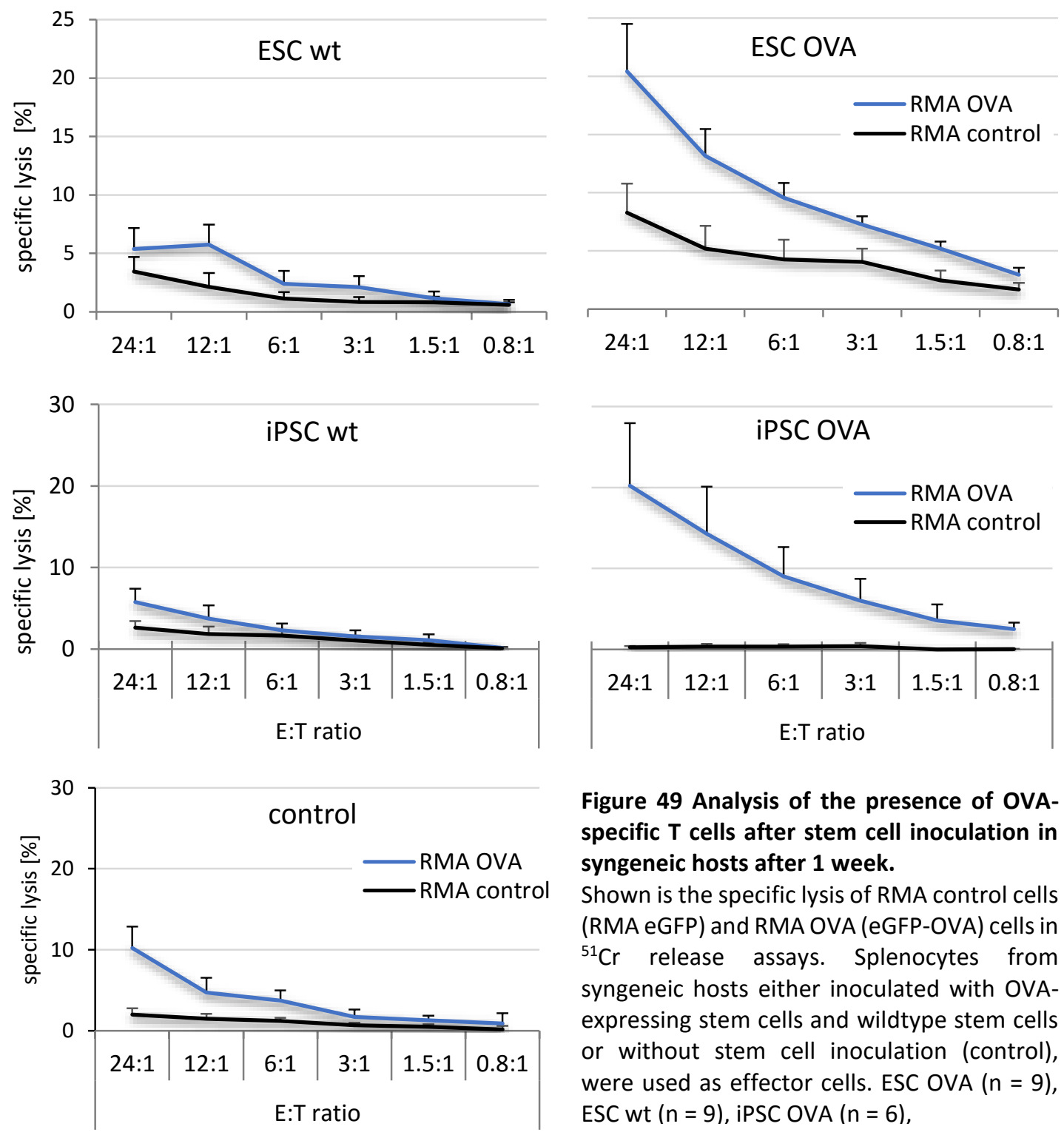

Figure 49 Analysis of the presence of OVAspecific $T$ cells after stem cell inoculation in syngeneic hosts after 1 week.

Shown is the specific lysis of RMA control cells (RMA eGFP) and RMA OVA (eGFP-OVA) cells in ${ }^{51} \mathrm{Cr}$ release assays. Splenocytes from syngeneic hosts either inoculated with OVAexpressing stem cells and wildtype stem cells or without stem cell inoculation (control), were used as effector cells. ESC OVA $(n=9)$, ESC wt $(n=9)$, iPSC OVA $(n=6)$,

iPSC wt $(n=8)$, control $(n=9)$. Comparison of RMA an RMA OVA killing: ESC wt $(p=0.58)$, ESC OVA $\left(p=2.6 \times 10^{-6}\right)$, iPSC wt $(p=0.08)$, iPSC OVA $\left(p=7.8 \times 10^{-11}\right)$; Mann-Whitney U-test.

The splenocytes of animals, which were injected with OVA-expressing stem cells showed a higher lysis rate of the OVA-expressing target cell line than their wildtype counterparts and the control. On 
average, specific lysis of control RMA at the highest E:T ratio ranged between $5.4 \%$ (ESC BTL1 wt), $5.8 \%$ (iPSC 129/Sv wt), $10.2 \%$ (control), whereas up to $20.2 \%$ (ESC BTL1 OVA) or $20.4 \%$ (iPSC 129/Sv OVA) of the targets were killed when OVA-expressing stem cells had been inoculated. Thus, the lysis of both targets cell lines by splenocytes only significantly differed if animals previously received OVAexpressing stem cells. This shows that the OVA-specific CTLs were present already one week after transplantation of the stem cells.

\subsubsection{Killing of wildtype and OVA-expressing iPSCs and ESCs by NK cells}

In parallel to several of the ${ }^{51} \mathrm{Cr}$ release assays in which control RMA and RMA OVA were used as targets to determine CTL response against OVA in long term experiments, YAC-1, which is highly sensitive to NK cells, was used as target cell line. In this way the activation of NK cells in animals which either received OVA-expressing stem cells or wildtype stem cells or of animals which gave rise to teratomas versus those which did not show tumor formation was analyzed. The lysis of YAC-1 by splenocytes of animals which received OVA-expressing stem cells was higher than the killing by splenocytes of mice that had received wildtype stem cells (Figure 50, left panel). Moreover, the NK cell activity was higher in animals without tumors than in animals with tumors (Figure 50, right panel). These results indicate that in parallel to a CTL response against OVA, the NK cell activity increased in these mice. This might contribute to tumor rejection as suggested by the higher NK cell activity in the tumor-free mice.

NK cell killing
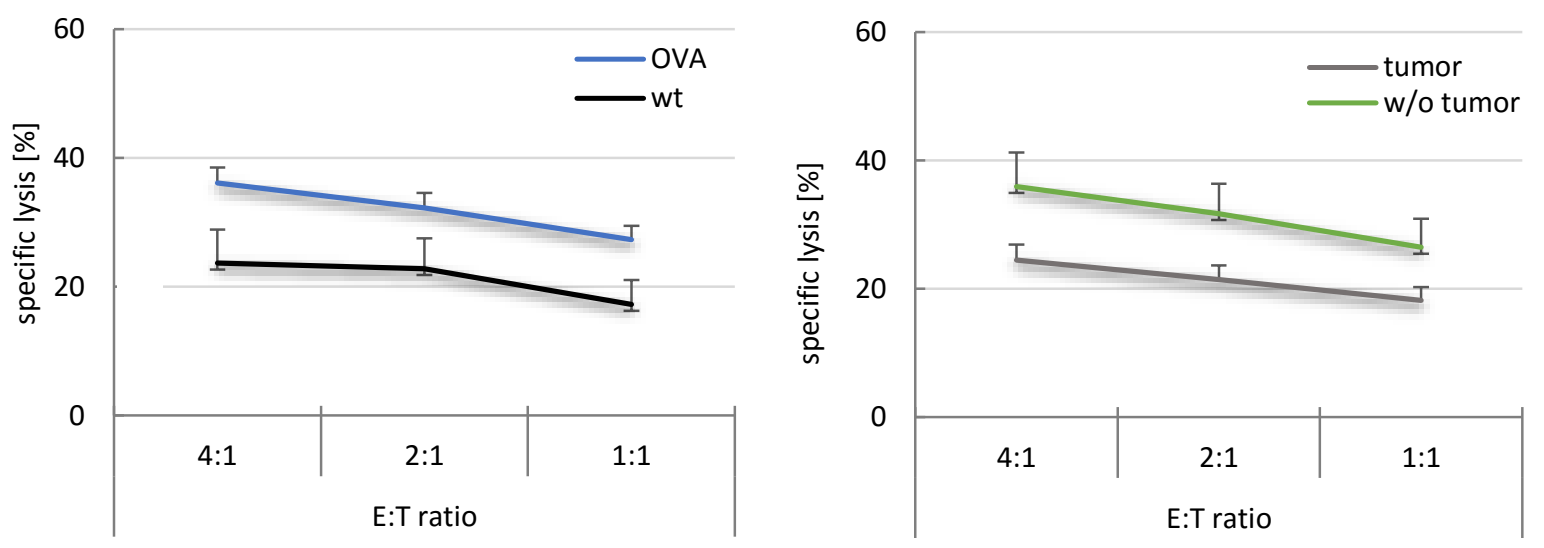

Figure 50 Analysis of NK cell activity in animals which either received wildtype (wt) or OVA-expressing (OVA) stem cells, either leading to tumor formation (tumor) or tumor rejection (w/o tumor).

Splenocytes were used in ${ }^{51} \mathrm{Cr}$ release assays as effector cells against the target cell line YAC-1, which is known to be highly sensitive to NK cell lysis. Shown is the mean specific lysis of YAC-1 cells + SEM at different effector (CD3DX5 $)$ to target ratios. OVA $(n=65)$, wt $(n=14)$, tumor $(n=14)$, w/o tumor $(n=65)$. OVA vs. wt $\left(p=8.1 \times 10^{-4}\right)$, tumor vs. w/o tumor $\left(p=2.4 \times 10^{-3}\right)$; Mann-Whitney U-test. 


\subsubsection{OVA-specific antibody generation in syngeneic hosts}

Whether the expression of OVA in stem cells elicited an antibody response in otherwise syngeneic hosts was analyzed via ELISA. For this, sera of animals which previously received injections with OVAexpressing stem cells, wildtype cells or no cells were collected and analyzed to determine OVA-specific antibodies by measuring ODs at $405 \mathrm{~nm}$ at several different serum dilutions (1:20 to 1:2560 for general anti-OVA titer, 1:30 to 1:240 for isotype subclasses). For the determination of the general OVA titer a secondary antibody reacting with IgG and IgM antibodies was used, for which a cross-reactivity to further antibody classes was not excluded. As positive control, four 129/Sv mice were immunized with OVA protein and TiterMax adjuvant and further boosting injections with OVA protein. These control sera were pooled, stored in aliquots and used as reference serum on each ELISA plate by setting ODs at the lowest dilution (1:20) to $100 \%$ to calculate the relative OVA antibody titer of other sera. Sera were obtained from blood samples after animals were sacrificed at experimental end points. In the long-term setting, the duration of the experiments varied between single animals depending on tumor size and animal condition but ranged from 21 (w tumor) to 90 (w/o tumor) days. In Figure 51A it is shown that OVA-specific antibodies were found in the sera of animals which received OVA-expressing stem cells. Hereby, the average relative OVA titer ( $94 \%)$ was similar to the OVA titer of the control immune serum (100\%). In contrast, the average relative anti-OVA titer of animals which received wildtype stem cells $(27 \%)$ was on a similar level as of those animals which were not injected with any cells (34\%). Analysis of sera of animals which were either injected with ESCs or iPSCs showed that OVA expression in both ESCs and iPSCs led to significant higher relative anti-OVA titers (ESC wt vs. ESC OVA: $p=5.6 \times 10^{-3}$; iPSC wt vs. iPSC OVA: $p=7.5 \times 10^{-7}$, Mann-Whitney U-test) (Figure 51B). However, relative OVA titers were higher in mice that have received OVA-expressing iPSCs than ESCs (ESC OVA vs. iPSC OVA: $p=2.3 \times 10^{-10}$; ESC wt vs. iPSC wt: $p=2.1 \times 10^{-17}$, Mann-Whitney U-test). Significant variations in OVA titers occurred dependent on the injected clones as depicted in Figure 51C, e.g. showing anti-OVA antibody titers up to $153 \%$ relative to control titers after injection of iPSC OVA clones \#6 and \#24. ESC OVA clone injections led to relative anti-OVA titers of $61 \%$ (BTL OVA\#4) to $90 \%$ (MPI OVA\#4), whereas injections of iPSC OVA\#13 caused anti-OVA titers similar to those found after wildtype iPSC injections. Moreover, it was analyzed whether animals which rejected tumors showed divergent anti-OVA titers compared to animals showing tumor formation. Anti-OVA antibodies showed significant higher titers in animals that formed teratomas compared to those without tumors, whereas the OVA antibody level after wildtype stem cell injection was independent 
of tumor formation or rejection (Figure 51D). Furthermore, it was analyzed whether gender of hosts had influence on the generation of OVA antibodies, but no dependency was found.

\section{Anti-OVA titer}
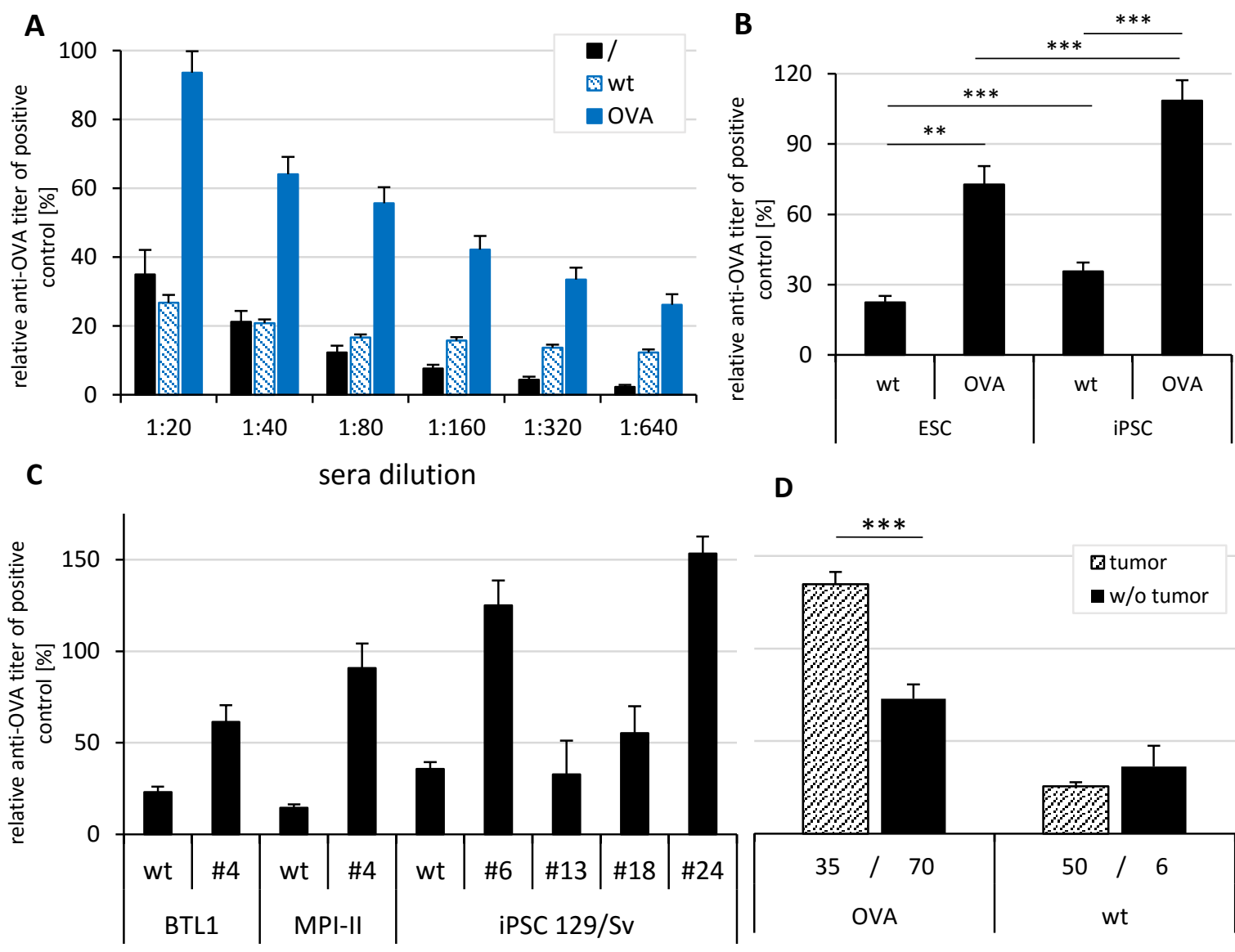

D

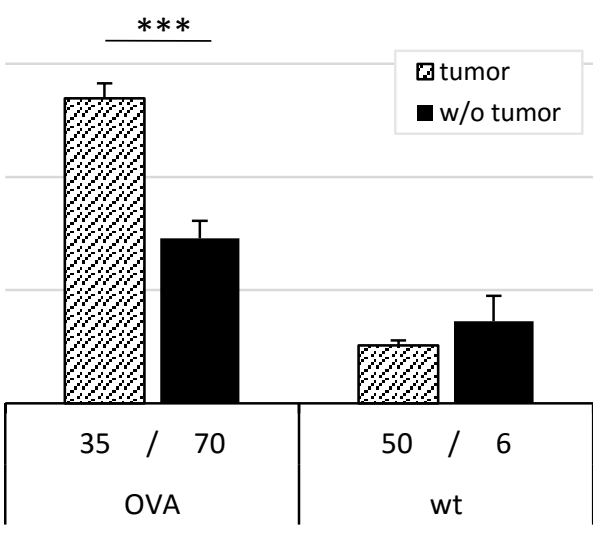

Figure 51 Analysis of OVA-specific antibody titers in syngeneic 129/Sv mice after injections of wildtype or OVA- expressing stem cells via ELISA.

Sera were obtained from blood samples at the experimental end points. Shown is the mean relative anti-OVA titer + SEM compared to a pool of positive controls, which were previously immunized with recombinant OVA protein. (A) Mean relative anti-OVA titer in animals which received either no cell injections (/) or wildtype (wt) or OVA-expressing stem cells (OVA), depicted in serial dilutions. Control (/) $(n=12)$; wt $(n=56)$; OVA $(n=105)$. (B) Comparison of mean relative anti-OVA titer for the smallest sera dilution (1:20) of animals which received either wildtype or transgenic ESCS or iPSCs: ESC OVA $(n=44)$, ESC wt $(n=38)$, iPSC OVA $(n=61)$, iPSC wt $(n=$ 18). ${ }^{* *}<0.01,{ }^{* * *}<0.001$; Mann-Whitney U-test. (C) Comparison of relative anti-OVA titers after injections of distinct stem cell clones in 129/Sv mice. ESC BTL1 wt $(n=35)$, ESC BTL1 OVA\#4 $(n=27)$, ESC MPI-II wt $(n=3)$, ESC MPI-II OVA\#4 ( $n=17)$, iPSC 129/Sv wt $(n=18)$, iPSC 129/Sv OVA\#6 $(n=19)$, iPSC 129/Sv OVA\#13 $(n=6)$, iPSC 129/SV OVA\#18 ( $n=15)$, iPSC 129/Sv OVA\#24 ( $n=21)$. (D) Anti-OVA antibody titers in animals which showed tumor formation or rejected tumors after stem cell inoculation of wildtype or OVA-expressing stem cells. Numbers of performed experiments are depicted below each bar. Differences in OVA titers between animals showing tumor formation or not: OVA $\left(p=2.8 \times 10^{-31}\right)$, wt $(p=0.1)$, Mann-Whitney U-test. 
If OVA-antibodies (relative anti-OVA titer $>60 \%$ ) were found, the sera were additionally analyzed for the isotypes of OVA-specific antibodies using isotype-specific secondary antibodies in ELISAs. This showed that in animals which had received OVA-expressing stem cells, the OVA-specific IgG1 titer was increased compared to sera of animals which did not receive stem cell injections (Figure 52). Furthermore, OVA-specific antibodies of the IgG2a and IgG2b isotypes were found. No OVA-specific antibodies were found among the other isotypes. These findings indicate that the OVA-specific antibodies which arose after transplantation of OVA-expressing stem cells were of the IgG isotype. Moreover, this demonstrates that a class switch occurred in OVA-specific B cells.

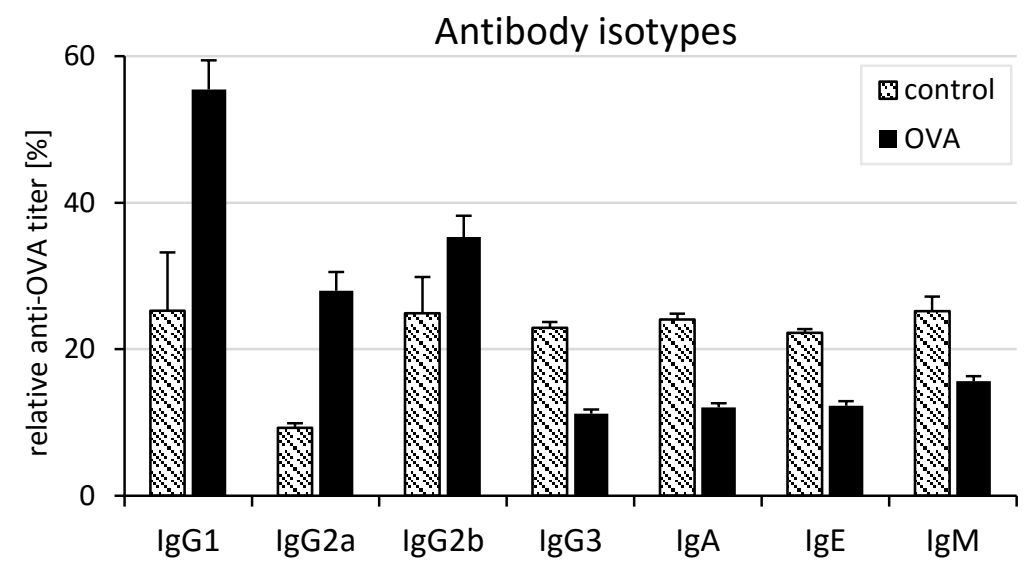

Figure 52 Analysis of OVA antibody isotype distribution in sera of $129 / \mathrm{Sv}$ mice which received no stem cells (control) or OVA-expressing stem cells. (OVA).

Shown is the mean proportion of various OVA-specific antibody isotypes in the sera $(1: 30)$ obtained after experimental endings + SEM. OVA $(n=79)$, control $(n=3)$.

In short-term experiments, in which animals were sacrificed 1 week after stem cell inoculation, the antibody response against OVA was also examined. Comparison of anti-OVA antibody levels of animals which either received wildtype stem cells (ESC BTL1 wt and iPSC 129/Sv wt), OVA-expressing stem cells (ESC BTL1 OVA\#4 and iPSC 129/Sv OVA\#6) and control animals (no cell injections) showed no differences in OVA-specific antibody titers ( $p=0.34$, Kruskal-Wallis H-test) one week after injections (Figure 53A). Examination of an anti-OVA antibody isotype distribution as depicted in Figure 53B showed no major differences compared to controls. In general, no increased levels of OVA-specific antibodies were found 1 week after inoculation of OVA-expressing stem cells compared to control animals and animals which received wildtype stem cells. 
A

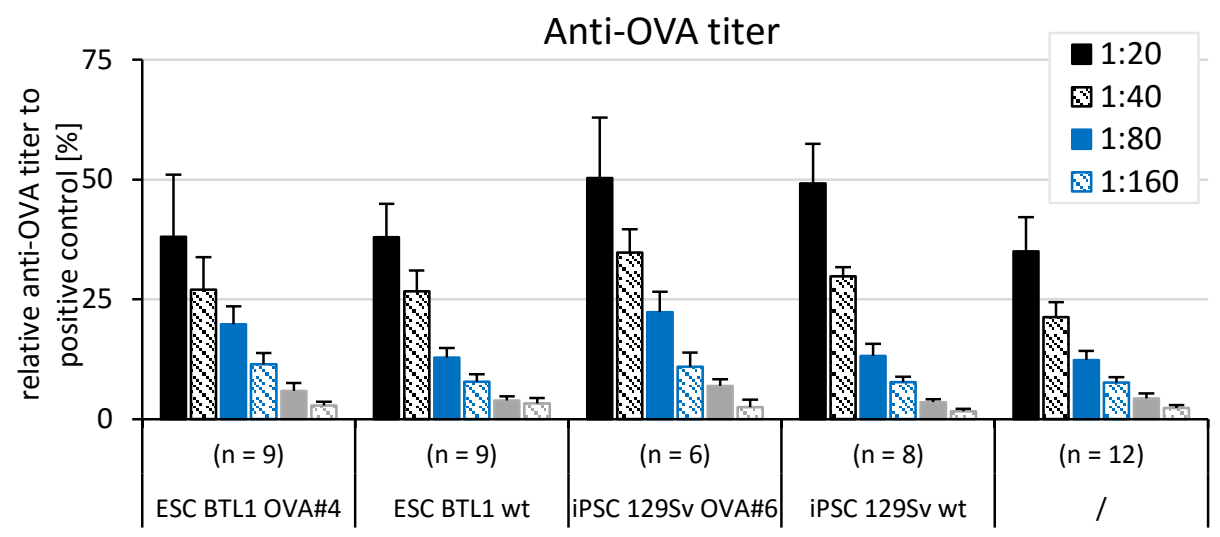

B

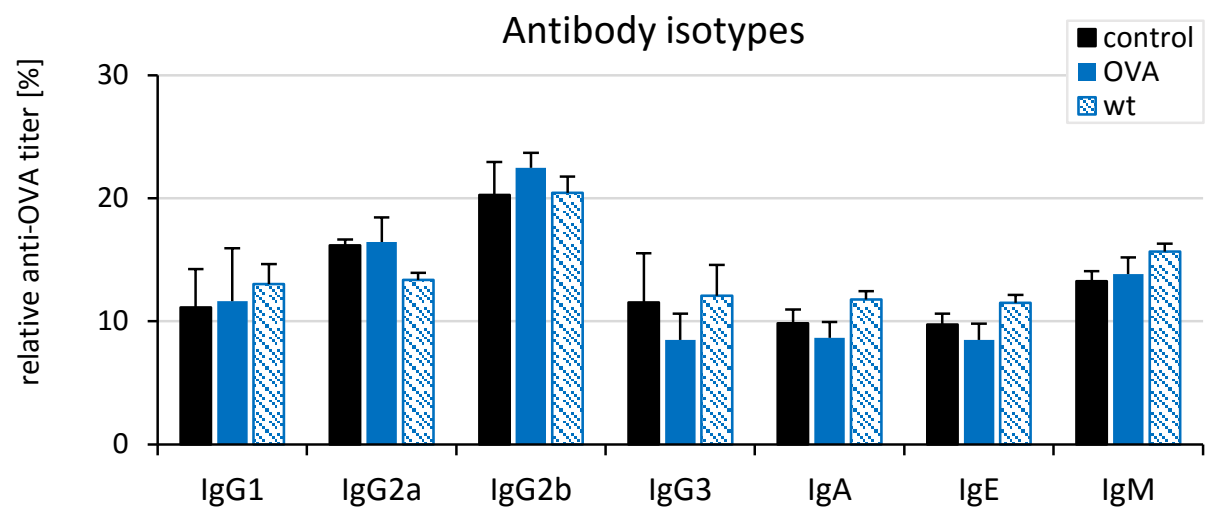

Figure 53 Analysis of OVA-specific antibody titer in syngeneic hosts 1 week after stem cell injections. (A) Mean of relative anti-OVA titer after various cell injections + SEM in serial dilutions from 1:20 to 1:320 is depicted. As positive control for normalization sera of four 129/Sv mice immunized with recombinant OVA protein was used. Numbers of analyzed sera are depicted below columns. (B) Mean proportion of antibody isotypes of OVA antibodies at a dilution of 1:30 in various sera + SEM are shown. Control $(n=4)$, OVA $(n=7)$, wt $(n=9)$.

\subsubsection{Summary}

Analysis of OVA-expressing and wildtype stem cells in vivo showed that the expression of OVA as a model of a miHC antigen had effects on the engraftment of stem cells in immunocompetent syngeneic hosts, affecting tumor frequency, tumor size and weight. Additionally, it was shown that the injection of OVA-expressing stem cells elicited OVA-specific immune responses including CTL responses and induced OVA-specific IgG antibodies, demonstrating also class switching of OVA-specific B cells. CTL responses against OVA were already found early after stem cell injection (1 week), whereas antibody generation was monitored only later after injection, with higher OVA-specific titers in animals which showed a tumor formation. Moreover, NK cells might be involved in the rejection of stem cells. 
Adoptive transfer experiments revealed that OVA-specific T cells alone were able to delay tumor growth in immunodeficient hosts, but were not able to completely suppress tumor formation. Generally, all teratomas were infiltrated by various immune cells. The presence of immune escape mechanisms or immunogenicity marker expression on stem cells and their derivatives could not be confirmed in this study. In conclusion, the expression of a single antigen largely impaired engraftment of stem cells in immunocompetent recipients.

\subsection{The role of NKG2D in the killing of pluripotent stem cells}

In the previous experiments, not only an activation of CTLs but also of NK cells was observed. An activation of NK cells might be beneficial in the context of a transplantation of stem cell-derived grafts because PSC-derived grafts are at risk of giving rise to teratomas in the recipient, if residuals of undifferentiated cells remain in the grafts after in vitro differentiation. Our group previously showed that NK cells can kill allogeneic and autologous PSCs and reduce the risk of teratoma growth after transplantation of PSCs (Dressel et al., 2010; Kruse, Hamann et al., 2015). Inhibition studies indicated that NKG2D is an activating NK cell receptor which is involved in the killing of murine PSCs (Dressel et al., 2010), whereas for killing of human iPSCs DNAM-1 appeared to be more important (Kruse, Hamann et al., 2015). To further clarify the role of NKG2D in the murine system, the capability of NK cells from wild type C57BL/6 and NKG2D-deficient mice to kill various murine PSC lines was compared.

\subsubsection{Killing of murine stem cells by naïve C57BL/6 and NKG2D-deficient NK cells}

Six murine stem cell lines of different origin (iPSCs, ESCs, maGSCs) which were either syngeneic to the used NK cells (C57BL/6) or MHC-matched but otherwise allogeneic (129/Sv) were used as targets for freshly isolated wildtype C57BL/6 or NKG2D-deficient NK cells in ${ }^{51} \mathrm{Cr}$ release assays. The NK cellsensitive cell line YAC-1 was always included in the experiments as reference target cell line. Comparative analysis showed that all tested stem cell lines were moderately killed by freshly isolated wildtype C57BL/6 NK cells. The specific lysis at the highest E:T ratio ranged between $12.5 \%$ for iPSCS to $20.6 \%$ for maGSCs (Figure 54A). In contrast, the stem cells were largely resistant to killing by naïve NKG2D-deficient NK cells, showing specific lysis on the highest E:T ratio only between $3.9 \%$ for ESCs to $4.2 \%$ for iPSCs (Figure 54B). The killing of the stem cell lines and the NK cell sensitive cell line YAC1 by the NKG2D-deficient NK cells was significantly lower than killing by the wildtype C57BL/6 NK cells 
(ESCs: $p=3.4 \times 10^{-8} ;$ iPSCs: $p=8.4 \times 10^{-8} ;$ maGSCs: $p=7.7 \times 10^{-7} ;$ YAC-1: $p=5.2 \times 10^{-14} ; 2$-way-ANOVA adjusted for E:T ratio).
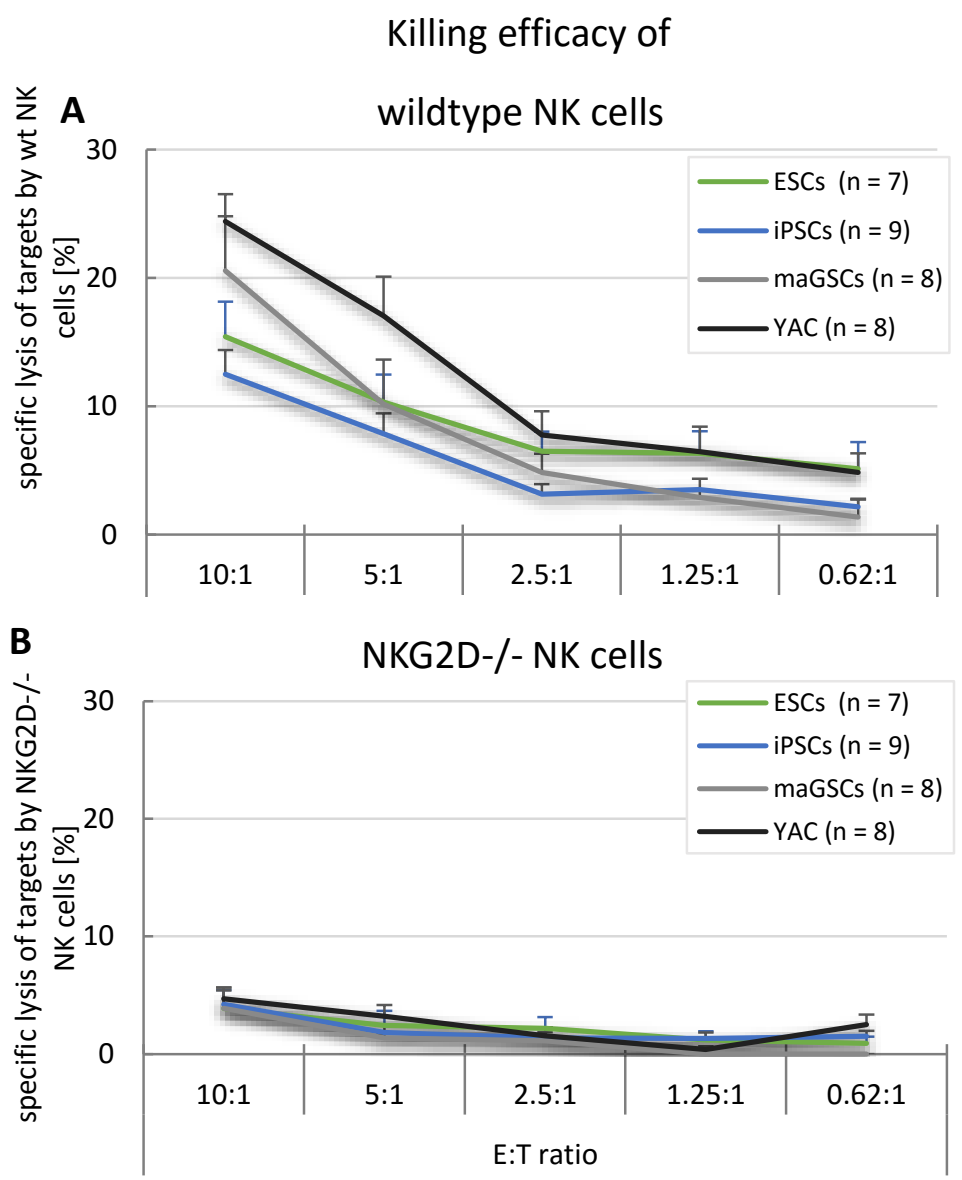

Figure 54 Killing of different stem cell lines and control YAC-1 cells by (A) naïve wildtype C57BL/6 NK cells or (B) NKG2D-deficient NK cells. Shown is the mean specific lysis of target cells + SEM. Differences between the killing efficiency of the two types of NK cells were significant for each type of cell. ESCs: ESCMPI-II and ESC BTL1; iPSCs: iPSC 129/Sv and iPSC C57BL/6; maGSCs: maGSCs $129 / \mathrm{Sv}$ and maGSCs C57BL/6.

The killing pattern of syngeneic (C57BL/6) or allogeneic but MHC-matched (129/Sv) target cells were similar. A significant lower killing off all six stem cell lines by NKG2D-deficient compared to the killing by wildtype C57BL/6 NK cells was observed (ESC BTL-1: $p=1 \times 10^{-4}$; ESC MPI-II: $p=8.7 \times 10^{-5}$, iPSC 129/Sv: $p=3.1 \times 10^{-7} ;$ iPSC C57BL/6: $p=0.01 ;$ maGSCs 129/Sv: $p=0.01 ;$ maGSCs C57BL/6: $p=1.1 \times 10^{-5} ;$ Figure 55). 


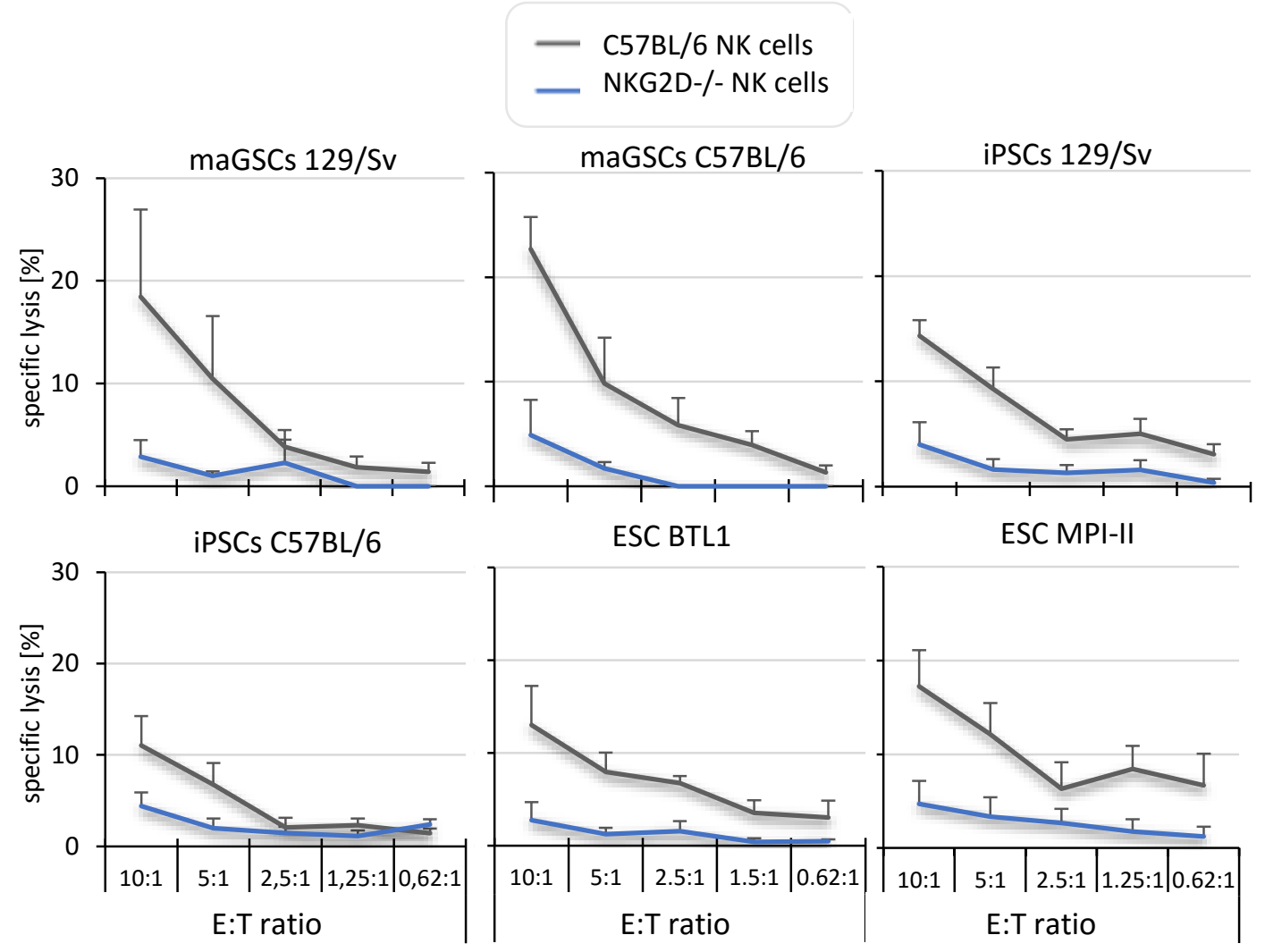

Figure 55 Killing of various stem cells lines by naïve wildtype C57BL/6 and NKG2D-deficient NK cells.

Shown is the mean specific lysis of stem cells by NK cells + SEM. Number of performed experiments: maGSCs 129/Sv $(n=4)$, maGSC C57BL/6 $(n=4)$, iPSC 129/Sv $(n=4)$, iPSC C57BL/6 $(n=5)$, ESC BTL1 $(n=3), \operatorname{ESC~MPI-II~}(n=4)$.

\subsubsection{Killing of murine stem cells by IL-2-activated wildtype C57BL/6 and NKG2D-deficient NK cells}

In the next experiments, the lysis of the different stem cell lines by in vitro IL-2-activated wildtype and NKG2D-deficient NK cells was compared. For this, isolated NK cells were in vitro stimulated for 4 days before they were used in cytotoxicity assays to determine their killing capacity of target cell lines. The specific lysis at the highest E:T ratio ranged between $22.8 \%$ for iPSCs to $40.3 \%$ for maGSCs, showing that the stimulation of the NK cells by IL-2 increased the killing of all tested stem cell lines (Figure 56). However, the NKG2D-deficiency was not fully compensated and wildtype NK cells were still more efficient than NKG2D-deficient NK cells, since the specific lysis on the highest E:T ratio by C57BL/6 NK cells ranged between $34.2 \%$ for iPSCs to $55.6 \%$ for maGSCs. This difference between the NK cell types was significant for iPSCs $(p=0.02)$ and maGSCs $\left(p=1.9 \times 10^{-5}\right)$ and at borderline for ESCs $(p=0.06)$. 


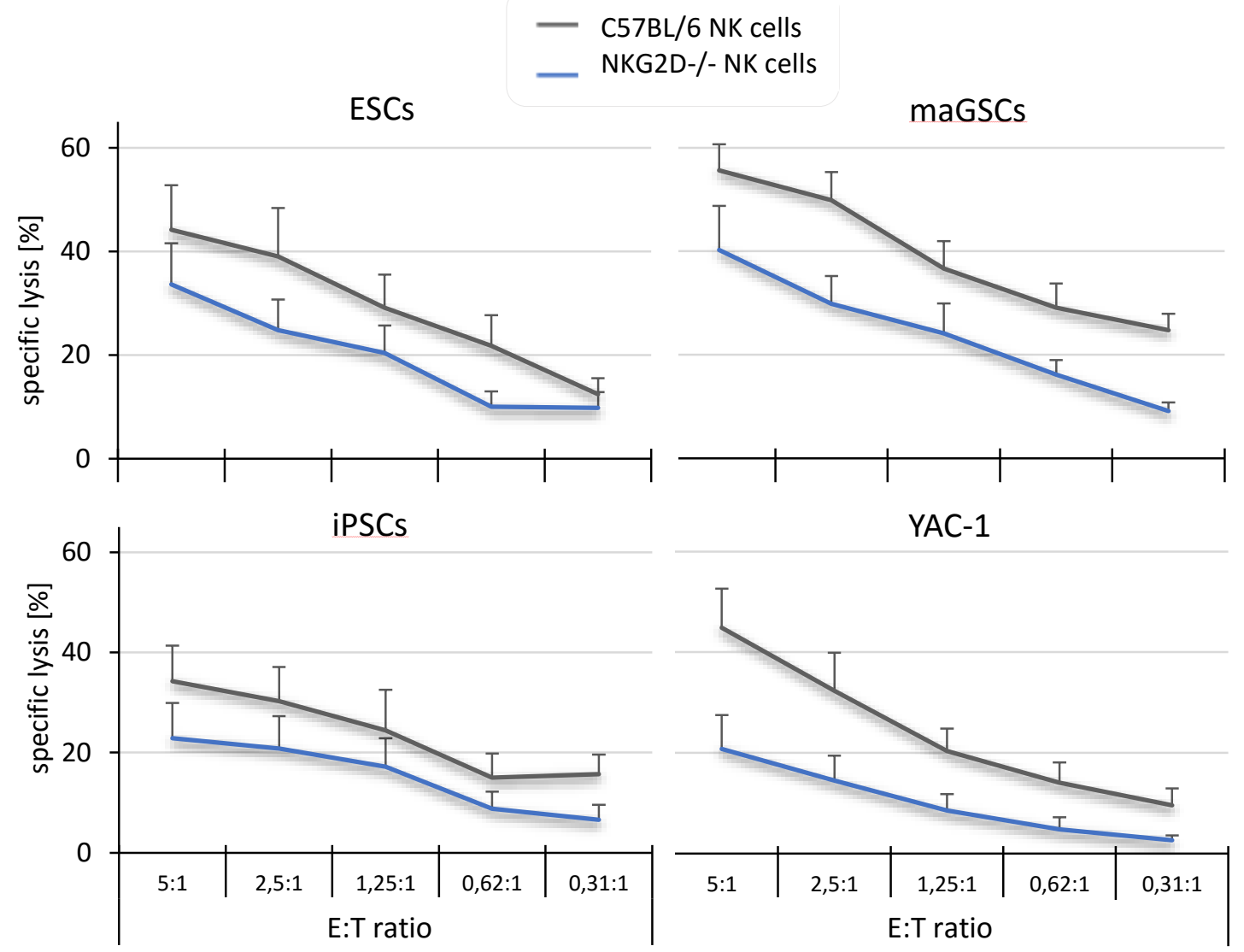

Figure 56 Killing of various cell lines by IL-2 stimulated wildtype C57BL/6 and NKG2D-deficient NK cells. NK cells were in vitro stimulated for 4 days before they were used in cytotoxicity assays to determine their killing capacity of target cell lines. Shown is the mean specific lysis + SEM. ESCs $(n=6)$, maGSCs ( $n=$ $6)$, iPSC ( $n=6)$, YAC-1 ( $n=6)$.

The lysis of the syngeneic (C57BL/6) or allogeneic but MHC-matched (129/Sv) stem cells is shown in Figure 57 for each stem cell line. Here, the difference between the lysis by wildtype and NKG2D ${ }^{-1}$ NK cells was significant for ESC BTL1 $\left(p=7.1 \times 10^{-4}\right.$ ) and both maGSC cell lines (maGSC 129/Sv: $p=0.01$; maGSC C57BL/6: $\left.p=5.9 \times 10^{-4}\right)$. The killing of the ESC MPI-II cell line by NKG2D-deficient NK cells after IL-2 stimulation was almost identical to the lysis rate by the wildtype NK cells $(p=0.64)$. Additionally, the susceptibility of both iPSC lines to stimulated NKG2D $\%$ NKs cell was not significantly lower than to stimulated NK cells (iPSC 129/Sv: $p=0.1$; iPSC C57BL/6: $p=0.23$ ), but still not on the wildtype level. This shows that a stimulation with IL-2 increases the killing of stem cell lines by NKG2D-deficient NK cells and in single cases could almost compensate the NKG2D deficiency. 


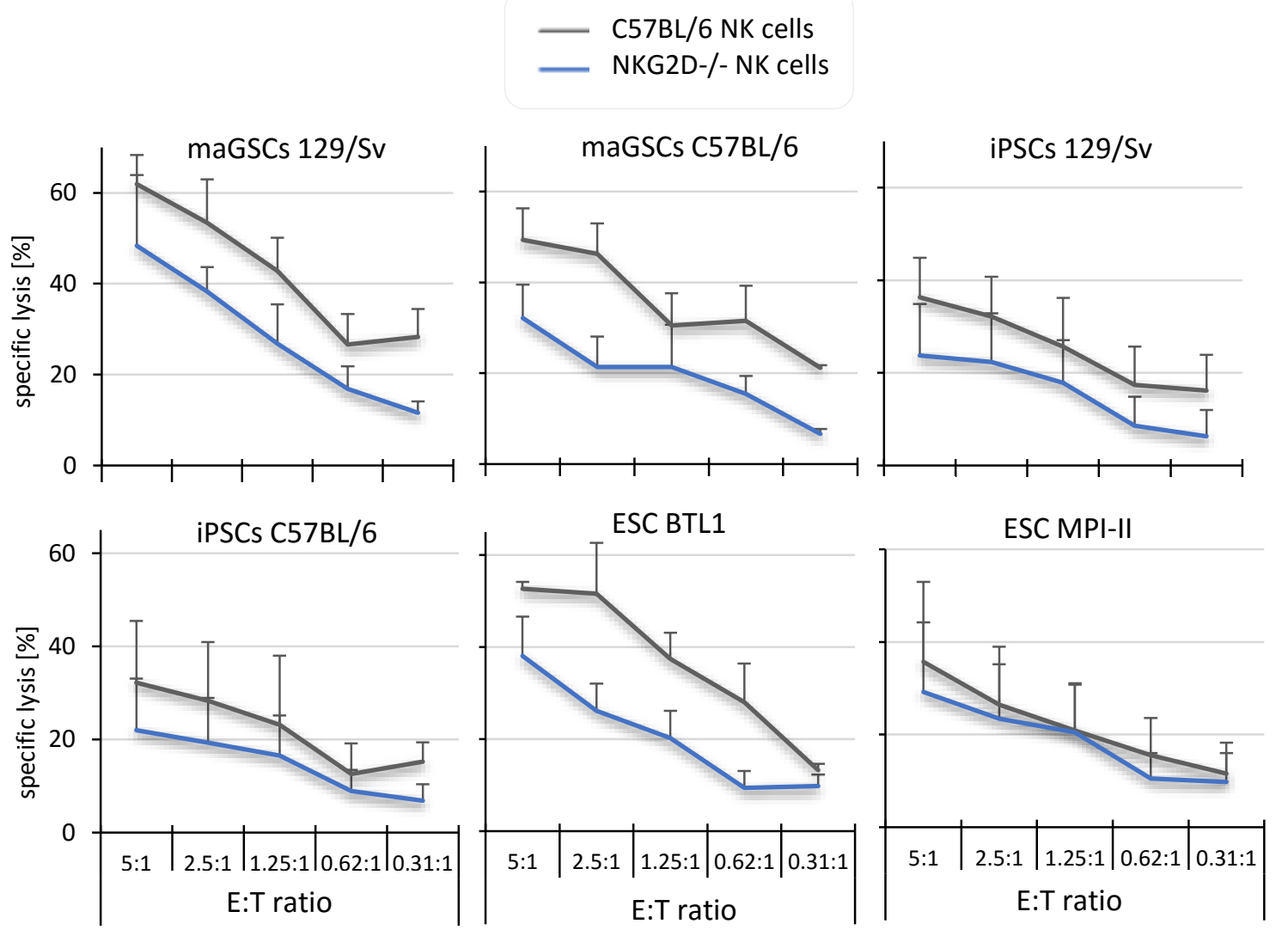

Figure 57 Killing of various stem cells lines by in vitro IL-2 stimulated wildtype C57BL/6 and NKG2Ddeficient NK cells.

Shown is the mean specific lysis + SEM; maGSCs 129/Sv $(n=3)$, maGSCs C57BL/6: $(n=3)$, iPSCs 129/Sv: $(n=$ 3), iPSC C57BL/6: $(n=3)$, ESC BTL1: $(n=3)$, ESC MPI-II: $(n=3)$.

\subsubsection{Expression of ligands of the NKG2D receptor on murine stem cells}

To determine the expression pattern of NKG2D ligands on the target cells flow cytometry analysis was performed using antibodies against RAE1, MULT1 and H60. All of the stem cell lines expressed these NKG2D ligands on their surface, but to a variable extent in number (cell positive for the ligands) and intensity (MFI) (Figure 58). The most abundant NKG2D receptor ligands was RAE1 which was detected in $40.2 \%$ (iPSC C57BL/6) to $76.0 \%$ (ESC BTL1) of the cells, except on maGSC C57BL/6, for which only $6.4 \%$ of the cells were positive. RAE1 expression analysis showed also the highest MFI values in the experiments, showing values from 4.9 (iPSC 129/Sv) up to 42.6 (maGSC 129/Sv). H60 was expressed on $2.7 \%$ (maGSC C57BL/6) to $46.8 \%$ (ESC BTL1) of the stem cells with similar MFI values of 1.4 (ESC MPI-II) to 3.4 (maGSC C57BL/6). The cell surface expression of MULT1 was the lowest of the NKG2D ligands, ranging from $2.8 \%$ (maGSC C57BL/6) to $10.0 \%$ (ESC-BTL1) with small MFI values of 0.1 (ESC MPI-II) to 3.1 (maGSC C57BL/6). YAC-1 was always included as NK cell target cell line and showed a 
high expression of all NKG2D ligands with $87.5 \%$ RAE1 ${ }^{+} ; 28.9 \% \mathrm{MULT1}^{+}, 49.6 \% \mathrm{H}^{+} 0^{+}$cells. Therefore, NKG2D expression on stem cell lines occasionally almost equals the expression on the control NK cell sensitive cell line YAC-1, e.g. RAE1 expression on ESC BTL1 or H60 expression on iPSC 129/Sv and ESC BTL1.
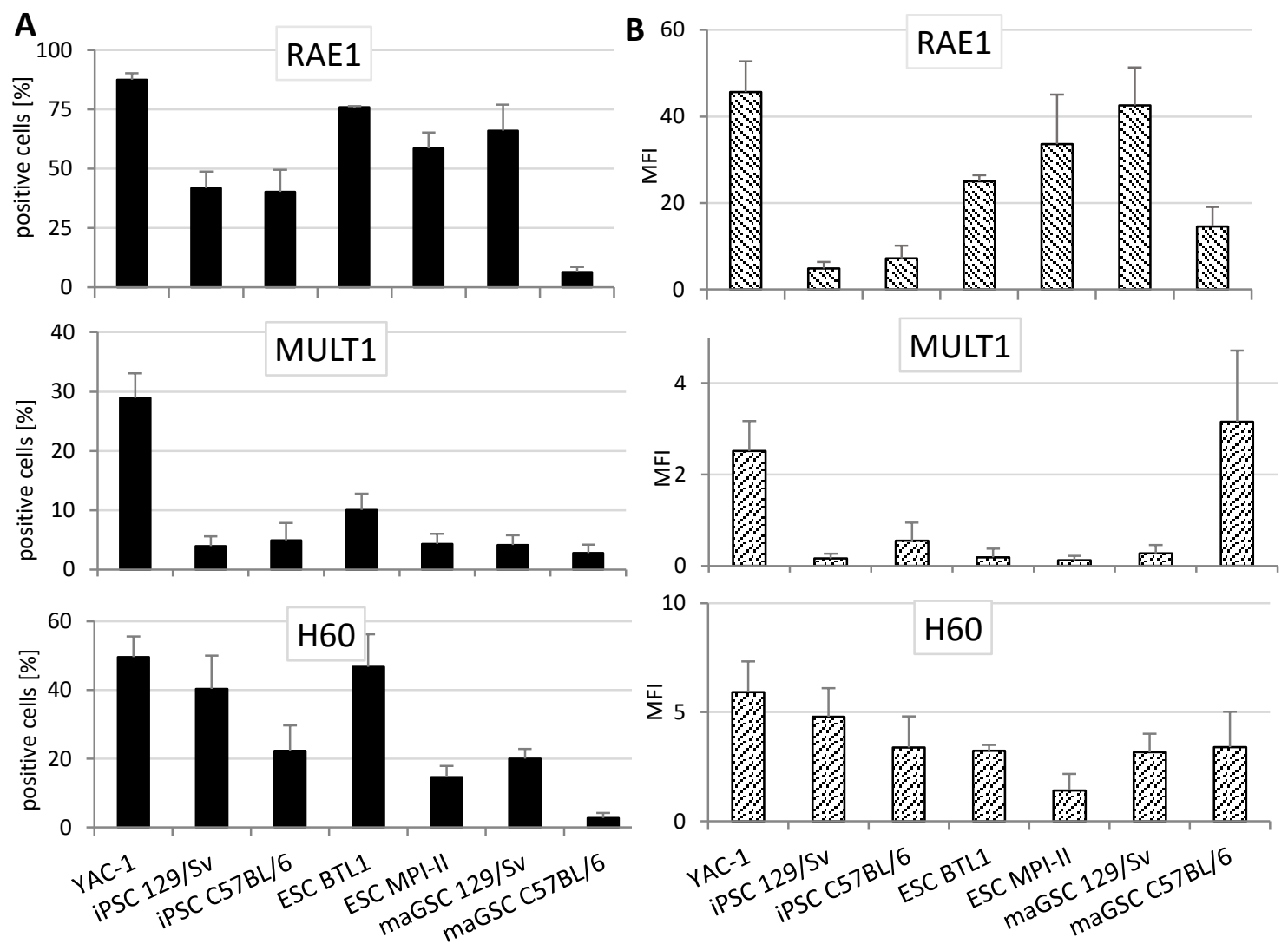

Figure 58 Expression of NKG2D ligands on stem cell lines and control (YAC-1) cell.

(A) Mean percentage of NKG2D ligand positive cells + SEM (B) Mean MFI (mean fluorescence intensity) of ligands expression on the cells + SEM; YAC-1 $(n=13)$, iPSC 129/Sv $(n=5)$, iPSC C57BL/6 $(n=8)$, ESC BTL1 $(n=$ 3), ESC MPI-II ( $n=7)$, maGSC 129/Sv ( $n=7)$, maGSC C57BL/6 $(n=7)$.

\subsubsection{Summary}

It was shown that the analyzed PSCs were largely resistant to killing by naïve NKG2D-deficient NK cells. In contrast, wildtype NK cells killed all targets with low or intermediate efficacy. The additional stimulation of wildtype and NKG2D-deficient NK cells by IL-2 increased the killing of all PSCs. Nevertheless, the NKG2D-deficiency was not fully compensated and wildtype NK cells were still more efficient. Furthermore, it was demonstrated that all PSC lines expressed NKG2D ligands although the expression pattern of RAE-1, MULT-1 and H60 molecules was variable. 


\section{Discussion}

\subsection{Involvement of autoimmune responses on the pathogenesis of load-induced heart failure}

Previous results of our group have indicated that autoimmune reactions against OVA can occur in cMy-mOVA mice after TAC. These mice express OVA exclusively on the surface of cardiomyocytes and thus enable the investigation of immune responses against this specific cardiac antigen. The mice were generated and first analyzed by Grabie et al., who showed that the mice display tolerance to OVA. Our previous results indicated that OVA-specific CTL responses after TAC were weak and stronger reactions not frequent in young and otherwise healthy mice. To examine whether autoimmunity is a more frequent outcome after TAC operations in animals at higher risk to develop autoimmunity, transgenic T cells with specificity for OVA were transferred into the cMy-mOVA mice. Moreover, double-transgenic mice were analyzed, which expressed OVA on their cardiomyocytes and an OVAspecific TCR on the majority of their $\mathrm{CD}^{+} \mathrm{T}$ cells. It was further investigated, whether autoreactive T cells, either endogenous or transferred, were sufficient to alter the cardiac remodeling after TAC operation or even accelerate the progression to HF.

\subsubsection{Increased leukocyte infiltration into the myocard after TAC}

Load-induced HF in C57BL/6 mice achieved by TAC surgery was accompanied with an increased leukocyte infiltration into the heart as analyzed by IHC (Toischer et al., 2010). Further analysis of this afterload model in our laboratory and by others revealed that TAC operations lead to infiltration of professional APCs and T cells into the myocardium (Laroumanie et al., 2014; Sasse et al., unpublished data). This was shown in our group for several time points from 1 day to 10 weeks post surgery by flow cytometric analysis. In this thesis, the infiltration of lymphocytes into the myocard was monitored at the experimental end point latest 10 weeks after surgery by IHC analysis of paraffin sections. At this time point a higher number of $\mathrm{CD}^{+}, \mathrm{CD}^{+}$, and $\mathrm{CD} 45 \mathrm{R}^{+}$cell were found after TAC than after sham operation for cMy-mOVA mice, cMy-mOVA mice which received either CD8 ${ }^{+} \mathrm{OT}-\mathrm{I}$ T cells or a mixture of $\mathrm{CD}^{+} \mathrm{OT}-\mathrm{I}$ and $\mathrm{CD} 4^{+} \mathrm{OT}-\mathrm{II} \mathrm{T}$ cells, and for cMy-mOVA/OT-II mice. The myocardia of animals that received OT-I T cells showed even higher infiltration of $\mathrm{CD}^{+}$but also $\mathrm{CD} 4^{+} \mathrm{T}$ lymphocytes than cMymOVA or cMy-mOVA/OT-II animals, indicating the recruitment of further lymphocytes besides the TCR-transgenic $\mathrm{CD}^{+} \mathrm{T}$ cells into the myocardium. The examination of the leukocyte composition in the myocardium via flow cytometry showed that after TAC operation, proportions of lymphocyte 
populations including B cells $(p=0.01)$, T cells, and NK cells were slightly increased compared to shamoperated animals. The general distribution of leukocyte populations in the heart resembled proportions of myeloid and lymphocyte cell proportions reported in a previous study (Bönner et al., 2012). This study demonstrated that $2.3 \times 10^{3}$ resident leukocytes /mg tissue were present in the unstressed heart of C57BL/6 mice, including a large proportion of APCs as sentinel cells of the myocardium (Bönner et al., 2012). These residential APCs in the heart might also play an important role in the presentation of autoantigens to T lymphocytes.

\subsubsection{Controversial influence of endothelial surface adhesion molecules on immune cell infiltration}

Moreover, we have investigated whether the increased infiltration of lymphocytes after TAC correlates with altered surface expression of adhesion molecules on residential cells. It was previously reported that endothelial cells show an activation in chronic HF in terms of expression of adhesion molecules and chemokines (Colombo et al., 2005; Tousoulis et al., 2005). Thus, endothelial cells might be essential for inflammation in $\mathrm{HF}$ by facilitating transendothelial migration of leukocytes. In humans and mice with $\mathrm{HF}$, it was reported that $\mathrm{T}$ cells showed a higher affinity towards the endothelium than controls (Nevers et al., 2015). Therefore, we examined whether an upregulation of the adhesion molecules VCAM-1 and ICAM-1 on endothelial cells in the heart occurs after TAC. However, no differences in the VCAM-1 and ICAM-1 surface expression in the myocard between sham- and TACoperated animals were found. This is in contrast to a study showing an upregulated protein expression of ICAM-1 on endothelial cells of left ventricular intramyocardial vessels after TAC (Nevers et al., 2015. Another recent study reported that ICAM-1-deficient mice displayed decreased T-cell and proinflammatory monocyte infiltration into the left ventricle after TAC surgery compared to controls, demonstrating the important role of ICAM-1 for immune cell infiltration (Salvador et al., 2016).

\subsubsection{Activation of adaptive immune responses towards cardiac antigens}

After the increase of lymphocytes infiltrating the myocardium in response to TAC operation and additional challenge by autoreactive T cells was demonstrated, it had to be clarified whether the OVAspecific lymphocytes were activated. A first hint of $C D 8^{+} T$ cell activation was found after transfer of CFSE-stained T cells. In the blood analysis at day 6 post transfer, a reduced number of CFSE-stained 
cells was found in animals, which underwent TAC surgery compared to sham-operated mice. However, this could indicate proliferation but also loss of cells or recruitment to other organs such as the heart. In cytotoxicity assays, the activation of OVA-specific CTLs was further proven in TAC- and shamoperated animals. In TAC-operated cMy-mOVA mice that did not receive an adoptive transfer of T cells, a significant but low activation of CTLs against OVA was detected, albeit single animals showed specific lysis rate of RMA OVA targets up to 15 \% (Röhrborn et al., unpublished data). In this thesis, the specific lysis rate of OVA-expressing target cells was higher for the animals that had received OT-I T cells. It was even further enhanced when OT-I and OT-II T cells were transferred, indicating an additional effect of OVA-specific CD4 ${ }^{+} \mathrm{T}$ cells on the activation of OVA-specific CTLs. However, this activation of T cells in cMy-mOVA mice that received OVA-specific T cells was largely independent of the type of surgery.

In C57BL/6 mice, which underwent TAC surgery, it was previously shown that more $\mathrm{CD} 4^{+}$and $\mathrm{CD} 8^{+}$ T cells expressed activation markers such as CD25 and CD69 (Sasse et al., unpublished data). CD4 ${ }^{+}$cells of cMy-mOVA/OT-II mice also expressed more CD25 after TAC than sham surgery. The expression level of $\mathrm{CD} 69$ on $\mathrm{CD}^{+} \mathrm{T}$ cells was not affected. Laroumanie et al. showed that $\mathrm{CD} 4^{+} \mathrm{CD} 44^{\text {high }}$ effector $\mathrm{T}$ cells numbers, in contrast to $\mathrm{CD} 8^{+} \mathrm{CD} 44^{\text {high }} \mathrm{T}$ cells were increased after TAC. These findings indicate a contribution of activated $\mathrm{CD} 4^{+}$cells on ongoing immune responses in the failing heart.

Examination of the cytokine profile of T helper cells in cMy-mOVA/OT-II mice after TAC operation revealed a polarization mainly towards an inflammatory $T h_{1}$ and $T h_{17}$ response, showing an increased proportion of $\mathrm{CD}^{+} \mathrm{T}$ cells positive for IL-6, IL-17A, IFNY and TNF $\alpha$. This was also previously found in C57BL/6 mice, in which more CD4 ${ }^{+}$cells expressing IFNY and IL-17A were present after TAC operation (Sasse et al., unpublished data). Nevers et al. (2015) reported an upregulation of TNF $\alpha$, IL-1 $\beta$, IL-6 and IFN $\gamma$ after TAC operation compared to controls. A further study confirmed a significant increase of $\mathrm{Th}_{1}$ cytokines, and a decrease of $\mathrm{Th}_{2}$ type cytokines in TAC-, compared to sham-operated animals (Laroumanie et al., 2014). While the $\mathrm{Th}_{1}$ response is triggering macrophage activation and cellmediated immunity, the $\mathrm{Th}_{17}$ response is involved in several autoimmune diseases in mice and human, including murine models of MS (EAE) (Komiyama et al., 2006), rheumatoid arthritis (Yen et al., 2006), psoriasis (Li et al., 2004a) and colitis (Littman and Rudensky, 2010). An upregulation ICAM-1 and the increased T cell infiltration after TAC was recently shown to be induced by cardiac cytokines IL-1 $\beta$ and IL-6 (Salvador et al., 2016). 
Generally, due to the detection of autoantibodies in patients and animals models of various cardiac diseases (Kaya et al., 2012), it was further analyzed whether antibodies were induced by increased afterload in cMy-mOVA mice. Analysis of these animals showed that no OVA-specific IgG autoantibodies were induced after TAC (Röhrborn et al., unpublished data). Challenging the mice with additional T cells against OVA in this thesis showed that neither in cMy-mOVA mice that obtained OVA-specific T cells by adoptive transfer, nor in cMy-mOVA/OT-II mice antibody generation against OVA was induced.

\subsubsection{The role of adaptive immune responses in the progression to heart failure}

Increased numbers of infiltrating lymphocytes suggested an involvement of adaptive immune responses in the progression to HF in the murine afterload model of TAC (Yndestad et al., 2006) as well as in humans (Yndestad et al., 2003). When wildtype and immunodeficient RAG2 ${ }^{-1} \mathrm{Yc}^{-/-}$mice were compared, it was found that lymphocytes promote cardiac hypertrophy in response to an increased afterload but are not required for the progression to HF (Sasse et al., unpublished data). However, analysis of $\mathrm{RAG}^{-/}$mice in another study revealed that the transition from hypertrophy to HF was attenuated at 6 weeks after TAC operation compared to immunocompetent animals (Laroumanie et al., 2014). This effect disappeared with reconstitution of $T$ cells into the immunodeficient mice (Laroumanie et al., 2014), inferring an effect of T cells on cardiac function. Generally, an increased afterload in C57BL/6 mice reduced heart function as measured by FAS and EF compared to control mice (Patten and Hall-Porter, 2009; Sasse et al., unpublished data; Toischer et al., 2010). These effect were not observed after TAC in mice deficient for TCR $\alpha$. These animals did not displayed hypertrophy or impaired cardiac function at 4 weeks after TAC surgery (Nevers et al., 2015), indicating an important role of T lymphocytes on the deterioration towards HF. Additionally, cardiac hypertrophy and fibrosis of the myocardium were not detectable in those mice, further suggesting T cells as regulators of these processes. The pharmacological depletion of T cells in the onset of HF by administration of an antiCD3 antibody into TAC-operated C57BL/6 mice led to improved cardiac function although mice displayed cardiac hypertrophy (Nevers et al., 2015). Laroumanie et al. (2014) examined the importance of $\mathrm{CD}^{+}$and $\mathrm{CD} 8^{+} \mathrm{T}$ lymphocytes on the adverse remodeling in $\mathrm{HF}$ by using either CD4deficient (MHC- $\left.-\mathrm{II}^{-/-}\right)$or $\mathrm{CD} 8$-deficient $\left(\mathrm{CD}^{-/}\right)$mice. In contrast to $\mathrm{CD} 8^{-/-}$mice which developed cardiac failure, CD4-deficient mice showed attenuated cardiac remodeling and preserved cardiac function at 6 weeks after TAC surgery compared to controls. Notably, OT-II mice, with transgenic CD4+ T cells specific for OVA, failed to develop HF and adverse remodeling after TAC (Laroumanie et al., 2014). 
These findings indicate an antigen-specific mechanism of $\mathrm{CD}^{+} \mathrm{T}$ cells that affects the progression to $\mathrm{HF}$, which was further investigated in the cMy-mOVA model.

\subsubsection{Increased numbers of autoreactive $T$ cells accelerate progression of load-induced heart failure}

Since various autoantibodies against cardiac antigens were found in patients with $\mathrm{HF}$ and other cardiac disease studies, autoimmunity was suggested to be associated with the progression to HF (Kaya et al., 2012). Röhrborn et al. analyzed whether the expression of OVA as cardiac antigen accelerates the progression to HF after TAC operation in cMy-mOVA mice. However, heart function was in general not altered compared to C57BL/6 wildtype mice. Moreover, the additional transfer of autoreactive T cells against OVA into cMy-mOVA mice was not sufficient to affect fibrosis, heart weight or hypertrophy neither after OT-I nor after OT-I and OT-II T cell transfer as shown in this thesis. Higher numbers of autoreactive $\mathrm{CD}^{+} \mathrm{T}$ cells in cMy-mOVA/OT-II mice accelerated progression to HF after TAC operation compared to cMy-mOVA mice. Unfortunately, the breeding of cMy-mOVA/OT-I mice failed due to early mortality of mice after birth (day 1 to 5). In conclusion, these findings demonstrate that a pathological increase of cardiac afterload can be sufficient to break the immunological tolerance of $\mathrm{CD}^{+}$and $\mathrm{CD} 8^{+} \mathrm{T}$ cells, even in young and healthy mice. In a model for virus-induced myocarditis, it was found that inoculation of an OVA-expressing vesicular stomatitis virus into cMy-mOVA mice only affected heart function after the animals additionally received adoptive transfer of naïve $\mathrm{CD} 8^{+} \mathrm{T}$ cells of OT-I mice (Grabie et al., 2003), indicating that immunological tolerance can be broken by virus infection if enough autoreactive CTLs are present.

The role of $\mathrm{CD}^{+}$cells was previously indicated to be important for the deterioration of cardiac function after TAC in C57BL/6 mice (Laroumanie et al., 2014), although our group could not prove their necessity for those processes (Sasse et al., unpublished data). In this thesis it was shown that high numbers of $\mathrm{CD}^{+} \mathrm{T}$ cells with specificity for a cardiac antigen are sufficient to induce autoimmune reactions after TAC and accelerate the progression to HF. Previous findings indicated an involvement of antibodies in immune responses responsible for the progression to HF due to detection of cardiac autoantibodies (Kaya et al., 2012). However, this thesis proved that heart function was affected by autoreactive $\mathrm{CD}^{+} \mathrm{T}$ cells independent of antibody production against cardiac antigens. 


\subsubsection{Immune mechanisms contributing to heart failure}

Generally, in response to tissue injury in the heart, resulting from pathogens or environmental injury such as hemodynamic overloading or ischemia, innate immune responses become activated. Cardiac innate immune responses are initiated by the detection of damage associated molecular patterns (DAMPs) or pathogen associated molecular patterns (PAMPs) by pattern recognition receptors (PRRs), which not only detect infiltrating pathogens, e.g. bacteria but were also shown to recognize molecular patterns of endogenous material released after heart injury by damaged or dying cells (Mann, 2015). Subsequently, neutrophils and monocytes in the damaged tissue area become activated through engagement of extracellular or intracellular PRRs, leading to short term adaptation of the heart to the abnormal conditions. In addition to many resident tissue macrophages in the heart, which were established during embryonic development and contribute to cardiac recovery, the infiltration of bone marrow-derived neutrophils and monocytes lead to a pro-inflammatory stress response within the myocardium. This has been shown to contribute to further tissue damage, progressive fibrosis and adverse cardiac remodeling (Epelman et al., 2015; Lavine et al., 2014). It is assumed that the myocardium adapts to environmental stress by synthesizing a variety of cytoprotective factors such as adenosine and nitric oxide (Bolli et al., 1997; Downey et al., 1993). It responds to various endogenous stress-induced factors including basic fibroblast growth factor (bFGF), TGF $\beta$, vascular endothelial growth factor (VEGF) and angiotensin II (Eghbali, 1989; Hammond et al., 1984; Li et al., 1997; Sadoshima et al., 1993; Weiner and Swain, 1989). This limits cell injury by upregulation of cytoprotective factors and facilitate subsequent tissue repair. If cardiac homeostasis cannot be restored, overexpression of these factors can lead to a chronic low grad inflammation, which contributes to further disease progression and infiltration of further immune cells. An increased afterload was shown to lead to a significant up-regulation of Toll-like receptor genes, such as TIr2, apoptosis-related genes such as Casp3, genes encoding chemokines attracting myeloid cells including Ccl9, genes for adhesion molecules (Icam-1, Vcam-1, E-selectin), and chemokine receptor genes expressed on myeloid cells, such as Ccr1 as examined in hearts of C57BL/6 mice 1 week (Sasse et al., unpublished data) or 4 weeks after TAC (Nevers et al., 2015). Additionally, stress-inducible ligands including Rae1, Ulbp1 and $\mathrm{H} 60$ of the murine NK receptor NKG2D, which functions as costimulatory molecule on T cells were found to be significantly upregulated 1 week after TAC surgery (Sasse et al., unpublished data). Usually, these mouse ligands and their human counterparts (MICA, MICB, ULBP1-6), are constitutively expressed on only few cell types but become inducible by proteotoxic and genotoxic stress in most cells and have been implicated in immune responses to infections, tumors 
but also in many autoimmune diseases (Ogasawara and Lanier, 2005; Ogasawara et al., 2004; Salih et al., 2003). The gene encoding NKG2D itself, KIrk1, was also significantly up-regulated at this time point suggesting the presence of cells, which can respond to the NKG2D ligands (Sasse et al., unpublished data). Due to the early upregulation of these ligands in the myocardium after TAC surgery, nonprofessional APCs could co-stimulate and thereby activate naïve T cells, which were found to infiltrate the myocardium. Thus, $T$ cells with specificity for cardiac autoantigens might become activated also by non-professional APCs including cardiomyocytes. Additionally, professional APCs, i.e. DCs, could take up antigens released from damaged cells in the myocardium, maturate in response to DAMPs and proinflammatory cytokines, and activate autoreactive T cells in regional lymph nodes that escaped central tolerance. Notably, it was found that not all cardiac antigens are expressed by thymic medullary epithelial cells as shown for $\alpha-\mathrm{MHC}$, revealing that $\alpha-\mathrm{MHC}$ reactive $\mathrm{CD} 4^{+} \mathrm{T}$ cells were even found in the blood of healthy patients (Lv et al., 2011). After activation of T lymphocytes, autoreactive cells either directly affect the myocardium, as e.g. CTLs do, or enhance the inflammatory function of other cells e.g. by helping B cells produce pathogenic antibodies as shown for $\mathrm{T}$ helper cells. Additionally, the polarization of $\mathrm{CD}^{+} \mathrm{T}$ cells, into $\mathrm{Th}_{1}$ and $\mathrm{Th}_{17}$ cells, as shown in cMy-mOVA/OT-II mice seems to exert adverse effects on the myocardium. Significantly more $\mathrm{CD}^{+} \mathrm{T}$ cells expressing

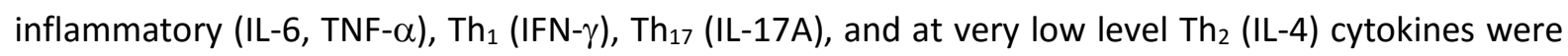
present after TAC surgery compared to controls. IFN- $\gamma$, which is produced by $\mathrm{CD} 4^{+} \mathrm{Th}_{1}, \mathrm{CD} 8^{+} \mathrm{CTL}$, NK and NKT cells, is known to have multiple proinflammatory effects (Lichtman, 2013). However, it also exhibits anti-inflammatory effects including feedback inhibition of effector $T$ cells that produce the cytokine, thereby limiting $T$ cell activation and $T$ cell mediated inflammatory damage to the myocardium (Lichtman, 2013). Moreover, IL-6 seems to play a critical role in the pathogenesis of LV hypertrophy in response to pressure overload since genetic deletion led to alleviation of hypertrophy and cardiac dysfunction (Zhao et al., 2016). Th ${ }_{17}$ cells produce IL-17, a cytokine associated with neutrophilic inflammation, that was shown to affect cardiac fibroblasts, thereby promoting fibrosis, but not the severity of inflammation in autoimmune myocarditis (Baldeviano et al., 2010). Moreover, it has been shown that a blockade of IL-17A improves the LV function of the heart and lowers cardiomyocyte apoptosis and neutrophil recruitment (Liao et al., 2012). The fact that infiltrating T cells have influence on cardiac remodeling of the myocardium was additionally proven in a mouse model of T cell deficiency $\left(\mathrm{TCR}^{-/}\right)$since these mice displayed reduced fibrosis and hypertrophy, improved cardiac function and survival after TAC operation compared to wildtype mice (Nevers et al., 2015). 
In conclusion, inflammatory $\mathrm{CD}^{+}{ }^{+} \mathrm{T}$ cells and their cytokine products are involved in the progression to HF, leading to an increased damage of the myocardium.

\subsubsection{Summary and future perspectives}

These data show that CTLs as well as $\mathrm{CD} 4^{+} \mathrm{T}$ cells specific for OVA as cardiac antigen can become activated in a model of increased afterload and promote the progression from hypertrophy to HF. Interestingly, $\mathrm{T}$ helper cells seem to deteriorate heart function by a mechanism independent of antibody induction, since no OVA-specific antibodies were found neither in cMy-mOVA/OT-II nor in cMy-mOVA mice which received adoptive transfer of $\mathrm{CD}^{+}$and $\mathrm{CD} 8^{+} \mathrm{T}$ cells. Such direct effects on heart function might be of interest if those autoreactive T cells exist in subgroups of patients with HF either alone or in combination with cardiac autoantibodies, since autoimmune processes can be targeted by established or newly developed therapeutics. Thus, it could be possible to decrease or slow the risk of transition into manifest $\mathrm{HF}$ in those patients and prevent long term immunosuppression if the activation of these autoreactive $T$ cells could be prevented by short-term intervention in time periods of increased risk, e.g., after acute cardiac ischemia.

\subsection{Stem cell immunogenicity}

Stem cells are important tools for future regenerative medicine since they can be differentiated to various cells types and therefore replacing or promoting damaged or diseased tissue. However, it is necessary to evaluate the immunogenicity of transplanted material to reduce the risk of graft rejection. The role of the highly polymorphic MHC was previously studied, whereas miHC antigens were so far mostly disregarded. However, clarification of the immunogenicity of those antigens is critical since they cannot be matched in allogenic transplantations, which are expected to be clinically most relevant. Therefore, OVA-expressing stem cells, i.e. iPSCs and ESCs, were analyzed regarding their engraftment and their effects on effector cell activation in otherwise syngeneic recipients. Additionally, expression of immune-evading or immunogenicity-causing genes in stem cells was analyzed to better estimate the immunogenic potential of pluripotent stem cells. 


\subsubsection{Influence of minor histocompatibility antigen expression on stem cell immunity}

The expression of other antigens besides $M H C$ and $A B O$ antigens were reported to influence the outcome of transplantation, i.e. transplantations between MHC-matched individuals led to the development of GVHD or resulted in T cell-mediated graft failure (Ferrara et al., 2009; Opelz and Collaborative Transplant Study, 2005). These immune reactions are likely elicited by the expression of variant miHC antigens between donor and recipient, leading to activation of $\mathrm{CD} 4^{+}$and $\mathrm{CD} 8^{+} \mathrm{T}$ cell alloresponses. To study the effects of miHC antigen expression for transplantations of stem cellderived grafts, several murine stem cell lines were transfected with an OVA-eGFP fusion construct to express the model antigen OVA (Monecke, 2013). The analysis of OVA-expressing stem cell in vitro revealed that the antigen processing and consequently antigen presentation of murine stem cells was impaired (Monecke, 2013). The antigen processing and presentation via the MHC class I pathway leads to the cell surface expression of MHC class I molecules, which confers susceptibility of cells to the cytotoxicity of CTLs. As previously reported, murine PSCs lack surface expression of MHC class I molecules, which is also not inducible by IFNY (Abdullah et al., 2007; Monecke, 2013; Nussbaum et al., 2007; Tian et al., 1997). The stem cell lines transfected with OVA-eGFP were not killed by OVA-specific CTLs in vitro analyzed in cytotoxicity assays (Monecke, 2013). However, the supplement of processed OVA peptide SIINFEKL increased the lysis rate of all tested stem cells, including iPSCs, ESCs and maGSCs, demonstrating that PSCs were not generally protected against CTL cytotoxicity. In co-culture experiments with OVA-specific T cells, it was demonstrated that PSCs were able to actively suppress T cell activation in vitro, mediated by cell-cell contact of PSCs and T cells (Monecke, 2013). In addition to data describing immune-escape or tolerance-inducing mechanisms of stem cells, e.g. by expression of Arg1 and IDO, these findings suggest that PSC are at least in vitro resistant against immune responses. Nevertheless, T cell-mediated immune responses against murine PSCs were monitored in vivo (Boyd and Wood, 2009; Dressel et al., 2009; Robertson et al., 2007; Wu et al., 2008). To further characterize the effect of miHC antigens expressed by stem cells or stem cell-derived grafts on engraftment and stem cell immunogenicity, OVA-expressing stem cells were analyzed in vivo compared to their wildtype counterparts in this thesis. It was hypothesized that adaptive immune reactions against OVA as a single miHC antigen in otherwise syngeneic hosts might affect transplantations of stem cells and stem cell-derived grafts in vivo. For this, OVA-expressing ESCs and iPSCs and wildtype stem cells were injected into otherwise syngeneic mice to examine their engraftment in teratoma formation assays and the potential induction of OVA-specific T and/or B lymphocytes. 


\subsubsection{Expression of OVA as miHC antigen affects engraftment of stem cells in immunocompetent syngeneic hosts}

To exclude clonal abnormalities due to OVA expression, OVA-expressing and wildtype stem cells were inoculated into immunodeficient mice to monitor teratoma growth and exclude potentially aberrant cell clones. The expression of OVA on iPSCs and ESCs showed no general effects on teratoma formation compared to wildtype stem cells. To examine whether OVA-specific T cells impair the engraftment of OVA-expressing PSCs, TCR-transgenic T cells, i.e. $\mathrm{CD} 4^{+}$and/or $\mathrm{CD} 8^{+} \mathrm{T}$ cells, specific for OVA, were transferred into the immunodeficient animals after stem cell inoculation. However, the presence of the OVA-specific T cells did not impair engraftment of OVA-expressing stem cells. In contrast, inoculation of OVA-expressing stem cells into immunocompetent but otherwise syngeneic animals revealed differences in the engraftment, i.e. tumor formation rate, compared to inoculated wildtype stem cells. Animals which were inoculated with OVA-expressing iPSCs or ESCs displayed a significant reduced tumor formation rate, suggesting OVA-specific immune responses towards the transplanted cells. Generally, tumors derived from OVA-expressing stem cells were significantly smaller and lighter compared to teratomas of wildtype stem cells and to those formed in immunodeficient mice, indicating an influence of immune cells on the formation of teratomas. The analysis whether Y-chromosome derived (HY) antigens might additionally influence the outcome of transplantation, tumor formation of male stem cells in female host was monitored. Noticeably, the expression of those antigens seem to have an additional effect on tumor formation since tumor rate was markedly reduced in female mice ( $8 \%$ ), compared to male hosts (46\%) but only for tumors derived from OVA-expressing stem cells. This indicates an enhancing effect of $\mathrm{HY}$ antigens on the rejection process of OVA-expressing stem cells. These data are in accord with a previous small scale study in our group (Monecke, 2013). Analysis of mouse embryogenesis revealed that HY antigens are already expressed at the eight cell stage of the embryo and might therefore affect stem cell immunogenicity (Krco and Goldberg, 1976). Infiltration of leukocytes was observed in all teratomas derived from wildtype and OVA-expressing stem cells. However, teratomas derived from OVAexpressing stem cells showed increased numbers of infiltrating leukocytes. The infiltration of leukocytes into teratomas derived from PSCs in syngeneic hosts was previously shown in several studies (Dressel et al., 2008; Kofidis et al., 2005).

Clonal differences in proliferation capacity and growth rate between cell clones resulted in slightly variable tumor formation rates, variable tumor size, weight and variations in the duration of experiments in immunodeficient mice. In immunocompetent mice, additional miHC antigen 
expression like HY antigens and antigen independent effector mechanisms as NK cell killing possibly contributed to smaller differences in tumor size and weight. Monitored distinctions between tumors derived from OVA-expressing stem cell clones might be explained by the variant expression of OVA by single cell clones and its reduction with differentiation. QPCR analysis revealed that the transgene expression was distinctly reduced in teratomas compared to its expression in PSCs. The loss of transgene expression over differentiation might happen due to epigenetic changes during the differentiation process, resulting in silencing of the genomic loci in which the OVA-eGFP transgene had integrated. To overcome this problem, the transgene construct was inserted into the Rosa26 locus, which was reported to be constitutively expressed with differentiation (Kisseberth et al., 1999). Investigations concerning the immunogenicity of those stem cells are still ongoing (Petrov et al., unpublished data). Transgene expression differed between iPSCs and ESCs but also displayed interclonal variations which affect the overall immunogenicity of each stem cell clone. An already low transgene expression in teratomas formed in immunodeficient mice can be explained by transgene loss due to differentiation. In contrast, a lower expression of OVA in immunocompetent than in immunodeficient animals was probably caused by immune effecter activation against OVA which eliminates OVA-expressing cells. Despite the loss of OVA expression with differentiation, it was demonstrated that inoculated OVA-expressing stem cells displayed a significantly reduced rate of engraftment in immunocompetent mice. This demonstrates that the expression of OVA was still sufficient to elicit an immune effector response.

\subsubsection{Expression of a miHC antigen led to OVA-specific cytotoxic T lymphocyte induction}

Already immunodeficient animals which were inoculated with OVA-expressing stem cells and additionally received transfer of OVA-specific T cells from OT-I and OT-II mice displayed altered immune responses compared to animals which received wildtype PSCs. Analysis of transferred T cells shortly after the transfer and after experimental ending revealed that $\mathrm{CD} 8^{+} \mathrm{T}$ cells became activated, showing proliferation in vivo and were able to directly kill OVA-expressing target cells when tested in vitro in cytotoxicity assays. Surprisingly, all OVA-expressing stem cell injections resulted in teratoma formation despite the proven activation of OVA-specific $T$ cells and their infiltration into the teratomas. However, an influence of these cells on teratoma growth was shown in animals that displayed rapid proliferating $\mathrm{CD} 8^{+} \mathrm{T}$ cells, which was associated with a prolonged survival of the hosts. Albeit, $T$ cells were not able to completely eliminate tumors because of either stem cell numbers 
outranging small numbers of transferred T cells or the loss of transgene expression in stem cells over differentiation. In syngeneic immunocompetent host, the effect of OVA expression in the inoculated stem cells was investigated in an immunologically fully functional environment. To determine the activation status against OVA as miHC antigen, splenocytes were in vitro restimulated after experimental ending (up to 90 days) and used as effector cells in cytotoxicity assays. Noticeable, only splenocytes of animals which received injections of OVA-expressing stem cells were able to efficiently kill the OVA-expressing RMA target cell line, in contrast to splenocytes of animals, which received wildtype stem cells. This indicates that OVA-specific CTLs arose in the animals that obtained injections of OVA-expressing stem cells. Additionally, this could be already shown in animals 1 week after stem cell inoculation, revealing an early activation of CTLs after stem cell injection. Surprisingly, even the injection of not fully pluripotent stem cells whose injection did not led to tumor formation in immunodeficient mice, led to an induction of OVA-specific CTLS in immunocompetent hosts. Consequently, the inoculation of OVA-expressing stem cells, which were not able to proliferate, differentiate or form teratomas in vivo, was sufficient to activate immune effector responses against the expressed antigen. PSCs did not express MHC class I molecules but these are required for peptide presentation to activate CTLs. It is known that with differentiation of PSCs towards embryoid bodies (EBs), MHC class I molecule expression is upregulated (Petrov et al., unpublished data; Suárez-Alvarez et al., 2010). Additionally, current investigations in our group showed that components for the antigen processing machinery are also increased with differentiation to EBs (Petrov et al., unpublished data), leading to an augmented potential to present peptides and to become targets of peptide-specific CTLs. OVA-specific CTLs must have exerted an effect on the growth of OVA-expressing PSCs after differentiation, since the PSCs themselves were not able to process and present this antigen. However, some teratomas might have escaped rejection by down-regulation of OVA expressions.

\subsubsection{Expression of a miHC antigen resulted in OVA-specific antibody generation and $\mathrm{CD} 4^{+} \mathrm{T}$ cell activation}

To clarify whether the expression of OVA in stem cells led to B cell activation and antibody production, sera of animals were examined in ELISA assays to determine the anti-OVA antibody titer in animals, which received either wildtype or OVA-expressing stem cells. At the early point of time 1 week after stem cell inoculation, no induction of anti-OVA antibodies were detected in the sera of animals. However, after experimental ending (up to 90 days), anti-OVA antibodies were found in animals, which received OVA-expressing stem cells, equaling OVA titers of previously OVA-immunized control 
animals. Strikingly, anti-OVA titers were significantly higher in animals, which received OVA-expressing stem cells and displayed teratoma formation in contrast to animals, which obtained the same stem cells but did not develop any tumors. This could be explained by the early activation of CTLS or NK cells in the host, leading to rapid rejection of the graft and elimination of the immunogenic antigen, i.e. OVA. In contrast, if those cells were not able to completely reject teratomas, OVA would be expressed in the forming tissue, enabling an increased immune response against this miHC antigen by $\mathrm{CD} 4^{+} \mathrm{T}$ and $B$ cells.

Whether $\mathrm{CD} 4^{+} \mathrm{T}$ cells specific for OVA were involved in the antibody generation of hosts was further determined by characterization of generated antibody isotype subclasses. Major proportion of OVA antibodies were of the $\lg G 1$ and $\lg G 2 \mathrm{~b}$ isotypes, indicating a class switch in OVA-specific $B$ cells which requires the interaction with antigen-specific $\mathrm{CD}^{+} \mathrm{T}$ cells to induce the class switch since the OVA protein is a thymus-dependent antigen.

Generally, the presumed time for humoral response after primary antibody exposure is up to 14 days (Ademokun and Dunn-Walters, 2010). Consequently, humoral response against OVA was not detectable early after transplantation but developed with tumor formation of inoculated stem cells, showing that expression of miHC antigens is sufficient to induce an antibody response of the host. At this, not only antigen-specific $B$ lymphocytes but also antigen-specific CD4 ${ }^{+} \mathrm{T}$ lymphocytes became activated in syngeneic hosts after inoculation of stem cells expressing OVA as single miHC antigen. Due to the absent expression of MHC class II molecules, PSC itself unlikely function as professional APCs for $\mathrm{CD}^{+} \mathrm{T}$ cell activation. However, with differentiation to epithelial cells which serve as atypical APCs since they express MHC class II molecules, $C D 4^{+} \mathrm{T}$ lymphocytes could be activated by direct allorecognition (Hershberg et al., 1998; Kreisel et al., 2002). More likely, OVA peptides derived from apoptotic PSCs or teratoma cells are taken up by host APCs and presented to CD4 ${ }^{+}$T lymphocytes via the indirect allorecognition pathway. Activated Thelper cells then further activate and enhance CTL and B lymphocyte effector function.

\subsubsection{NK cells supported killing of stem cells}

Due to the rather low expression of MHC class I molecules, which served as ligands for inhibitory NK cell receptors, stem cells can also become targets for NK cells. The susceptibility of PSCs to NK cell killing was previously shown for murine and human cells, demonstrating that besides xenogeneic and allogeneic also syngeneic NK cells were able to effectively kill stem cells (Dressel et al., 2008, 2010; 
Kruse, Hamann et al., 2015; Tian et al., 2006). Therefore, the contribution of NK cell killing in the rejection of inoculated stem cells was additionally examined by cytotoxicity assays in which a NK cellsensitive target cells line was used as target cell. The lysis of the target cell line by splenocytes obtained of animals that received OVA-expressing stem cells was distinctly higher compared to lysis by splenocytes of hosts, which were injected with wildtype stem cells. At this, a proinflammatory environment due to adaptive effector responses against OVA would increase the capability of NK cells to kill PSCs.

\subsubsection{Analysis of mechanisms potentially influencing the immunogenicity of stem cell and stem cell-derived grafts}

\subsubsection{Negligible expression of immunogenic genes in stem cells and their derivates}

Initially, Zhao et al. reported that iPSCs were associated with the overexpression of so-called immunogenicity-causing genes, affecting the outcome of transplantion, i.e. teratoma formation, in a mouse model (Zhao et al., 2011). Since then, several investigations concerning the potential expression and impact of these genes, Cyp3a11, Hormad1 and $\mathrm{Zg} 16$, on the immunogenicity of iPSC compared to ESCs were implemented. Murine studies showed that in contrast to the initial study, neither differentiated cells nor undifferentiated iPSCs displayed variant expression of those genes compared to ESCs and their derivates (Araki et al., 2013; Guha et al., 2013). These findings were confirmed in this thesis since expression of Zg16, Hormad1 and Cyp3a11 in PSCs and derived teratomas was detected only on a minimal level and did not show noticeable variations between stem cell types or differentiation status. In accordance with these findings, examination of human ESCs and iPSCs via $\mathrm{qPCR}$ and flow cytometric analysis demonstrated similar gene and protein expression of Zg16, Hormad1 and Cyp3a11 regardless of their differentiation status (Maynard et al., 2014). Nevertheless, further analysis of ZG16 and HORMAD1 in human stem cells and their differentiation

products evinced that HORMAD1 expression in hESCs and hiPSCs derivates was very low and heterogeneous, but ZG16 expression increased significantly on differentiation of hPSCs into clinically relevant cell derivates as hepatocytes, cardiomyocytes and oligodendrocytes (Awe et al., 2015). However, modified peripheral blood mononuclear cell (PBMC) co-culture assays in vitro revealed no acute immune response due to ZG16 expression, indicating no significant immunogenicity of this gene (Awe et al., 2015). 


\subsubsection{Expression of immune-mediating enzymes}

Immune privilege was previously reported for several stem cell types including ESCs and mesenchymal stem cells (MSCs) (Li et al., 2004b; Uccelli et al., 2008). It comprises cellular, molecular or anatomical mechanisms to prevent cells or tissue from immunological pursuit by the immune system (Figure 59). This includes passive forms of antigenic invisibility, e.g. achieved by the lack of antigen presentation, or active immune regulation to prevent antigen recognition. Evasion of antigen recognition due to immunological barriers, e.g. the blood brain barrier (BBB), can lead to immune activation if that isolation breaks down or is bypassed by primed immune cells, eventually causing autoimmunity. Therefore, most of the classical immune-privileged sites, e.g. the eye, exhibit additional active immune regulation, which locally suppress the adaptive immune response against a target antigen. ESCderived tissues as well as adult stem cells were reported to exhibit several of these features to evade immune recognition by the organism.

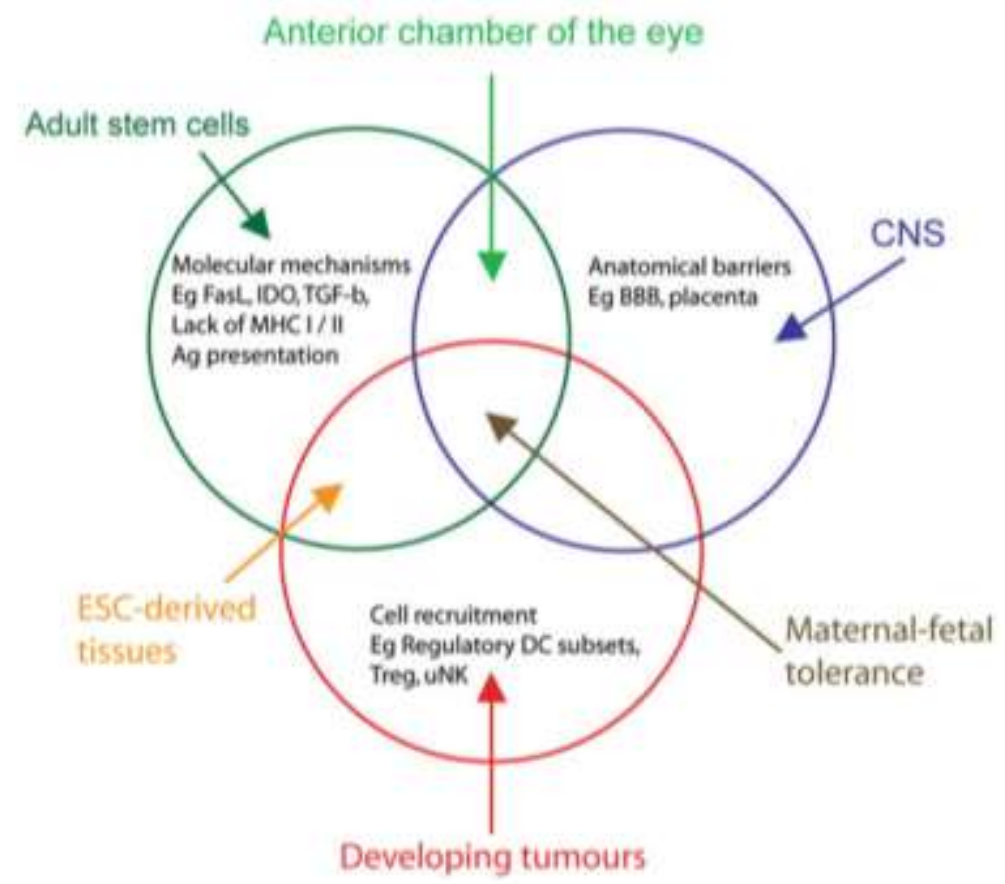

Figure 59 Strategies to achieve immune privilege, e.g. by cell recruitment, anatomical barriers or various molecular mechanisms.

Either immune-privileged sites possess mechanisms of active or passive immune evasion, or both simultaneously. uNK: uterine NK cells; BBB: blood brain barrier; ag: antigen. Picture by Zavazava, 2013

Despite their restricted differentiation potential, adult stem cells are required for the homeostasis and regeneration of various tissues in the adult organism. They are located in specialized niches, kept quiescent and uncompromised by autoimmune or inflammatory responses until their proliferation and differentiation is needed (Liu et al., 2009; Uccelli et al., 2008; Zavazava, 2013). It is postulated that these niches serve as a protective microenvironment besides the own immune-privilege of the adult 
stem cells. However, it was highly debated whether ESCs, derived from the ICM of the early blastocyst, exhibit similar immune escape properties. Additionally, the immune regulatory capabilities of other stem cell types were investigated since it is important for future regenerative medicine to achieve tolerance to transplanted grafts by the recipient.

A possibility to influence immune recognitions is the limitation of essential amino acids in a local environment. This can lead to interference of immune responses since lymphocytes require those fore rapid proliferation and effector function and are therefore especially sensitive to amino acid starvation (Calder, 2006; Chuang et al., 1990). Consequently, lowering the availability of essential amino acids in a tissue might lead to acquisition of immune privilege. It was previously shown that Indoleamine 2,3-dioxygenas (IDO), an intracellular enzyme that catabolized tryptophan, is not only an important factor for immune privilege during pregnancy but its expression was also found in DCs (Metz et al., 2007; Munn et al., 1998). Here, antigen presentation during tryptophan deprivation cause a polarization of $\mathrm{CD}^{+} \mathrm{T}$ cells towards the Treg phenotype (Fallarino et al., 2006). Additionally, the depletion of other essential amino acids, e.g. of arginine by Arginase 1, were found to modulate T cell responses (Bronte and Zanovello, 2005; Cobbold et al., 2009). It is assumed, that Ido expression on MSCs led to an immunosuppressive function in the human body (Su et al., 2014). In previous murine studies, only low amounts of Ido RNA and protein were found on ESCs and no contribution of IDO to immune suppressive activity of ESCs could be confirmed (Han et al., 2011; Monecke, 2013). In accordance with these studies, only very low RNA expression of Ido was discovered in PSCs in this thesis. After in vivo differentiation into teratomas, most tumors showed an increased level of Ido expression compared to PSCs but still lower than placenta tissue. However, in single animals, a tremendous amount of $I d o$, was expressed in teratomas derived from ES cells, outweighing the expression in the control. This indicates that undirected differentiation of stem cells can lead to an upregulation of Ido expression, which might influence the immunogenicity of the cells or tissue. In literature, this could be shown for several carcinomas, in which overexpression of Ido was associated with immune escape mechanisms of those tumors (Brandacher et al., 2006; Ozaki et al., 1988; Pan et al., 2008; Platten et al., 2012; Thaker et al., 2013). For Arg1 is was previously reported that its expression in tumor cell led to an ineffective anti-tumor immune response, but did not result in the complete inhibition of anti-tumor immune reactions (Korrer and Routes, 2014). However, other studies showed that the amino acid Arginine is important for tumor cells and suggested ARG1 as potential enzyme for anti-cancer therapy (Patil et al., 2016). In previous ES cell studies, it was demonstrated that a high expression of ARG1 in human ESC lines HES-1 and HES-2 led to suppression 
of T cell activity (Yachimovich-Cohen et al., 2010). However, in vitro studies of murine ESCs revealed that Arg1 was not involved in the inhibition of co-cultured T cells since its expression was not detectable via qPCR analysis (Monecke, 2013). However, in this thesis a low expression of Arg1 was discovered in IPSC and ESC clones, which increased with differentiation during teratoma formation. However, whether this influences the immunogenicity of stem cell-derived tissues needs to be clarified.

\subsubsection{MiHC antigen expression affects engraftment by inducing an antigen dependent immune response - Summary and Outlook}

We could prove in this thesis that the expression of miHC antigens in PSCs led to adaptive immune effector activation in vivo. Thus, the in vivo immunogenicity of PSCs is highly different to its previously reported in vitro non-immunogenicity. In vitro, PSCs exhibited rather immunosuppressive features. However, with transplantation, the risk of indirect antigen presentation increases due to protein release from damaged or apoptotic cells. Additionally, the upregulation of MHC class I, as caused by differentiation of PSCs and/or an inflammatory environment, increases the risk of peptide-specific CTL responses towards miHC antigens. Consequently, variant miHC antigens have to be considered as immunogenic in PSCs in allogeneic transplantation settings even when MHC molecules are matched.

MiHC antigens are generated by the intracellular degradation of polymorphic proteins which originate from polymorphisms in single nucleotide polymorphisms (SNPs), copy number variations (CNVs) or base-pair insertions or deletions (indels) (Hombrink et al., 2013). Since PSC-derived grafts are seen as new source for therapeutic transplantations, potential acquisition of neoantigens might occur due to incomplete or aberrant reprogramming of adult donor cells or the reprogramming itself, e.g. after integration of transcription factors into the genome (Takahashi and Yamanaka, 2006). Since the immune system has not encountered those antigens before, it is most likely that the tolerance towards these neoantigens is lacking. It was already reported that in more than $80 \%$ of analyzed healthy patients OCT4-specific T cells, mainly $\mathrm{CD}^{+}{ }^{+}$cells, were detected, indicating a feasible immune response against cells expressing this embryonic antigen like stem cells (Dhodapkar et al., 2010).

Currently described miHC antigens include antigens encoded e.g. by sex chromosomes, derived from the $\mathrm{Y}$ chromosome, known as HY antigens. HY antigens are expressed among others on human hematopoietic progenitor cells which were already early assumed to affect hematopoietic stem cell transplantations (HSCT) (Voogt et al., 1988). HY antigens play also a role in graft rejection of solid 
organs, in which HY-mismatching was shown to correlate with clinical outcome and acute graft rejection (Gratwohl et al., 2008; Tan et al., 2008). Moreover, several miHC antigens are encoded by autosomal genes, as HA1 - HA8, which are even found in mitochondrial DNA, named maternally transmitted antigens (MTA) (López-Larrea, 2012). It was shown for SCNT-derived ESCs that mismatched mitochondria affected graft survival, however the group did not clarify by which mechanisms (Deuse et al., 2015). The tissue distribution of known miHC antigens ranged from exclusive expression on hematopoietic cells, i.e. HA1, HA2, to broader expression as for HY, HA3 and HA4 antigens (Goulmy, 1996; Warren et al., 1998).

Initially, murine skin graft rejection models revealed that a mismatch of MHC molecules between donor and recipient led to rapid graft rejection (Rosenberg and Singer, 1992). The transfer of T cells from a sensitized donor to a naïve recipient accelerated the graft rejection, indicating that T lymphocytes caused this immune response. In experiments where donor and recipient were MHCmatched but differed in miHC antigens, a slower graft rejection was monitored (Rosenberg and Singer, 1992), initially revealing that miHC antigen can influence the success of transplantations. Most available clinical data concerning miHC antigen mismatch in humans were found in the field of hematopoietic stem cell transplantations (HSCT). Here, it was reported that the expression of disparate miHC antigens increased the risk to develop acute graft versus host disease (GvHD) (Dzierzak-Mietla et al., 2012; Goulmy et al., 1996; Santos et al., 2016; Tseng et al., 1999) and chronic GvHD (Dzierzak-Mietla et al., 2012; Markiewicz et al., 2009; Mutis, 2015; Nakasone et al., 2015). Studies about discrepancies in miHC antigen expression in solid organ transplantation are still rare and controversial. For instance patient studies on autosomal MHC class I-restricted miHC antigens in kidney transplantations revealed no effects of minor miHC antigen mismatching (Dierselhuis et al., 2013; Heinold et al., 2008). However, other studies showed an influence of miHC antigen discrepancies on the outcome of transplantation as HY-mismatching affected the clinical outcome of kidney and cornea transplantations (Böhringer et al., 2006; Gratwohl et al., 2008; Tan et al., 2008). The involvement of $\mathrm{CD}^{+} \mathrm{T}$ lymphocytes against $\mathrm{HY}$ antigens was shown to result in plasma cell infiltration into biopsied kidneys and antibody development against miHC antigens, which was consistent with findings of other groups (Porcheray et al., 2011; Spierings et al., 2003). In a study of miHC antigen mismatched skin and hematopoietic cell grafts, the role of direct presentation by donor DCs was evaluated. It revealed that direct presentation of miHC antigens by donor DCs is needed for an efficient rejection of grafts by CTLs but that in the absence of donor DCs an immune response is caused by indirect presentation of antigens (Fernandes et al., 2011). 
In this thesis, it was confirmed that indeed expression of miHC antigens elicit antigen-dependent immune responses mediated by miHC antigen-specific CTLs, helper $\mathrm{T}$ and $\mathrm{B}$ cells. For this an in vivo murine model of miHC mismatch, due to OVA expression in the inoculated stem cells, was used in teratoma formation assays in otherwise syngeneic hosts. Since stem cells and stem cell-derived grafts are a potential source for cell replacement therapies, our findings showing their immunogenicity have to be considered for future replacement therapies. However, the intensity of the influence that miHC antigens have on immune responses might depend on the miHC antigen itself and on the conditions of transplantation. Additionally, the differentiation status and type of transplanted cells might alter the possibility to activate adaptive immune responses against miHC antigens. Nonetheless, the identification of potential immunogenic miHC antigens in PSCs and in PSC-derived grafts would allow screening for functionally relevant incompatibilities between donor and recipient and would be a start to evaluate the immunogenicity of miHC antigens in vivo. At this, potential recipients at risk of graft rejections could be treated by specific immunosuppressive strategies.

\subsection{Killing of murine pluripotent stem cells largely depends on the activating NK receptor NKG2D}

NK cells play an important role as cytotoxic effector cells scanning the organism for stressed, infected or malignant cells. Future strategies to treat diseases, such as HF or Parkinson's disease might include the transplantation of PSC-derived grafts. However, those grafts are at risk of giving rise to teratomas in the recipient, if residuals of undifferentiated cells are still present in the grafts after in vitro differentiation. Previously, it was shown that NK cells are able to kill various human and murine PSCs in xenogeneic, allogeneic and syngeneic experimental set-ups (Dressel et al., 2008, 2010; Kruse, Hamann et al., 2015; Tian et al., 2006). The involvement of NKGD2 receptors on NK cells in killing of PSCs was assumed (Feng et al., 2009; Kim et al., 2007; Ogasawara et al., 2005) and then shown in an inhibition study in our group in which anti-NKG2D antibodies were used for analysis (Dressel et al., $2008,2010)$. To confirm the influence of NKG2D in the killing of various PSCs, a mouse model of NKG2D deficiency was used to test whether the susceptibility of PSCs to NK cells was altered in those animals. Here, the killing of PSCs by NK cells of NKG2D-deficient mice was compared to NK cells derived from wildtype C57BL/6 mice. All analyzed PSCs, including syngeneic and allogenic (but MHC-matched) maGSCs, ESCs and iPSCs, showed cell surface expression of NKG2D ligands which made them to potential targets for NK cell killing. The important role of NKG2D in the killing of those PSCs was then 
clarified in in vitro cytotoxicity assays, in which it was shown that all PSCs were largely resistant to killing by naïve NKG2D-deficient NK cells. At this, wildtype NK cells showed a moderate level of killing even without previous in vitro stimulation of the NK cells. However, the stimulation of NK cells with IL-2 led to an increased killing of the target cell lines by NKG2D-deficient and wildtype NK cells. Nonetheless, IL-2-activated wildtype NK cells still showed higher lysis rates than NKG2D-deficient NK cells. This indicates that the moderate killing of PSCs largely depends on NKG2D receptors if NK cells are not stimulated. After stimulation of NK cells, obviously also other pathways than NKG2D are used, leading to an increased but not fully reconstituted killing of PSCs when compared to wildtype NK cell killing. Other ligand-receptor interactions that partially compensate the absent NKG2D receptor might include the activating NK cell receptor DNAM-1. The ligands for DNAM-1 that are CD112 and CD155 were found to be expressed on human and murine stem cells (Dressel et al., 2010; Kruse, Hamann et al., 2015). Examination of human iPSC susceptibility to NK cells revealed that here DNAM-1 was the main mediator of stem cell killing by NK cells, albeit in a single donor-target combination also NKG2D was shown to influence PSC susceptibility (Kruse, Hamann et al., 2015). Since only one inbred mouse strain was analyzed in this thesis, it is possible that the NK cell receptor-ligand interactions are more diverse amongst different strains with different genetic background, which was already indicated for humans (Kruse, Hamann et al., 2015).

In conclusion, it was demonstrated that NKG2D is an important activating receptor involved in killing of murine PSCs, which was lately also shown to concern other stem cell-like tumors (Rong et al., 2016; Wei et al., 2015; Zhang et al., 2016). Consequently, this knowledge can be used to develop strategies to deplete contaminating PSCs before grafting, thereby increasing the safety of transplantations. Moreover, immunosuppressive therapies not affecting NK cells might be favorable, if allogeneic PSCderived grafts are transplanted to allow a killing of residual PSCs by the NK cells of the recipient. 


\section{Summary and Conclusions}

In this thesis, the effect of immune responses on the pathogenesis of load-induced HF and the rejection of stem cell and stem cell-derived grafts was analyzed. It was shown that an increased afterload in a murine model of HF was sufficient to activate T helper cells with a specificity for an antigen expressed on cardiomyocytes and to accelerate the progression to HF in TCR-transgenic animals at risk to develop autoimmunity. T helper cells exerted their effect on the heart function independently of autoantibody induction. Thus, autoimmunity may contribute to the transition from hypertrophy to HF even when caused by an increased afterload. Immune reactions against cardiac antigens might also affect the chances to treat HF by new PSC-based therapies. The expression of a single miHC antigen in PSCs affected the engraftment in otherwise syngeneic hosts due to activation of adaptive immune responses including antigen-specific CTLs, Thelper and B cells. Additionally, it was demonstrated that various stem cells became targets for NK cells and that NKG2D was an important activating receptor involved in killing of murine PSCs. These findings are important for future therapeutic applications, e.g. to prevent activation of autoimmune T cells in heart failure patients. Moreover, variant miHC antigen expression in transplantations between donor and recipients have to be considered. Consequently, a suitable immunosuppressive or immunomodulatory therapy primarily targeting adaptive immune cells is needed. NK cells might be important to eliminate residual PSCs after transplantation of stem-cell derived grafts. 


\section{Supplement}

\subsection{Investigation of immune response contributing to the pathogenesis of load-induced heart failure}

\subsubsection{Leukocyte cell composition of spleen and heart}

The splenocytes of animals which received a mixture of OT-I and OT-II T cells were further analyzed via flow cytometry to determine the proportion of myeloid cells and lymphocytes in the population. In total, $81.6 \%$ (sham) to $82.8 \%$ (TAC) of splenocytes were positive for the leukocyte marker CD45. This population of $\mathrm{CD} 45^{+}$cells included $\mathrm{B}$ cells $\left(\mathrm{CD} 19^{+} \mathrm{CD} 45 \mathrm{R}^{+}\right)$, T cells $\left(\mathrm{CD}^{+} \mathrm{CD}^{+}, \mathrm{CD}^{+} \mathrm{CD} 8^{+}\right)$, macrophages $\left(\mathrm{F} 4 / 80^{+}\right)$, NKT cells $\left(\mathrm{CD}^{+} \mathrm{DX} 5^{+}\right)$, dendritic cells $\left(\mathrm{CD} 11 \mathrm{c}^{+} \mathrm{MHCI}+\mathrm{I}^{+}\right)$, NK cells $\left(\mathrm{CD}^{-} \mathrm{DX} 5^{+}\right)$, neutrophils $\left(\mathrm{CD} 11 \mathrm{~b}^{+} \mathrm{Ly}_{6 \mathrm{G}^{+}}\right)$, and monocytes $\left(\mathrm{CD} 115^{+} \mathrm{C} 11 \mathrm{~b}^{+} \mathrm{Ly} 6 \mathrm{C}^{+}\right)$listed in decreasing order of cell numbers (Figure 60A). Most abundant cells were $B$ cells which made up almost half of the leukocyte population (41.6 \% (TAC) to $44 \%$ (sham)) followed by T helper cells (16.2\% (sham) to $19.2 \%$ (TAC)), cytotoxic T cells $(9.5 \%$ (sham) to $10.7 \%$ (TAC)) and macrophages (9.1\% (TAC) to $9.3 \%$ (sham)). The cell populations with lower cell numbers included NK T cells (1.3\% (TAC) to $3.1 \%$ (sham)), dendritic cells (2.2\% (sham) to $2.3 \%$ (TAC)), NK cells (0.9\% (sham) to $1.6 \%$ (TAC)), neutrophils $(1.1 \%$ (sham) to $1.2 \%(T A C))$ and monocytes (0.4\%). Analysis of the proportion of $\mathrm{CD} 4{ }^{+} \mathrm{CD} 45.1^{+}$cells in the leukocyte population of cMy-mOVA mice was determined, showing that $0.4 \%$ of $\mathrm{CD}_{4} 5^{+}$cells derived from OT-II mice $\left(\mathrm{CD} 45.1^{+}\right)$, transferred via adoptive transfer. However, no significant differences in the splenocyte composition between TAC- and sham-operated animals were found.

Additionally, heart tissue was digested to single cells and analyzed via flow cytometry to clarify the proportion of lymphocytes and myeloid cells in the target tissue itself. In hearts of TAC-operated animals $6.5 \%$ and in sham-operated animals $3.4 \%$ of cells were positive for CD45. Most abundant leukocytes in the myocardium of cMy-mOVA mice were macrophages, whose cell proportions ranged from $35.6 \%$ (TAC) to $53.2 \%$ (sham) of the total leukocyte population (Figure 60B). B cells, which were found in significantly higher levels in the heart after TAC operation (13\% (sham) to $15 \%$ (TAC); $p=0.01$, student's t-test) and monocytes (12.3 (TAC) to $19.2 \%$ (sham)) were found in moderate numbers, followed by dendritic cell population ( $5.2 \%$ (TAC) to $8.3 \%$ (sham); $p=0.02$, student's t-test). Thelper cells and neutrophils made up around 4 to $5 \%$ of leukocyte population (Thelper: $3.5 \%$ (sham) to $5.5 \%$ (TAC); neutrophils: $3.6 \%$ (TAC) to $3.9 \%$ (sham)). Populations of cytotoxic T cells, NK cells and NKT cells were detected in lower cell numbers ranging from $0.4 \%$ (TAC) to $0.6 \%$ (sham) for NKT cells, $0.9 \%$ (sham) to $1.6 \%$ (TAC) for NK cells and from $1.3 \%$ (sham) to $2.4 \%$ (TAC) for CD8 ${ }^{+}$T cells. The 
amount of $\mathrm{CD} 45.1^{+} \mathrm{CD} 4^{+}$cells derived from transferred OT-II cells was monitored to be significantly higher in TAC -(3\%) than sham- (1\%) operated animals ( $p=0.02$, student's t-test).

Generally, no major differences of the lymphocyte composition in the spleen and myocardium of TACoperated animals compared to sham mice were found after the animals received adoptive transfer of OT-I and OT-II T cells, besides an increase in B cell numbers in the myocard. However, TAC-operated animals displayed a higher amount of $\mathrm{CD} 45^{+}$positive cell in the heart tissue, suggesting activation of inflammatory responses which led to an increased recruitment of leukocytes into the heart tissue.
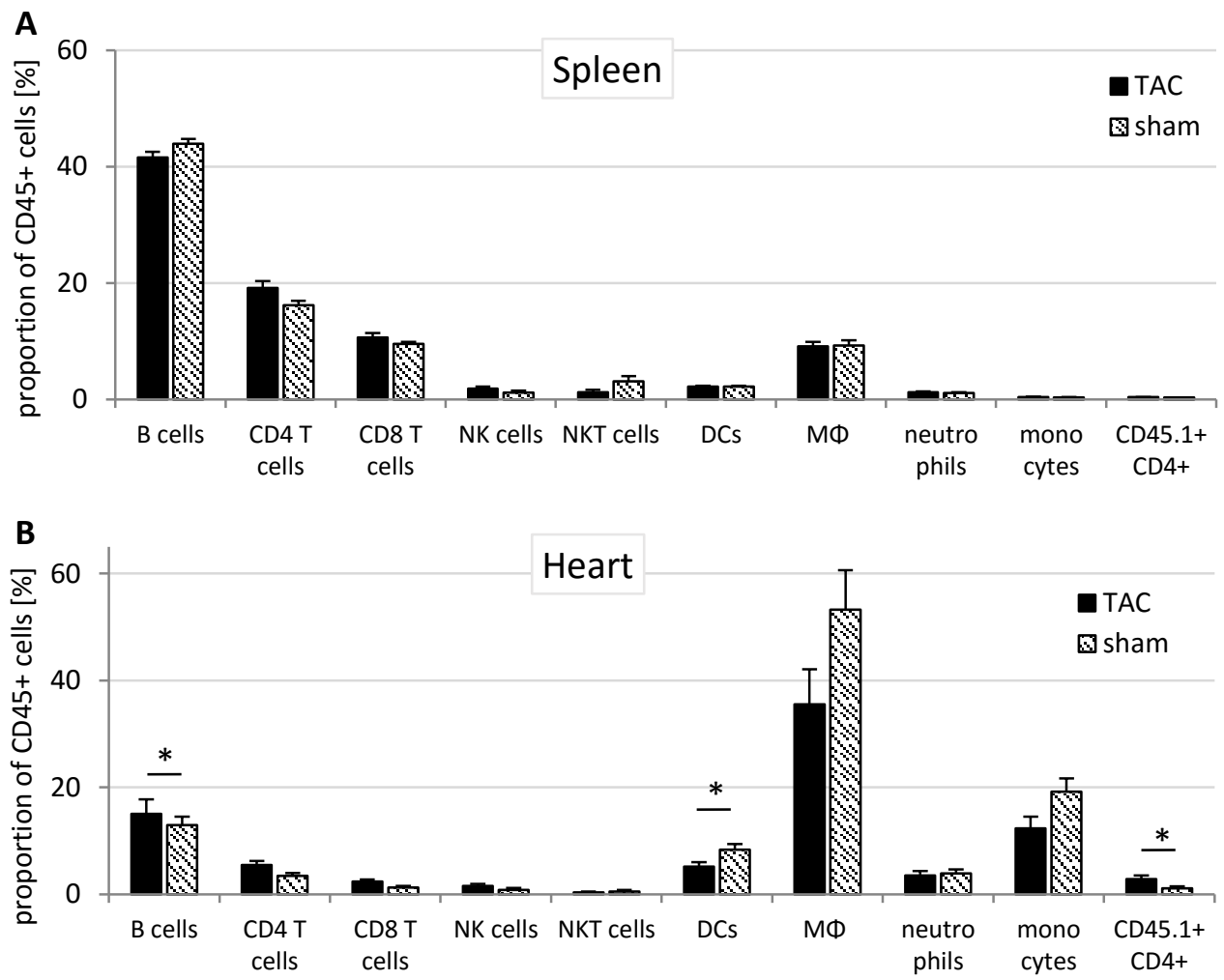

Figure 60 Proportion of lymphocytes and myeloid cells in the (A) spleen and (B) myocardium of cMy-mOVA mice which received OT-I and OT-II TCR-specific $T$ cells.

Shown is the mean proportion of $\mathrm{B}$ cells $\left(\mathrm{CD} 19^{+} \mathrm{B}^{\left.22 \mathrm{O}^{+}\right)}\right.$, $\mathrm{T}^{\mathrm{c}}$ cells $\left.\left(\mathrm{CD3}^{+}, \mathrm{CD}^{+} / \mathrm{CD}^{+}\right)\right)$, NK cells $\left(\mathrm{CD}^{-}\right.$ $\left.\mathrm{DX} 5^{+}\right)$, neutrophils $\left(\mathrm{CD} 11 \mathrm{~b}^{+} L y 6 \mathrm{G}^{+}\right)$, patrolling monocytes $\left(\mathrm{CD} 115^{+} \mathrm{C} 11 \mathrm{~b}^{+} \mathrm{Ly} 6 \mathrm{C}^{\text {low }}\right)$, inflammatory monocytes $\left(C D 115^{+} C 11 b^{+}\right.$Ly6 $\left.C^{\text {high }}\right)$, macrophages $\left(F 4 / 80^{+}\right)$and dendritic cells $\left(C D 11 c^{+} I-A b\right)$ of the CD45 $5^{+}$population + SEM. TAC $(n=7)$, sham $(n=6)$ except for T and NK cells: TAC $(n=6)$, sham $(n=$ 3). ${ }^{*}<0.05$, student's t-test 


\subsubsection{Endothelial cells in the myocard}

Moreover, the amount of endothelial cells $\left(\mathrm{CD} 144^{+} \mathrm{CD} 31^{+}\right)$in the population of $\mathrm{CD} 45^{-}$cells in the spleen (as control) and myocardium of the analyzed cMy-mOVA mice was determined to examine the proportion of vascular cell adhesion protein 1 (VCAM-1) and intercellular adhesion molecule 1 (ICAM-1) positive cells. These proteins are known to be important in inflammation and immune responses and could be responsible for an increased infiltration into the heart, if upregulated on cardiac endothelial cells. In the analyzed splenocyte population, $7.4 \%$ (TAC) to $16.7 \%$ (sham) were negative for the CD45 marker whereas in the myocardium $90.5 \%$ (TAC) to $96.3 \%$ (sham) of cells were not expressing this leukocyte marker. Epithelial cells positive for CD144 and CD31 in the CD45population ranged from $0.3 \%$ (TAC) to $0.6 \%$ (sham) in the spleen and from $9.3 \%$ (sham) to $9.8 \%$ (TAC) in heart tissue (Figure 61) Further analysis of $\mathrm{CD} 144^{+} \mathrm{CD} 31^{+}$cells showed that most of the cells in the spleen were double positive for VCAM-1 and ICAM-1 (39.5\% (TAC) to $45.2 \%$ (sham)), and around 5 to $10 \%$ positive for single proteins. In contrast, in heart tissue around $50 \%$ of the $C D 144^{+} \mathrm{CD} 31^{+}$cells were single positive for ICAM-1. Only $22.1 \%$ (TAC) to $22.7 \%$ (sham) of CD $144^{+}$CD $31^{+}$cells were double positive for ICAM-1 and VCAM-1. VCAM-1 expression was found only on $1.9 \%$ (TAC) to $2.5 \%$ (sham) in this cell population.

Overall, adoptive transfer and TAC operation was not associated with an altered expression in VCAM1 or ICAM-1 on epithelial cells compared to sham-operated animals.

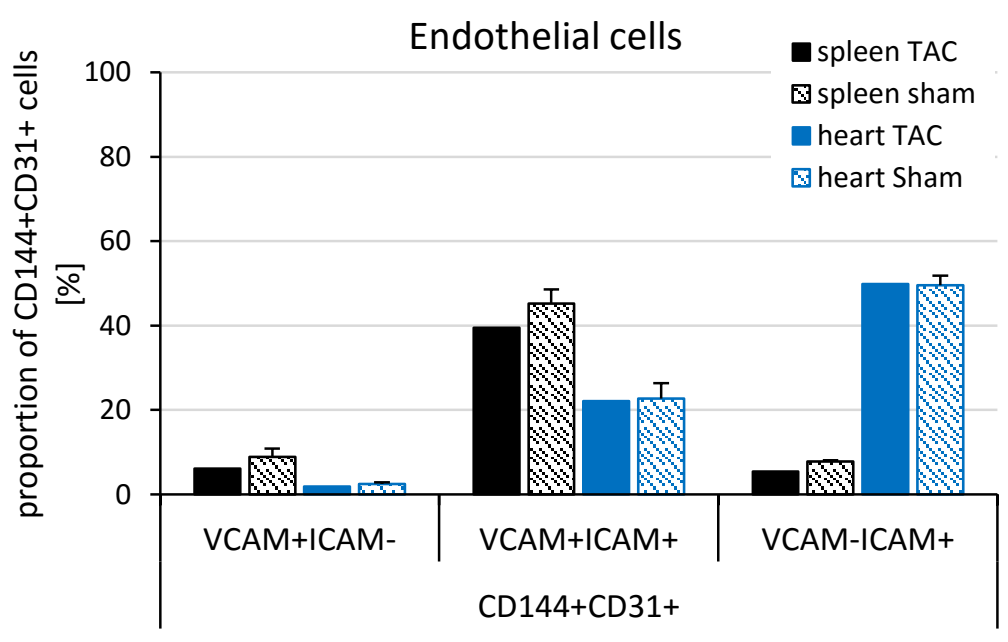

Figure 61 Flow cytometric analysis of intercellular adhesion molecules ICAM-1 and VCAM-1 on endothelial

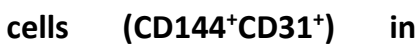
spleen and heart of cMymOVA mice which received a mixture of OT-I and OT-II T cells.

Shown is the mean proportion + SEM. TAC $(\mathrm{n}=$ $1)$, sham $(n=3)$. 


\subsection{Stem cell immunogenicity}

\subsubsection{Germ layer marker expression in tumors derived from wildtype and OVA-expressing stem cells in immunodeficient and immunocompetent host}

Besides the characterization of tumors as teratomas by histology, qPCR analysis was performed to examine germ layer marker gene expression in various tumors, confirming the formation of teratomas after the inoculation of various stem cell clones into immunodeficient (summarized as RAG2 ${ }^{-{ }^{-}} \mathrm{Yc}^{-{ }^{-}}$) and immunocompetent (129/Sv) mice. As germ layer markers for the analysis served Th and Syn as marker for ectodermal structures, $A f p$ and $A l b$ for endodermal tissues and Ctnt and $\alpha M h c$ for mesodermal tissues in the tumor. These markers are not expressed on stem cell lines (data not shown) and therefore tissues of syngeneic mice (129/Sv) known to be positive for the specific germ layer markers were used as reference cells including hippocampal tissue for ectodermal, spleen and liver for endodermal and lung tissue for mesodermal germ layer markers. In this analysis, Hprt and Gapdh were used as housekeeper genes. In Figure 62, the mRNA expression of germ layer markers are summarized for tumors derived from wildtype or OVA-expressing stem cells in immunocompetent or immunodeficient mice. The OVA-expressing stem cells consisted of iPSC 129/Sv clone OVA\#6 and OVA\#24, whereas ESC BTL1 and iPSC 129/Sv TD11.1 were injected as wildtype cell lines. The analyzed tumors showed a variable expression of the tested germ layer markers of which at least one out of two for every germ layer was clearly detectable. Consequently, the tumors could be characterized as teratomas. Expression of markers showed a high standard derivation due to variations in different teratomas, indicating that the differentiation is random. Each teratoma showed a variable stage of differentiation with different amounts of cells of each germ layer. Overall, Th and Afp were the most abundant expressed markers in teratomas derived from OVA-expressing stem cells and wildtype stem cells in RAG2 ${ }^{-1} \mathrm{\gamma c}^{-/-}$and 129/Sv mice, with even higher expression than in the control tissues. Ctnt was rather expressed in teratomas formed in immunodeficient animals (1.4 and 2.1 fold of reference tissue) than in immunocompetent hosts ( 0.05 to 0.08 fold). Syn and $\alpha M H C$ were only expressed at low levels (Syn: 0.03 to 0.33 fold; $\alpha M H C$ : 0.02 to 0.48 fold), whereas $A l b$ was not detectable in any teratoma. 


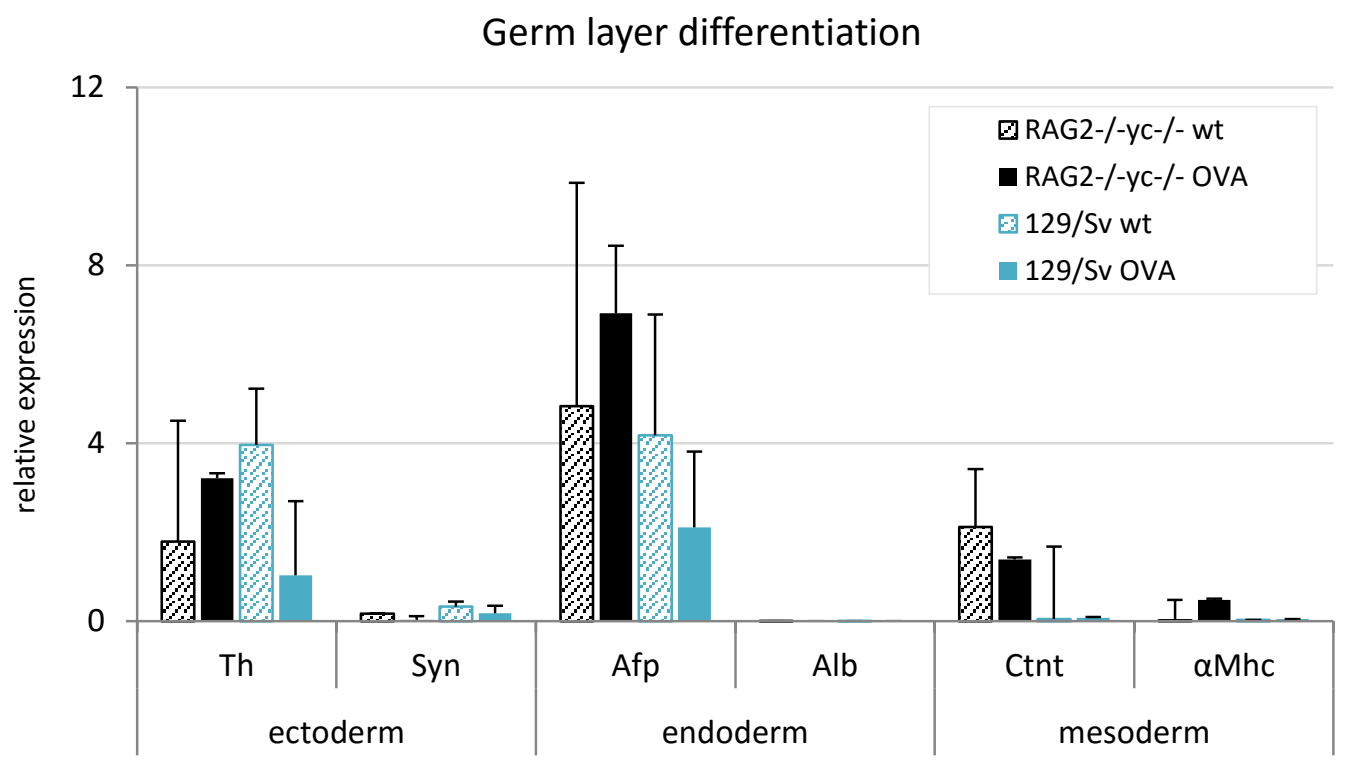

Figure 62 Germ layer marker expression in teratomas.

Shown is the mean relative expression of germ layer marker to their reference tissues known to be positive for each gene: ectoderm (Th, Syn), endoderm (Afp, Alb), mesoderm (Ctnt, $\alpha M h c$ ) of 129/Sv mice + SD. Mean of Hprt and Gapdh was used as housekeeper reference. OVA: iPSC 129Sv \#6, \#24; wt: ESC BTL1, iPSC 129/Sv. Analyzed teratomas each $(n=4)$. 


\subsubsection{Influence of adoptive transfer on tumor characteristics in RAG2 ${ }^{-/-} \mathrm{\gamma c}^{-/-}$mice}

The adoptive transfer of TCR-transgenic T cells had no significant effects on the size or weight of tumors derived by wildtype or OVA-expressing stem cells in immunodeficient mice. In Figure 63, it is shown that those parameters varied between tumors, independent of OVA expression and type of T cell transfer.
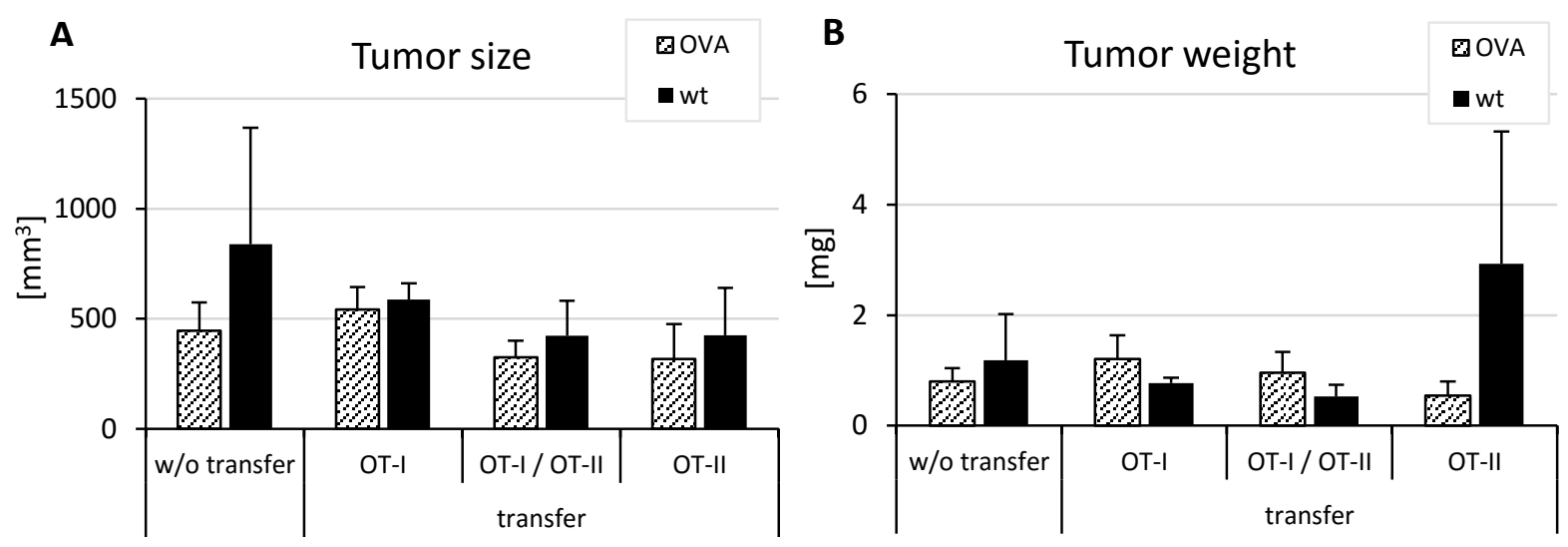

Figure 63 Analysis of (A) tumor size and (B) tumor weight after injections of OVA-expressing and wildtype stem cells into immunodeficient hosts which received either TCR-transgenic T cells (OT-I T cells, OT-II T cells, OTI/OT-II T cells) or no $T$ cells (w/o transfer).

(A) Depicted is the mean tumor size + SEM. W/o transfer: OVA $(n=23)$, wt $(n=6)$; OT-I: OVA $(n=10)$, wt $(n=3)$; OT-I/OT-II: OVA $(n=12)$, wt $(n=5)$; OT-II: OVA $(n=3)$, wt $(n=2)$. Differences in tumor size between tumors derived from OVA-expressing stem cells $(p=0.32)$, between tumors derived from wildtype stem cells $(p=0.71)$, Kruskal-Wallis H-test. (B) Shown is the mean tumor weight + SEM. W/o transfer: OVA $(n=23)$, wt $(n=5)$; OT-I: OVA $(n=11)$, wt $(n=3)$; OT-I/OT-II: OVA $(n=14)$, wt $(n=5)$; OT-II: OVA $(n=3)$, wt $(n=3)$. Differences in tumor weight between OVA-expressing stem cell derived tumors $(p=0.36)$, between wildtype stem cell derived tumors $(p=0.51)$; Kruskal-Wallis H-test. 


\subsubsection{Influence of HY antigens on tumor formation in immunocompetent mice}

Further analysis of tumor formation for each stem cell clone in hosts of different gender revealed that almost all male stem cell lines showed tumor formation in male recipients but did not engraft in female hosts. Only injections of ESC BTL1 OVA\#4 led to tumor formation in female hosts (Figure 64). However, tumor formation rate was only significantly altered for two male iPS cell clones (iPSC OVA\#6, \#24) which showed a high tumor formation rate in male host, but in contrast, no tumor formation in female hosts. Therefore, an additional influence of other miHC antigens derived from the Y-chromosome of male stem cells on the rejection of OVA-expressing stem cell tumors cannot be excluded.

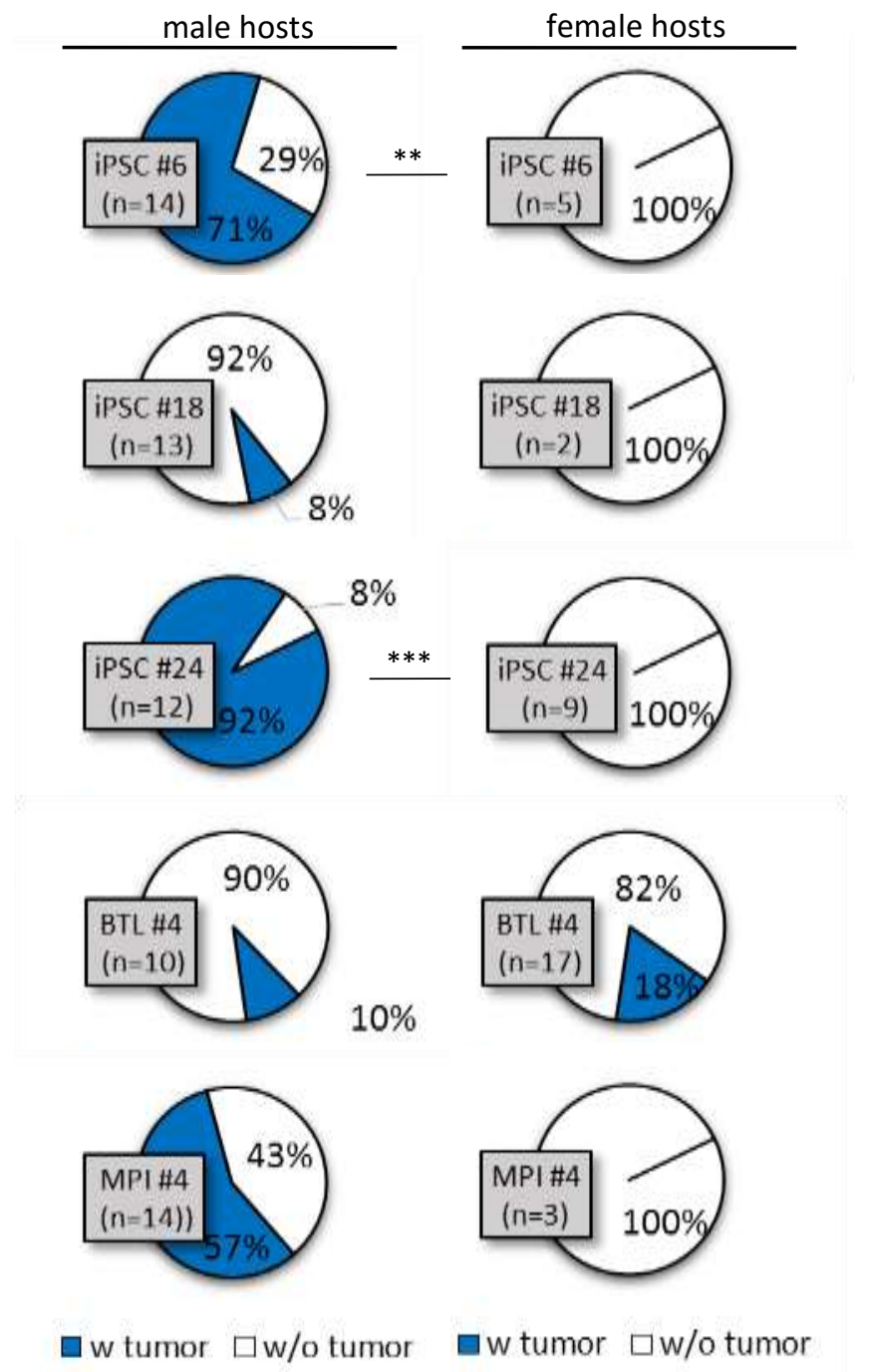

Figure 64 Tumor formation after injection of OVA-expressing stem cells into immunocompetent $129 /$ Sv mice of different sex.

IPSC OVA\#13 were not injected into female hosts, and are consequently not depicted in the graph. Numbers of experiments are depicted in each diagram. Significant differences in tumor formations between male and female hosts are marked with asterisk. $* *<0.01 ; * * *<0.001$. IPSC OVA\#6 ( $p=6$ $\left.x 10^{-3}\right)$, iPSC OVA\#18 ( $\left.p=0.68\right)$, iPSC OVA\#24 $\left(p=3.2 \times 10^{-5}\right)$, ESC BTL1 OVA\#4 ( $p=0.59)$, ESC MPI-II OVA\#4 ( $p$ $=0.07)$; chi-squared test. 


\subsubsection{Leukocyte infiltration into teratomas analyzed by flow cytometry}

For flow cytometry, tumors were digested to single cells and analyzed for several leukocyte populations. As seen in Figure 65, tumors derived from wildtype and OVA-expressing stem cells were positive for T cells $\left(\mathrm{CD}^{+}, \mathrm{CD} 4^{+}, \mathrm{CD} 8^{+}\right)$, B cells $\left(\mathrm{CD} 45 \mathrm{R}^{+}\right)$, macrophages $\left(\mathrm{F} 4 / 80^{+}\right)$, dendritic cells $\left(\mathrm{CD} 11 \mathrm{c}^{+}\right)$ and NK cells $\left(\mathrm{NKp}^{+} 6^{+}, \mathrm{DX} 5^{+}\right)$. Numbers of cytotoxic T cells, dendritic cells and macrophages appeared to be higher in OVA-derived tumors compared to wildtype tumors, ranging from 10 to $30 \%$ of CD45 cells. In contrast, wildtype stem cell-derived teratomas showed higher numbers of $\mathrm{T}$ helper cells (22.6\%), B cells (10\%), NKp46 ${ }^{+}(26.7 \%)$ and DX5 $5^{+}(11.2 \%)$ NK cells compared to OVA-expressing tumors (CD3 ${ }^{+} \mathrm{CD}^{+}: 14.2 \%, \mathrm{CD}^{+} 5 \mathrm{R}^{+}: 8 \%, \mathrm{NKp}^{2} 6^{+}: 20.8 \%, \mathrm{DX5}^{+}: 6.8 \%$ ). Additionally, cells positive for the TCR $V \beta$ 5.1/5.2 were found, but in significantly higher numbers in teratomas derived from OVA-expressing stem cells $(16.6 \%)$ compared to tumors formed by wildtype stem cells ( $3 \% ; p=0.047$ ). T cells using this receptor can be OVA-specific T cells as demonstrated by the OT-I and OT-II TCR. However, whether this indicates reactivity of OVA-specific T cells against the OVA expressed in the tumor tissue remains speculative.

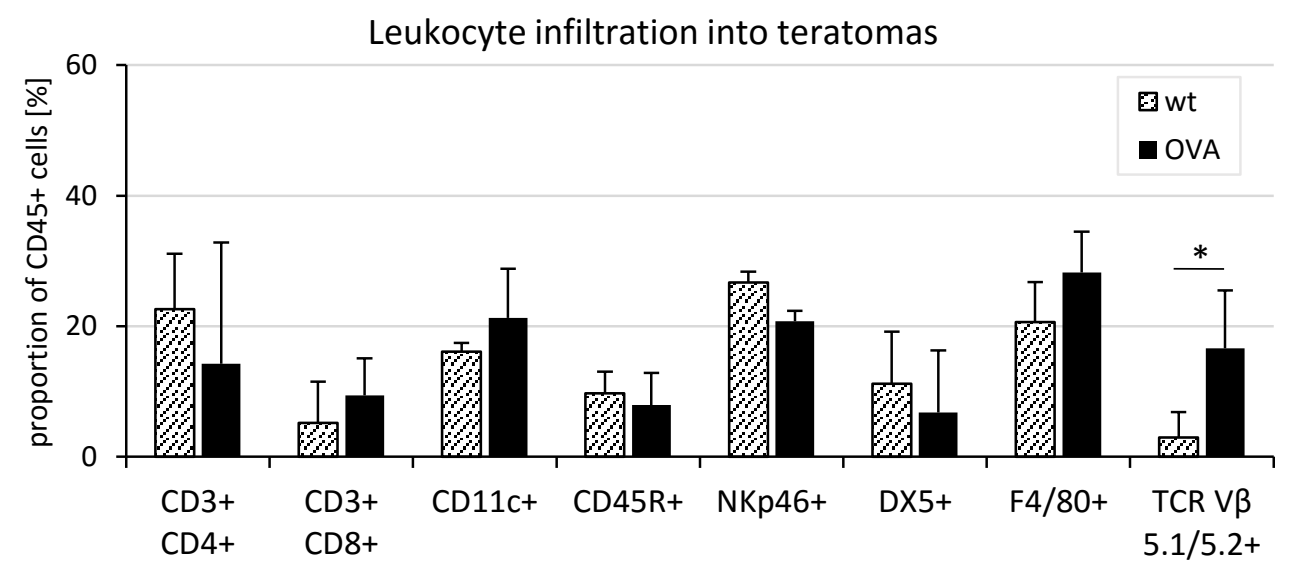

Figure 65 Analysis of leukocyte infiltration into teratomas analyzed by flow cytometry. Leukocyte population of $\mathrm{CD}^{4} 5^{+}$cells in tumor tissue derived from wildtype and OVA-expressing stem cells in syngeneic hosts $(129 / S v)+S E M$. ESC BTL1 wt $(n=6)$, iPSC OVA $(n=2)$. Differences between teratomas derived from wt and OVA-expressing stem cells: $\mathrm{CD}^{+} \mathrm{CD}^{+}(p=0.5), \mathrm{CD}^{+} \mathrm{CD}^{+}(p=0.36)$, $\operatorname{CD}_{11} c^{+}(p=0.45), \operatorname{CD}_{5} R^{+}(p=0.58),{\operatorname{NKp} 46^{+}}(p=0.71), D_{X} 5^{+}(p=0.71), F 4 / 80^{+}(p=0.39), T C R$ V $\beta$ $5.1 / 5.2^{+}(p=0.047)$; student's t-test. 


\subsubsection{Cellular composition of lymphocytes of host splenocytes after stem cell injections}

Splenocytes obtained from 129/Sv hosts which previously received injections of wildtype and OVAexpressing stem cells were further analyzed to determine their cellular composition via flow cytometry. For this, freshly isolated (day 0 ) splenocytes of animals which showed tumor growth or failed to form teratomas were used. As seen in Figure 66, most abundant cell types were Thelper cells $\left(\mathrm{CD}^{+}{ }^{+} \mathrm{CD} 4^{+}\right.$: around $\left.25 \%\right)$ and $\mathrm{B}$ cells $\left(\mathrm{CD} 45 \mathrm{R}^{+}\right.$: around $\left.35 \%\right)$, followed by cytotoxic $\mathrm{T}$ cells $\left(\mathrm{CD} 3^{+} \mathrm{CD} 8^{+}\right.$: around $10 \%$ ). Additionally, a minor extent of these T cells was positive for the TCR V $\beta$ 5.1/5.2 which recognizes OVA, and some NK cells $\left(\mathrm{DX}^{+} \mathrm{CD}^{-} \mathrm{NKp}^{-} 6^{+}\right)$were found. In summary, no differences in the lymphocyte composition of animals that received OVA-expressing stem cells compared to wildtype stem cells could be monitored. Additionally, the lymphocyte composition of animals which rejected the stem cells resembled those of animals in which the injected stem cells engrafted.

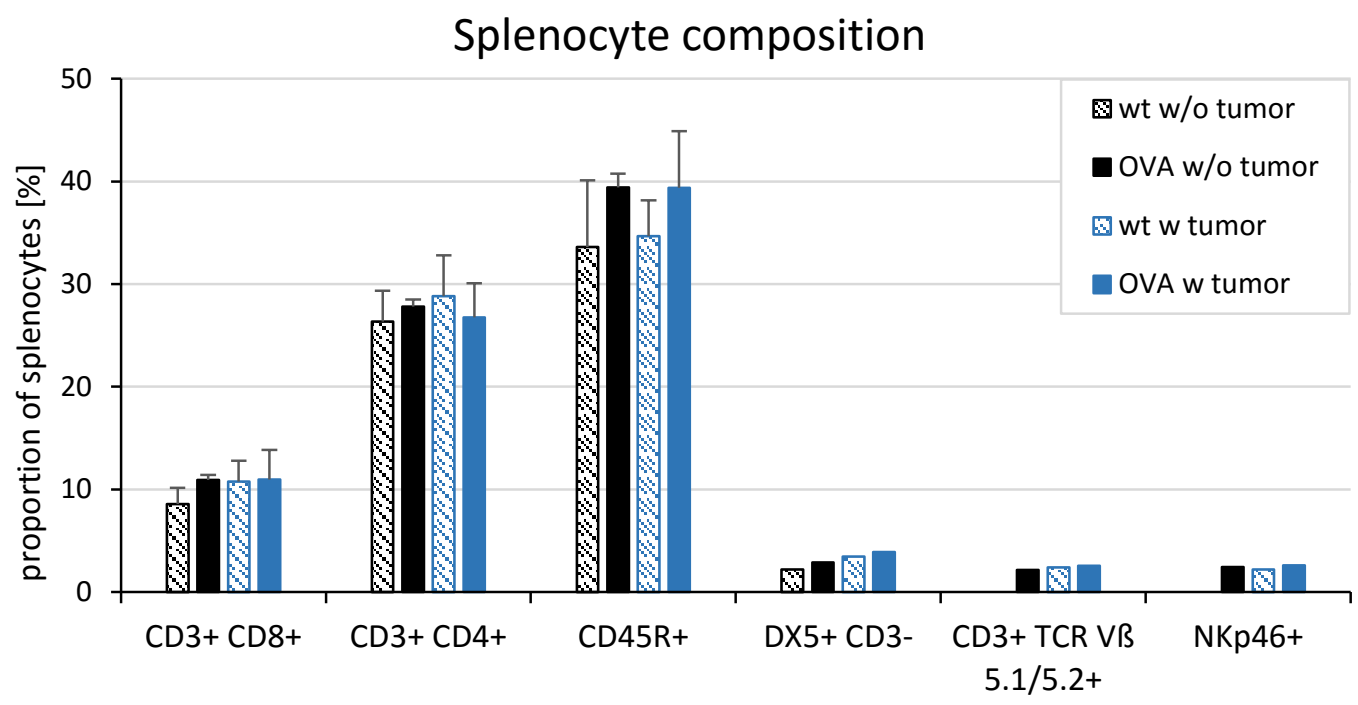

Figure 66 Naïve splenocyte composition of animals which received either OVA-expressing (OVA) or wildtype (wt) stem cells and which showed tumor formation (w tumor) or tumor rejection (w/o tumor).

Shown is the mean proportion of splenocytes + SEM. Wt w/o tumors: $\mathrm{CD}^{+} \mathrm{CD} 8^{+}(n=5), \mathrm{CD}^{+} \mathrm{CD} 4^{+}(n$

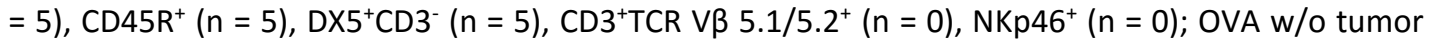

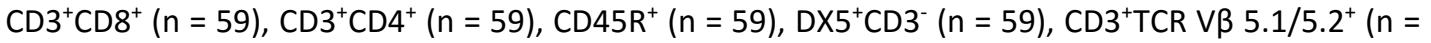
15), $\mathrm{NKp}^{+} 6^{+}(\mathrm{n}=15)$; wt w tumor: $\mathrm{CD3}^{+} \mathrm{CD}^{+}(\mathrm{n}=12), \mathrm{CD3}^{+} \mathrm{CD}^{+}(\mathrm{n}=12), \mathrm{CD}^{+} 5 \mathrm{R}^{+}(\mathrm{n}=12), \mathrm{DX5}^{+} \mathrm{CD3}^{-}(\mathrm{n}$

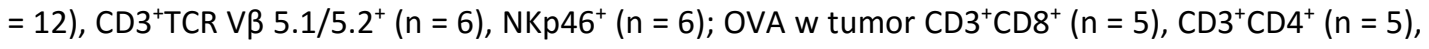
$\operatorname{CD}_{5} R^{+}(n=5), \operatorname{DX5}^{+} \mathrm{CD}^{-}(n=5), \mathrm{CD}^{+} \mathrm{TCR} V \beta 5.1 / 5.2^{+}(n=3),{\mathrm{NKp} 46^{+}}^{(}(n=3)$. 


\section{Bibliography}

Abdullah, Z., Saric, T., Kashkar, H., Baschuk, N., Yazdanpanah, B., Fleischmann, B.K., Hescheler, J., Krönke, M., and Utermöhlen, O. (2007). Serpin-6 expression protects embryonic stem cells from lysis by antigenspecific CTL. J. Immunol. Baltim. Md 1950 178, 3390-3399.

Abou-Raya, A., and Abou-Raya, S. (2006). Inflammation: a pivotal link between autoimmune diseases and atherosclerosis. Autoimmun. Rev. 5, 331-337.

Actor, J.K. (2014). Introductory Immunology: Basic Concepts for Interdisciplinary Applications (Academic Press).

Ademokun, A.A., and Dunn-Walters, D. (2010). Immune Responses: Primary and Secondary. In eLS, John Wiley \& Sons, Ltd, ed. (Chichester, UK: John Wiley \& Sons, Ltd),.

Anokye-Danso, F., Trivedi, C.M., Juhr, D., Gupta, M., Cui, Z., Tian, Y., Zhang, Y., Yang, W., Gruber, P.J., Epstein, J.A., et al. (2011). Highly efficient miRNA-mediated reprogramming of mouse and human somatic cells to pluripotency. Cell Stem Cell 8, 376-388.

Araki, R., Uda, M., Hoki, Y., Sunayama, M., Nakamura, M., Ando, S., Sugiura, M., Ideno, H., Shimada, A., Nifuji, A., et al. (2013). Negligible immunogenicity of terminally differentiated cells derived from induced pluripotent or embryonic stem cells. Nature 494, 100-104.

Ascherio, A., and Munger, K.L. (2007). Environmental risk factors for multiple sclerosis. Part II: Noninfectious factors. Ann. Neurol. 61, 504-513.

Awe, J.P., Gschweng, E.H., Vega-Crespo, A., Voutila, J., Williamson, M.H., Truong, B., Kohn, D.B., Kasahara, N., and Byrne, J.A. (2015). Putative immunogenicity expression profiling using human pluripotent stem cells and derivatives. Stem Cells Transl. Med. 4, 136-145.

Baldeviano, G.C., Barin, J.G., Talor, M.V., Srinivasan, S., Bedja, D., Zheng, D., Gabrielson, K., Iwakura, Y., Rose, N.R., and Cihakova, D. (2010). Interleukin-17A is dispensable for myocarditis but essential for the progression to dilated cardiomyopathy. Circ. Res. 106, 1646-1655.

Barnden, M.J., Allison, J., Heath, W.R., and Carbone, F.R. (1998). Defective TCR expression in transgenic mice constructed using cDNA-based alpha- and beta-chain genes under the control of heterologous regulatory elements. Immunol. Cell Biol. 76, 34-40.

Bendjelid, K., and Pugin, J. (2004). IS dressler syndrome dead?*. Chest 126, 1680-1682.

Blankesteijn, M., and Altara, R. (2014). Inflammation in Heart Failure (Boston, MA: Academic Press).

Bleakley, M., and Riddell, S.R. (2004). Molecules and mechanisms of the graft-versus-leukaemia effect. Nat. Rev. Cancer 4, 371-380.

Böhringer, D., Spierings, E., Enczmann, J., Böhringer, S., Sundmacher, R., Goulmy, E., and Reinhard, T. (2006). Matching of the minor histocompatibility antigen HLA-A1/H-Y may improve prognosis in corneal transplantation. Transplantation 82, 1037-1041. 
Bolli, R., Bhatti, Z.A., Tang, X.L., Qiu, Y., Zhang, Q., Guo, Y., and Jadoon, A.K. (1997). Evidence that late preconditioning against myocardial stunning in conscious rabbits is triggered by the generation of nitric oxide. Circ. Res. 81, 42-52.

Bonde, S., and Zavazava, N. (2006). Immunogenicity and engraftment of mouse embryonic stem cells in allogeneic recipients. Stem Cells Dayt. Ohio 24, 2192-2201.

Bönner, F., Borg, N., Burghoff, S., and Schrader, J. (2012). Resident Cardiac Immune Cells and Expression of the Ectonucleotidase Enzymes CD39 and CD73 after Ischemic Injury. PLOS ONE 7, e34730.

Borghi, C., Del Corso, F., Faenza, S., and Cosentino, E. (2015). ACE Inhibitor and Renin-Angiotensin System the Cornerstone of Therapy for Systolic Heart Failure. In ACEi and ARBS in Hypertension and Heart Failure, P. Perrone Filardi, ed. (Cham: Springer International Publishing), pp. 41-72.

Boyd, A.S., and Wood, K.J. (2009). Variation in MHC expression between undifferentiated mouse ES cells and ES cell-derived insulin-producing cell clusters. Transplantation 87, 1300-1304.

Bradley, A., Evans, M., Kaufman, M.H., and Robertson, E. (1984). Formation of germ-line chimaeras from embryo-derived teratocarcinoma cell lines. Nature 309, 255-256.

Brandacher, G., Perathoner, A., Ladurner, R., Schneeberger, S., Obrist, P., Winkler, C., Werner, E.R., Werner-Felmayer, G., Weiss, H.G., Göbel, G., et al. (2006). Prognostic value of indoleamine 2,3dioxygenase expression in colorectal cancer: effect on tumor-infiltrating T cells. Clin. Cancer Res. Off. J. Am. Assoc. Cancer Res. 12, 1144-1151.

Briscoe, D.M., and Sayegh, M.H. (2002). A rendezvous before rejection: where do T cells meet transplant antigens? Nat. Med. 8, 220-222.

Bronte, V., and Zanovello, P. (2005). Regulation of immune responses by L-arginine metabolism. Nat. Rev. Immunol. 5, 641-654.

Buehr, M., Meek, S., Blair, K., Yang, J., Ure, J., Silva, J., McLay, R., Hall, J., Ying, Q.-L., and Smith, A. (2008). Capture of authentic embryonic stem cells from rat blastocysts. Cell 135, 1287-1298.

Calder, P.C. (2006). Branched-chain amino acids and immunity. J. Nutr. 136, 2885 - 935.

Campisi, J. (2005). Senescent cells, tumor suppression, and organismal aging: good citizens, bad neighbors. Cell 120, 513-522.

Cantz, T., Bleidissel, M., Stehling, M., and Schöler, H.R. (2008). In vitro differentiation of reprogrammed murine somatic cells into hepatic precursor cells. Biol. Chem. 389, 889-896.

Caspi, O., Huber, I., Kehat, I., Habib, M., Arbel, G., Gepstein, A., Yankelson, L., Aronson, D., Beyar, R., and Gepstein, L. (2007). Transplantation of human embryonic stem cell-derived cardiomyocytes improves myocardial performance in infarcted rat hearts. J. Am. Coll. Cardiol. 50, 1884-1893.

Chen, H.-F., Yu, C.-Y., Chen, M.-J., Chou, S.-H., Chiang, M.-S., Chou, W.-H., Ko, B.-S., Huang, H.-P., Kuo, H.C., and Ho, H.-N. (2015). Characteristic expression of major histocompatibility complex and immune privilege genes in human pluripotent stem cells and their derivatives. Cell Transplant. 24, 845-864. 
Chuang, J.C., Yu, C.L., and Wang, S.R. (1990). Modulation of human lymphocyte proliferation by amino acids. Clin. Exp. Immunol. 81, 173-176.

Cobbold, S.P., Adams, E., Farquhar, C.A., Nolan, K.F., Howie, D., Lui, K.O., Fairchild, P.J., Mellor, A.L., Ron, D., and Waldmann, H. (2009). Infectious tolerance via the consumption of essential amino acids and mTOR signaling. Proc. Natl. Acad. Sci. U. S. A. 106, 12055-12060.

Colombo, P.C., Banchs, J.E., Celaj, S., Talreja, A., Lachmann, J., Malla, S., DuBois, N.B., Ashton, A.W., Latif, F., Jorde, U.P., et al. (2005). Endothelial cell activation in patients with decompensated heart failure. Circulation 111, 58-62.

Deuse, T., Wang, D., Stubbendorff, M., Itagaki, R., Grabosch, A., Greaves, L.C., Alawi, M., Grünewald, A., $\mathrm{Hu}, \mathrm{X}$. , Hua, X., et al. (2015). SCNT-derived ESCs with mismatched mitochondria trigger an immune response in allogeneic hosts. Cell Stem Cell 16, 33-38.

Dhodapkar, K.M., Feldman, D., Matthews, P., Radfar, S., Pickering, R., Turkula, S., Zebroski, H., and Dhodapkar, M.V. (2010). Natural immunity to pluripotency antigen OCT4 in humans. Proc. Natl. Acad. Sci. U. S. A. $107,8718-8723$.

Dierselhuis, M.P., Spierings, E., Drabbels, J., Hendriks, M., Alaez, C., Alberú, J., Alvarez, M.B., Burlingham, W., Campos, E., Christiaans, M., et al. (2013). Minor H antigen matches and mismatches are equally distributed among recipients with or without complications after HLA identical sibling renal transplantation. Tissue Antigens 82, 312-316.

Downey, J.M., Liu, G.S., and Thornton, J.D. (1993). Adenosine and the anti-infarct effects of preconditioning. Cardiovasc. Res. 27, 3-8.

Draper, J.S., Pigott, C., Thomson, J.A., and Andrews, P.W. (2002). Surface antigens of human embryonic stem cells: changes upon differentiation in culture. J. Anat. 200, 249-258.

Dressel, R., Schindehütte, J., Kuhlmann, T., Elsner, L., Novota, P., Baier, P.C., Schillert, A., Bickeböller, H., Herrmann, T., Trenkwalder, C., et al. (2008). The Tumorigenicity of Mouse Embryonic Stem Cells and In Vitro Differentiated Neuronal Cells Is Controlled by the Recipients' Immune Response. PLoS ONE 3, e2622.

Dressel, R., Guan, K., Nolte, J., Elsner, L., Monecke, S., Nayernia, K., Hasenfuss, G., and Engel, W. (2009). Multipotent adult germ-line stem cells, like other pluripotent stem cells, can be killed by cytotoxic $T$ lymphocytes despite low expression of major histocompatibility complex class I molecules. Biol. Direct 4 , 31.

Dressel, R., Nolte, J., Elsner, L., Novota, P., Guan, K., Streckfuss-Bomeke, K., Hasenfuss, G., Jaenisch, R., and Engel, W. (2010). Pluripotent stem cells are highly susceptible targets for syngeneic, allogeneic, and xenogeneic natural killer cells. FASEB J. 24, 2164-2177.

Drukker, M. (2006). Immunogenicity of embryonic stem cells and their progeny. Methods Enzymol. 420, 391-409.

Drukker, M., Katz, G., Urbach, A., Schuldiner, M., Markel, G., Itskovitz-Eldor, J., Reubinoff, B., Mandelboim, O., and Benvenisty, N. (2002). Characterization of the expression of MHC proteins in human embryonic stem cells. Proc. Natl. Acad. Sci. U. S. A. 99, 9864-9869. 
Dzierzak-Mietla, M., Markiewicz, M., Siekiera, U., Mizia, S., Koclega, A., Zielinska, P., Sobczyk-Kruszelnicka, M., and Kyrcz-Krzemien, S. (2012). Occurrence and Impact of Minor Histocompatibility Antigens' Disparities on Outcomes of Hematopoietic Stem Cell Transplantation from HLA-Matched Sibling Donors. Bone Marrow Res. 2012, 257086.

Eghbali, M. (1989). Cellular origin and distribution of transforming growth factor-beta in the normal rat myocardium. Cell Tissue Res. 256, 553-558.

Elsner, L., Flügge, P.F., Lozano, J., Muppala, V., Eiz-Vesper, B., Demiroglu, S.Y., Malzahn, D., Herrmann, T., Brunner, E., Bickeböller, H., et al. (2010). The endogenous danger signals HSP70 and MICA cooperate in the activation of cytotoxic effector functions of NK cells. J. Cell. Mol. Med. 14, 992-1002.

Epelman, S., Liu, P.P., and Mann, D.L. (2015). Role of innate and adaptive immune mechanisms in cardiac injury and repair. Nat. Rev. Immunol. 15, 117-129.

Esteban, M.A., Xu, J., Yang, J., Peng, M., Qin, D., Li, W., Jiang, Z., Chen, J., Deng, K., Zhong, M., et al. (2009). Generation of induced pluripotent stem cell lines from Tibetan miniature pig. J. Biol. Chem. 284, 1763417640.

Evans, M.J., and Kaufman, M.H. (1981). Establishment in culture of pluripotential cells from mouse embryos. Nature 292, 154-156.

Fallarino, F., Grohmann, U., You, S., McGrath, B.C., Cavener, D.R., Vacca, C., Orabona, C., Bianchi, R., Belladonna, M.L., Volpi, C., et al. (2006). The combined effects of tryptophan starvation and tryptophan catabolites down-regulate $\mathrm{T}$ cell receptor zeta-chain and induce a regulatory phenotype in naive $\mathrm{T}$ cells. $\mathrm{J}$. Immunol. Baltim. Md 1950 176, 6752-6761.

Fändrich, F., Lin, X., Chai, G.X., Schulze, M., Ganten, D., Bader, M., Holle, J., Huang, D.-S., Parwaresch, R., Zavazava, N., et al. (2002). Preimplantation-stage stem cells induce long-term allogeneic graft acceptance without supplementary host conditioning. Nat. Med. 8, 171-178.

Feng, L., Ke, N., Ye, Z., Guo, Y., Li, S., Li, Q., and Li, Y. (2009). Expression of NKG2D and its ligand in mouse heart allografts may have a role in acute rejection. Transplant. Proc. 41, 4332-4339.

Fernandes, E., Goold, H.D., Kissenpfennig, A., Malissen, B., Dyson, J., and Bennett, C.L. (2011). The role of direct presentation by donor dendritic cells in rejection of minor histocompatibility antigen-mismatched skin and hematopoietic cell grafts. Transplantation 91, 154-160.

Ferrara, J.L.M., Levine, J.E., Reddy, P., and Holler, E. (2009). Graft-versus-host disease. Lancet Lond. Engl. 373, 1550-1561.

Frenzel, L.P., Abdullah, Z., Kriegeskorte, A.K., Dieterich, R., Lange, N., Busch, D.H., Krönke, M., Utermöhlen, O., Hescheler, J., and Sarić, T. (2009). Role of natural-killer group 2 member D ligands and intercellular adhesion molecule 1 in natural killer cell-mediated lysis of murine embryonic stem cells and embryonic stem cell-derived cardiomyocytes. Stem Cells Dayt. Ohio 27, 307-316.

Froidevaux, S., and Loor, F. (1991). A quick procedure for identifying doubly homozygous immunodeficient scid beige mice. J. Immunol. Methods 137, 275-279. 
Fujinami, R.S. (2001). Viruses and autoimmune disease - two sides of the same coin? Trends Microbiol. 9, 377-381.

Fusaki, N., Ban, H., Nishiyama, A., Saeki, K., and Hasegawa, M. (2009). Efficient induction of transgenefree human pluripotent stem cells using a vector based on Sendai virus, an RNA virus that does not integrate into the host genome. Proc. Jpn. Acad. Ser. B Phys. Biol. Sci. 85, 348-362.

Ghosh, Z., Wilson, K.D., Wu, Y., Hu, S., Quertermous, T., and Wu, J.C. (2010). Persistent donor cell gene expression among human induced pluripotent stem cells contributes to differences with human embryonic stem cells. PloS One 5, e8975.

Goulmy, E. (1996). Human minor histocompatibility antigens. Curr. Opin. Immunol. 8, 75-81.

Goulmy, E., Termijtelen, A., Bradley, B.A., and van Rood, J.J. (1976). Alloimmunity to human H-Y. Lancet Lond. Engl. 2, 1206.

Goulmy, E., Schipper, R., Pool, J., Blokland, E., Falkenburg, J.H., Vossen, J., Gratwohl, A., Vogelsang, G.B., van Houwelingen, H.C., and van Rood, J.J. (1996). Mismatches of minor histocompatibility antigens between HLA-identical donors and recipients and the development of graft-versus-host disease after bone marrow transplantation. N. Engl. J. Med. 334, 281-285.

Grabie, N., Hsieh, D.T., Buono, C., Westrich, J.R., Allen, J.A., Pang, H., Stavrakis, G., and Lichtman, A.H. (2003a). Neutrophils sustain pathogenic CD8+T cell responses in the heart. Am. J. Pathol. 163, 2413-2420.

Grabie, N., Delfs, M.W., Westrich, J.R., Love, V.A., Stavrakis, G., Ahmad, F., Seidman, C.E., Seidman, J.G., and Lichtman, A.H. (2003b). IL-12 is required for differentiation of pathogenic CD8+ T cell effectors that cause myocarditis. J. Clin. Invest. 111, 671-680.

Gratwohl, A., Döhler, B., Stern, M., and Opelz, G. (2008). H-Y as a minor histocompatibility antigen in kidney transplantation: a retrospective cohort study. Lancet Lond. Engl. 372, 49-53.

Guan, K., Nayernia, K., Maier, L.S., Wagner, S., Dressel, R., Lee, J.H., Nolte, J., Wolf, F., Li, M., Engel, W., et al. (2006). Pluripotency of spermatogonial stem cells from adult mouse testis. Nature 440, 1199-1203.

Guha, P., Morgan, J.W., Mostoslavsky, G., Rodrigues, N.P., and Boyd, A.S. (2013). Lack of Immune Response to Differentiated Cells Derived from Syngeneic Induced Pluripotent Stem Cells. Cell Stem Cell $12,407-412$.

Hammond, G.L., Lai, Y.K., and Markert, C.L. (1984). Preliminary characterization of molecules that increase cell free translational activity of cardiac cytoplasmic RNA. Eur. Heart J. 5 Suppl F, 225-229.

Han, K.H., Ro, H., Hong, J.H., Lee, E.M., Cho, B., Yeom, H.J., Kim, M.-G., Oh, K.-H., Ahn, C., and Yang, J. (2011). Immunosuppressive mechanisms of embryonic stem cells and mesenchymal stem cells in alloimmune response. Transpl. Immunol. 25, 7-15.

Heinold, A., Opelz, G., Scherer, S., Ruhenstroth, A., Laux, G., Doehler, B., and Tran, T.H. (2008). Role of minor histocompatibility antigens in renal transplantation. Am. J. Transplant. Off. J. Am. Soc. Transplant. Am. Soc. Transpl. Surg. 8, 95-102. 
von Herrath, M.G., Fujinami, R.S., and Whitton, J.L. (2003). Microorganisms and autoimmunity: making the barren field fertile? Nat. Rev. Microbiol. 1, 151-157.

Hershberg, R.M., Cho, D.H., Youakim, A., Bradley, M.B., Lee, J.S., Framson, P.E., and Nepom, G.T. (1998). Highly polarized HLA class II antigen processing and presentation by human intestinal epithelial cells. J. Clin. Invest. 102, 792-803.

Heymans, S., Hirsch, E., Anker, S.D., Aukrust, P., Balligand, J.-L., Cohen-Tervaert, J.W., Drexler, H., Filippatos, G., Felix, S.B., Gullestad, L., et al. (2009). Inflammation as a therapeutic target in heart failure? A scientific statement from the Translational Research Committee of the Heart Failure Association of the European Society of Cardiology. Eur. J. Heart Fail. 11, 119-129.

Hogquist, K.A., Jameson, S.C., Heath, W.R., Howard, J.L., Bevan, M.J., and Carbone, F.R. (1994). T cell receptor antagonist peptides induce positive selection. Cell 76, 17-27.

Hombrink, P., Hassan, C., Kester, M.G.D., de Ru, A.H., van Bergen, C.A.M., Nijveen, H., Drijfhout, J.W., Falkenburg, J.H.F., Heemskerk, M.H.M., and van Veelen, P.A. (2013). Discovery of T cell epitopes implementing HLA-peptidomics into a reverse immunology approach. J. Immunol. Baltim. Md 1950 190, 3869-3877.

Honda, A., Hirose, M., Hatori, M., Matoba, S., Miyoshi, H., Inoue, K., and Ogura, A. (2010). Generation of induced pluripotent stem cells in rabbits: potential experimental models for human regenerative medicine. J. Biol. Chem. 285, 31362-31369.

Hu, B.-Y., Weick, J.P., Yu, J., Ma, L.-X., Zhang, X.-Q., Thomson, J.A., and Zhang, S.-C. (2010). Neural differentiation of human induced pluripotent stem cells follows developmental principles but with variable potency. Proc. Natl. Acad. Sci. U. S. A. 107, 4335-4340.

Ismahil, M.A., Hamid, T., Bansal, S.S., Patel, B., Kingery, J.R., and Prabhu, S.D. (2014). Remodeling of the mononuclear phagocyte network underlies chronic inflammation and disease progression in heart failure: critical importance of the cardiosplenic axis. Circ. Res. 114, 266-282.

Jurisicova, A., Casper, R.F., MacLusky, N.J., Mills, G.B., and Librach, C.L. (1996). HLA-G expression during preimplantation human embryo development. Proc. Natl. Acad. Sci. U. S. A. 93, 161-165.

Kaya, Z., Göser, S., Buss, S.J., Leuschner, F., Ottl, R., Li, J., Völkers, M., Zittrich, S., Pfitzer, G., Rose, N.R., et al. (2008). Identification of cardiac troponin I sequence motifs leading to heart failure by induction of myocardial inflammation and fibrosis. Circulation 118, 2063-2072.

Kaya, Z., Leib, C., and Katus, H.A. (2012). Autoantibodies in Heart Failure and Cardiac Dysfunction. Circ. Res. 110, 145-158.

Kim, J., Chang, C.K., Hayden, T., Liu, F.-C., Benjamin, J., Hamerman, J.A., Lanier, L.L., and Kang, S.-M. (2007). The activating immunoreceptor NKG2D and its ligands are involved in allograft transplant rejection. J. Immunol. Baltim. Md 1950 179, 6416-6420.

Kisseberth, W.C., Brettingen, N.T., Lohse, J.K., and Sandgren, E.P. (1999). Ubiquitous expression of marker transgenes in mice and rats. Dev. Biol. 214, 128-138. 
Koch, C.A., Geraldes, P., and Platt, J.L. (2008). Immunosuppression by embryonic stem cells. Stem Cells Dayt. Ohio 26, 89-98.

Koch, J., Steinle, A., Watzl, C., and Mandelboim, O. (2013). Activating natural cytotoxicity receptors of natural killer cells in cancer and infection. Trends Immunol. 34, 182-191.

Kofidis, T., deBruin, J.L., Tanaka, M., Zwierzchoniewska, M., Weissman, I., Fedoseyeva, E., Haverich, A., and Robbins, R.C. (2005). They are not stealthy in the heart: embryonic stem cells trigger cell infiltration, humoral and T-lymphocyte-based host immune response. Eur. J. Cardio-Thorac. Surg. Off. J. Eur. Assoc. Cardio-Thorac. Surg. 28, 461-466.

Koh, S., Tsai, S., Bischoff, S., Olby, N.J., and Piedrahita, J. (2010). Generation of Putative Induced Pluripotent Stem Cells from Adult Canine Fibroblast by Three Factor Reprogramming (Oct3/4, KIf4, and Sox2). Biol. Reprod. 83, 700.

Koller, L., Richter, B., Goliasch, G., Blum, S., Korpak, M., Zorn, G., Brekalo, M., Maurer, G., Wojta, J., Pacher, R., et al. (2013). CD4+ CD28(null) cells are an independent predictor of mortality in patients with heart failure. Atherosclerosis 230, 414-416.

Komiyama, Y., Nakae, S., Matsuki, T., Nambu, A., Ishigame, H., Kakuta, S., Sudo, K., and Iwakura, Y. (2006). IL-17 plays an important role in the development of experimental autoimmune encephalomyelitis. J. Immunol. Baltim. Md 1950 177, 566-573.

Korczyk, D., Marwick, T.H., and Kaye, G. (2012). Fast Facts: Heart Failure (Abingdon, Oxford: Health Press Limited).

Korrer, M.J., and Routes, J.M. (2014). Possible Role of Arginase-1 in Concomitant Tumor Immunity. PLoS ONE 9.

Krco, C.J., and Goldberg, E.H. (1976). H-Y male antigen: detection on eight-cell mouse embryos. Science 193, 1134-1135.

Kreisel, D., Krupnick, A.S., Gelman, A.E., Engels, F.H., Popma, S.H., Krasinskas, A.M., Balsara, K.R., Szeto, W.Y., Turka, L.A., and Rosengard, B.R. (2002). Non-hematopoietic allograft cells directly activate CD8+ T cells and trigger acute rejection: an alternative mechanism of allorecognition. Nat. Med. 8, 233-239.

Kruse, V., Hamann, C., Monecke, S., Cyganek, L., Elsner, L., Hübscher, D., Walter, L., Streckfuss-Bömeke, K., Guan, K., and Dressel, R. (2015). Human Induced Pluripotent Stem Cells Are Targets for Allogeneic and Autologous Natural Killer (NK) Cells and Killing Is Partly Mediated by the Activating NK Receptor DNAM-1. PLoS ONE 10, e0125544.

Kuzmenkin, A., Liang, H., Xu, G., Pfannkuche, K., Eichhorn, H., Fatima, A., Luo, H., Saric, T., Wernig, M., Jaenisch, R., et al. (2009). Functional characterization of cardiomyocytes derived from murine induced pluripotent stem cells in vitro. FASEB J. Off. Publ. Fed. Am. Soc. Exp. Biol. 23, 4168-4180.

Laflamme, M.A., and Murry, C.E. (2011). Heart regeneration. Nature 473, 326-335.

Laflamme, M.A., Chen, K.Y., Naumova, A.V., Muskheli, V., Fugate, J.A., Dupras, S.K., Reinecke, H., Xu, C., Hassanipour, M., Police, S., et al. (2007). Cardiomyocytes derived from human embryonic stem cells in pro-survival factors enhance function of infarcted rat hearts. Nat. Biotechnol. 25, 1015-1024. 
Laroumanie, F., Douin-Echinard, V., Pozzo, J., Lairez, O., Tortosa, F., Vinel, C., Delage, C., Calise, D., Dutaur, M., Parini, A., et al. (2014). CD4+ T Cells Promote the Transition From Hypertrophy to Heart Failure During Chronic Pressure Overload. Circulation 129, 2111-2124.

Lavine, K.J., Epelman, S., Uchida, K., Weber, K.J., Nichols, C.G., Schilling, J.D., Ornitz, D.M., Randolph, G.J., and Mann, D.L. (2014). Distinct macrophage lineages contribute to disparate patterns of cardiac recovery and remodeling in the neonatal and adult heart. Proc. Natl. Acad. Sci. U. S. A. 111, 16029-16034.

Lechler, R.I., Sykes, M., Thomson, A.W., and Turka, L.A. (2005). Organ transplantation--how much of the promise has been realized? Nat. Med. 11, 605-613.

Li, J., Hampton, T., Morgan, J.P., and Simons, M. (1997). Stretch-induced VEGF expression in the heart. J. Clin. Invest. 100, 18-24.

Li, J., Li, D., and Tan, Z. (2004a). The expression of interleukin-17, interferon-gamma, and macrophage inflammatory protein-3 alpha mRNA in patients with psoriasis vulgaris. J. Huazhong Univ. Sci. Technol. Med. Sci. Hua Zhong Ke Ji Xue Xue Bao Yi Xue Ying Wen Ban Huazhong Keji Daxue Xuebao Yixue Yingdewen Ban 24, 294-296.

Li, L., Baroja, M.L., Majumdar, A., Chadwick, K., Rouleau, A., Gallacher, L., Ferber, I., Lebkowski, J., Martin, T., Madrenas, J., et al. (2004b). Human embryonic stem cells possess immune-privileged properties. Stem Cells Dayt. Ohio 22, 448-456.

Liao, Y.-H., Xia, N., Zhou, S.-F., Tang, T.-T., Yan, X.-X., Lv, B.-J., Nie, S.-F., Wang, J., Iwakura, Y., Xiao, H., et al. (2012). Interleukin-17A contributes to myocardial ischemia/reperfusion injury by regulating cardiomyocyte apoptosis and neutrophil infiltration. J. Am. Coll. Cardiol. 59, 420-429.

Libbey, J.E., and Fujinami, R.S. (2010). Potential triggers of MS. Results Probl. Cell Differ. 51, 21-42.

Lichtman, A.H. (2013). The heart of the matter: protection of the myocardium from T cells. J. Autoimmun. 45, 90-96.

Limas, C.J., and Limas, C. (1991). Beta-adrenoceptor antibodies and genetics in dilated cardiomyopathy-an overview and review. Eur. Heart J. 12 Suppl $D, 175-177$.

Littman, D.R., and Rudensky, A.Y. (2010). Th17 and regulatory T cells in mediating and restraining inflammation. Cell 140, 845-858.

Liu, H., Zhu, F., Yong, J., Zhang, P., Hou, P., Li, H., Jiang, W., Cai, J., Liu, M., Cui, K., et al. (2008). Generation of induced pluripotent stem cells from adult rhesus monkey fibroblasts. Cell Stem Cell 3, 587-590.

Liu, H.R., Zhao, R.R., Jiao, X.Y., Wang, Y.Y., and Fu, M. (2002). Relationship of myocardial remodeling to the genesis of serum autoantibodies to cardiac beta(1)-adrenoceptors and muscarinic type 2 acetylcholine receptors in rats. J. Am. Coll. Cardiol. 39, 1866-1873.

Liu, Y., Elf, S.E., Miyata, Y., Sashida, G., Liu, Y., Huang, G., Di Giandomenico, S., Lee, J.M., Deblasio, A., Menendez, S., et al. (2009). p53 regulates hematopoietic stem cell quiescence. Cell Stem Cell 4, 37-48.

Livak, K.J., and Schmittgen, T.D. (2001). Analysis of relative gene expression data using real-time quantitative PCR and the 2(-Delta Delta C(T)) Method. Methods San Diego Calif 25, 402-408. 
López-Larrea, C. (2012). Stem cell transplantation (New York, N.Y. : Austin, Tex: Springer Science+Business Media ; Landes Bioscience).

Lv, H., Havari, E., Pinto, S., Gottumukkala, R.V.S.R.K., Cornivelli, L., Raddassi, K., Matsui, T., Rosenzweig, A., Bronson, R.T., Smith, R., et al. (2011). Impaired thymic tolerance to $\alpha$-myosin directs autoimmunity to the heart in mice and humans. J. Clin. Invest. 121, 1561-1573.

Magliocca, J.F., Held, I.K.A., and Odorico, J.S. (2006). Undifferentiated murine embryonic stem cells cannot induce portal tolerance but may possess immune privilege secondary to reduced major histocompatibility complex antigen expression. Stem Cells Dev. 15, 707-717.

Magnusson, Y., Wallukat, G., Waagstein, F., Hjalmarson, A., and Hoebeke, J. (1994). Autoimmunity in idiopathic dilated cardiomyopathy. Characterization of antibodies against the beta 1-adrenoceptor with positive chronotropic effect. Circulation 89, 2760-2767.

Mann, D.L. (2003). Stress-Activated Cytokines and The Heart: From Adaptation to Maladaptation. Annu. Rev. Physiol. 65, 81-101.

Mann, D.L. (2015). Innate Immunity and the Failing Heart The Cytokine Hypothesis Revisited. Circ. Res. 116, 1254-1268.

Marchetto, M.C.N., Yeo, G.W., Kainohana, O., Marsala, M., Gage, F.H., and Muotri, A.R. (2009). Transcriptional signature and memory retention of human-induced pluripotent stem cells. PloS One 4, e7076.

Markiewicz, M., Siekiera, U., Karolczyk, A., Szymszal, J., Helbig, G., Wojnar, J., Dzierzak-Mietla, M., and Kyrcz-Krzemien, S. (2009). Immunogenic disparities of 11 minor histocompatibility antigens (mHAs) in HLA-matched unrelated allogeneic hematopoietic SCT. Bone Marrow Transplant. 43, 293-300.

Martin, G.R. (1981). Isolation of a pluripotent cell line from early mouse embryos cultured in medium conditioned by teratocarcinoma stem cells. Proc. Natl. Acad. Sci. U. S. A. 78, 7634-7638.

Maynard, K.M., Arvindam, U., Cross, M., and Firpo, M.T. (2014). Potentially immunogenic proteins expressed similarly in human embryonic stem cells and induced pluripotent stem cells. Exp. Biol. Med. Maywood NJ 239, 484-488.

McMurray, J.J.V. (2010). Clinical practice. Systolic heart failure. N. Engl. J. Med. 362, 228-238.

Metz, R., Duhadaway, J.B., Kamasani, U., Laury-Kleintop, L., Muller, A.J., and Prendergast, G.C. (2007). Novel tryptophan catabolic enzyme IDO2 is the preferred biochemical target of the antitumor indoleamine 2,3-dioxygenase inhibitory compound D-1-methyl-tryptophan. Cancer Res. 67, 7082-7087.

Mölne, J., Björquist, P., Andersson, K., Diswall, M., Jeppsson, A., Strokan, V., Rydberg, L., and Breimer, M.E. (2008). Blood group $A B O$ antigen expression in human embryonic stem cells and in differentiated hepatocyte- and cardiomyocyte-like cells. Transplantation 86, 1407-1413.

Mombaerts, P., lacomini, J., Johnson, R.S., Herrup, K., Tonegawa, S., and Papaioannou, V.E. (1992). RAG1-deficient mice have no mature B and T lymphocytes. Cell 68, 869-877.

Monecke, S. (2013). Immunogenicity of pluripotent stem cells and their differentiation products. 
Morelli, A.E., and Thomson, A.W. (2003). Dendritic cells: regulators of alloimmunity and opportunities for tolerance induction. Immunol. Rev. 196, 125-146.

Mosterd, A., and Hoes, A.W. (2007). Clinical epidemiology of heart failure. Heart Br. Card. Soc. 93, 11371146.

Mozaffarian, D., Benjamin, E.J., Go, A.S., Arnett, D.K., Blaha, M.J., Cushman, M., Ferranti, S. de, Després, J.-P., Fullerton, H.J., Howard, V.J., et al. (2014). Heart Disease and Stroke Statistics-2015 Update A Report From the American Heart Association. Circulation CIR.0000000000000152.

Munn, D.H., and Mellor, A.L. (2007). Indoleamine 2,3-dioxygenase and tumor-induced tolerance. J. Clin. Invest. 117, 1147-1154.

Munn, D.H., Zhou, M., Attwood, J.T., Bondarev, I., Conway, S.J., Marshall, B., Brown, C., and Mellor, A.L. (1998). Prevention of allogeneic fetal rejection by tryptophan catabolism. Science 281, 1191-1193.

Mutis, T. (2015). HY antibodies as biomarkers for chronic GVHD. Blood 125, 3046-3047.

Nakasone, H., Tian, L., Sahaf, B., Kawase, T., Schoenrock, K., Perloff, S., Ryan, C.E., Paul, J., Popli, R., Wu, F., et al. (2015). Allogeneic HY antibodies detected 3 months after female-to-male HCT predict chronic GVHD and nonrelapse mortality in humans. Blood 125, 3193-3201.

Narazaki, G., Uosaki, H., Teranishi, M., Okita, K., Kim, B., Matsuoka, S., Yamanaka, S., and Yamashita, J.K. (2008). Directed and systematic differentiation of cardiovascular cells from mouse induced pluripotent stem cells. Circulation 118, 498-506.

Nevers, T., Salvador, A.M., Grodecki-Pena, A., Knapp, A., Velázquez, F., Aronovitz, M., Kapur, N.K., Karas, R.H., Blanton, R.M., and Alcaide, P. (2015). Left Ventricular T-Cell Recruitment Contributes to the Pathogenesis of Heart Failure. Circ. Heart Fail. 8, 776-787.

Nussbaum, J., Minami, E., Laflamme, M.A., Virag, J.A.I., Ware, C.B., Masino, A., Muskheli, V., Pabon, L., Reinecke, H., and Murry, C.E. (2007). Transplantation of undifferentiated murine embryonic stem cells in the heart: teratoma formation and immune response. FASEB J. Off. Publ. Fed. Am. Soc. Exp. Biol. 21, 13451357.

Ogasawara, K., and Lanier, L.L. (2005). NKG2D in NK and T cell-mediated immunity. J. Clin. Immunol. 25, 534-540.

Ogasawara, K., Hamerman, J.A., Ehrlich, L.R., Bour-Jordan, H., Santamaria, P., Bluestone, J.A., and Lanier, L.L. (2004). NKG2D Blockade Prevents Autoimmune Diabetes in NOD Mice. Immunity 20, 757-767.

Ogasawara, K., Benjamin, J., Takaki, R., Phillips, J.H., and Lanier, L.L. (2005). Function of NKG2D in natural killer cell-mediated rejection of mouse bone marrow grafts. Nat. Immunol. 6, 938-945.

Okita, K., Nakagawa, M., Hyenjong, H., Ichisaka, T., and Yamanaka, S. (2008). Generation of mouse induced pluripotent stem cells without viral vectors. Science 322, 949-953.

Opelz, G., and Collaborative Transplant Study (2005). Non-HLA transplantation immunity revealed by lymphocytotoxic antibodies. Lancet Lond. Engl. 365, 1570-1576. 
Ozaki, Y., Edelstein, M.P., and Duch, D.S. (1988). Induction of indoleamine 2,3-dioxygenase: a mechanism of the antitumor activity of interferon gamma. Proc. Natl. Acad. Sci. U. S. A. 85, 1242-1246.

Pan, K., Wang, H., Chen, M., Zhang, H., Weng, D., Zhou, J., Huang, W., Li, J., Song, H., and Xia, J. (2008). Expression and prognosis role of indoleamine 2,3-dioxygenase in hepatocellular carcinoma. J. Cancer Res. Clin. Oncol. 134, 1247-1253.

Park, I.-H., Arora, N., Huo, H., Maherali, N., Ahfeldt, T., Shimamura, A., Lensch, M.W., Cowan, C., Hochedlinger, K., and Daley, G.Q. (2008). Disease-specific induced pluripotent stem cells. Cell 134, 877886.

Patil, M.D., Bhaumik, J., Babykutty, S., Banerjee, U.C., and Fukumura, D. (2016). Arginine dependence of tumor cells: targeting a chink in cancer's armor. Oncogene.

Patten, R.D., and Hall-Porter, M.R. (2009). Small Animal Models of Heart Failure Development of Novel Therapies, Past and Present. Circ. Heart Fail. 2, 138-144.

Pearl, J.I., Lee, A.S., Leveson-Gower, D.B., Sun, N., Ghosh, Z., Lan, F., Ransohoff, J., Negrin, R.S., Davis, M.M., and Wu, J.C. (2011). Short-Term Immunosuppression Promotes Engraftment of Embryonic and Induced Pluripotent Stem Cells. Cell Stem Cell 8, 309-317.

Pegram, H.J., Andrews, D.M., Smyth, M.J., Darcy, P.K., and Kershaw, M.H. (2011). Activating and inhibitory receptors of natural killer cells. Immunol. Cell Biol. 89, 216-224.

Petrov, A., Hamann, C., Monecke, S., Dressel, R. (unpublished data). MD thesis.

Pick, M., Stelzer, Y., Bar-Nur, O., Mayshar, Y., Eden, A., and Benvenisty, N. (2009). Clone- and gene-specific aberrations of parental imprinting in human induced pluripotent stem cells. Stem Cells Dayt. Ohio 27, 2686-2690.

Pla, M., and Mahouy, G. (1991). The SCID mouse. Nouv. Rev. Fr. Hématologie 33, 489-491.

Platten, M., Wick, W., and Van den Eynde, B.J. (2012). Tryptophan catabolism in cancer: beyond IDO and tryptophan depletion. Cancer Res. 72, 5435-5440.

Plumas, J., Chaperot, L., Richard, M.-J., Molens, J.-P., Bensa, J.-C., and Favrot, M.-C. (2005). Mesenchymal stem cells induce apoptosis of activated T cells. Leukemia 19, 1597-1604.

Porcheray, F., Miklos, D.B., Floyd, B.H., Sarantopoulos, S., Bellucci, R., Soiffer, R.J., Antin, J.H., Alyea, E.P., Ritz, J., and Zorn, E. (2011). Combined CD4 T-cell and antibody response to human minor histocompatibility antigen DBY after allogeneic stem-cell transplantation. Transplantation 92, 359-365.

Purcell, N.H., Tang, G., Yu, C., Mercurio, F., DiDonato, J.A., and Lin, A. (2001). Activation of NF-kappa B is required for hypertrophic growth of primary rat neonatal ventricular cardiomyocytes. Proc. Natl. Acad. Sci. U. S. A. 98, 6668-6673.

Qian, L., Huang, Y., Spencer, C.I., Foley, A., Vedantham, V., Liu, L., Conway, S.J., Fu, J., and Srivastava, D. (2012). In vivo reprogramming of murine cardiac fibroblasts into induced cardiomyocytes. Nature 485, 593-598. 
Rao, M., and Condic, M.L. (2008). Alternative sources of pluripotent stem cells: scientific solutions to an ethical dilemma. Stem Cells Dev. 17, 1-10.

Raulet, D.H. (2006). Missing self recognition and self tolerance of natural killer (NK) cells. Semin. Immunol. $18,145-150$.

Robertson, N.J., Brook, F.A., Gardner, R.L., Cobbold, S.P., Waldmann, H., and Fairchild, P.J. (2007). Embryonic stem cell-derived tissues are immunogenic but their inherent immune privilege promotes the induction of tolerance. Proc. Natl. Acad. Sci. U. S. A. 104, 20920-20925.

Rockman, H.A., Ross, R.S., Harris, A.N., Knowlton, K.U., Steinhelper, M.E., Field, L.J., Ross, J., and Chien, K.R. (1991). Segregation of atrial-specific and inducible expression of an atrial natriuretic factor transgene in an in vivo murine model of cardiac hypertrophy. Proc. Natl. Acad. Sci. U. S. A. $88,8277-8281$.

Rohani, L., Fabian, C., Holland, H., Naaldijk, Y., Dressel, R., Löffler-Wirth, H., Binder, H., Arnold, A., and Stolzing, A. (2016). Generation of human induced pluripotent stem cells using non-synthetic mRNA. Stem Cell Res. 16, 662-672.

Röhrborn, C., Hamann, C., Monecke, S., Dressel, R., and Elsner, L. (unpublished data).MD Thesis.

Rong, X.-X., Wei, F., Lin, X.-L., Qin, Y.-J., Chen, L., Wang, H.-Y., Shen, H.-F., Jia, L.-T., Xie, R.-Y., Lin, T.-Y., et al. (2016). Recognition and killing of cancer stem-like cell population in hepatocellular carcinoma cells by cytokine-induced killer cells via NKG2d-ligands recognition. Oncoimmunology 5, e1086060.

Roopenian, D., Choi, E.Y., and Brown, A. (2002). The immunogenomics of minor histocompatibility antigens. Immunol. Rev. 190, 86-94.

Rosenberg, A.S., and Singer, A. (1992). Cellular basis of skin allograft rejection: an in vivo model of immune-mediated tissue destruction. Annu. Rev. Immunol. 10, 333-358.

Sadoshima, J., Xu, Y., Slayter, H.S., and Izumo, S. (1993). Autocrine release of angiotensin II mediates stretch-induced hypertrophy of cardiac myocytes in vitro. Cell 75, 977-984.

Salih, H.R., Antropius, H., Gieseke, F., Lutz, S.Z., Kanz, L., Rammensee, H.-G., and Steinle, A. (2003). Functional expression and release of ligands for the activating immunoreceptor NKG2D in leukemia. Blood 102, 1389-1396.

Salvador, A.M., Nevers, T., Velázquez, F., Aronovitz, M., Wang, B., Abadía Molina, A., Jaffe, I.Z., Karas, R.H., Blanton, R.M., and Alcaide, P. (2016). Intercellular Adhesion Molecule 1 Regulates Left Ventricular Leukocyte Infiltration, Cardiac Remodeling, and Function in Pressure Overload-Induced Heart Failure. J. Am. Heart Assoc. 4, e003126.

Santos, N., Rodríguez-Romanos, R., Nieto, J.B., Buño, I., Vallejo, C., Jiménez-Velasco, A., Brunet, S., Buces, E., López-Jiménez, J., González, M., et al. (2016). UGT2B17 minor histocompatibility mismatch and clinical outcome after HLA-identical sibling donor stem cell transplantation. Bone Marrow Transplant. 51, 79-82.

Sasse, A., Hamann, C., Monecke, S., Elsner, L., and Dressel, R. (unpublished data). MD Thesis. 
Schenke-Layland, K., Rhodes, K.E., Angelis, E., Butylkova, Y., Heydarkhan-Hagvall, S., Gekas, C., Zhang, R., Goldhaber, J.I., Mikkola, H.K., Plath, K., et al. (2008). Reprogrammed mouse fibroblasts differentiate into cells of the cardiovascular and hematopoietic lineages. Stem Cells Dayt. Ohio 26, 1537-1546.

Senju, S., Suemori, H., Zembutsu, H., Uemura, Y., Hirata, S., Fukuma, D., Matsuyoshi, H., Shimomura, M., Haruta, M., Fukushima, S., et al. (2007). Genetically manipulated human embryonic stem cell-derived dendritic cells with immune regulatory function. Stem Cells Dayt. Ohio 25, 2720-2729.

Shiba, Y., Fernandes, S., Zhu, W.-Z., Filice, D., Muskheli, V., Kim, J., Palpant, N.J., Gantz, J., Moyes, K.W., Reinecke, H., et al. (2012). Human ES-cell-derived cardiomyocytes electrically couple and suppress arrhythmias in injured hearts. Nature 489, 322-325.

Shinkai, Y., Rathbun, OGary, Lam, K.-P., Oltz, E.M., Stewart, V., Mendelsohn, M., Charron, J., Datta, M., Young, F., Stall, A.M., et al. (1992). RAG-2-deficient mice lack mature lymphocytes owing to inability to initiate V(D)J rearrangement. Cell 68, 855-867.

Singla, D.K., Hacker, T.A., Ma, L., Douglas, P.S., Sullivan, R., Lyons, G.E., and Kamp, T.J. (2006). Transplantation of embryonic stem cells into the infarcted mouse heart: formation of multiple cell types. J. Mol. Cell. Cardiol. 40, 195-200.

Slukvin, I.I., Vodyanik, M.A., Thomson, J.A., Gumenyuk, M.E., and Choi, K.-D. (2006). Directed differentiation of human embryonic stem cells into functional dendritic cells through the myeloid pathway. J. Immunol. Baltim. Md 1950 176, 2924-2932.

Soldner, F., Hockemeyer, D., Beard, C., Gao, Q., Bell, G.W., Cook, E.G., Hargus, G., Blak, A., Cooper, O., Mitalipova, M., et al. (2009). Parkinson's disease patient-derived induced pluripotent stem cells free of viral reprogramming factors. Cell 136, 964-977.

Song, K., Nam, Y.-J., Luo, X., Qi, X., Tan, W., Huang, G.N., Acharya, A., Smith, C.L., Tallquist, M.D., Neilson, E.G., et al. (2012). Heart repair by reprogramming non-myocytes with cardiac transcription factors. Nature 485, 599-604.

Spierings, E., Vermeulen, C.J., Vogt, M.H., Doerner, L.E.E., Falkenburg, J.H.F., Mutis, T., and Goulmy, E. (2003). Identification of HLA class II-restricted H-Y-specific T-helper epitope evoking CD4+ T-helper cells in H-Y-mismatched transplantation. Lancet Lond. Engl. 362, 610-615.

Su, J., Chen, X., Huang, Y., Li, W., Li, J., Cao, K., Cao, G., Zhang, L., Li, F., Roberts, A.I., et al. (2014). Phylogenetic distinction of iNOS and IDO function in mesenchymal stem cell-mediated immunosuppression in mammalian species. Cell Death Differ. 21, 388-396.

Suárez-Alvarez, B., Rodriguez, R.M., Calvanese, V., Blanco-Gelaz, M.A., Suhr, S.T., Ortega, F., Otero, J., Cibelli, J.B., Moore, H., Fraga, M.F., et al. (2010). Epigenetic mechanisms regulate MHC and antigen processing molecules in human embryonic and induced pluripotent stem cells. PloS One 5, e10192.

Suh, W.-K., Gajewska, B.U., Okada, H., Gronski, M.A., Bertram, E.M., Dawicki, W., Duncan, G.S., Bukczynski, J., Plyte, S., Elia, A., et al. (2003). The B7 family member B7-H3 preferentially down-regulates T helper type 1-mediated immune responses. Nat. Immunol. 4, 899-906. 
Swijnenburg, R.-J., Tanaka, M., Vogel, H., Baker, J., Kofidis, T., Gunawan, F., Lebl, D.R., Caffarelli, A.D., de Bruin, J.L., Fedoseyeva, E.V., et al. (2005). Embryonic stem cell immunogenicity increases upon differentiation after transplantation into ischemic myocardium. Circulation 112, I166-I172.

Takahashi, K., and Yamanaka, S. (2006). Induction of pluripotent stem cells from mouse embryonic and adult fibroblast cultures by defined factors. Cell 126, 663-676.

Takahashi, K., Tanabe, K., Ohnuki, M., Narita, M., Ichisaka, T., Tomoda, K., and Yamanaka, S. (2007). Induction of pluripotent stem cells from adult human fibroblasts by defined factors. Cell 131, 861-872.

Tan, J.C., Wadia, P.P., Coram, M., Grumet, F.C., Kambham, N., Miller, K., Pereira, S., Vayntrub, T., and Miklos, D.B. (2008). H-Y antibody development associates with acute rejection in female patients with male kidney transplants. Transplantation $86,75-81$.

Thaik, C.M., Calderone, A., Takahashi, N., and Colucci, W.S. (1995). Interleukin-1 beta modulates the growth and phenotype of neonatal rat cardiac myocytes. J. Clin. Invest. 96, 1093.

Thaker, A.I., Rao, M.S., Bishnupuri, K.S., Kerr, T.A., Foster, L., Marinshaw, J.M., Newberry, R.D., Stenson, W.F., and Ciorba, M.A. (2013). IDO1 metabolites activate $\beta$-catenin signaling to promote cancer cell proliferation and colon tumorigenesis in mice. Gastroenterology 145, 416-425.e1-e4.

Thomson, J.A., Itskovitz-Eldor, J., Shapiro, S.S., Waknitz, M.A., Swiergiel, J.J., Marshall, V.S., and Jones, J.M. (1998). Embryonic stem cell lines derived from human blastocysts. Science 282, 1145-1147.

Tian, L., Catt, J.W., O'Neill, C., and King, N.J. (1997). Expression of immunoglobulin superfamily cell adhesion molecules on murine embryonic stem cells. Biol. Reprod. 57, 561-568.

Tian, X., Woll, P.S., Morris, J.K., Linehan, J.L., and Kaufman, D.S. (2006). Hematopoietic engraftment of human embryonic stem cell-derived cells is regulated by recipient innate immunity. Stem Cells Dayt. Ohio $24,1370-1380$.

Toischer, K., Rokita, A.G., Unsold, B., Zhu, W., Kararigas, G., Sossalla, S., Reuter, S.P., Becker, A., Teucher, N., Seidler, T., et al. (2010). Differential Cardiac Remodeling in Preload Versus Afterload. Circulation 122, 993-1003.

Topkara, V.K., Evans, S., Zhang, W., Epelman, S., Staloch, L., Barger, P.M., and Mann, D.L. (2011). Therapeutic targeting of innate immunity in the failing heart. J. Mol. Cell. Cardiol. 51, 594-599.

Tousoulis, D., Charakida, M., and Stefanadis, C. (2005). Inflammation and endothelial dysfunction as therapeutic targets in patients with heart failure. Int. J. Cardiol. 100, 347-353.

Tseng, L.H., Lin, M.T., Hansen, J.A., Gooley, T., Pei, J., Smith, A.G., Martin, E.G., Petersdorf, E.W., and Martin, P.J. (1999). Correlation between disparity for the minor histocompatibility antigen HA-1 and the development of acute graft-versus-host disease after allogeneic marrow transplantation. Blood 94, 29112914.

Uccelli, A., Moretta, L., and Pistoia, V. (2008). Mesenchymal stem cells in health and disease. Nat. Rev. Immunol. 8, 726-736. 
Vandesompele, J., De Preter, K., Pattyn, F., Poppe, B., Van Roy, N., De Paepe, A., and Speleman, F. (2002). Accurate normalization of real-time quantitative RT-PCR data by geometric averaging of multiple internal control genes. Genome Biol. 3, RESEARCH0034.

Vivier, E., Ugolini, S., Blaise, D., Chabannon, C., and Brossay, L. (2012). Targeting natural killer cells and natural killer T cells in cancer. Nat. Rev. Immunol. 12, 239-252.

Voogt, P.J., Goulmy, E., Fibbe, W.E., Veenhof, W.F., Brand, A., and Falkenburg, J.H. (1988). Minor histocompatibility antigen $\mathrm{H}-\mathrm{Y}$ is expressed on human hematopoietic progenitor cells. J. Clin. Invest. 82, 906-912.

Wallny, H.J., and Rammensee, H.G. (1990). Identification of classical minor histocompatibility antigen as cell-derived peptide. Nature 343, 275-278.

Warren, E.H., Greenberg, P.D., and Riddell, S.R. (1998). Cytotoxic T-lymphocyte-defined human minor histocompatibility antigens with a restricted tissue distribution. Blood 91, 2197-2207.

Wei, L. (2011). Immunological aspect of cardiac remodeling: T lymphocyte subsets in inflammationmediated cardiac fibrosis. Exp. Mol. Pathol. 90, 74-78.

Wei, F., Rong, X.-X., Xie, R.-Y., Jia, L.-T., Wang, H.-Y., Qin, Y.-J., Chen, L., Shen, H.-F., Lin, X.-L., Yang, J., et al. (2015). Cytokine-induced killer cells efficiently kill stem-like cancer cells of nasopharyngeal carcinoma via the NKG2D-ligands recognition. Oncotarget 6, 35023-35039.

Weiner, H.L., and Swain, J.L. (1989). Acidic fibroblast growth factor mRNA is expressed by cardiac myocytes in culture and the protein is localized to the extracellular matrix. Proc. Natl. Acad. Sci. U. S. A. $86,2683-2687$.

Wernig, M., Zhao, J.-P., Pruszak, J., Hedlund, E., Fu, D., Soldner, F., Broccoli, V., Constantine-Paton, M., Isacson, O., and Jaenisch, R. (2008). Neurons derived from reprogrammed fibroblasts functionally integrate into the fetal brain and improve symptoms of rats with Parkinson's disease. Proc. Natl. Acad. Sci. U. S. A. $105,5856-5861$.

Wu, D.C., Boyd, A.S., and Wood, K.J. (2008). Embryonic stem cells and their differentiated derivatives have a fragile immune privilege but still represent novel targets of immune attack. Stem Cells Dayt. Ohio 26, 1939-1950.

Xue, T., Cho, H.C., Akar, F.G., Tsang, S.-Y., Jones, S.P., Marbán, E., Tomaselli, G.F., and Li, R.A. (2005). Functional integration of electrically active cardiac derivatives from genetically engineered human embryonic stem cells with quiescent recipient ventricular cardiomyocytes: insights into the development of cell-based pacemakers. Circulation 111, 11-20.

Yachimovich-Cohen, N., Even-Ram, S., Shufaro, Y., Rachmilewitz, J., and Reubinoff, B. (2010). Human Embryonic Stem Cells Suppress T Cell Responses via Arginase I-Dependent Mechanism. J. Immunol. 184, 1300-1308.

Yakubov, E., Rechavi, G., Rozenblatt, S., and Givol, D. (2010). Reprogramming of human fibroblasts to pluripotent stem cells using mRNA of four transcription factors. Biochem. Biophys. Res. Commun. 394, 189-193. 
Yancy, C.W., Jessup, M., Bozkurt, B., Butler, J., Casey, D.E., Drazner, M.H., Fonarow, G.C., Geraci, S.A., Horwich, T., Januzzi, J.L., et al. (2013). 2013 ACCF/AHA Guideline for the Management of Heart Failure: Executive SummaryA Report of the American College of Cardiology Foundation/American Heart Association Task Force on Practice Guidelines. J. Am. Coll. Cardiol. 62, 1495-1539.

Yen, D., Cheung, J., Scheerens, H., Poulet, F., McClanahan, T., McKenzie, B., Kleinschek, M.A., Owyang, A., Mattson, J., Blumenschein, W., et al. (2006). IL-23 is essential for T cell-mediated colitis and promotes inflammation via IL-17 and IL-6. J. Clin. Invest. 116, 1310-1316.

Yin, F.C., Spurgeon, H.A., Rakusan, K., Weisfeldt, M.L., and Lakatta, E.G. (1982). Use of tibial length to quantify cardiac hypertrophy: application in the aging rat. Am. J. Physiol. 243, H941-H947.

Yndestad, A., Holm, A.M., Müller, F., Simonsen, S., Frøland, S.S., Gullestad, L., and Aukrust, P. (2003). Enhanced expression of inflammatory cytokines and activation markers in T-cells from patients with chronic heart failure. Cardiovasc. Res. 60, 141-146.

Yndestad, A., Kristian Damås, J., Øie, E., Ueland, T., Gullestad, L., and Aukrust, P. (2006). Systemic inflammation in heart failure - The whys and wherefores. Heart Fail. Rev. 11, 83-92.

Yu, J., Vodyanik, M.A., Smuga-Otto, K., Antosiewicz-Bourget, J., Frane, J.L., Tian, S., Nie, J., Jonsdottir, G.A., Ruotti, V., Stewart, R., et al. (2007). Induced pluripotent stem cell lines derived from human somatic cells. Science 318, 1917-1920.

Yusa, K., Rad, R., Takeda, J., and Bradley, A. (2009). Generation of transgene-free induced pluripotent mouse stem cells by the piggyBac transposon. Nat. Methods 6, 363-369.

Zafirova, B., Mandarić, S., Antulov, R., Krmpotić, A., Jonsson, H., Yokoyama, W.M., Jonjić, S., and Polić, B. (2009). Altered NK Cell Development and Enhanced NK Cell-Mediated Resistance to Mouse Cytomegalovirus in NKG2D-Deficient Mice. Immunity 31, 270-282.

Zavazava, N. (2013). Embryonic Stem Cell Immunobiology (Totowa, NJ: Humana Press).

Zhan, X., Dravid, G., Ye, Z., Hammond, H., Shamblott, M., Gearhart, J., and Cheng, L. (2004). Functional antigen-presenting leucocytes derived from human embryonic stem cells in vitro. Lancet Lond. Engl. 364, 163-171.

Zhang, X., Rao, A., Sette, P., Deibert, C., Pomerantz, A., Kim, W.J., Kohanbash, G., Chang, Y., Park, Y., Engh, J., et al. (2016). IDH mutant gliomas escape natural killer cell immune surveillance by downregulation of NKG2D ligand expression. Neuro-Oncol.

Zhao, L., Cheng, G., Jin, R., Afzal, M.R., Samanta, A., Xuan, Y.-T., Girgis, M., Elias, H.K., Zhu, Y., Davani, A., et al. (2016). Deletion of Interleukin-6 Attenuates Pressure Overload-Induced Left Ventricular Hypertrophy and Dysfunction. Circ. Res.

Zhao, T., Zhang, Z.-N., Rong, Z., and Xu, Y. (2011). Immunogenicity of induced pluripotent stem cells. Nature 474, 212-215. 


\section{Curriculum vitae}

\section{Carina Hamann}

Birthday: 20.07.1988

Born in Hildesheim, Germany

\section{Address and Contact}

Department of Cellular and Molecular Immunology

Humboldtallee 34

37073 Göttingen

Labor: 0551/3913587

Email: carina.hamann@stud.uni-goettingen.de

\section{Education}

$02 / 2013-06 / 2016$

Ph.D. thesis at the Georg-August University Göttingen at the Department of Cellular and Molecular Immunology. Topic: Investigation of immune responses contributing to the pathogenesis of load-induced heart failure and the rejection of stem cell grafts

10/2010 - 11/2012 Master of Science in Developmental-, Neural- and Behavioral Biology at the Georg-August University Göttingen. Advanced modules: Molecular Neurobiology, Molecular Immunology. Thesis: Expression profiling of genes involved in antigen presentation in human pluripotent stem cells (Prof. Dr. Ralf Dressel, Institute of Cellular and Molecular Immunology Göttingen)

10/2007-10/2010 Bachelor of Science in Biology at the Georg-August University Göttingen. Major subject: Neurobiology. Thesis: Genetic analysis of the functional interaction of proteins in the PSC of hippocampal neuron cultures (Dr. Oliver Schlüter, European Neuroscience Institute Göttingen)

$7 / 2007$ Abitur at Jacobson Gymnasium Seesen, Germany. Focus subjects: Biology and Mathematics 


\section{Publications}

*Shared $1^{\text {st }}$ authorship:

*Kruse, V., *Hamann, C., Monecke, S., Cyganek, L., Elsner, L., Hübscher, D., Walter, L., Streckfuss-Bömeke, K., Guan, K., and Dressel, R. (2015). Human Induced Pluripotent Stem Cells Are Targets for Allogeneic and Autologous Natural Killer (NK) Cells and Killing Is Partly Mediated by the Activating NK Receptor DNAM-1. PLOS ONE 10, e0125544.

\section{Congress}

Hamann C, Kruse V, Monecke S, Cyganek L, Elsner L, Hübscher D, Walter L, Streckfuss-Bömeke K, Guan K, Dressel R (2015) Human induced pluripotent stem cells are targets for allogeneic and autologous natural killer (NK) cells and killing is partly mediated by the activating NK receptor DNAM-1

In: Abstracts of the 4th European Congress of Immunology, Vienna, 06.09.15 - 09.09.15, p. 232, oral presentation

Hamann C, Monecke S, Jonjic J, Polic B, Dressel R (2015) Killing of murine pluripotent stem cells largely depends on the activating NK receptor NKG2D.

In Abstracts of the Natural Killer Cell Symposium NK 2015, Göttingen. 07.10.2015-09.10.2015, p. 22, oral presentation 